\title{
Comparison Isomorphisms for Smooth Formal Schemes
}

\author{
Fabrizio Andreatta \\ Adrian Iovita
}

November 12, 2018

\section{Contents}

1 Introduction 2

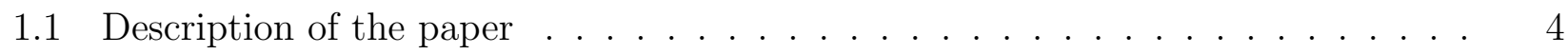

1.2 Notations . . . . . . . . . . . . . . . . . . . .

2 Fontaine sheaves 9

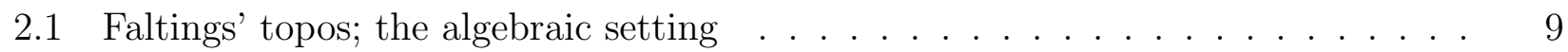

2.2 Faltings' topos; the formal setting . . . . . . . . . . . . . . . 14

2.3 Continuous functors. Localization functors . . . . . . . . . . . 15

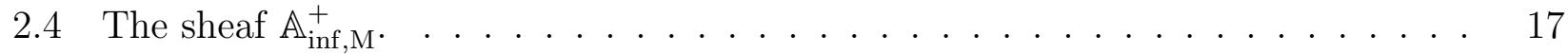

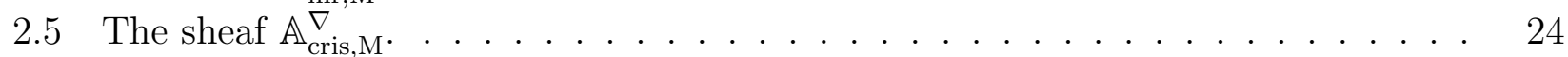

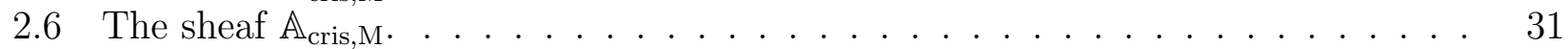

2.7 Further properties of $\mathbb{A}_{\text {cris }, \mathrm{M}}^{\nabla}$ and $\mathbb{A}_{\text {cris, } \mathrm{M}} \ldots \ldots \ldots \ldots \ldots \ldots \ldots \ldots \ldots \ldots \ldots$

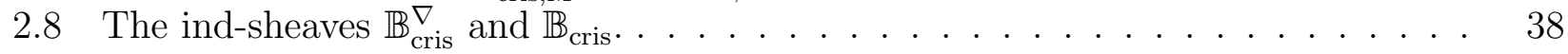

2.9 The fundamental exact sequence . . . . . . . . . . . . . . . . . 40

3 The crystalline comparison isomorphism.

3.1 Crystalline étale sheaves. . . . . . . . . . . . . . . . . 42

3.2 The functors $\mathbb{D}_{\text {cris }}^{\text {ar }}$ and $\mathbb{V}_{\text {cris }}^{\text {ar }}$ on crystalline sheaves. . . . . . . . . 50

3.3 The cohomology of crystalline sheaves. . . . . . . . . . . . . 57

3.4 The comparison isomorphism in the proper case . . . . . . . . . . 65 


\section{Introduction}

Let $p>0$ denote a prime integer and $K$ a complete discrete valuation field of characteristic 0 and perfect residue field $k$ of characteristic $p$. This article proposes a new point of view on the problem of comparison isomorphisms for algebraic varieties over $K$ which allows the extension of the results to smooth $p$-adic formal schemes over the ring of integers of $K$.

More precisely, we suppose throughout the introduction that $K$ is absolutely unramified i. e., that the prime $p$ is a uniformizer of $K$, denote by $\bar{K}$ a fixed algebraic closure of it with ring of integers $\mathcal{O}_{\bar{K}}$ and by $A_{\text {cris }}$ and $B_{\text {cris }}$ the period rings defined by J.-M. Fontaine in [Fo] (see section $\$ 1.2$ for a review of the construction). We set $G_{K}:=\operatorname{Gal}(\bar{K} / K)$.

The "comparison isomorphism problem" was first alluded to by Grothendieck as the existence of a mysterious functor associating, for a given algebraic variety over $K$, the étale cohomology groups of the variety over $\bar{K}$ to the respective de Rham cohomology groups. This was precisely formulated by J.-M. Fontaine in $[\mathrm{Fo}$ ] as the crystalline comparison conjecture. Let $X$ be a smooth proper scheme over $\mathcal{O}_{K}$. If $R$ is an $\mathcal{O}_{K}$-algebra we denote by $X_{R}$ the base change $X_{R}=X \times_{\operatorname{Spec}\left(\mathcal{O}_{K}\right)} \operatorname{Spec}(R)$. In particular we'll denote by $\bar{X}$ the special fiber $X_{k}$ of $X$.

Conjecture $1.1([\mathrm{Fo}])$. In the notations above for every $i \geq 0$ there is a canonical and functorial isomorphism commuting with all the additional structures (namely filtrations, $G_{K}-$ actions and Frobenii)

$$
\mathrm{H}^{i}\left(X \frac{\mathrm{et}}{K}, \mathbb{Q}_{p}\right) \otimes_{\mathbb{Q}_{p}} B_{\text {cris }} \cong \mathrm{H}_{\text {cris }}^{i}\left(\bar{X} / \mathcal{O}_{K}\right) \otimes_{\mathcal{O}_{K}} B_{\text {cris }}
$$

The first case of the conjecture was proved by Fontaine himself in [Fo] for abelian schemes. There followed other proofs of the conjecture for abelian varieties and curves over $K$ with good or semi-stable reduction in preprints of Fontaine-Messing (unpublished), [C], [CC].

The first general result was proved by Bloch and Kato in BK for proper and smooth schemes with ordinary reduction. The next breakthrough was due to Fontaine and Messing in [FM]. They noticed that the ring $A_{\text {cris }}$ has a geometric interpretation as global sections of a certain sheaf on the crystalline site of $\bar{X}$ and thus the syntomic cohomology on $X$ calculates the right hand side of the isomorphism in the conjecture 1.1. The fact that the syntomic cohomology can be related to the left hand side (i. e., to étale cohomology of $X_{\bar{K}}$ ) is more complicated and the conjecture was proved in [FM] under the assumption that $1+2 \operatorname{dim}\left(X_{K}\right)<p$.

G. Faltings fully proved the conjecture in [F2], in fact he proved more: one can drop the assumption that $K$ is absolutely unramified and he allowed certain non-trivial coefficients, more precisely $\mathbb{Q}_{p}$-adic local systems $\mathbb{L}$ on $X_{K}$ for which there exist "associated $F$-isocrystals" $\mathcal{E}$ on $X_{k}$ (see [F2]). Faltings's strategy was to define a new cohomology theory associated to $X$ and to prove that it calculated both the left hand side (via the theory of almost étale extensions) and the right hand side of conjecture 1.1. Let us be more precise: we suppose that $X$ is geometrically connected and denote by $\eta=\operatorname{Spec}(\mathbb{K})$ a geometric generic point of $X_{\bar{K}}$. Let $X \bullet \longrightarrow X$ be an étale hyper-covering of $X$ by "small affine schemes over $\mathcal{O}_{K}$ ". If $X_{i}=\operatorname{Spec}\left(R_{i}\right)$ ( $i$ is a multi-index) let $\bar{R}_{i}$ be the maximal normal extension of $R_{i}$ in $\mathbb{K}$ such that $\bar{R}_{i}\left[p^{-1}\right]$ is the union of finite and étale extensions of $R_{i}\left[p^{-1}\right]$. By $B_{\text {cris }}^{\nabla}\left(\bar{R}_{i}\right)$ we denote the relative Fontaine ring $B_{\text {cris }}$ constructed

using the pair $\left(R_{i}, \bar{R}_{i}\right)$ (in [F2] this ring is denoted $B_{\text {cris }}\left(R_{i}\right)$ ). If $\Delta_{i}=\pi_{1}\left(X_{i, \bar{K}}, \eta\right)$ consider the double complex $K^{\bullet \bullet}(\mathbb{L}):=C^{\bullet}\left(\Delta_{\bullet}, \mathbb{L}_{\eta} \otimes B_{\text {cris }}^{\nabla}\left(\bar{R}_{\bullet}\right)\right)$, where $C^{\bullet}(\Delta,-)$ is the standard chain complex computing continuous group cohomology of $\Delta$. The new cohomology with coefficients 
in $\mathbb{L}$ defined by Faltings is the cohomology of the total complex $K^{\bullet \bullet}(\mathbb{L})$ (or rather the limit of such over all hyper-coverings.)

Faltings' cohomology theory seemes easier to handle than the syntomic cohomology but there are two inconveniences related to it. One is conceptual: the association $U=\operatorname{Spec}(R) \longrightarrow B_{\text {cris }}^{\nabla}(\bar{R})$ is not a sheaf and no geometric interpretation of the above defined cohomology theory is given in terms of sheaf cohomology. The second inconvenience is that in order to prove the isomorphism of the new cohomology theory of $\mathbb{L}$ with the crystalline cohomology of the associated $F$-isocrystal tensored with $B_{\text {cris }}$ one had to prove that this new cohomology theory satisfied Poincaré duality compatible with the known Poincaré dualities on the two sides of 1.1. This complicates the proof of the crystalline comparison conjecture and limits the applications of these ideas to proper schemes, or complements of a normal crossing divisor in a proper scheme.

To finish our history of "comparison isomorphisms", K. Kato in [Ka] adapted the proof in [FM] to schemes over $K$ with semi-stable reduction by the systematic use of log structures (using results in $[\mathrm{HK}]$ ) and proved the "semi-stable conjecture" for trivial coefficients and under the assumption that $1+2 \operatorname{dim}\left(X_{K}\right)$ is less than $p$. Finally T. Tsuji was able to circumvent the technical difficulties related to syntomic and $\log$ syntomic cohomology and proved both the crystalline and semi-stable conjectures for trivial coefficients in [T]. Next, Faltings extended his results to open varieties over $K$ with semi-stable reduction in [F3] and W. Niziol re-proved in [N] the crystalline and semistable conjectures for trivial coefficients using a new idea namely a comparison isomorphism in $K$-theory. Recently Go Yamashita gave a new proof of the comparison isomorphism for open varieties over $K$ with semi-stable reduction and trivial coefficients using syntomic methods (see $[\mathrm{Y}]$ ).

The new point of view introduced in the present article is the systematic use of a topos which we call "Faltings's topos" associated to $X$ and of certain new ("ind-continuous") sheaves of rings $\mathbb{B}_{\text {cris }}^{\nabla}$ and $\mathbb{B}_{\text {cris }}$ in it. Faltings's topos is the category of sheaves on a certain site which we denote $\mathfrak{X}$ (for a more precise definition see the sections 1.1 and 2.1). Despite the suggestion of the notations, the sections of $\mathbb{B}_{\text {cris }}^{\nabla}$ are not the rings $B_{\text {cris }}^{\nabla}(\bar{R})$ used by Faltings (for the precise relationship between the two see the next section.) If $\mathbb{L}$ is a $\mathbb{Q}_{p}$-local system on $X_{K}$ then $\mathbb{L}$ may be viewed as a sheaf on $\mathfrak{X}$ and we have:

a) $\mathrm{H}^{i}\left(\mathfrak{X}, \mathbb{L} \otimes_{\mathbb{Q}_{p}} \mathbb{B}_{\text {cris }}^{\nabla}\right)$ is Faltings' cohomology associated to $\mathbb{L}$ as above. Thus the present theory gives a geometric interpretation of Faltings's construction.

b) In this setting one may attach to $\mathbb{L}$ in a geometric way a sheaf on $X$ with a connection and thus define crystalline local systems on $X_{K}^{\mathrm{et}}$ and their associated $F$-isocrystals.

c) We also provide a new idea of the proof of the comparison isomorphism. The main reason the comparison isomorphism in the algebraic setting over $K$ fails to follow the classical pattern over the complex numbers is because the de Rham complex of sheaves of $X$

$$
\mathcal{O}_{X} \stackrel{d_{X}}{\longrightarrow} \Omega_{X / \mathcal{O}_{K}}^{1} \stackrel{d_{X}}{\longrightarrow} \Omega_{X / \mathcal{O}_{K}}^{2} \stackrel{d_{X}}{\longrightarrow} \cdots
$$

is not exact (i.e. there is no algebraic Poincaré lemma). Let us just mention that even if we replace $X\left(\right.$ or $\left.X_{K}\right)$ by the rigid analytic variety $X^{\text {rig }}$ associated to $X_{K}$ its de Rham complex is still not exact. But if we now pass to the finer topology $\mathfrak{X}$ and remark that the sheaf $\mathbb{B}_{\text {cris }}$ is a sheaf of $\mathcal{O}_{X}$-modules with a connection $\nabla$ such that $\mathbb{B}_{\text {cris }}^{\nabla}$ is its sheaf of horizontal sections we have an exact sequence of sheaves on $\mathfrak{X}$ :

$$
0 \longrightarrow \mathbb{B}_{\text {cris }}^{\nabla} \longrightarrow \mathbb{B}_{\text {cris }} \stackrel{\nabla}{\longrightarrow} \mathbb{B}_{\text {cris }} \otimes_{\mathcal{O}_{X}} \Omega_{X / \mathcal{O}_{K}}^{1} \stackrel{\nabla}{\longrightarrow} \mathbb{B}_{\text {cris }} \otimes_{\mathcal{O}_{X}} \Omega_{X / \mathcal{O}_{K}}^{2} \stackrel{\nabla}{\longrightarrow} \cdots
$$


In other words working on the site $\mathfrak{X}$ and after tensoring with the sheaf of periods $\mathbb{B}_{\text {cris }}$ the de Rham complex of $X$ becomes exact and a resolution of the sheaf $\mathbb{B}_{\text {cris }}^{\nabla}$. Now an acyclicity property of this resolution (for a more precise formulation see the next section) permits the calculation of the cohomology on $\mathfrak{X}$ of the sheaf $\mathbb{L} \otimes \mathbb{B}_{\text {cris }}^{\nabla}$ as the hyper-cohomology of the de Rham complex on $X$ of the associated $F$-isocrystal without any use of Poincaré duality. Therefore these results extend to $p$-adic formal schemes. In the next sub-section we present a more precise description of the article.

\subsection{Description of the paper}

Let $X$ denote either a smooth scheme over $\mathcal{O}_{K}$ or a smooth $p$-adic formal scheme over $\mathcal{O}_{K}$ with special fiber denoted $\bar{X}$. If $X$ is a scheme we denote its generic fiber by $X_{K}$ and if $X$ is a formal scheme we denote its rigid analytic generic fiber $X^{\text {rig }}$, also by $X_{K}$.

Let us define (in the algebraic setting) the category $E_{X_{\bar{K}}}$ whose objects are pairs $(\mathcal{U}, \mathcal{W})$ where $\mathcal{U} \longrightarrow X$ is an étale morphism, $\mathcal{W} \longrightarrow \mathcal{U}_{\bar{K}}$ is a finite étale morphism and the morphisms between two pairs are pairs of morphisms satisfying natural compatibilities. G. Faltings defined in [F3. a certain topology on $E_{X_{\bar{K}}}$ but as recently noticed by A. Abbes a fundamental error occurred in that construction namely to put is as directly as possible, the topos defined in [F3] is not the category of sheaves on the topology he defines there (see section 2.1 for a counterexample.) A salvage was suggested in a letter of P. Deligne to L. Illusie in 1995 raising a series of questions related to section 3.4 in [I]. He pointed out that the correct definition of Faltings's topos should be as a certain "topos fleché". The general theory of these topoi was further developed by O. Gabber, L. Illusie, F. Orgogozo and was implemented in the case of interest to us by A. Abbes. We chose not to follow this direction and fix the problem in an equivalent way here (see also [Err]) by defining a different pre-topology, which we call $\mathrm{PT}_{X_{\bar{K}}}$ on the category $E_{X_{\bar{K}}}$. See section 2.1 for the precise statements and the definition in the formal context.

There is a fundamental operation on sheaves and continuous sheaves of abelian groups on $\mathrm{PT}_{X_{\bar{K}}}$ called localization and defined as follows. Suppose that $\mathcal{F}$ is a continuous sheaf on $\mathrm{PT}_{X_{\bar{K}}}$ (let us recall that such an object is a projective system $\left\{\mathcal{F}_{n}\right\}_{n}$ of $p$-power torsion sheaves on $\mathfrak{X}$ ) and let $\mathcal{U}=\operatorname{Spec}\left(R_{\mathcal{U}}\right)$ be an affine connected object of $X^{\text {et }}$ (by which we mean the étale site on $X)$. We fix a geometric generic point $\eta=\operatorname{Spec}(\mathbb{K})$ and denote by

$$
\mathcal{F}\left(\bar{R}_{\mathcal{U}}\right):=\lim _{\leftarrow, n} \mathcal{F}_{n}\left(\bar{R}_{\mathcal{U}}\right) \text { with } \mathcal{F}_{n}\left(\bar{R}_{\mathcal{U}}\right):=\lim _{\rightarrow, S} \mathcal{F}_{n}(\mathcal{U}, \operatorname{Spec}(S)),
$$

where in the inductive limit $S$ runs over all $R_{\mathcal{U}} \otimes_{\mathcal{O}_{K}} K$-sub algebras of $\mathbb{K}$ which are finite and étale. Let us remark that $\mathcal{F}\left(\bar{R}_{\mathcal{U}}\right)$ is a continuous representation of the fundamental group $\pi_{1}^{\text {alg }}\left(\mathcal{U}_{K}, \eta\right)$.

We have natural functors $v: X^{\text {et }} \longrightarrow \mathfrak{X}, u: \mathfrak{X} \longrightarrow X_{\bar{K}}^{\text {et }}$ defined (in the algebraic setting) as follows: $v(\mathcal{U})=\left(\mathcal{U}, \mathcal{U}_{\bar{K}}\right)$ and respectively $u(\mathcal{U}, \mathcal{W})=\mathcal{W}$. The functors $v^{*}$ and $u_{*}$ allow us to view sheaves on $X^{\mathrm{et}}$ and respectively on $X_{\bar{K}}^{\mathrm{et}}$ as sheaves on $\mathfrak{X}$. The functor $v_{*}$ is left exact and its right derived functors are central to our theory. We have the following result proved in [AI] describing these functors in terms of localizations.

Theorem 1.2 ([AI], theorem 6.12). Let $\mathcal{F}$ be a continuous sheaf on $\mathfrak{X}$ satisfying certain conditions (Assumptions 6.10 in $[\mathrm{AI}]$ ). Then for all $i \geq 0, R^{i} v_{*} \mathcal{F}$ are the sheaves associated to the pre-sheaves on $X^{\mathrm{et}}$

$$
\mathcal{U}=\operatorname{Spec}\left(R_{\mathcal{U}}\right) \longrightarrow \mathrm{H}_{\text {cont }}^{i}\left(\pi_{1}^{\text {alg }}\left(\mathcal{U}_{\bar{K}}, *\right), \mathcal{F}\left(\bar{R}_{\mathcal{U}}\right)\right)
$$


This theorem reduces via the Leray spectral sequence the calculation of the sheaf cohomology groups $\mathrm{H}^{i}(\mathfrak{X}, \mathcal{F})$ to local calculations of fundamental group cohomology with values in localizations and sheaf cohomology on $X^{\mathrm{et}}$.

We now pass to the description of the sheaves $\mathbb{B}_{\text {cris }}^{\nabla}$ and $\mathbb{B}_{\text {cris }}$ announced at the beginning of this introduction. We call these "Fontaine sheaves" and prove that they enjoy the following properties.

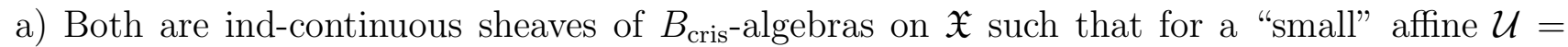
$\operatorname{Spec}\left(R_{\mathcal{U}}\right)$ the localizations $\mathbb{B}_{\text {cris }}^{\nabla}\left(\bar{R}_{\mathcal{U}}\right)$ and $\mathbb{B}_{\text {cris }}\left(\bar{R}_{\mathcal{U}}\right)$ are respectively isomorphic to the rings $B_{\text {cris }}^{\nabla}\left(\bar{R}_{\mathcal{U}}\right)$ and $B_{\text {cris }}\left(\bar{R}_{\mathcal{U}}\right)$ defined in [F2] and [Bri].

b) $\mathbb{B}_{\text {cris }}^{\nabla}$ is endowed with a filtration $\mathrm{Fil}^{\bullet}\left(\mathbb{B}_{\text {cris }}^{\nabla}\right)$ by sub-sheaves and a Frobenius endomorphism.

c) $\mathbb{B}_{\text {cris }}$ is a sheaf of $\mathcal{O}_{X} \otimes_{\mathcal{O}_{K}} B_{\text {cris }}$-algebras, is endowed with a filtration Fil ${ }^{\bullet}\left(\mathbb{B}_{\text {cris }}\right.$ ) by sub-sheaves, a Frobenius endomorphism and a quasi-nilpotent and integrable connection $\nabla$ such that

i) $\nabla$ satisfies the Griffith transversality property.

ii) $\mathbb{B}_{\text {cris }}^{\nabla}$ is exactly the sub-sheaf of $\mathbb{B}_{\text {cris }}$ of horizontal sections for $\nabla$.

d) For $i \geq 1$ we have $R^{i} v_{*} \mathbb{B}_{\text {cris }}=0$.

We remark that property $d$ ) above is a deep result stating that the sheaf $\mathbb{B}_{\text {cris }}$ is acyclic for the functor $v_{*}$. It is a consequence of theorem 1.2 and the results in [AB]. As we now have Fontaine sheaves on $\mathfrak{X}$ we can start developing a Fontaine theory with sheaves. To start let $\mathbb{L}$ denote a locally constant $\mathbb{Q}_{p}$-sheaf on $X_{K}^{\text {et }}$ which, let us recall, we view via base change and $u_{*}$ as a sheaf on $\mathrm{PT}_{X_{\bar{K}}}$. We define $\mathbb{D}_{\text {cris }}^{\text {geo }}(\mathbb{L}):=v_{*}\left(\mathbb{L} \otimes \mathbb{B}_{\text {cris }}\right)$. It is a sheaf of $\mathcal{O}_{X_{K}} \otimes_{K} B_{\text {cris-modules on }} X_{K}^{\text {et }}$ endowed with a filtration, Frobenius endomorphism, quasi-nilpotent and integrable connection and a continuous $G_{K}$-action. Here $\mathcal{O}_{X_{K}}$ denotes the sheaf $\mathcal{O}_{X}\left[p^{-1}\right]$ on $X$. We set $\mathbb{D}_{\text {cris }}^{\text {ar }}(\mathbb{L}):=$ $\left(\mathbb{D}_{\text {cris }}^{\text {geo }}(\mathbb{L})\right)^{G_{K}}$.

Definition 1.3. We say that $\mathbb{L}$ is a crystalline sheaf on $X_{K}^{\text {et }}$ if

- $\mathbb{D}_{\text {cris }}^{\text {ar }}(\mathbb{L})$ is a coherent sheaf of $\mathcal{O}_{X_{K}}$-modules on $X_{K}^{\mathrm{et}}$.

- The natural morphism $\mathbb{D}_{\text {cris }}^{\text {ar }}(\mathbb{L}) \otimes_{\mathcal{O}_{X}} \mathbb{B}_{\text {cris }} \longrightarrow \mathbb{L} \otimes_{\mathbb{Q}_{p}} \mathbb{B}_{\text {cris }}$ is an isomorphism.

The definition 1.3 is the sheaf theoretic analogue of the usual definition of crystalline representations in $p$-adic Hodge theory. We prove that it coincides with the notions of "locally crystalline representations" in the relative setting due to [Bri] and with that of "associated sheaves" due to Faltings. If $X$ is a formal scheme as at the beginning of this section and $\mathbb{L}$ is a crystalline sheaf on $X_{K}^{\text {et }}$ then $\mathbb{D}_{\text {cris }}^{\text {ar }}(\mathbb{L})$ is a filtered convergent $F$-isocrystal on $X_{K}^{\text {et }}$ in the sense of [B3] and $\mathbb{D}_{\text {cris }}^{\text {geo }}(\mathbb{L}) \cong \mathbb{D}_{\text {cris }}^{\text {ar }}(\mathbb{L}) \otimes_{K} B_{\text {cris. }}$. We remark that in the recent preprint [T1] T. Tsuji developed systematically a theory of crystalline étale local systems on schemes $X_{K}$ in the case where $X$ is the complement of a divisor with normal crossings in a proper formal scheme over $\mathcal{O}_{K}$ with semi-stable special fiber and such that the horizontal divisor has normal crossings also with the special fiber. The paper uses different methods and does not contain comparison isomorphisms. If $X$ is a smooth formal scheme (and the horizontal divisor is trivial) our notion of a crystalline étale local system on $X_{K}$ coincides with the one in [T1].

We can now list the main result of this paper.

Theorem 1.4. Suppose that $X$ is a smooth p-adic formal scheme over $\mathcal{O}_{K}$ and let $\mathbb{L}$ be a crystalline sheaf on $X_{K}^{\mathrm{et}}$. For every $i \geq 0$ we have a natural isomorphism of $\delta$-functors with 


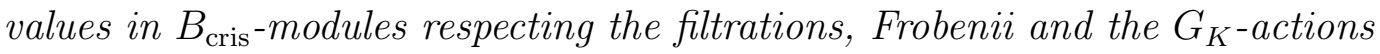

$$
\mathrm{H}^{i}\left(\mathfrak{X}, \mathbb{L} \otimes \mathbb{B}_{\text {cris }}^{\nabla}\right) \cong \mathrm{H}_{\text {cris }}^{i}\left(\bar{X}, \mathbb{D}_{\text {cris }}^{\text {geo }}(\mathbb{L})\right)
$$

The theorem 1.4 has two main applications, one which is the comparison isomorphism for smooth proper schemes over $\mathcal{O}_{K}$ (theorem 1.5 below) and the other which is an application to modular forms, more precisely an overconvergent Eichler-Shimura isomorphism (see [AIS]).

Theorem 1.5. Suppose that $X$ is a smooth proper scheme over $\mathcal{O}_{K}$ and $\mathbb{L}$ is a crystalline sheaf on $X_{K}^{\mathrm{et}}$. For every $i \geq 0$ we have a canonical isomorphism of $\delta$-functors with values in

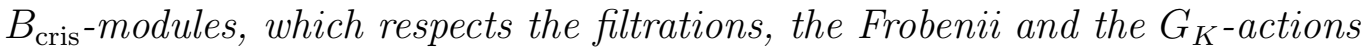

$$
\mathrm{H}^{i}(X \overline{\mathrm{et}}, \mathbb{L}) \otimes_{\mathbb{Q}_{p}} B_{\text {cris }} \cong \mathrm{H}_{\text {cris }}^{i}\left(\bar{X}, \mathbb{D}_{\text {cris }}^{\text {ar }}(\mathbb{L})\right) \otimes_{K} B_{\text {cris }}
$$

The theorem 1.5 is a consequence of theorem 1.4 and of the following two results:

- If $X$ is a smooth, proper formal scheme over $\mathcal{O}_{K}$ then we have an isomorphism of filtered, Frobenius modules: $\mathrm{H}_{\text {cris }}^{i}\left(\bar{X}, \mathbb{D}_{\text {cris }}^{\text {geo }}(\mathbb{L})\right) \cong \mathrm{H}_{\text {cris }}^{i}\left(\bar{X}, \mathbb{D}_{\text {cris }}^{\text {ar }}(\mathbb{L})\right) \otimes_{K} B_{\text {cris }}$.

and

- The natural morphism of sheaves on $\mathfrak{X}, \mathbb{L} \longrightarrow \mathbb{L} \otimes \mathbb{B}_{\text {cris }}^{\nabla}$ induces for every $i \geq 0$ canonical isomorphisms as $B_{\text {cris }}$-modules respecting all the structure $\mathrm{H}^{i}\left(X \frac{\mathrm{et}}{K}, \mathbb{L}\right) \otimes_{\mathbb{Q}_{p}} B_{\text {cris }} \cong \mathrm{H}^{i}\left(\mathfrak{X}, \mathbb{L} \otimes_{\mathbb{Z}_{p}}\right.$ $\mathbb{B}_{\text {cris }}^{\nabla}$.

This last isomorphism which is also proved in [F3] as being one of the central and deep results of that paper is in our theory an elementary consequence of theorem 1.4 and of the criterion for "admisibility" of filtered, Frobenius modules in [CF].

Let us remark that in lemma 3.14 we prove that $\mathbb{L}$ is a crystalline sheaf on $X_{K}^{\text {et }}$ if and only if $\mathbb{L}$ and $\mathbb{D}_{\text {cris }}^{\text {ar }}(\mathbb{L})$ are associated in Faltings's sense. This and the fact that $H^{i}\left(\mathfrak{X}, \mathbb{L} \otimes \mathbb{B}_{\text {cris }}^{\nabla}\right)$ is naturally isomorphic to the $i$-th cohomology group defined by Faltings shows that the comparison isomorphism of theorem 1.5 is the same as the one defined by Faltings, and hence it is the same as all the other period maps defined in the literature.

Finally, in a future work we are planning to show how to produce examples of crystalline sheaves and how to explicitly calculate their $\mathbb{D}_{\text {cris }}^{\text {ar }}$. More precisely let $X$ and $Y$ denote smooth $p$-adic formal schemes over $\mathcal{O}_{K}$ and suppose that $f: X \longrightarrow Y$ is a smooth proper morphism which is algebrizable Zariski locally on $Y$. We believe that we would be able to prove:

Theorem 1.6. Let us suppose that $\mathbb{M}$ is a crystalline sheaf on $X_{K}^{\mathrm{et}}$ and for every $i \geq 0$ let us denote by $\mathbb{L}_{i}:=\mathrm{R}^{i} f_{\mathrm{et}, *} \mathbb{M}$. Then $\mathbb{L}_{i}$ is a crystalline sheaf on $Y_{K}^{\mathrm{et}}$ and we have an isomorphism $\mathbb{D}_{\text {cris,Y }}^{\text {ar }}\left(\mathbb{L}_{i}\right) \cong \mathbb{R}^{i} f_{\text {cris,* }}\left(\mathbb{D}_{\text {cris,X }}^{\text {ar }}(\mathbb{M}) \otimes \Omega_{X / Y}^{\bullet}\right)$ of $\delta$-functors with values in the category of filtered convergent $F$-isocrystals on $Y_{k}$.

The reader will remark throughout the paper the presence of an auxiliary field $M$ which is an extension of $K$ contained in $\bar{K}$ and which indexes all the objects appearing: $\mathfrak{X}_{M}, \mathbb{A}_{\text {cris, },}, \mathbb{A}_{\text {cris, } M}$ etc. If $M$ is a finite extension of $K$, this allows us to prove the theorems above also for the base change of $X$ to the ring of integers of $M$. Equivalently the above results are valid without 
assuming that $K$ is absolutely unramified but under the hypothesis that $X$ (and the morphism $f: X \rightarrow Y$ in [1.6) is defined over $\mathbb{W}(k)$. Since the notations become more complicated and possibly obscure some of the simple ideas present in the proofs we have chosen to sketch these ideas in the introduction in the simplified assumption that $K$ is unramified.

We would also like to point out that the methods presented here seem suitable for pursuing further inquiries into this problem. Namely we have already worked out the comparison theorems for schemes and formal schemes over the ring of integers of a finite extension $K$ of $\mathbb{Q}_{p}$ (hence removing the "unramified-ness" assumption present in this paper) with semi-stable special fibers and hope to be able to report on these results soon. Moreover we think that for smooth schemes over $\mathcal{O}_{K}$ one may replace the locally constant $\mathbb{Q}_{p}$-sheaf $\mathbb{L}$ on $X_{K}^{\text {et }}$ by a constructible $\mathbb{Q}_{p}$-sheaf and obtain interesting comparison isomorphisms. We also believe that we should be able to derive integral comparison isomorphisms which would work better than the existing ones.

Acknowledgements We thank Ahmed Abbes for pointing out the error in [F3] (see the section 2.1) and for many helpful discussions and email exchanges on this subject and Luc Illusie for kindly providing us with copies of his correspondence with Deligne on Faltings' topology. We are grateful to the two referees for the careful reading of the paper and for pointing out some mistakes and suggesting ways to remedy them. Some of their remarks have been incorporated in the article (e.g. see remark 2.12.) Finally, part of the work on this article was done while both authors were guests of L'Institut Henri Poincaré, Paris during the Galois semester 2010. We thank this institution for its hospitality.

\subsection{Notations}

Let $p>0$ be a prime integer, $\mathcal{O}_{K}$ a complete discrete valuation ring with fraction field $K$ and perfect residue field $k$. Fix an algebraic closure $K \subset \bar{K}$, let $\bar{k}$ denote its residue field and $\mathcal{O}_{\bar{K}}$ the normalization of $\mathcal{O}_{K}$ in $\bar{K}$. Write $G_{K}$ for the Galois group of $\bar{K}$ over $K$. Fix a field extension $K \subset M \subset \bar{K}$. We write $M_{0} \subseteq M$ for the maximal absolutely unramified subfield of $M$ and $\mathcal{O}_{M_{0}}$ for its ring of integers.

The following notations will be used throughout the paper (some of the objects denoted here will be defined in this very section and the rest in the next sections):

- Rings:

$W_{n}:=\mathbb{W}_{n}\left(\mathcal{O}_{\bar{K}} / p \mathcal{O}_{\bar{K}}\right), A_{\text {inf }}^{+}:=A_{\text {inf }}^{+}\left(\mathcal{O}_{\bar{K}}\right), A_{\text {inf }}:=A_{\text {inf }}\left(\mathcal{O}_{\bar{K}}\right), A_{\text {cris }, \mathrm{n}}:=A_{\text {cris }, \mathrm{n}}\left(\mathcal{O}_{\bar{K}}\right), A_{\text {cris }, \mathrm{n}}^{\prime}:=$ $A_{\text {cris }, \mathrm{n}}^{\prime}\left(\mathcal{O}_{\bar{K}}\right), A_{\text {cris }}:=A_{\text {cris }}\left(\mathcal{O}_{\bar{K}}\right)=A_{\text {cris }}^{\prime}\left(\mathcal{O}_{\bar{K}}\right), B_{\text {cris }}:=B_{\text {cris }}\left(\mathcal{O}_{\bar{K}}\right)$.

- Sheaves on $\mathfrak{X}_{M}$ :

$\mathbb{W}_{n, M}:=\mathbb{W}_{n}\left(\mathcal{O}_{\mathfrak{X}_{M}} / p \mathcal{O}_{\mathfrak{X}_{M}}\right), \mathbb{W}_{n}:=\mathbb{W}_{n, \bar{K}}, \mathbb{A}_{\text {inf,M }}^{+}:=\left\{\mathbb{W}_{n, M}\right\}_{n}, \mathbb{A}_{\text {inf }}:=\left\{\mathbb{W}_{n}\right\}_{n}$

We recall a few facts regarding the properties and (one of) the constructions of $A_{\text {cris }}$ needed in the sequel. For details we refer to [Fo, $\S 1 \& \S 2]$. Choose a compatible sequence of roots $\left(p^{1 / p^{n-1}}\right)_{n \geq 1}$ in $\mathcal{O}_{\bar{K}}$ (compatible means that $\left(p^{1 / p^{n}}\right)^{p}=p^{1 / p^{n-1}}$, for all $\left.n \geq 1\right)$. For every $n \in \mathbb{N}$ we have a ring homomorphism $\theta_{n}: W_{n}:=\mathbb{W}_{n}\left(\mathcal{O}_{\bar{K}} / p \mathcal{O}_{\bar{K}}\right) \longrightarrow \mathcal{O}_{\bar{K}} / p^{n} \mathcal{O}_{\bar{K}}$ given by $\left(s_{0}, \ldots, s_{n-1}\right) \mapsto$ $\sum_{i=0}^{n-1} p^{i} \tilde{s}_{i}^{p^{n-1-i}}$ where $\tilde{s}_{i} \in \mathcal{O}_{\bar{K}} / p^{n} \mathcal{O}_{\bar{K}}$ is a lift of $s_{i}$ for every $i$. Write $\varphi$ for Frobenius on $W_{n}$. Denote by $\widetilde{p}_{n}:=\left[p^{1 / p^{n-1}}\right] \in W_{n}$ the Teichmüller lift of $p^{1 / p^{n-1}} \in \mathcal{O}_{\bar{K}} / p \mathcal{O}_{\bar{K}}$. Let $\xi_{n}:=\widetilde{p}_{n}-p \in W_{n}$, 
then $\xi_{n}$ generates $\operatorname{Ker}\left(\theta_{n}\right)$. Denote by $A_{\text {cris,n }}$ the $\mathbb{W}(k)$-DP-envelope of $W_{n}$ with respect to the ideal $\operatorname{Ker}\left(\theta_{n}\right)$ (where $\mathbb{W}(k)$-DP-envelope means that the divided powers are compatible with the standard divided powers on $\left.p \mathbb{W}_{n}(k)\right)$. Note that $A_{\text {cris,n }}$ is naturally endowed with an action of $G_{K}$. Denote by $\operatorname{Ker}\left(\theta_{n}\right)^{\text {DP }}$ the PD-ideal on $A_{\text {cris }, \mathrm{n}}$. Note that $\varphi\left(\xi_{n}\right)=\varphi\left(\widetilde{p}_{n}-p\right)=\left(\widetilde{p}_{n}^{p}-p^{p}\right)+\left(p^{p}-p\right)$. Since $\widetilde{p}_{n}^{p}-p^{p} \in \operatorname{Ker}\left(\theta_{n}\right)$ and $p$ admits divided powers in $A_{\text {cris }, \mathrm{n}}$, also $\varphi\left(\xi_{n}\right)$ does. Thus Frobenius on $W_{n}$ extends to an operator called Frobenius and denoted by $\varphi$, on $A_{\text {cris,n }}$.

Let $\underline{\mathcal{R}}\left(\mathcal{O}_{\bar{K}}\right):=\lim \mathcal{O}_{\bar{K}} / p \mathcal{O}_{\bar{K}}$ where the inverse limit is taken with respect to Frobenius. Put $A_{\text {inf }}^{+}:=\mathbb{W}\left(\underline{\mathcal{R}}\left(\mathcal{O}_{\bar{K}}\right)\right) \cong \lim _{\infty \leftarrow n} W_{n}$ where the latter inverse limit is taken with respect to the map $u_{n+1}: W_{n+1} \longrightarrow W_{n}$ defined by the natural projection composed with Frobenius. Remark that the maps $\theta_{n}$ are compatible i. e., $\theta_{n}=\theta_{n+1} \circ u_{n+1}$, that the sequence $\xi:=\left\{\xi_{n}\right\}_{n}$ is compatible i.e., $u_{n+1}\left(\xi_{n+1}\right)=\xi_{n}$ for all $n \geq 0$, and that $\operatorname{Ker}(\theta)$ is generated by $\xi$. Denote by $A_{\text {cris }}:=\lim _{\infty \leftarrow n} A_{\text {cris,n }}$. It is the $p$-adic completion of the $\mathbb{W}(k)$-DP-envelope of $A_{\text {inf }}^{+}$with respect to the ideal $\operatorname{Ker}(\theta)$. We then have

$$
A_{\text {cris }}=A_{\text {inf }}^{+}\{\langle\xi\rangle\}=A_{\text {inf }}^{+}\left\{\delta_{0}, \delta_{1}, \ldots\right\} /\left(p \delta_{0}-\xi^{p}, p \delta_{m+1}-\delta_{m}^{p}\right)_{m \geq 0}
$$

where $\delta_{i}=\gamma^{i+1}(\xi)$ and $\gamma$ is the application on the kernel of $\theta$ on $A_{\text {cris }}$ given by $z \mapsto(p-1) ! z^{[p]}$; cf. [Bri, Prop. 6.1.2]. Note that $\mathbb{W}_{n}\left(\underline{\mathcal{R}}\left(\mathcal{O}_{\bar{K}}\right)\right) \cong A_{\text {inf }}^{+} / p^{n} A_{\text {inf }}^{+}$since $A_{\text {inf }}^{+}=\mathbb{W}\left(\underline{\mathcal{R}}\left(\mathcal{O}_{\bar{K}}\right)\right)$ and $\underline{\mathcal{R}}\left(\mathcal{O}_{\bar{K}}\right)=\lim _{\leftarrow} \mathcal{O}_{\bar{K}} / p \mathcal{O}_{\bar{K}}$ is a perfect ring by construction.

Lemma 1.7. The kernel of the ring homomorphism $q_{n}: \mathbb{W}_{n}\left(\underline{\mathcal{R}}\left(\mathcal{O}_{\bar{K}}\right)\right) \longrightarrow \mathbb{W}_{n}\left(\mathcal{O}_{\bar{K}} / p \mathcal{O}_{\bar{K}}\right)$ induced by $\bar{q}_{n}$ is the ideal generated by $\left\{[\widetilde{p}]^{p^{n}}, V\left([\widetilde{p}]^{p^{n}}\right), V^{2}\left([\widetilde{p}]^{p^{n}}\right), \ldots, V^{n-1}\left([\widetilde{p}]^{p^{n}}\right)\right\}$. In particular

$$
A_{\text {cris }} / p^{n} A_{\text {cris }} \cong W_{n}\left[\delta_{0}, \delta_{1}, \ldots\right] /\left(p \delta_{0}-\xi_{n+1}^{p}, p \delta_{m+1}-\delta_{m}^{p}\right)_{m \geq 0}
$$

via the map which sends $\delta_{i} \mapsto \delta_{i}$ and induces on $\mathbb{W}_{n}\left(\underline{\mathcal{R}}\left(\mathcal{O}_{\bar{K}}\right)\right)$ the morphism $q_{n}: \mathbb{W}_{n}\left(\underline{\mathcal{R}}\left(\mathcal{O}_{\bar{K}}\right)\right) \rightarrow$ $W_{n}$ associated to $\bar{q}_{n}$.

Proof. We prove the first claim. We have $\widetilde{p}^{p^{n}}=(\xi+p)^{p^{n}} \equiv \xi^{p^{n}} \bmod p^{n} A_{\text {inf }}^{+}$and $\xi^{p^{n}}=$ $p^{p^{n-1}} \delta_{0}^{p^{n-1}}=0 \bmod p^{n} A_{\text {inf }}^{+}$. The kernel of the projection $\bar{q}_{n}: \underline{\mathcal{R}}\left(\mathcal{O}_{\bar{K}}\right)=\lim _{\leftarrow} \mathcal{O}_{\bar{K}} / p \mathcal{O}_{\bar{K}} \rightarrow \mathcal{O}_{\bar{K}} / p \mathcal{O}_{\bar{K}}$ on the $n+1$-th factor of the limit is generated by $\widetilde{p}^{p^{n}}$. This proves the lemma for $n=1$. The general case follows by induction on $n$ using the exact sequence

$$
0 \longrightarrow \mathbb{W}_{n-1}\left(\underline{\mathcal{R}}\left(\mathcal{O}_{\bar{K}}\right)\right) \stackrel{V}{\longrightarrow} \mathbb{W}_{n}\left(\underline{\mathcal{R}}\left(\mathcal{O}_{\bar{K}}\right)\right) \longrightarrow \mathbb{W}_{1}\left(\underline{\mathcal{R}}\left(\mathcal{O}_{\bar{K}}\right)\right) \longrightarrow 0 .
$$

Now let us recall that $[\widetilde{p}]^{p^{n}}=0$ in $A_{\text {cris }} / p^{n} A_{\text {cris }}$ and similarly, for every $0 \leq i \leq n-1$ we have $V^{i}\left([\widetilde{p}]^{p^{n}}\right)=p^{i}[\widetilde{p}]^{p^{n-i}}=0$ in $A_{\text {cris }} / p^{n} A_{\text {cris }}$. Then the second claim follows.

In particular $A_{\text {cris }} / p^{n} A_{\text {cris }}$ is the $\mathbb{W}(k)$-DP envelope of $W_{n}$ with respect to $\xi_{n+1} W_{n}=\operatorname{Ker}\left(\theta_{n}\right.$ 。 $\varphi)$. We then get a surjective map of DP algebras

$$
q_{n}: A_{\text {cris }} / p^{n} A_{\text {cris }} \longrightarrow A_{\text {cris }, \mathrm{n}}
$$

sending $\xi_{n+1}^{[i]} \mapsto \xi_{n}^{[i]}$ and inducing Frobenius on $W_{n}$. We also have a map

$$
u_{n}: A_{\text {cris }, \mathrm{n}+1} \longrightarrow A_{\text {cris }} / p^{n} A_{\text {cris }}
$$


sending $\xi_{n+1}^{[i]} \mapsto \xi_{n+1}^{[i]}$ and inducing the natural projection $W_{n+1} \rightarrow W_{n}$.

We introduce the following ideal $\mathbb{I} \subset A_{\text {cris }}$. Let $\left\{\zeta_{n}\right\}_{n \in \mathbb{N}}$ be a compatible system of primitive $p^{n}$-th roots of unity: $\zeta_{2} \neq 1$ and $\zeta_{n+1}^{p}=\zeta_{n}$. It defines an element $\varepsilon=\left(1, \zeta_{2}, \zeta_{3}, \ldots\right) \in \mathcal{R}\left(\mathcal{O}_{\bar{K}}\right)$. Let $[\varepsilon] \in A_{\text {inf }}^{+}$be its Teichmüller lift. Let $\mathbb{I}$ be the ideal generated by $\left\{\varphi^{-n}([\varepsilon])-1\right\}_{n \in \mathbb{N}}$ and the Teichmüller lifts $[x]$ of elements $x=\left(x_{0}, x_{1}, \ldots\right) \in \mathcal{R}\left(\mathcal{O}_{\bar{K}}\right)$ such that $x_{0}$ lies in the maximal ideal of $\mathcal{O}_{\bar{K}} / p \mathcal{O}_{\bar{K}}$. It is proven in [Bri, Lem. 6.3.1] that $\mathbb{I}^{2}=\mathbb{I} \bmod p^{n} A_{\text {cris }}$. Since $\theta([\varepsilon]-1)=0$, the element $[\varepsilon]-1$ admits divided powers. In $A_{\text {cris }}$ we have the following important element

$$
t:=\log ([\varepsilon])=\sum_{n=1}^{\infty}(n-1) !([\varepsilon]-1)^{[n]}
$$

We have $\varphi(t)=p t$ and for $\sigma \in G_{K}, \sigma(t)=\chi(\sigma) t$ where $\chi: g_{K} \rightarrow \mathbb{Z}_{p}^{*}$ is the cyclotomic character defined by $\sigma\left(\zeta_{n}\right)=\zeta_{n}^{\chi(\sigma)}$ for every $n \in \mathbb{N}$. Put $B_{\text {cris }}:=A_{\text {cris }}[1 / t]$. Since $t$ lies in $\operatorname{Ker}(\theta)$, it admits divided powers in $A_{\text {cris }}$ so that $t^{p}=p ! t^{[p]}$ and $p$ is invertible in $B_{\text {cris }}$. Then $B_{\text {cris }}$ is a $\mathbb{W}(\bar{k})$-algebra, endowed with an action of $G_{K}$, a Frobenius operator $\varphi$ and a separated and exhaustive filtration $\mathrm{Fil}^{r} B_{\text {cris }}:=\lim _{n \in \mathbb{N}} \mathrm{Fil}^{r+n} A_{\text {cris }} \cdot t^{-n}$ for every $r \in \mathbb{Z}$.

\section{Fontaine sheaves}

Let $X$ denote a smooth scheme over $\mathcal{O}_{K}$ or a smooth $p$-adic formal scheme topologically of finite type, over $\mathcal{O}_{K}$. In this section we introduce several sites describing their underlying categories and giving pre-topology structures i. e., for each object, we describe the covering families. The topologies underlying the sites will be the topologies generated by the given pre-topologies. See [SGAIV, §II.1] for details.

\subsection{Faltings' topos; the algebraic setting}

Let us first treat the case when $X$ is a scheme of finite type over $\mathcal{O}_{K}$. We denote by $X^{\text {et }}$ the small étale site on $X$ and by $X_{M}^{\text {fet }}$ the finite étale site of $X_{M}$. Then $\operatorname{Sh}\left(X^{\mathrm{et}}\right)$ and $\operatorname{Sh}\left(X_{M}^{\mathrm{fet}}\right)$ will denote the categories of sheaves of abelian groups on these sites, respectively.

Definition 2.1. Let $E_{X_{M}}$ be the category defined as follows

i) the objects consist of pairs $\left(g: U \longrightarrow X, f: W \longrightarrow U_{M}\right)$ such that $g$ is an étale morphism of finite type and $f$ is a finite étale morphism. We will usually denote by $(U, W)$ this object to shorten notations;

ii) a morphism $\left(U^{\prime}, W^{\prime}\right) \longrightarrow(U, W)$ in $E_{X_{M}}$ consists of a pair $(\alpha, \beta)$, where $\alpha: U^{\prime} \longrightarrow U$ is a morphism over $X$ and $\beta: W^{\prime} \longrightarrow W$ is a morphism commuting with $\alpha \otimes_{\mathcal{O}_{K}} \operatorname{Id}_{M}$.

Let us remark that the pair $\left(X, X_{M}\right)$ is a final object of $E_{X_{M}}$. Moreover, finite projective limits are representable in $E_{X_{M}}$ and, in particular, fibre products exist: the fibre product of the objects $\left(U^{\prime}, W^{\prime}\right)$ and $\left(U^{\prime \prime}, W^{\prime \prime}\right)$ over $(U, W)$ is $\left(U^{\prime} \times_{U} U^{\prime \prime}, W^{\prime} \times_{W} W^{\prime \prime}\right)$. See [Err]. 
Faltings defined in [F3, p. 214] a pre-topology on $E_{X_{M}}$ by defining a family of morphisms $\left\{\left(U_{i}, W_{i}\right) \longrightarrow(U, W)\right\}_{i \in I}$ to be a covering family if $\left\{U_{i} \longrightarrow U\right\}_{i \in I}$ is a covering in $X^{\text {et }}$ and $\left\{W_{i} \longrightarrow W\right\}_{i \in I}$ is a covering family in $X_{M}^{\mathrm{fet}}$. He then defined the presheaf $\mathcal{O}_{\mathfrak{X}}$ on $E_{X_{M}}$ by

$$
\mathcal{O}_{\mathfrak{X}}(U, W):=\text { the normalization of } \Gamma\left(U, \mathcal{O}_{U}\right) \text { in } \Gamma\left(W, \mathcal{O}_{W}\right)
$$

and stated that this was a sheaf. However, this is not true in general due to point b) of the following example. Moreover point c) below shows that even if one sheafified the presheaf $\mathcal{O}_{\mathfrak{X}}$ on Faltings' site the theory of "localizations" of sheaves, as developed later in this paper, would not work. It should be noticed, though, that even if the definition of the topology is not correct, the topos of sheaves described by Faltings coincides with the one defined in this paper.

Example 2.2. Assume that $M=\bar{K}$. Let $p>2$ be a prime and let us denote by $A:=$ $\mathbb{Z}_{p}\left[X, \frac{1}{X^{2}+p}\right]$ and $B:=\mathbb{Z}_{p}\left[X, Y, \frac{1}{X^{2}+p}\right] /\left(Y^{2}-X^{2}-p\right)$. For $i=1,2$ we define $B_{i}:=$ $B\left[\frac{1}{Y+(-1)^{i} X}\right]$ and let $f_{i}$ denote the composition of the natural $\mathbb{Z}_{p}$-algebra morphisms $A \longrightarrow$ $B \longrightarrow B_{i}$. We denote $U:=\operatorname{Spec}(A), V:=\operatorname{Spec}(B), U_{i}:=\operatorname{Spec}\left(B_{i}\right)$ and $W=W_{i}:=\operatorname{Spec}\left(B_{\bar{K}}\right)$. Fix $i \in\{1,2\}$, then we have

a) The pairs $(U, W)$ and $\left(U_{i}, W_{i}\right)$ are objects of $E_{U_{\bar{K}}}$ and if we denote by $F_{i}:(U, W) \longrightarrow$ $\left(U_{i}, W_{i}\right)$ the morphism induced by the pair $\left(f_{i}, I d\right)$, then this morphism is a coverings in Faltings' sense.

b) For the covering above the presheaf $\mathcal{O}_{\mathfrak{X}}$ does not satisfy the sheaf property.

c) Let us denote by $\mathcal{F}$ the sheaf associated to $\mathcal{O}_{\mathfrak{X}}$ on the topology defined by Faltings and by $\mathcal{G}$ the sheaf $\mathcal{F} / p \mathcal{F}$. Then the natural map: $\mathcal{O}_{\mathfrak{X}}(U, W) / p \mathcal{O}_{\mathfrak{X}}(U, W) \longrightarrow \mathcal{G}(U, W)$ is the zero map.

Proof. a) Let us observe that

$$
\bar{B}:=B / p B \cong \mathbb{F}_{p}[X, 1 / X] /((X+Y)(X-Y)) \cong \mathbb{F}_{p}[X, 1 / X] \times \mathbb{F}_{p}[X, 1 / X],
$$

and we let $\bar{V}:=\operatorname{Spec}(\bar{B}) \cong \bar{V}_{1} \amalg \bar{V}_{2}$ where we have denoted by $\bar{V}_{i} \cong \operatorname{Spec}\left(\mathbb{F}_{p}[X, 1 / X]\right)$ for $i=1,2$ the components of $\bar{V}$.

Then let us remark that $U_{i} \cong V-\bar{V}_{i}$. As $V \longrightarrow U$ is étale and surjective it follows that the morphisms induced by $f_{i}, U_{i} \longrightarrow U$ are étale and surjective. Moreover the natural $\mathbb{Z}_{p}$-algebra morphisms $B \longrightarrow B_{i}$ for $i=1,2$ induce isomorphisms as $\bar{K}$-algebras $B_{\bar{K}} \cong B_{1, \bar{K}} \cong B_{2, \bar{K}}$. Now a) follows.

b) We fix $i \in\{1,2\}$ as in the statement and we have the following commutative diagram

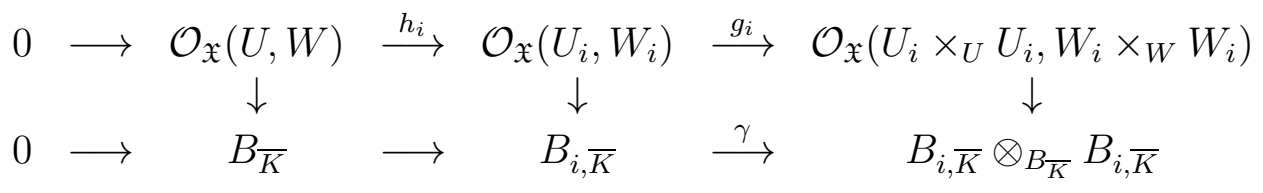

The vertical arrows in the diagram are inclusions therefore they are injective. Moreover $\gamma$ is defined by: $\gamma(b):=b \otimes 1-1 \otimes b=0$ for all $b \in B_{i, \bar{K}}$ in view of the remarks above, therefore $\gamma=0$ which implies that $g_{i}=0$. If $\mathcal{O}_{\mathfrak{X}}$ were a sheaf then the top sequence would be exact, i.e. $h_{i}$ would be an isomorphism. Thus all elements of $B_{i} \subset \mathcal{O}_{\mathfrak{X}}\left(U_{i}, W_{i}\right)$ would be integral over $A$. In particular as $B_{i}$ is a finitely generated $A$-algebra, $B_{i}$ would be finite over $A$. Since $U=\operatorname{Spec}(A)$ 
is connected the degree of $B_{i}$ as an $A$-module would be constant. But $A / p A \longrightarrow B_{i} / p B_{i}$ is an isomorphism while $B_{i, \bar{K}}$ is a free $A_{\bar{K}}$-module of rank 2 . Therefore $\mathcal{O}_{\mathfrak{X}}$ is not a sheaf.

c) As $\mathcal{F}$ is a sheaf, for each $i=1,2$ we have a commutative diagram with the bottom row exact

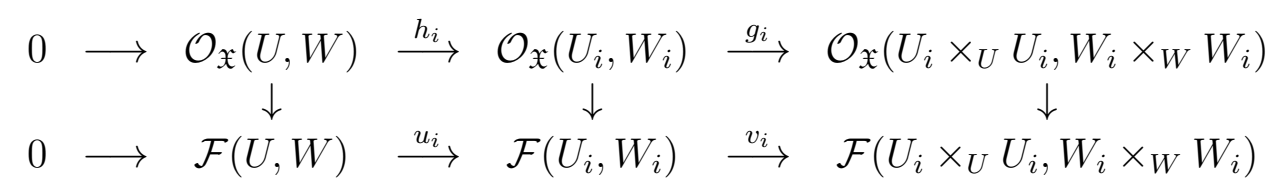

The arguments at b) above show that the map $g_{i}=0$ therefore the image of the natural map $\mathcal{O}_{\mathfrak{X}}\left(U_{i}, W_{i}\right) \longrightarrow \mathcal{F}\left(U_{i}, W_{i}\right)$ is contained in the image of $u_{i}$. More precisely the natural map: $\varphi: \mathcal{O}_{\mathfrak{X}}(U, W) \longrightarrow \mathcal{F}(U, W)$ has the property that $\varphi=w_{i} \circ h_{i}$ for $i=1$, 2, where $w_{i}: \mathcal{O}_{\mathfrak{X}}\left(U_{i}, W_{i}\right) \longrightarrow \mathcal{F}(U, W)$ is the map defined by the above diagram.

We remark that $\mathcal{O}_{\mathfrak{X}}(U, W)=B$ as $B$ is integral over $A$ and being smooth it is normal, similarly $\mathcal{O}_{\mathfrak{X}}\left(U_{i}, W_{i}\right)=B_{i}$, for $i=1,2$. Moreover we have $h_{i}=f_{i}$ for $i=1,2$. As $\mathcal{F}$ is the sheaf associated to the presheaf $\mathcal{O}_{\mathfrak{X}}$ the natural map $\mathcal{O}_{\mathfrak{X}}(U, W) / p \mathcal{O}_{\mathfrak{X}}(U, W) \longrightarrow \mathcal{G}(U, W)$ is the composition $\mathcal{O}_{\mathfrak{X}}(U, W) / p \mathcal{O}_{\mathfrak{X}}(U, W) \longrightarrow \mathcal{F}(U, W) / p \mathcal{F}(U, W) \longrightarrow \mathcal{G}(U, W)$. But the map

$$
\bar{\varphi}: \mathcal{O}_{\mathfrak{X}}(U, W) / p \mathcal{O}_{\mathfrak{X}}(U, W) \cong \bar{B} \cong \bar{B}_{1} \times \bar{B}_{2} \longrightarrow \mathcal{F}(U, W) / p \mathcal{F}(U, W)
$$

induced by $\varphi$ has the property that it factors through $\bar{f}_{i}$, for $i=1,2$ and $\bar{f}_{i}: \bar{B} \longrightarrow \bar{B}_{i}$ is the natural projection on the $i-t h$ factor. We deduce that $\bar{\varphi}=0$.

Faltings' site $\mathrm{PT}_{X_{M}}$. Let $X$ be a scheme of finite type over $\mathcal{O}_{K}$ and let $M$ be an algebraic extension of $K$. We denote by $E_{X_{M}}$ the category defined in definition 2.1.

Definition 2.3. Let $\left\{\left(U_{i}, W_{i}\right) \longrightarrow(U, W)\right\}_{i \in I}$ be a family of morphisms in $E_{X_{M}}$. We say that it is of type $\alpha$ respectively $\beta$ if:

$\alpha)\left\{U_{i} \longrightarrow U\right\}_{i \in I}$ is a covering in $X^{\text {et }}$ and $W_{i} \cong W \times_{U} U_{i}$ for every $i \in I$. Here the morphism $W \longrightarrow U$ used in the fibre product is the composition $W \longrightarrow U_{M} \longrightarrow U$.

or

B) $U_{i} \cong U$ for all $i \in I$ and $\left\{W_{i} \longrightarrow W\right\}_{i \in I}$ is a covering in $X_{M}^{\mathrm{fet}}$.

We endow $E_{X_{M}}$ with the topology $\mathrm{T}_{X_{M}}$ generated by the families of type $\alpha$ and $\beta$ described in definition 2.3 and denote by $\mathfrak{X}_{M}$ the associated site. We call $\mathrm{T}_{X_{M}}$ Faltings' topology and $\mathfrak{X}_{M}$ Faltings' site associated to $(X, M)$. Note that $\mathrm{T}_{X_{M}}$ can be described differently as follows.

Definition 2.4. A family $\left\{\left(U_{i j}, W_{i j}\right) \longrightarrow(U, W)\right\}_{i \in I, j \in J}$ of morphisms in $E_{X_{M}}$ is called a strict covering family if

a) For each $i \in I$ there exists an étale morphism $U_{i} \longrightarrow X$ such that we have isomorphisms $U_{i} \cong U_{i j}$ over $X$ for every $j \in J$.

b) $\left\{U_{i} \longrightarrow U\right\}_{i \in I}$ is a covering in $X^{\mathrm{et}}$.

c) For every $i \in I$ the family $\left\{W_{i j} \longrightarrow W \times_{U} U_{i}\right\}_{j \in J}$ is a covering in $X_{M}^{\mathrm{fet}}$.

To simplify notations we will henceforth denote a strict covering family $\left\{\left(U_{i j}, W_{i j}\right) \longrightarrow\right.$ $(U, W)\}_{i \in I, j \in J}$ by $\left\{\left(U_{i}, W_{i j}\right) \longrightarrow(U, W)\right\}_{i \in I, j \in J}$. 
Remark 2.5. The families of type $\alpha$ and $\beta$ in definition 2.3 are examples of strict coverings. Conversely a strict covering family $\left\{\left(U_{i}, W_{i j}\right) \longrightarrow(U, W)\right\}_{i \in I, j \in J}$ can be obtained as a composite of the covering $\left\{\left(U_{i}, W \times_{U}, U_{i}\right) \longrightarrow(U, W)\right\}_{i \in I, j \in J}$, which is of type $\left.\alpha\right)$ and for every $i \in I$ the covering $\left\{\left(U_{i}, W_{i j}\right) \longrightarrow\left(U_{i}, W \times_{U} U_{i}\right)\right\}_{j \in J}$, which is of type $\left.\beta\right)$. In particular the topology generated by the strict coverings coincides with $\mathrm{T}_{X_{M}}$.

Remark 2.6. The morphisms $\left(U_{i}, W_{i}\right) \longrightarrow(U, W)$, for $i=1,2$ in example 2.2 are not coverings in the sense of 2.3. In fact, it follows from 2.11 and 2.2 Faltings' topology associated to $(X, \bar{K})$ is coarser than the one originally introduced by Faltings.

Remark 2.7. The category $E_{X_{M}}$ with the strict covering families do not form a pre-topology. Indeed, since finite projective limits exist in $E_{X_{M}}$ the strict covering families satisfy PT0, PT1 and PT3 of [SGAIV, Def II 1.3] but contrary to what was written in [AI] and as was pointed out to us by A. Abbes, they do not satisfy PT2. However, one may define tautologically the generated pre-topology $\mathrm{PT}_{X_{M}}$ by considering as covering families the composite of finitely many strict coverings (or of finitely many families of type $\alpha$ ) and $\beta$ ) of definition 2.3). The associated topology is $\mathrm{T}_{X_{M}}$.

Remark 2.8. It follows from [SGAIV, Cor. II 2.3] or by a direct check using the definitions that a pre-sheaf on $E_{X_{M}}$ is a sheaf if and only if it satisfies the usual exactness property for the strict covering families.

The next lemma and [SGAIV, Remark II 3.3] show that it is enough to use strict covering families in order to sheafify a presheaf on $E_{X_{M}}$, as done in [AI].

Lemma 2.9. Let $(U, W)$ be an object of $E_{X_{M}}$. Then the strict covering families are cofinal in the collection of all covering families of $(U, W)$ in $\mathrm{PT}_{X_{M}}$.

Proof. See [Err].

Definition 2.10. We define the pre-sheaf of $\mathcal{O}_{M}$-algebras on $E_{X_{M}}$, denoted $\mathcal{O}_{\mathfrak{X}_{M}}$, by

$$
\mathcal{O}_{\mathfrak{X}_{M}}(U, W):=\text { the normalization of } \Gamma\left(U, \mathcal{O}_{U}\right) \text { in } \Gamma\left(W, \mathcal{O}_{W}\right) \text {. }
$$

We also define the sub pre-sheaf of $\mathcal{O}_{M_{0}}$-algebras $\mathcal{O}_{\mathfrak{X}_{M}}^{\text {un }}$ of $\mathcal{O}_{\mathfrak{X}_{M}}$ whose sections over $(U, W) \in E_{X_{M}}$ consist of elements $x \in \mathcal{O}_{\mathfrak{X}_{M}}(U, W)$ for which there exist a finite unramified extension $K \subset L$, a finite étale morphism $U^{\prime} \rightarrow U \otimes_{\mathcal{O}_{K}} \mathcal{O}_{L}$ and a morphism $W \rightarrow U_{K}^{\prime} \otimes_{L} M$ over $U_{M}$ such that $x$, viewed in $\Gamma\left(W, \mathcal{O}_{W}\right)$, lies in the image of $\Gamma\left(U^{\prime}, \mathcal{O}_{U^{\prime}}\right)$.

We have

Proposition 2.11. The pre-sheaves $\mathcal{O}_{\mathfrak{X}_{M}}$ and $\mathcal{O}_{\mathfrak{X}_{M}}^{\text {un }}$ are sheaves.

Proof. We first prove that $\mathcal{O}_{\mathfrak{X}_{M}}$ is a sheaf. Let $\left\{\left(U_{\alpha}, W_{\alpha, i}\right) \longrightarrow(U, W)\right\}_{\alpha, i}$ be a strict covering family. We set $U_{\alpha \beta}:=U_{\alpha} \times_{U} U_{\beta}$ and $W_{\alpha \beta i j}:=W_{\alpha, i} \times_{W} W_{\beta, j}$. We have the following commutative diagram

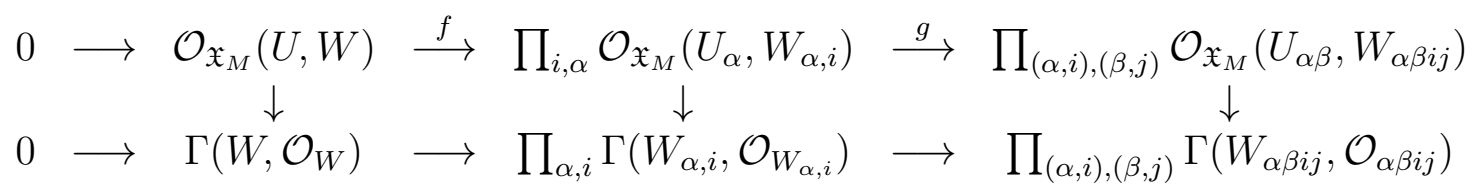


Since the $\left\{U_{\alpha} \longrightarrow U\right\}_{\alpha}$ is a covering in $X^{\text {et }}$ and for every $\alpha,\left\{W_{\alpha, i} \longrightarrow W \times_{U} U_{\alpha}\right\}_{i}$ is a covering in $\left(W \times_{U} U_{\alpha, M}\right)^{\mathrm{fet}}$ it follows that $\left\{W_{\alpha, i} \longrightarrow W\right\}_{\alpha, i}$ is a covering in $X_{M}^{\mathrm{et}}$. In particular the bottom row of the above diagram is exact. Moreover the vertical maps are all inclusions therefore $f$ is injective, i.e. $\mathcal{O}_{\mathfrak{X}_{M}}$ is a separable pre-sheaf. Let $x \in \operatorname{Ker}(g)$. Then $x \in \Gamma\left(W, \mathcal{O}_{W}\right) \cap$ $\prod_{\alpha, i} \mathcal{O}_{\mathfrak{X}_{M}}\left(U_{\alpha}, W_{\alpha, i}\right)$. We are left to prove that $x$ is integral over $\Gamma\left(U, \mathcal{O}_{U}\right)$. Without loss of generality we may assume that $W$ is connected and that $U_{\alpha}=\operatorname{Spec}\left(A_{\alpha}\right)$ is affine for every $\alpha$. Note that there exists a finite extension $K \subset L$ in $M$ and a finite and étale morphism $W^{\prime} \rightarrow U_{L}$ so that its base change via $L \rightarrow M$ is $W \rightarrow U_{M}$ and $x \in \Gamma\left(W^{\prime}, \mathcal{O}_{W^{\prime}}\right)$. Let us denote by $x_{\alpha}$ the image of $x$ in $\Gamma\left(W^{\prime} \times_{U} U_{\alpha}, \mathcal{O}_{W^{\prime} \times_{U} U_{\alpha}}\right)$. Because the family $\left\{W_{\alpha, i} \longrightarrow W \times_{U} U_{\alpha}\right\}_{i}$ is a covering family in $\left(W \times_{U} U_{\alpha, M}\right)^{\text {fet }}$ and the image $x_{\alpha, i}$ of $x_{\alpha}$ in $\Gamma\left(W_{\alpha, i}, \mathcal{O}_{W_{\alpha, i}}\right)$ is in fact in $\mathcal{O}_{\mathfrak{x}_{M}}\left(U_{\alpha}, W_{\alpha, i}\right)$, hence integral over $A_{\alpha}$, it follows that $x_{\alpha}$ is integral over $A_{\alpha}$. Let $P_{\alpha}(X) \in A_{\alpha}[X]$ be the (monic) characteristic polynomial of $x_{\alpha}$ over $A_{\alpha}$ with respect to the finite and étale extension $W^{\prime} \times_{U} U_{\alpha} \longrightarrow U_{\alpha, K}$ (see remark 2.12 below.) Then $\left.P_{\alpha}(X)\right|_{U_{\alpha \beta}}=\left.P_{\beta}(X)\right|_{U_{\alpha \beta}}$ for all $\alpha$ and $\beta$ and, therefore, there is a monic polynomial $P(X) \in \Gamma\left(U, \mathcal{O}_{U}\right)$ such that $\left.P(X)\right|_{U_{\alpha}}=P_{\alpha}(X)$. As $\left.P(x)\right|_{U_{\alpha}}=P_{\alpha}\left(x_{\alpha}\right)=0$ for every $\alpha$ it follows that $P(x)=0$, i.e. that $x$ is integral over $\Gamma\left(U, \mathcal{O}_{U}\right)$.

Since $\mathcal{O}_{\mathfrak{X}_{M}}^{\text {un }} \subset \mathcal{O}_{\mathfrak{X}_{M}}$ by construction, it follows that $\mathcal{O}_{\mathfrak{X}_{M}}^{\text {un }}$ is a separated pre-sheaf. Using the previous notations, it suffices to show that given $W$ connected and $U_{\alpha}$ 's affine and given $x \in \prod_{i, \alpha} \mathcal{O}_{\mathfrak{X}_{M}}^{\text {un }}\left(U_{\alpha}, W_{\alpha, i}\right)$, whose image in $\prod_{i, \alpha} \mathcal{O}_{\mathfrak{X}_{M}}\left(U_{\alpha}, W_{\alpha, i}\right)$ lies in $\operatorname{Ker}(g)$, then $x \in \mathcal{O}_{\mathfrak{X}_{M}}^{\text {un }}(U, W)$. As before for every $\alpha$ let $x_{\alpha}$ be the image of $x$ in $\Gamma\left(W_{\alpha}, \mathcal{O}_{W_{\alpha}}\right)$. By replacing the $U_{\alpha}$ 's by a finite subcover, we may assume that we have only finitely many $\alpha$ 's. By definition there is a finite unramified extension $K \subset L^{\prime}$ such that each $x_{\alpha}$ is defined over a finite and étale cover of $U_{\alpha} \otimes_{\mathcal{O}_{K}} \mathcal{O}_{L^{\prime}}$. Possibly after enlarging $L$, we may assume that $L^{\prime} \subset L$. Let $W_{\alpha}^{\prime}$ (resp. $Z_{\alpha}^{\prime}$ ) be the spectrum of the normalization of the sub-algebra $A_{\alpha} \otimes_{\mathcal{O}_{K}} L\left[x_{\alpha}\right]$ (resp. $A_{\alpha} \otimes_{\mathcal{O}_{K}} \mathcal{O}_{L^{\prime}}\left[x_{\alpha}\right]$ ) in $\Gamma\left(W \times_{U} U_{\alpha}, \mathcal{O}_{W \times_{U} U_{\alpha}}\right)$. By construction $Z_{\alpha}^{\prime}$ is finite and étale over $U \otimes_{\mathcal{O}_{K}} \mathcal{O}_{L}$ and we have morphisms $W_{\alpha}^{\prime} \rightarrow Z_{\alpha, K}^{\prime} \otimes_{L^{\prime}} L$ over $U_{\alpha, L}$. Moreover, $x_{\alpha} \in \Gamma\left(W_{\alpha}^{\prime}, \mathcal{O}_{W_{\alpha}^{\prime}}\right)$ is in the image of $\Gamma\left(Z_{\alpha}^{\prime}, \mathcal{O}_{Z_{\alpha}^{\prime}}\right)$. Note that $W_{\alpha}^{\prime} \times_{U_{\alpha}} U_{\alpha \beta} \cong W_{\beta}^{\prime} \times_{U_{\beta}} U_{\alpha \beta}$ so that the various $W_{\alpha}^{\prime}$ glue to a finite and étale morphism $W^{\prime} \rightarrow U_{L}$ and there is a morphism $W \rightarrow W^{\prime}$ as schemes over $U_{L}$ such that $x \in \Gamma\left(W^{\prime}, \mathcal{O}_{W^{\prime}}\right)$. Moreover, also the various $Z_{\alpha}^{\prime}$ glue to a scheme $Z^{\prime}$ finite and étale over $U \otimes_{\mathcal{O}_{K}} \mathcal{O}_{L^{\prime}}$ and we have a morphism $W^{\prime} \rightarrow Z^{\prime} \otimes_{\mathcal{O}_{L^{\prime}}} L$. Then $x$ is in the image of $\Gamma\left(Z^{\prime}, \mathcal{O}_{Z^{\prime}}\right)$ and we conclude that $x \in \mathcal{O}_{\mathfrak{X}_{M}}^{\text {un }}(U, W)$ as claimed.

The following argument was offered by the referee of the paper.

Remark 2.12. Let $A$ be a noetherian normal domain and let $B$ be the integral closure of $A$ in a finite étale extension of $A[1 / p]$. Let $x \in B[1 / p]$ be an element and let $Q(X) \in A[1 / p][X]$ be the characteristic polynomial of $x$ (it exists as $B[1 / p]$ is a finitely generated projective $A[1 / p]$ module.) Then $x \in B$ if and only if $Q(X) \in A[X]$.

Proof. The sufficiency is clear and to prove necessity, as $A$ is a noetherian normal domain, it is enough to prove that $Q(X) \in A_{\mathfrak{p}}$, for all $\mathfrak{p}$ prime ideal of height 1 of $A$ which contains $p$. Hence we may assume that $A$ is a DVR and in this case $B$ is a free $A$-module of finite rank and $Q(X)$ is the characteristic polynomial of the matrix associated to the endomorphism $B \longrightarrow B ; b \rightarrow x b$ with respect to a basis of $B$ over $A$. Therefore $Q(X) \in A[X]$. 


\subsection{Faltings' topos; the formal setting}

Let now $X$ denote a formal scheme. Denote by $X^{\text {et }}$ the étale site on $X$ and by $\operatorname{Sh}\left(X^{\mathrm{et}}\right)$ the category of sheaves of abelian groups on $X^{\mathrm{et}}$. Of particular importance will be the so called small affine opens of $X^{\text {et }}$. These are objects $\mathcal{U}$ such that $\mathcal{U}=\operatorname{Spf}\left(R_{\mathcal{U}}\right)$ is affine and connected and there are parameters $T_{1}, T_{2}, \ldots, T_{d} \in R_{\mathcal{U}}^{\times}$such that the map $R_{0}:=\mathcal{O}_{K}\left\{T_{1}^{ \pm 1}, \ldots, T_{d}^{ \pm 1}\right\} \subset R_{\mathcal{U}}$ is formally étale.

The site $X_{M \text {,et }}$. For every finite extension $K \subset L$ in $M$ let $X_{L \text {,et }}$ be the site of étale and quasi-compact morphisms $\mathcal{W} \rightarrow X_{L}$ of $L$-rigid analytic spaces. Here $X_{K}$ denotes the $K$-rigid analytic space associated to $X$ and $X_{L}$ is its base change to $L$. We refer to [JvdP, $\S 3.1 \& 3.2$ ] for generalities about étale morphisms of rigid analytic spaces. Given extensions $L \subset L^{\prime}$ of $K$ contained in $M$ the base change from $L$ to $L^{\prime}$ provides a morphism of sites $X_{L, \text { et }} \rightarrow X_{L^{\prime} \text {,et }}$. We then get a fibred site $X_{*, \text { et }}$ over the category of finite extensions of $K$ contained in $M$ in the sense of [SGAIV, $§$ VI.7.2.1]. We let $X_{M \text {,et }}$ be the site defined by the projective limit of the fibred site $X_{*, \text { et }}$; see [SGAIV, Def. VI.8.2.5].

We can give the following explicit description. The objects in $X_{M \text {,et }}$ consist of pairs $(\mathcal{W}, L)$ where $L$ is a finite extension of $K$ contained in $M$ and $\mathcal{W} \rightarrow X \otimes_{K} L$ is an étale and quasi-compact map of $L$-rigid analytic spaces. Given $(\mathcal{W}, L)$ and $\left(\mathcal{W}^{\prime}, L^{\prime}\right)$ define $\operatorname{Hom}_{X_{M, \text { et }}}\left(\left(\mathcal{W}^{\prime}, L^{\prime}\right),(\mathcal{W}, L)\right)$ as the direct limit $\lim _{\rightarrow} \operatorname{Hom}_{L^{\prime \prime}}\left(\mathcal{W}^{\prime} \otimes_{L^{\prime}} L^{\prime \prime}, \mathcal{W} \otimes_{L} L^{\prime \prime}\right)$ over all finite extensions $L^{\prime \prime} \subset M$, containing both $L$ and $L^{\prime}$, of the morphism $\mathcal{W}^{\prime} \otimes_{L^{\prime}} L^{\prime \prime} \rightarrow \mathcal{W} \otimes_{L} L^{\prime \prime}$ as rigid analytic spaces over $X \otimes_{L} L^{\prime \prime}$. The coverings of a pair $(\mathcal{W}, L)$ in $X_{M \text {,et }}$ are finite families of pairs $\left\{\left(\mathcal{W}_{\alpha}, L_{\alpha}\right)\right\}_{\alpha}$ over $(\mathcal{W}, L)$ such that $L \subset L_{\alpha}$ for every $\alpha$ and there exists a finite extension of $K$, contained in $M$ and containing $L_{\alpha}$ for every $\alpha$ such that the induced map $\amalg_{\alpha} \mathcal{W}_{\alpha} \otimes_{L_{\alpha}} L^{\prime} \rightarrow \mathcal{W} \otimes_{L} L^{\prime}$ is surjective.

The site $\mathcal{U}_{M \text {,fet }}$ Let $\mathcal{U} \rightarrow X$ be an étale map topologically of finite type of $p$-adic formal schemes. Define $\mathcal{U}^{* \text {,fet }}$ to be the following site fibred over the category of finite extensions $K \subset L$ contained in $M$. For very such $L$ write $\mathcal{U}^{L \text {,fet }}$ to be the category of finite étale covers $\mathcal{W} \rightarrow \mathcal{U}_{L}$ as $L$-rigid analytic spaces. Given extensions $L \rightarrow L^{\prime}$ we consider the base change map $\mathcal{U}^{L \text {,fet }} \rightarrow \mathcal{U}^{L^{\prime} \text {,fet }}$. Define $\mathcal{U}_{\mathrm{M} \text {,fet }}$ as the projective limit site. For notational purposes we write $(\mathcal{W}, L)$, or simply $\mathcal{W}$, for an object of $\mathcal{U}_{M \text {,fet. }}$. In the first notation we implicitly assume that $\mathcal{W} \in \mathcal{U}^{L \text {,fet }}$. We refer to [AI, §4.1] for an explicit description. Note that the fiber product of two pairs over a given one exists in $\mathcal{U}_{M \text {,fet }}$ and, if those are defined in $\mathcal{U}_{L \text {,fet }}$ for some $L$, it coincides with the image of the fibre product in $\mathcal{U}_{L \text {,fet. }}$.

Let $\mathcal{U}_{2} \rightarrow \mathcal{U}_{1}$ be a map of formal schemes over $X$. Assume that they are étale over $X$. We then have a functor $\mathcal{U}_{1, \text {, fet }} \rightarrow \mathcal{U}_{2, \text {, fet }}$ of sites fibred over the category of finite extensions $K \subset L$ contained in $M$. We let

$$
\rho_{\mathcal{U}_{1}, \mathcal{U}_{2}}: \mathcal{U}_{1, M, \text { fet }} \rightarrow \mathcal{U}_{2, M, \text { fet }}
$$

be the induced morphisms of projective limits. It is given on objects by

$$
(\mathcal{W}, L) \mapsto\left(\mathcal{W} \times_{\mathcal{U}_{1, K}} \mathcal{U}_{2, K}, L\right)
$$

The category $E_{X_{M}}$ and Faltings' topology $\mathrm{T}_{X_{M}}$. Define $E_{X_{M}}$ to be the category of pairs $(\mathcal{U}, \mathcal{W})$ where $\mathcal{U} \rightarrow X$ is an étale map of formal schemes and $\mathcal{W}$ is an object of $\mathcal{U}_{\mathrm{M} \text {,fet. A morphism of }}$ 
pairs $\left(\mathcal{U}^{\prime}, \mathcal{W}^{\prime}\right) \rightarrow(\mathcal{U}, \mathcal{W})$ is defined to be a morphism $\mathcal{U}^{\prime} \rightarrow \mathcal{U}$ as formal schemes over $X$ and a $\operatorname{map} \mathcal{W}^{\prime} \rightarrow \mathcal{W} \times{ }_{\mathcal{U}_{K}} \mathcal{U}_{K}^{\prime}$ in $\mathcal{U}_{\mathrm{M}, \text { fet }}^{\prime}$.

We define strict covering families exactly as in definition 2.4 and Faltings' topology $\mathrm{T}_{X_{M}}$ to be the topology generated by the strict covering families. We call the associated site the locally Galois site attached to the data $(X, M)$ and denote it by $\mathfrak{X}_{M}$.

We define the pre-sheaves $\mathcal{O}_{\mathfrak{X}_{M}}$ and $\mathcal{O}_{\mathfrak{X}_{M}}^{\text {un }}$ on $E_{X_{M}}$ as in definition 2.10, The analogue of proposition 2.11 holds in our formal context i.e., $\mathcal{O}_{\mathfrak{X}_{M}}$ and $\mathcal{O}_{\mathfrak{X}_{M}}^{\text {un }}$ are sheaves.

\subsection{Continuous functors. Localization functors}

We define:

I.a if $X$ is a scheme over $\mathcal{O}_{K}$, we have $u_{X, M}: \mathfrak{X}_{M} \longrightarrow X_{M, \text { et }}$ with $u_{X, M}(U, W):=W$;

I.b if $X$ is a $p$-adic formal scheme over $\mathcal{O}_{K}$, let $u_{X, M}: \mathfrak{X}_{M} \longrightarrow X_{M, \text { et }}$ be $u_{X, M}(\mathcal{U},(\mathcal{W}, L)):=$ $(\mathcal{W}, L)$

II.a if $X$ is a scheme of finite type over $\mathcal{O}_{K}$, let $v_{X, M}: X_{\text {et }} \longrightarrow \mathfrak{X}_{M}$ be given by $v_{X, M}(U):=$ $\left(U, U_{M}\right)$

II.b if $X$ is a formal scheme locally topologically of finite type over $\mathcal{O}_{K}$, we have $v_{X, M}: X_{\text {et }} \longrightarrow$ $\mathfrak{X}_{M}$ given by $v_{X, M}(\mathcal{U}):=\left(\mathcal{U}, \mathcal{U}_{K}\right)$;

Let $K \subset M_{1} \subset M_{2} \subset \bar{K}$ be field extensions. Define

III $\beta_{M_{1}, M_{2}}: \mathfrak{X}_{M_{1}} \rightarrow \mathfrak{X}_{M_{2}}$ by $\beta_{M_{1}, M_{2}}(U, W)=\left(U, W \otimes_{M_{1}} M_{1}\right)$ (resp. $\beta_{M_{1}, M_{2}}(\mathcal{U}, \mathcal{W})$ equal to $(\mathcal{U}, \mathcal{W})$ viewed in $\mathfrak{X}_{M_{2}}$ ) in the algebraic (resp. formal) setting.

It is clear that the above functors send covering families to covering families and commute with fiber products. In particular they define continuous functors of sites by [SGAIV, Prop. III.1.6]. They also send final objects to final objects so that they induce morphisms of the associated topoi of sheaves.

Following [Err] we define a geometric point of $\mathfrak{X}$ to be a pair $(x, y)$ where $x$ is a geometric point of $X$ and $y$ is a geometric point of $X_{K}$ specializing to $x$ i.e., a geometric point of the henselization of $X$ at $x$. In loc. cit., we define the stalk $\mathcal{F}_{(x, y)}$ of a sheaf $\mathcal{F}$ on $\mathfrak{X}$ to be the direct $\operatorname{limit} \lim \mathcal{F}(U, W)$ over all pairs $\left(\left(U, x^{\prime}\right),\left(W, y^{\prime}\right)\right)$ where $x^{\prime}$ is a point of $U$ mapping to $x$ and $y^{\prime}$ is a point of $W$ specializing to $x^{\prime}$ and mapping to $y$. We proved in loc. cit. that there are enough geometric point in $\mathfrak{X}$ i.e., that a sequence of sheaves is exact if an only if the induced sequence on stalks is exact for all geometric points.

Lemma 2.13. Both in the algebraic and in the formal setting we have an isomorphism of sheaves $v_{X, M}^{*}\left(\mathcal{O}_{X}\right) \cong \mathcal{O}_{\mathfrak{X}_{M}}^{\text {un }}$ on $\mathfrak{X}_{M}$.

Proof. Let $Q$ be the pre-sheaf on $\mathfrak{X}$ defined by $Q(U, W):=\Gamma\left(U, \mathcal{O}_{U}\right)$ if $W \neq \phi$ and $Q(U, \phi)=0$. It is a separated pre-sheaf. Note that if $W \neq \phi,\left(U, U_{K}\right)$ is the initial object in the category of all pairs $\left(U^{\prime}, W^{\prime}\right)$ admitting a morphism $(U, W) \rightarrow\left(U^{\prime}, W^{\prime}\right)$ in $\mathfrak{X}$. Thus, $v_{X, M}^{*}\left(\mathcal{O}_{X}\right)$ is the sheaf on $\mathfrak{X}$ associated to the pre-sheaf $Q$. Note also that we have a natural map $Q \rightarrow \mathcal{O}_{\mathfrak{X}_{M}}^{\text {un }}$. Let 
$a \in \mathcal{O}_{\mathfrak{X}_{M}}^{\text {un }}(U, W)$ and view it in $\Gamma\left(W, \mathcal{O}_{W}\right)$. By definition there exists a finite extension $K \subset L$ in $M$ and a finite and étale morphism $U^{\prime} \longrightarrow U \otimes_{\mathcal{O}_{K}} \mathcal{O}_{L}$ so that we have a map $W \rightarrow U_{K}^{\prime} \otimes_{L} M$ over $U_{M}$ and $a$ is in the image of $\Gamma\left(U^{\prime}, \mathcal{O}_{U^{\prime}}\right)$ in $\Gamma\left(W, \mathcal{O}_{W}\right)$. Note that $U^{\prime}$ is a direct factor of $U^{\prime} \otimes_{\mathcal{O}_{K}} \mathcal{O}_{L}$ so that $a$ is in the image of $Q\left(U^{\prime}, W\right)$ in $\Gamma\left(W, \mathcal{O}_{W}\right)$ as wanted. This proves that the natural morphism $Q \longrightarrow \mathcal{O}_{\mathfrak{X}_{M}}^{\text {un }}$ is surjective. To prove injectivity let $(U, W)$ be such that $U$ is connected and $W \neq \phi$. Since the composition

$$
Q(U, W) \longrightarrow \mathcal{O}_{\mathfrak{X}_{M}}^{\text {un }}(U, W) \subset \Gamma\left(W, \mathcal{O}_{W}\right)
$$

is injective we deduce that the first map is injective. It follows that the induced morphism from the sheaf associated to $Q$ to $\mathcal{O}_{\mathfrak{X}_{M}}^{\text {un }}$ is injective.

The localization functors. For this section we suppose that $X$ is either a smooth scheme or a smooth formal scheme over $\mathcal{O}_{K}$. Let $\mathcal{U}$ be a connected affine open in the étale site of $X$ with underlying algebra $R_{\mathcal{U}}$. Write $R_{\mathcal{U}} \otimes_{\mathcal{O}_{K}} M:=\prod_{i=1}^{n} R_{\mathcal{U}, i}$ with $\operatorname{Spec}\left(R_{\mathcal{U}, i}\right)$ connected. Fix a geometric generic point $\bar{\eta}_{i}=\operatorname{Spec}\left(\mathbb{C}_{\mathcal{U}, i}\right)$ of $\operatorname{Spec}\left(R_{\mathcal{U}, i}\right)$ and denote by $\bar{R}_{\mathcal{U}, i}$ the union of all finite normal $R_{\mathcal{U}}$ sub-algebras of $\mathbb{C}_{\mathcal{U}, i}$, which are finite and étale over $R_{\mathcal{U}, i}$ after inverting $p$. We let $\mathcal{G}_{\mathcal{U}_{M}, i}$ be the Galois group of $R_{\mathcal{U}, i} \subset \bar{R}_{\mathcal{U}_{, i}} \otimes_{\mathcal{O}_{K}} K$. Eventually, let $\bar{R}_{\mathcal{U}}:=\prod_{i=1}^{n} \bar{R}_{\mathcal{U}, i}$ and let

$$
\mathcal{G}_{\mathcal{U}_{M}}:=\prod_{i=1}^{n} \mathcal{G}_{\mathcal{U}_{M}, i} .
$$

Let $\operatorname{Rep}\left(\mathcal{G}_{\mathcal{U}_{M}}\right)$ (resp. $\left.\operatorname{Rep}\left(\mathcal{G}_{\mathcal{U}_{M}}\right)^{\mathbb{N}}\right)$ be the category of discrete abelian groups (resp. the category of inverse systems of finite abelian groups indexed by $\mathbb{N}$ ) with continuous action of $\mathcal{G}_{\mathcal{U}_{M}}$. We have natural functors, which we'll call localization functors

$$
\operatorname{Sh}\left(\mathfrak{X}_{M}\right) \longrightarrow \operatorname{Rep}\left(\mathcal{G}_{\mathcal{U}_{M}}\right) \quad \text { and } \quad \operatorname{Sh}\left(\mathfrak{X}_{M}\right)^{\mathbb{N}} \longrightarrow \operatorname{Rep}\left(\mathcal{G}_{\mathcal{U}_{M}}\right)^{\mathbb{N}}
$$

defined as follows (we only define the functor in the case $X$ is a scheme over $\mathcal{O}_{K}$ as above and leave it to the reader to fill in the details for the other cases): if $\mathcal{G} \in \operatorname{Sh}\left(\mathfrak{X}_{M}\right)$ is a sheaf of abelian groups, its localization is $\mathcal{G}\left(\bar{R}_{\mathcal{U}}\right):=\oplus_{i=1}^{n} \mathcal{G}\left(\bar{R}_{\mathcal{U}, i}\right)$ where $\mathcal{G}\left(\bar{R}_{\mathcal{U}, i}\right):=\lim _{\rightarrow} \mathcal{G}(\mathcal{U}, \operatorname{Spec}(S))$, for $S$ running over all $R_{\mathcal{U}, i}$ sub-algebras of $\bar{R}_{\mathcal{U}, i} \otimes_{\mathcal{O}_{K}} K$ which are finite and étale. It is a set with the discrete topology and it is endowed with a continuous action of $\mathcal{G}_{\mathcal{U}_{M}}$. The objects $(\mathcal{U}, \mathcal{W})$ of $\mathfrak{X}_{M}$, with $\mathcal{W}=\operatorname{Spec}(S)$ and $R_{\mathcal{U}, i} \rightarrow S \subset \bar{R}_{\mathcal{U}_{, i}} \otimes_{\mathcal{O}_{K}} K$, correspond to finite index sub-groups $G_{\mathcal{W}} \subset \mathcal{G}_{\mathcal{U}_{M}, i}$. For any such, we can recover $\mathcal{G}(\mathcal{U}, \mathcal{W})$ from the localization of $\mathcal{G}$ by the formula $\mathcal{G}(\mathcal{U}, \mathcal{W})=\mathcal{G}\left(\bar{R}_{\mathcal{U}, i}\right)^{G_{\mathcal{W}}}$. This allows to recover $\mathcal{G}(\mathcal{U}, \mathcal{W})$ for every $\mathcal{W} \rightarrow \mathcal{U}_{M}$ finite and étale (see [AI, Lemma 4.5.3]).

Lemma 2.14. Let $\mathcal{G}$ be a sheaf on $\mathfrak{X}_{M_{1}}$. Let $M_{1} \subset M_{2}$ be a Galois field extension. Then

i. the sheaf $\beta_{M_{1}, M_{2}}^{*}(\mathcal{G})$ coincides with the pre-sheaf $\beta_{M_{1}, M_{2}}^{-1}(\mathcal{G})$;

ii. for every object $(\mathcal{U}, \mathcal{W})$ of $\mathfrak{X}_{M_{1}}$ the group $\beta_{M_{1}, M_{2}, *}\left(\beta_{M_{1}, M_{2}}^{*}(\mathcal{G})\right)(\mathcal{U}, \mathcal{W})$ is endowed with an action of $\operatorname{Gal}\left(M_{2} / M_{1}\right)$ and $\mathcal{G}(\mathcal{U}, \mathcal{W})=\left(\beta_{M_{1}, M_{2}, *}\left(\beta_{M_{1}, M_{2}}^{*}(\mathcal{G})\right)(\mathcal{U}, \mathcal{W})\right)^{\operatorname{Gal}\left(M_{2} / M_{1}\right)}$. 
iii. take $\mathcal{U}=\operatorname{Spf}\left(R_{\mathcal{U}}\right)$ to be a connected affine open in the étale site of $X$. Then

$$
\beta_{M_{1}, M_{2}, *}\left(\beta_{M_{1}, M_{2}}^{*}(\mathcal{G})\right)\left(\bar{R}_{\mathcal{U}}\right) \cong \mathcal{G}\left(\bar{R}_{\mathcal{U}}\right)^{\left[M_{2}: M_{1}\right]}
$$

Proof. We prove the statements in the formal case, leaving the algebraic case to the reader.

(i) Given an object $(\mathcal{U}, \mathcal{W})$ of $\mathfrak{X}_{M_{2}}$, the group $\beta_{M_{1}, M_{2}}^{-1}(\mathcal{G})(\mathcal{U}, \mathcal{W})$ is $\lim _{\mathcal{W}^{\prime}} \mathcal{G}\left(\mathcal{U}, \mathcal{W}^{\prime}\right)$ where the direct limit is taken over all objects $\left(\mathcal{U}, \mathcal{W}^{\prime}\right) \in \mathfrak{X}_{M_{1}}$ and all morphisms from $(\mathcal{U}, \mathcal{W})$ to $\left(\mathcal{U}, \mathcal{W}^{\prime}\right)$ in $\mathfrak{X}_{M_{2}}$. Note that $\mathcal{W}$ is finite and étale over $\mathcal{U}_{L}$ for some finite extension $K \subset L$ contained in $M_{2}$. In particular it is a finite and étale over $\mathcal{U}_{L^{\prime}}$ for $L^{\prime}:=M_{1} \cap L$. Thus the direct limit admits as final object the group $\mathcal{G}(\mathcal{U}, \mathcal{W})$ with $(\mathcal{U}, \mathcal{W})$ viewed as an object of $\mathfrak{X}_{M_{1}}$. The first claim follows; see also [AI, Pf. Prop. 4.4.2(4)].

(ii) Take an object $(\mathcal{U}, \mathcal{W})$ of $\mathfrak{X}_{M_{1}}$. Assume that $\mathcal{W}$ is finite and étale over $\mathcal{U}_{L}$ with $L \subset$ $M_{1}$. Then $\beta_{M_{1}, M_{2}, *}\left(\beta_{M_{1}, M_{2}}^{*}(\mathcal{G})\right)(\mathcal{U}, \mathcal{W})$ coincides with the direct limit $\mathcal{G}\left(\mathcal{U}, \mathcal{W}_{L^{\prime}}\right)$ over all finite extensions $L \subset L^{\prime} \subset M_{2}$ where $\mathcal{W}_{L^{\prime}}$ is considered as an étale covering of $\mathcal{U}_{L^{\prime \prime}}$ for $L^{\prime \prime}:=L^{\prime} \cap M_{1}$. The Galois group $\operatorname{Gal}\left(M_{2} / M_{1}\right)$ acts on this set and the invariants under $\operatorname{Gal}\left(L^{\prime} M_{1} / M_{1}\right)$ are exactly $\mathcal{G}(\mathcal{U}, \mathcal{W})$. The claim follows.

(iii) It follows from (ii) and the fact that $\bar{R}_{\mathcal{U}}\left[p^{-1}\right] \otimes_{M_{1}} M_{2}=\bar{R}_{\mathcal{U}}\left[p^{-1}\right]^{\left[M_{2}: M_{1}\right]}$.

\subsection{The sheaf $\mathbb{A}_{\text {inf,M }}^{+}$.}

Let us recall the following definitions from $\S 5$ of $[\mathrm{AI}]$. Denote by $\widehat{\mathcal{O}}_{\mathfrak{x}_{M}}$ the inverse system of sheaves of $\mathcal{O}_{M}$-algebras $\left\{\mathcal{O}_{\mathfrak{X}_{M}} / p^{n} \mathcal{O}_{\mathfrak{X}_{M}}\right\}_{n} \in \operatorname{Sh}\left(\mathfrak{X}_{M}\right)^{\mathbb{N}}$.

For every $s \in \mathbb{N}$ define $\mathbb{W}_{s, M}:=\mathbb{W}_{s}\left(\mathcal{O}_{\mathfrak{x}_{M}} / p \mathcal{O}_{\mathfrak{x}_{M}}\right)$; it is the sheaf $\left(\mathcal{O}_{\mathfrak{X}_{M}} / p \mathcal{O}_{\mathfrak{X}_{M}}\right)^{s}$ with ring operations defined by Witt polynomials and the transition maps in the inverse system defined by Frobenius. Let $\mathbb{A}_{\text {inf,M }}^{+}$in $\operatorname{Sh}\left(\mathfrak{X}_{M}\right)^{\mathbb{N}}$ be the inverse system of sheaves of $\mathbb{W}(k)$-algebras $\left\{\mathbb{W}_{n, M}\right\}_{n}$ where the transition maps are defined as the composite of the natural projection $\mathbb{W}_{n+1, M} \rightarrow$ $\mathbb{W}_{n, M}$ and Frobenius on $\mathbb{W}_{n, M}$. Note that $\mathbb{A}_{\text {inf,M }}^{+}$is endowed with a Frobenius operator, denoted by $\varphi$, and is a sheaf of $\mathcal{O}_{M_{0}}$-algebras.

If $M_{1} \subset M_{2}$ is a field extension, it follows from 2.14 that we have a natural isomorphism $\beta_{M_{1}, M_{2}}^{*}\left(\mathcal{O}_{\mathfrak{X}_{M_{1}}}\right) \cong \mathcal{O}_{\mathfrak{X}_{M_{2}}}$. In particular we have a natural map

$$
\beta_{M_{1}, M_{2}}^{*}\left(\mathbb{W}_{s, M_{1}}\right) \longrightarrow \mathbb{W}_{s, M_{2}}
$$

which is an isomorphism since $\beta_{M_{1}, M_{2}}^{*}$ is exact. In particular we have natural isomorphisms of inverse systems of sheaves $\beta_{M_{1}, M_{2}}^{*}\left(\widehat{\mathcal{O}}_{\mathfrak{X}_{M_{1}}}\right) \cong \widehat{\mathcal{O}}_{\mathfrak{X}_{M_{2}}}$ and $\beta_{M_{1}, M_{2}}^{*}\left(\mathbb{A}_{\text {inf, } M_{1}}^{+}\right) \cong \mathbb{A}_{\text {inf, } \mathrm{M}_{2}}^{+}$.

Proposition 2.15. Let $\mathcal{U}$ be a small affine object of the site $X^{\mathrm{et}}$; see \2.2 for the definition. Then the natural maps

a) $\widehat{\bar{R}}_{\mathcal{U}} \longrightarrow \lim _{\infty \leftarrow n}\left(\mathcal{O}_{\mathfrak{X}_{M}} / p^{n} \mathcal{O}_{\mathfrak{X}_{M}}\right)\left(\bar{R}_{\mathcal{U}}\right)=: \widehat{\mathcal{O}}_{\mathfrak{X}_{M}}\left(\bar{R}_{\mathcal{U}}\right)$,

b) $A_{\text {inf }}^{+}\left(\bar{R}_{\mathcal{U}}\right) \longrightarrow \lim _{\infty \leftarrow n} \mathbb{W}_{n, M}\left(\bar{R}_{\mathcal{U}}\right)=: \mathbb{A}_{\mathrm{inf}, \mathrm{M}}^{+}\left(\bar{R}_{\mathcal{U}}\right)$.

are isomorphisms. 
We'll only prove the result in the formal case and leave it to the reader to repeat the arguments in the algebraic case. First of all we prove

Lemma 2.16. For every $n \in \mathbb{N}$ the pre-sheaf $\mathcal{O}_{\mathfrak{x}_{M}} / p^{n} \mathcal{O}_{\mathfrak{X}_{M}}$ is separated $i$. e., if $\left(\mathcal{U}^{\prime}, \mathcal{W}^{\prime}\right) \rightarrow$ $(\mathcal{U}, \mathcal{W})$ is a covering, the natural map

$$
\mathcal{O}_{\mathfrak{x}_{M}}(\mathcal{U}, \mathcal{W}) / p^{n} \mathcal{O}_{\mathfrak{X}_{M}}(\mathcal{U}, \mathcal{W}) \longrightarrow \mathcal{O}_{\mathfrak{x}_{M}}\left(\mathcal{U}^{\prime}, \mathcal{W}^{\prime}\right) / p^{n} \mathcal{O}_{\mathfrak{X}_{M}}\left(\mathcal{U}^{\prime}, \mathcal{W}^{\prime}\right)
$$

is injective.

Proof. The lemma is a direct consequence of [A] Miscellany (1.8), (iv) or in this particular case one may reason as follows. We write $\mathcal{O}_{\mathfrak{x}_{M}}(\mathcal{U}, \mathcal{W})=\cup_{i} S_{i}\left(\operatorname{resp} . \mathcal{O}_{\mathfrak{X}_{M}}\left(\mathcal{U}^{\prime}, \mathcal{W}^{\prime}\right)=\cup_{j} S_{j}^{\prime}\right)$ as the union of normal and finite $R_{\mathcal{U}}$-algebras (resp. $R_{\mathcal{U}^{\prime}}$-algebras), étale after inverting $p$ such that for every $i$ there exists $j_{i}$ so that $S_{i}$ is contained in $S_{j_{i}}^{\prime}$ and the map $\operatorname{Spec}\left(S_{j_{i}}^{\prime}\right) \rightarrow \operatorname{Spec}\left(S_{i}\right)$ is surjective on prime ideals containing $p$. Let $x \in S_{i} \cap p^{n} S_{j_{i}}^{\prime}$. Let $\mathcal{P} \subset S_{i}$ be a prime ideal over $p$ and let $\mathcal{P}^{\prime} \subset S_{j_{i}}^{\prime}$ be a height one prime ideal over it. Then $x \in S_{i, \mathcal{P}} \cap p^{n} S_{j_{i}, \mathcal{P}^{\prime}}^{\prime}$. Hence $x \in p^{n} S_{i, \mathcal{P}}$. Thus $x$ lies in the intersection of all height one prime ideals of $S_{i}$ so that $x \in S_{i}$. We conclude that the map $S_{i} / p^{n} S_{i} \rightarrow S_{j_{i}}^{\prime} / p^{n} S_{j_{i}}^{\prime}$ is injective. The claim follows.

The lemma implies that we have an injective map

$$
\bar{R}_{\mathcal{U}} / p^{n} \bar{R}_{\mathcal{U}}=\mathcal{O}_{\mathfrak{X}_{M}}\left(\bar{R}_{\mathcal{U}}\right) / p^{n} \mathcal{O}_{\mathfrak{X}_{M}}\left(\bar{R}_{\mathcal{U}}\right) \longrightarrow\left(\mathcal{O}_{\mathfrak{X}_{M}} / p^{n} \mathcal{O}_{\mathfrak{X}_{M}}\right)\left(\bar{R}_{\mathcal{U}}\right)
$$

The proposition follows then from the following

Lemma 2.17. 1) The cokernel of $\bar{R}_{\mathcal{U}} / p^{n} \bar{R}_{\mathcal{U}} \longrightarrow\left(\mathcal{O}_{\mathfrak{x}_{M}} / p^{n} \mathcal{O}_{\mathfrak{x}_{M}}\right)\left(\bar{R}_{\mathcal{U}}\right)$ is annihilated by the maximal ideal of $\mathcal{O}_{\bar{K}}$.

2) The image of the map $\left(\mathcal{O}_{\mathfrak{x}_{M}} / p^{n+1} \mathcal{O}_{\mathfrak{X}_{M}}\right)\left(\bar{R}_{\mathcal{U}}\right) \rightarrow\left(\mathcal{O}_{\mathfrak{X}_{M}} / p^{n} \mathcal{O}_{\mathfrak{X}_{M}}\right)\left(\bar{R}_{\mathcal{U}}\right)$ factors via

$$
\bar{R}_{\mathcal{U}} / p^{n} \bar{R}_{\mathcal{U}} \subset\left(\mathcal{O}_{\mathfrak{X}_{M}} / p^{n} \mathcal{O}_{\mathfrak{X}_{M}}\right)\left(\bar{R}_{\mathcal{U}}\right) .
$$

3) The image of Frobenius on $\left(\mathcal{O}_{\mathfrak{x}_{M}} / p \mathcal{O}_{\mathfrak{X}_{M}}\right)\left(\bar{R}_{\mathcal{U}}\right)$ factors via $\bar{R}_{\mathcal{U}} / p \bar{R}_{\mathcal{U}} \subset\left(\mathcal{O}_{\mathfrak{X}_{M}} / p \mathcal{O}_{\mathfrak{X}_{M}}\right)\left(\bar{R}_{\mathcal{U}}\right)$.

Proof. It follows from 2.16 that the value of the sheaf $\mathcal{O}_{\mathfrak{X}_{M}} / p^{n} \mathcal{O}_{\mathfrak{X}_{M}}$ on $(\mathcal{U}, \mathcal{W})$ is given by the direct limit, over all coverings $\left(\mathcal{U}^{\prime}, \mathcal{W}^{\prime}\right)$ of $(\mathcal{U}, \mathcal{W})$ with $\mathcal{U}^{\prime}$ affine, of the elements $b$ in $\mathcal{O}_{\mathfrak{X}_{M}}\left(\mathcal{U}^{\prime}, \mathcal{W}^{\prime}\right) / p^{n} \mathcal{O}_{\mathfrak{X}_{M}}\left(\mathcal{U}^{\prime}, \mathcal{W}^{\prime}\right)$ such that the image of $b$ in $\mathcal{O}_{\mathfrak{x}_{M}}\left(\mathcal{U}^{\prime \prime}, \mathcal{W}^{\prime \prime}\right) / p^{n} \mathcal{O}_{\mathfrak{X}_{M}}\left(\mathcal{U}^{\prime \prime}, \mathcal{W}^{\prime \prime}\right)$ is 0 , where $\left(\mathcal{U}^{\prime \prime}, \mathcal{W}^{\prime \prime}\right)$ is the fiber product of $\left(\mathcal{U}^{\prime}, \mathcal{W}^{\prime}\right)$ with itself over $(\mathcal{U}, \mathcal{W})$. Hence

$$
\left(\mathcal{O}_{\mathfrak{X}_{M}} / p^{n} \mathcal{O}_{\mathfrak{X}_{M}}\right)\left(\bar{R}_{\mathcal{U}}\right)=\lim _{S, T} \operatorname{Ker}_{S, T, n}
$$

where the notation is as follows. The direct limit is taken over all normal $R_{\mathcal{U}, \infty}$ sub-algebras $S$ of $\bar{R}_{\mathcal{U}}$, finite and étale after inverting $p$ over $R_{\mathcal{U}, \infty}[1 / p]$, all affine covers $\mathcal{U}^{\prime} \rightarrow \mathcal{U}$ and all normal extensions $R_{\mathcal{U}^{\prime}, \infty} \otimes_{R_{\mathcal{U}}} S \rightarrow T$, finite, étale and Galois after inverting $p$. Eventually, we put $\mathcal{U}^{\prime \prime}:=\operatorname{Spf}\left(R_{\mathcal{U}^{\prime \prime}}\right)$ to be the fiber product of $\mathcal{U}^{\prime}$ with itself over $\mathcal{U}$ i. e., $R_{\mathcal{U}^{\prime \prime}}:=R_{\mathcal{U}^{\prime}} \widehat{\otimes_{R_{\mathcal{U}}}} R_{\mathcal{U}^{\prime}}$. We let $\operatorname{Ker}_{S, T, n}:=\operatorname{Ker}\left(T / p^{n} T \rightrightarrows \widetilde{T \otimes_{S} T} / p^{n} \widetilde{T \otimes_{S} T}\right)$, where $\widetilde{T \otimes_{S} T}$ is the normalization of 


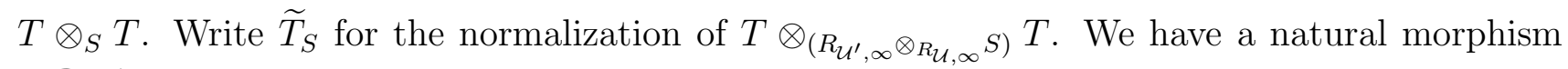
$\widetilde{T \otimes_{S} T} \longrightarrow \widetilde{T}_{S}$ of $R_{\mathcal{U}^{\prime}, \infty}$-algebras. Let

$$
\operatorname{Ker}_{S, T, n}^{\prime}:=\operatorname{Ker}\left(T / p^{n} T \rightrightarrows \widetilde{T}_{S} / p^{n} \widetilde{T}_{S}\right)
$$

Then

$$
\left(\mathcal{O}_{\mathfrak{X}_{M}} / p^{n} \mathcal{O}_{\mathfrak{X}_{M}}\right)\left(\bar{R}_{\mathcal{U}}\right) \subset \lim _{S, T} \operatorname{Ker}_{S, T, n}^{\prime} .
$$

Study of $\operatorname{Ker}_{S, T, n}^{\prime}$. For every $S$ and $T$ as above, write $G_{S, T}$ for the Galois group of $T \otimes_{\mathcal{O}_{K}} K$ over $S \otimes_{R_{\mathcal{U}, \infty}} R_{\mathcal{U}^{\prime}, \infty} \otimes_{\mathcal{O}_{K}} K$. Then $\widetilde{T}_{S}$ is simply the product $\prod_{g \in G_{S, T}} T$. Hence we have

$$
\operatorname{Ker}_{S, T, n}^{\prime}=\operatorname{Ker}\left(T / p^{n} T \rightrightarrows \prod_{g \in G_{S, T}} \frac{T}{p^{n} T}\right)=\left(T / p^{n} T\right)^{G_{S, T}}
$$

where the two maps in the display are $a \mapsto(a, \cdots, a)$ and $a \mapsto(g(a))_{g \in G_{S, T}}$.

Study of $\operatorname{Coker}\left(S / p^{n} S \longrightarrow \operatorname{Ker}_{S, T, n}\right)$. For the rest of this proof we make the following notations: if $B$ is a normal $R_{\mathcal{U}, \infty}$-algebra we denote by $B^{\prime}:=B \otimes_{R_{\mathcal{U}, \infty}} R_{\mathcal{U}^{\prime}, \infty}=B \otimes_{R_{\mathcal{U}}} R_{\mathcal{U}^{\prime}}$, also $B^{\prime \prime}:=B^{\prime} \otimes_{R_{\mathcal{U}^{\prime}, \infty}} R_{\mathcal{U}^{\prime \prime}, \infty}=B^{\prime} \otimes_{R_{\mathcal{U}^{\prime}}} R_{\mathcal{U}^{\prime \prime}}$ (the second equalities above follow from [AI, Lemma 6.19]). Note that $B^{\prime}$ and $B^{\prime \prime}$ are normal. Indeed, $B=\cup B_{i}$ is the union of finite and normal $R_{\mathcal{U}^{\prime}}$-algebras $B_{i}$. Since $R_{\mathcal{U}^{\prime}}$ is an excellent ring by [Va, it follows from [EGAIV, §7.8.3(ii)] that each $B_{i}$ is excellent. Thanks to [EGAIV, $\left.\S 7.8 .3(\mathrm{v})\right]$ we conclude that $B_{i} \otimes_{R_{\mathcal{U}}} R_{\mathcal{U}^{\prime}}$ is normal since it is the $p$-adic completion of an étale $B_{i}$-algebra of finite type. Thus, $B^{\prime}:=B \otimes_{R_{\mathcal{U}}} R_{\mathcal{U}^{\prime}}$ is normal as well. Similarly one shows that $B^{\prime \prime}$ is normal.

We then get a commutative diagram

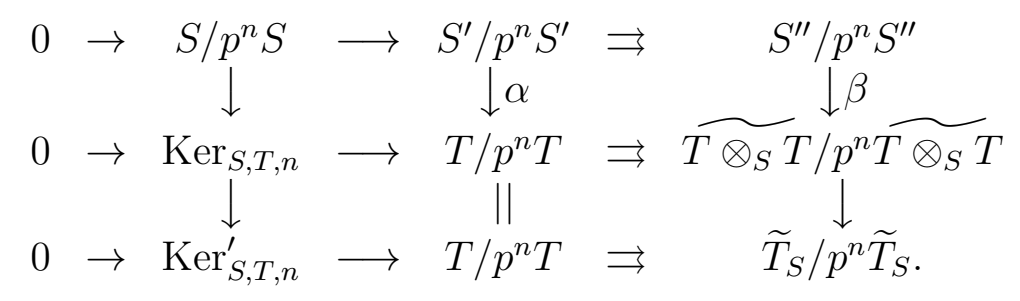

The top row is exact by étale descent and the middle and bottom rows are exact by construction. Since $S^{\prime} \subset T$ and $S^{\prime \prime} \subset T^{\prime \prime}$ are finite extensions of normal rings, the maps $\alpha$ and $\beta$ are injective. Let us remark that the image of $S^{\prime} / p^{n} S^{\prime}$ in $\widetilde{T}_{S} / p^{n} \widetilde{T}_{S}=\widetilde{T \otimes_{S^{\prime}} T / p^{n} T \widetilde{\otimes_{S^{\prime}} T}}$ is 0 , therefore the image of $\alpha$ factors via $\operatorname{Ker}_{S, T, n}^{\prime}=\left(T / p^{n} T\right)^{G_{S, T}}$.

Define $Z$ as $\operatorname{Coker}\left(S^{\prime} / p^{n} S^{\prime} \rightarrow\left(T / p^{n} T\right)^{G_{S, T}}\right) \subset \operatorname{Coker}(\alpha)$ and $Y$ as $\operatorname{Coker}\left(S / p^{n} S \longrightarrow \operatorname{Ker}_{S, T, n}\right)$. Since $\operatorname{Ker}_{S, T, n}$ is $G_{S, T}$-invariant, the image of $Y$ in $\operatorname{Coker}(\alpha)$ is contained in $Z$. Since $\alpha$ and $\beta$ are injective, the map $Y \rightarrow Z$ is injective. Consider the exact sequence

$$
0 \longrightarrow S^{\prime} / p^{n} S^{\prime}=T^{G_{S, T}} / p^{n} T^{G_{S, T}} \longrightarrow\left(T / p^{n} T\right)^{G_{S, T}} \longrightarrow \mathrm{H}^{1}\left(G_{S, T}, T\right) .
$$

Then $Y \subset Z \subset \mathrm{H}^{1}\left(G_{S, T}, T\right)$. Since $R_{\mathcal{U}^{\prime}, \infty} \rightarrow T$ is almost étale, the group $\mathrm{H}^{1}\left(G_{S, T}, T\right)$ is annihilated by any element of the maximal ideal of $\mathcal{O}_{\bar{K}}$; see [F1, Thm. I.2.4(ii)]. This implies the first claim of lemma 2.17. 
Study of the projection $\operatorname{Ker}_{S, T, n+1}^{\prime} \rightarrow \operatorname{Ker}_{S, T, n}^{\prime}$. It is induced by the natural projection $T / p^{n+1} T \rightarrow T / p^{n} T$. Consider the commutative diagram

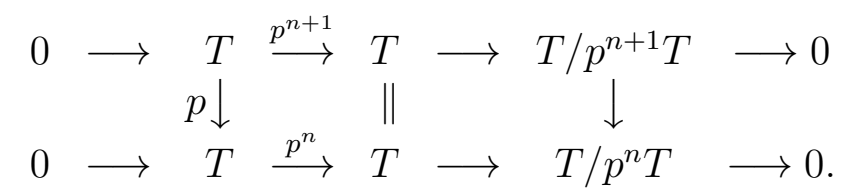

Taking $G_{S, T}$-invariants we get the following commutative diagram:

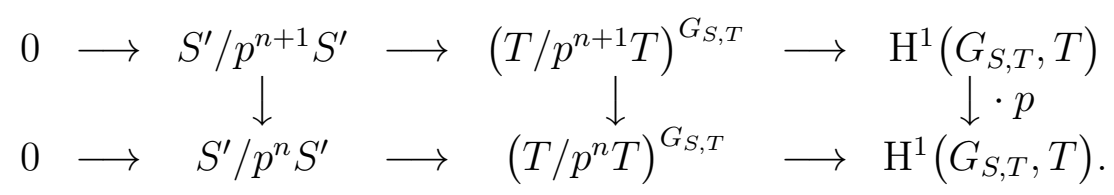

Since multiplication by $p$ annihilates $\mathrm{H}^{1}\left(G_{S, T}, T\right)$, we conclude that the projection $\operatorname{Ker}_{S, T, n+1}^{\prime} \rightarrow$ $\operatorname{Ker}_{S, T, n}^{\prime}$ factors via $S / p^{n} S$. Hence the image of $\left(\mathcal{O}_{\mathfrak{X}_{M}} / p^{n+1} \mathcal{O}_{\mathfrak{X}_{M}}\right)\left(\bar{R}_{\mathcal{U}}\right) \rightarrow\left(\mathcal{O}_{\mathfrak{X}_{M}} / p^{n} \mathcal{O}_{\mathfrak{X}_{M}}\right)\left(\bar{R}_{\mathcal{U}}\right)$ factors via $\bar{R}_{\mathcal{U}} / p^{n} \bar{R}_{\mathcal{U}} \subset\left(\mathcal{O}_{\mathfrak{X}_{M}} / p^{n} \mathcal{O}_{\mathfrak{X}_{M}}\right)\left(\bar{R}_{\mathcal{U}}\right)$. This proves the second claim of the lemma.

Consider the map of sets $T / p T \rightarrow T / p^{2} T$ sending an element $a$ to the $p$-th power $\widetilde{a}^{p}$ of a lift $\widetilde{a}$ of $a$ in $T / p^{2} T$. It is well defined since it does not depend on the choice of the lift $\widetilde{a}$. It induces a map $\rho:(T / p T)^{G_{S, T}} \rightarrow\left(T / p^{2} T\right)^{G_{S, T}}$. Frobenius on $(T / p T)^{G_{S, T}}$ factors as the composite of $\rho$ and the projection $\left(T / p^{2} T\right)^{G_{S, T}} \rightarrow(T / p T)^{G_{S, T}}$. It follows from the above discussion that Frobenius $\operatorname{Ker}_{S, T, 1}^{\prime} \rightarrow \operatorname{Ker}_{S, T, 1}^{\prime}$ factors via $S / p S$. Hence the image of Frobenius on $\left(\mathcal{O}_{\mathfrak{X}_{M}} / p \mathcal{O}_{\mathfrak{X}_{M}}\right)\left(\bar{R}_{\mathcal{U}}\right)$ factors via $\bar{R}_{\mathcal{U}} / p \bar{R}_{\mathcal{U}} \subset\left(\mathcal{O}_{\mathfrak{X}_{M}} / p \mathcal{O}_{\mathfrak{X}_{M}}\right)\left(\bar{R}_{\mathcal{U}}\right)$. This proves the last claim of the lemma and the proposition 2.15 .

The map $\theta_{M}$. We define a morphism $\theta_{M}: \mathbb{A}_{\text {inf,M }}^{+} \longrightarrow \widehat{\mathcal{O}}_{\mathfrak{X}_{M}}$ of objects of $\operatorname{Sh}\left(\mathfrak{X}_{M}\right)^{\mathbb{N}}$ as follows.

We work in the formal setting. Fix a non-negative integer $n$. Let $(\mathcal{U}, \mathcal{W})$ be an object of $\mathfrak{X}_{M}$. Let $S=\mathcal{O}_{\mathfrak{x}_{M}}(\mathcal{U}, \mathcal{W})$ and consider the diagram of sets and maps

$$
\begin{aligned}
& \left(S / p^{n} S\right)^{n} \stackrel{a_{n}}{\longrightarrow} S / p^{n} S \\
& \downarrow b_{n} \\
& (S / p S)^{n}
\end{aligned}
$$

where $a_{n}\left(s_{0}, s_{1}, \ldots, s_{n-1}\right):=\sum_{i=0}^{n-1} p^{i} s_{i}^{p^{n-1-i}}$ and $b_{n}$ is the natural projection. Remark that there is a unique map of sets, $c_{n}:(S / p S)^{n} \longrightarrow S / p^{n} S$ which makes the diagram commutative, i.e. such that $c_{n} \circ b_{n}=a_{n}$. Moreover, $c_{n}$ induces a ring homomorphism $c_{n,(\mathcal{U}, \mathcal{W})}: \mathbb{W}_{n}(S / p S) \longrightarrow S / p^{n} S$ functorial in $(\mathcal{U}, \mathcal{W})$ i.e. a morphism of presheaves $\mathbb{W}_{n, M} \stackrel{c_{n}}{\longrightarrow} \mathcal{O}_{\mathfrak{X}_{M}} / p^{n} \mathcal{O}_{\mathfrak{X}_{M}}$. Denote by $\theta_{M, n}$ the induced morphism on the associated sheaves.

Lemma 2.18. a) The following diagram of sheaves and morphisms commutes for varying $n \in \mathbb{N}$ :

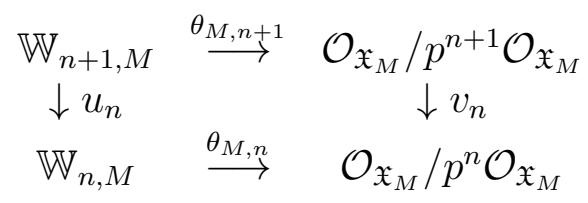


where $u_{n}$ is the composition of the natural projection and Frobenius and $v_{n}$ is the natural projection.

b) For $\mathcal{U}=\operatorname{Spf}\left(R_{\mathcal{U}}\right)$ connected open affine in $X^{\mathrm{et}}$, the localization of

$$
\theta_{M}=\left\{\theta_{M, n}\right\}: \mathbb{A}_{\text {inf,M }}^{+} \longrightarrow \widehat{\overline{\mathcal{O}}}_{\mathfrak{X}_{M}}
$$

is the map $\theta_{\mathcal{U}}: A_{\mathrm{inf}, \mathrm{M}}^{+}\left(\bar{R}_{\mathcal{U}}\right) \longrightarrow \widehat{\bar{R}}_{\mathcal{U}}$ of [Bril, Prop. 5.1.1].

Proof. a) It is enough to prove that the diagram commutes at the level of pre-sheaves, so let as before $(\mathcal{U}, \mathcal{W})$ be an object of $\mathfrak{X}_{M}$ and let $S=\mathcal{O}_{\mathfrak{X}_{M}}(\mathcal{U}, \mathcal{W})$. We are reduced to checking that the following diagram of sets and maps commutes

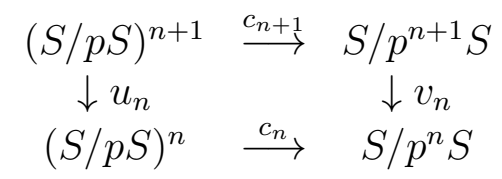

where $u_{n}\left(s_{0}, s_{1}, \ldots, s_{n}\right)=\left(s_{0}^{p}, s_{1}^{p}, \ldots, s_{n-1}^{p}\right)$. This is a simple calculation which we leave to the reader.

b) Using the proposition 2.15, the map $\left(\theta_{n, M}\right)_{\mathcal{U}}$ is induced by $\lim _{\rightarrow, \mathcal{W}} c_{n,(\mathcal{U}, \mathcal{W})}$ and therefore coincides with the map defined in [Bri, Prop. 5.1.1].

Fix an object $(\mathcal{U}, \mathcal{W})$ of $\mathfrak{X}_{M}$. Write $S=\mathcal{O}_{\mathfrak{X}_{M}}(\mathcal{U}, \mathcal{W})$.

Lemma 2.19. Assume that $p^{1 / p^{n-1}} \in S$. Then $\xi_{n}:=\left[p^{1 / p^{n-1}}\right]-p$ (see section 1.2 ) is a well defined element of $\mathbb{W}_{n}(S / p S)$ and it generates the kernel of $c_{n}: \mathbb{W}_{n}(S / p S) \longrightarrow S / p^{n} S$.

Proof. Let us first remark that $c_{n}\left(\xi_{n}\right)=\left(p^{1 / p^{n-1}}\right)^{p^{n-1}}-p=0$, therefore $\xi_{n} \in \operatorname{Ker}\left(c_{n}\right)$.

We show that if $x \in \operatorname{Ker}\left(c_{n}\right)$ then $x \in \xi_{n} \mathbb{W}_{n}(S / p S)$. We'll prove this statement by induction on $n$. For $n=1, c_{1}=\mathrm{Id}$ and $\xi_{1}=0 \in \mathbb{W}_{1}(S / p S)=S / p S$. Let now $n>1$ and suppose that our statement is true for $n-1$. Let $\alpha \in \operatorname{Ker}\left(c_{n}\right)$.

Claim 1 There are $\beta \in \mathbb{W}_{n}(S / p S)$ and $\gamma \in \mathbb{W}_{n-1}(S / p S)$ such that $\alpha=\xi_{n} \beta+\mathbb{V}(\gamma)$, where $\mathbb{V}: \mathbb{W}_{n-1}(S / p S) \longrightarrow \mathbb{W}_{n}(S / p S)$ is Vershiebung, i.e. $\mathbb{V}\left(s_{0}, s_{1}, \ldots, s_{n-2}\right)=\left(0, s_{0}, s_{1}, \ldots, s_{n-2}\right)$, for $\left(s_{0}, s_{1}, \ldots, s_{n-2}\right) \in \mathbb{W}_{n-1}(S / p S)$.

To prove this claim let us write $\alpha=\left(\alpha_{0}, \alpha_{1}, \ldots, \alpha_{n-1}\right)$ and so $0=c_{n}(\alpha)=\sum_{i=0}^{n-1} p^{i} \widetilde{\alpha}_{i}^{p^{n-1-i}}$, where if $x \in S / p S$, then $\widetilde{x}$ denotes any lift of $x$ to $S / p^{n} S$. Therefore $\widetilde{\alpha}_{0}^{p^{n-1}}=p c$ for some $c \in S$. Let $R_{\mathcal{U}} \subset S^{\prime}(\subset S)$ be a finite and normal extension containing both $\widetilde{\alpha}_{0}$ and $p^{1 / p^{n-1}}$. For every height one prime ideal $\mathfrak{p}$ of $S^{\prime}$ we have $\widetilde{\alpha}_{0}^{p^{n-1}} / p=c$ which lies in $S_{\mathfrak{p}}^{\prime}$ so that $\widetilde{\alpha}_{0} / p^{1 / p^{n-1}} \in S_{\mathfrak{p}}^{\prime}$ since the latter is a dvr. We conclude that $\widetilde{\alpha}_{0} / p^{1 / p^{n-1}}$ lies in the intersection of the localizations of $S^{\prime}$ at every height one prime ideal so that, since $S^{\prime}$ is noetherian, we must have $\widetilde{\alpha}_{0} / p^{1 / p^{n-1}} \in S^{\prime}$. Denote by $\beta_{0}$ the image of $\widetilde{\alpha}_{0} / p^{1 / p^{n-1}}$ in $S / p S$. Then $\alpha_{0}=p^{1 / p^{n-1}} \beta_{0}$ in $S / p S$. Let $\beta:=\left(\beta_{0}, 0, \ldots, 0\right) \in$ $\mathbb{W}_{n}(S / p S)$ and let us compute: $\alpha-\xi_{n} \cdot \beta=\alpha-\widetilde{p}_{n} \cdot \beta+p \beta=\left(\alpha_{0}, \alpha_{1}, \ldots, \alpha_{n-1}\right)-\left(\alpha_{0}, 0, \ldots, 0\right)+$ $\left(0, \beta_{0}^{p}, 0, \ldots, 0\right) \in \mathbb{V}\left(\mathbb{W}_{n-1}(S / p S)\right)$.

Let $\gamma \in \mathbb{W}_{n-1}(S / p S)$ be such that $\alpha-\xi_{n} \beta=\mathbb{V}(\gamma)$. Then $c_{n}(\mathbb{V}(\gamma))=c_{n}\left(\alpha-\xi_{n} \beta\right)=0$ and $c_{n}(\mathbb{V}(\gamma))=w_{n}\left(c_{n-1}(\gamma)\right)$, where $w_{n}$ is the isomorphism $w_{n}: S / p^{n-1} S \cong p S / p^{n} S$. Therefore 
$c_{n-1}(\gamma)=0$ and by the inductive hypothesis there is $\delta \in \mathbb{W}_{n-1}(S / p S)$ such that $\gamma=\xi_{n-1} \delta$. The lemma now follows from

Claim $2 \mathbb{V}\left(\xi_{n-1} \delta\right)=\xi_{n} \mathbb{V}(\delta)$. This is a simple calculation with Witt vectors. Write $\delta=$ $\left(\delta_{0}, \delta_{1}, \ldots, \delta_{n-2}\right)$, then

$$
\begin{aligned}
\xi_{n-1} \delta & =\left(p^{1 / p^{n-2}}, 0, \ldots, 0\right) \cdot\left(\delta_{0}, \delta_{1}, \ldots, \delta_{n-2}\right)-p \delta= \\
& =\left(p^{1 / p^{n-2}} \delta_{0}, p^{1 / p^{n-3}} \delta_{1}, \ldots, p^{1 / p} \delta_{n-2}\right)-p \delta
\end{aligned}
$$

Therefore

$$
\mathbb{V}\left(\xi_{n-1} \delta\right)=\left(0, p^{1 / p^{n-2}} \delta_{0}, \ldots, p^{1 / p} \delta_{n-2}\right)-\mathbb{V}(p \delta) .
$$

On the other hand,

$$
\xi_{n} \mathbb{V}(\delta)=\xi_{n} \cdot\left(0, \delta_{0}, \delta_{1}, \ldots, \delta_{n-2}\right)=\left(0, p^{1 / p^{n-2}} \delta_{0}, \ldots, p^{1 / p} \delta_{n-2}\right)-p(\mathbb{V} \delta),
$$

which proves the second claim and the lemma because $\mathbb{V}$ is an additive map.

Note that if $(\mathcal{U}, \mathcal{W})$ is an object of $\mathfrak{X}_{\bar{K}}$ then $\mathcal{O}_{\bar{K}} \subset S$ and $\widetilde{p}_{n} \in \mathbb{W}_{n}(S / p S)$. In particular $\xi$ is naturally a section of the sheaf $\mathbb{A}_{\text {inf }}^{+}\left(\mathcal{O}_{\mathfrak{X}}\right)$ over $(\mathcal{U}, \mathcal{W})$. We deduce from 2.19.

Corollary 2.20. We have $\operatorname{Ker}\left(\theta_{\bar{K}}: \mathbb{A}_{\text {inf }} \longrightarrow \widehat{\overline{\mathcal{O}}}_{\mathfrak{X}_{\bar{K}}}\right)=\xi \cdot \mathbb{A}_{\text {inf }}$ as sheaves in $\operatorname{Sh}\left(\mathfrak{X}_{\bar{K}}\right)^{\mathbb{N}}$.

Let $\mathcal{U}$ be a small affine as in $\oint 2.2$. Write $q^{\prime}:=\frac{[\varepsilon]^{\frac{1}{1}}}{[\varepsilon]^{\frac{1}{p}}-1}=1+[\varepsilon]^{\frac{1}{p}}+\cdots+[\varepsilon]^{\frac{p-1}{p}} \in A_{\mathrm{inf}}^{+}$. Then

Lemma 2.21. (1) For every positive integer $r$ the Frobenius morphism $\varphi$ induces an isomorphism $\mathbb{W}_{n}\left(\bar{R}_{\mathcal{U}} / p \bar{R}_{\mathcal{U}}\right) / \varphi^{-r-1}\left(q^{\prime}\right) \mathbb{W}_{n}\left(\bar{R}_{\mathcal{U}} / p \bar{R}_{\mathcal{U}}\right) \cong \mathbb{W}_{n}\left(\bar{R}_{\mathcal{U}} / p \bar{R}_{\mathcal{U}}\right) / \varphi^{-r}\left(q^{\prime}\right) \mathbb{W}_{n}\left(\bar{R}_{\mathcal{U}} / p \bar{R}_{\mathcal{U}}\right)$

(2) for every $n \in \mathbb{N}$ the $W_{n}-$ module $\mathbb{W}_{n}\left(\bar{R}_{\mathcal{U}} / p \bar{R}_{\mathcal{U}}\right)$ is flat;

(3) assume that we are in the formal case. The sequence $0 \longrightarrow \mathbb{Z} / p^{n} \mathbb{Z} \longrightarrow \mathbb{W}_{n}\left(\bar{R}_{\mathcal{U}} / p \bar{R}_{\mathcal{U}}\right) \stackrel{\varphi-1}{\longrightarrow}$ $\mathbb{W}_{n}\left(\bar{R}_{\mathcal{U}} / p \bar{R}_{\mathcal{U}}\right) \longrightarrow 0$ is exact where $\mathbb{Z} / p^{n} \mathbb{Z}$ is the constant group over $\operatorname{Spec}\left(R_{\mathcal{U}} \otimes_{\mathcal{O}_{K}} M\right)$.

Proof. (1) We proceed by induction on $n$. The kernel of the reduction $\mathbb{W}_{n+1}\left(\bar{R}_{\mathcal{U}} / p \bar{R}_{\mathcal{U}}\right) \rightarrow$ $\mathbb{W}_{n}\left(\bar{R}_{\mathcal{U}} / p \bar{R}_{\mathcal{U}}\right)$ is $\mathbb{V}^{n} \mathbb{W}_{n+1}\left(\bar{R}_{\mathcal{U}} / p \bar{R}_{\mathcal{U}}\right)$, where $\mathbb{V}$ is the Vershiebung. The latter is isomorphic to $\bar{R}_{\mathcal{U}} / p \bar{R}_{\mathcal{U}}$ as an abelian group with structure of $\mathbb{W}_{n+1}\left(\bar{R}_{\mathcal{U}} / p \bar{R}_{\mathcal{U}}\right)$-module via the map $\mathbb{W}_{n+1}\left(\bar{R}_{\mathcal{U}} / p \bar{R}_{\mathcal{U}}\right) \rightarrow$ $\bar{R}_{\mathcal{U}} / p \bar{R}_{\mathcal{U}},\left(a_{0}, \ldots, a_{n}\right) \mapsto a_{0}^{p^{n}}$

Let $A_{r, n}$ be the assertion that $\varphi$ induces an isomorphism

$$
\mathbb{W}_{n}\left(\bar{R}_{\mathcal{U}} / \bar{R}_{\mathcal{U}}\right) / \varphi^{-r-1}\left(q^{\prime}\right) \mathbb{W}_{n}\left(\bar{R}_{\mathcal{U}} / \bar{R}_{\mathcal{U}}\right) \longrightarrow \mathbb{W}_{n}\left(\bar{R}_{\mathcal{U}} / \bar{R}_{\mathcal{U}}\right) / \varphi^{-r}\left(q^{\prime}\right) \mathbb{W}_{n}\left(\bar{R}_{\mathcal{U}} / \bar{R}_{\mathcal{U}}\right)
$$

Let $B_{r, n}$ be the following assertion: the sheaf

$$
\mathbb{V}^{n} \mathbb{W}_{n+1}\left(\bar{R}_{\mathcal{U}} / p \bar{R}_{\mathcal{U}}\right) / \varphi^{-r}\left(q^{\prime}\right) \mathbb{V}^{n} \mathbb{W}_{n+1}\left(\bar{R}_{\mathcal{U}} / p \bar{R}_{\mathcal{U}}\right)
$$

which is isomorphic to $\bar{R}_{\mathcal{U}} /\left(\left(1+\zeta_{2}+\cdots \zeta_{2}^{p-1}\right)^{\frac{1}{p^{r-n}}}, p\right) \bar{R}_{\mathcal{U}}$, injects in

$$
\mathbb{W}_{n+1}\left(\bar{R}_{\mathcal{U}} / p \bar{R}_{\mathcal{U}}\right) / \varphi^{-r}\left(q^{\prime}\right) \mathbb{W}_{n+1}\left(\bar{R}_{\mathcal{U}} / p \bar{R}_{\mathcal{U}}\right)
$$


We prove by induction on $r$ and $n$ that the claims $A_{r, n}$ and $B_{r, n}$ hold. For $n=1$ and any $r$ the fact that the map $\varphi$ is injective follows from the fact that $\bar{R}_{\mathcal{U}}$ is normal, cf. proof of 2.16, It is surjective by [Bri, Prop. 2.0.1]. Thus $A_{r, 1}$ holds. Since $1+\zeta_{2}+\cdots \zeta_{2}^{p-1}$ has $p$-adic valuation 1 assertion $B_{r, n}$ holds for every $n \in \mathbb{N}$ and every $r \geq n$. It then follows by descending induction on $r<n$ and from $A_{r, 1}$ that $B_{r+1, n}$ implies $B_{r, n}$. Thus $B_{r, n}$ holds for every $r$ and $n$. Proceeding by induction on $n$ one then proves that $A_{r, n}$ and $B_{r, n+1}$ together with $A_{r, 1}$ imply $A_{r, n+1}$.

(2) It suffices to prove that the map $J \otimes_{W_{n}} \mathbb{W}_{n}\left(\bar{R}_{\mathcal{U}} / p \bar{R}_{\mathcal{U}}\right) \rightarrow \mathbb{W}_{n}\left(\bar{R}_{\mathcal{U}} / p \bar{R}_{\mathcal{U}}\right)$ is injective for every finitely generated ideal $J \subset W_{n}$. We proceed by induction on $n \in \mathbb{N}$. Let $n=1$. Since $\mathcal{O}_{\bar{K}}$ is the union of discrete valuation rings and $J$ is finitely generated we have $J=p^{\delta} \mathcal{O}_{\bar{K}} / p \mathcal{O}_{\bar{K}}$ for some $0 \leq \delta \leq 1$ i. e., $J \cong \mathcal{O}_{\bar{K}} / p^{1-\delta} \mathcal{O}_{\bar{K}}$ and the inclusions $J \subset \mathcal{O}_{\bar{K}} / p \mathcal{O}_{\bar{K}}$ is given by multiplication by $\cdot p^{\delta}: \mathcal{O}_{\bar{K}} / p^{1-\delta} \mathcal{O}_{\bar{K}} \rightarrow \mathcal{O}_{\bar{K}} / p \mathcal{O}_{\bar{K}}$. Then $J \otimes_{\mathcal{O}_{\bar{K}}} \bar{R}_{\mathcal{U}} / p \bar{R}_{\mathcal{U}}=\bar{R}_{\mathcal{U}} / p^{1-\delta} \bar{R}_{\mathcal{U}}$ and the map $J \otimes_{\mathcal{O}_{K}} \bar{R}_{\mathcal{U}} / p \bar{R}_{\mathcal{U}} \rightarrow \bar{R}_{\mathcal{U}} / p \bar{R}_{\mathcal{U}}$ is the map $\bar{R}_{\mathcal{U}} / p^{1-\delta} \bar{R}_{\mathcal{U}} \rightarrow \bar{R}_{\mathcal{U}} / p \bar{R}_{\mathcal{U}}$ given by $s \mapsto p^{\delta} s$. Since $\bar{R}_{\mathcal{U}}$ is normal, this map is injective as well.

Assume that the claim holds for ideals of $W_{n}$ for $n \leq N$. Let $J \subset W_{N+1}$ be an ideal. Let $\pi_{N}: W_{N+1} \rightarrow W_{N}$ be the natural projection. Its kernel is $\mathbb{V}^{N} W_{N+1}$ which is isomorphic to $\mathcal{O}_{\bar{K}} / p \mathcal{O}_{\bar{K}}$. Let $J_{N}$ be the image of $J$ via $\pi_{N}$ and put $J^{\prime}:=J \cap \operatorname{Ker}\left(\pi_{N}\right)$ which we view as an ideal of $\mathcal{O}_{\bar{K}} / p \mathcal{O}_{\bar{K}}$ via the identification above. Since Frobenius is surjective on $\bar{R}_{\mathcal{U}} / p \bar{R}_{\mathcal{U}}$, and hence on $\mathbb{W}_{N+1}\left(\bar{R}_{\mathcal{U}} / p \bar{R}_{\mathcal{U}}\right)$, and multiplication by $p$ is $\mathbb{V} \circ \varphi$, we have $\mathbb{V} \mathbb{W}_{N+1}\left(\bar{R}_{\mathcal{U}} / p \bar{R}_{\mathcal{U}}\right)=$ $p \mathbb{W}_{N+1}\left(\bar{R}_{\mathcal{U}} / p \bar{R}_{\mathcal{U}}\right)$. Then $J^{\prime} \otimes_{W_{N+1}} \mathbb{W}_{N+1}\left(\bar{R}_{\mathcal{U}} / p \bar{R}_{\mathcal{U}}\right) \cong J^{\prime} \otimes_{\mathcal{O}_{\bar{K}} / p \mathcal{O}_{\bar{K}}} \bar{R}_{\mathcal{U}} / p \bar{R}_{\mathcal{U}}$ which is isomorphic to $J^{\prime} \otimes_{\mathcal{O}_{K} / p \mathcal{O}_{\bar{K}}} \bar{R}_{\mathcal{U}} /\left(\varphi^{-N+1}\left(q^{\prime}\right), p\right) \bar{R}_{\mathcal{U}}$ since multiplication by $\varphi^{-N+1}\left(q^{\prime}\right)$ is multiplication by $p \equiv 0$ on $J^{\prime}$. Moreover the map from $J^{\prime} \otimes_{\mathcal{O}_{\bar{K}} / p \mathcal{O}_{\bar{K}}} \bar{R}_{\mathcal{U}} /\left(\varphi^{-N+1}\left(q^{\prime}\right), p\right) \bar{R}_{\mathcal{U}}$ to $\mathbb{W}_{N+1}\left(\bar{R}_{\mathcal{U}} / p \bar{R}_{\mathcal{U}}\right)$ factors via $\bar{R}_{\mathcal{U}} / p \bar{R}_{\mathcal{U}} \cong \mathbb{V}^{N} \mathbb{W}_{N+1}\left(\bar{R}_{\mathcal{U}} / p \bar{R}_{\mathcal{U}}\right)$ and via these identifications it is the map $a \otimes b \mapsto a b^{p^{N-1}}$. This is proven to be injective as in the proof of the $n=1$ case.

We have an exact sequence $0 \rightarrow J^{\prime} \rightarrow J \rightarrow J_{N} \rightarrow 0$ of $W_{N+1}$-modules. The induced map $J^{\prime} \otimes_{W_{N+1}} \mathbb{W}_{N+1}\left(\bar{R}_{\mathcal{U}} / p \bar{R}_{\mathcal{U}}\right) \rightarrow \mathbb{V}^{N} \mathbb{W}_{N+1}\left(\bar{R}_{\mathcal{U}} / p \bar{R}_{\mathcal{U}}\right)$ has been proven to be injective. Note that $J_{N} \otimes_{W_{N+1}} \mathbb{W}_{N+1}\left(\bar{R}_{\mathcal{U}} / p \bar{R}_{\mathcal{U}}\right) \cong J_{N} \otimes_{W_{N}} \mathbb{W}_{N}\left(\bar{R}_{\mathcal{U}} / p \bar{R}_{\mathcal{U}}\right)$ since the kernel $\mathbb{V}^{N} \mathbb{W}_{N+1}\left(\bar{R}_{\mathcal{U}} / p \bar{R}_{\mathcal{U}}\right)=$ $\mathbb{V}^{N} \varphi^{N} \mathbb{W}_{N+1}\left(\bar{R}_{\mathcal{U}} / p \bar{R}_{\mathcal{U}}\right)=p^{N} \mathbb{W}_{N+1}\left(\bar{R}_{\mathcal{U}} / p \bar{R}_{\mathcal{U}}\right)$ and $p^{N} \equiv 0 \in W_{N}$. Furthermore, the map $J_{N} \otimes_{W_{N}}$ $\mathbb{W}_{N}\left(\bar{R}_{\mathcal{U}} / p \bar{R}_{\mathcal{U}}\right) \rightarrow \mathbb{W}_{N}\left(\bar{R}_{\mathcal{U}} / p \bar{R}_{\mathcal{U}}\right)$ is injective by inductive hypothesis. Consider the following commutative diagram

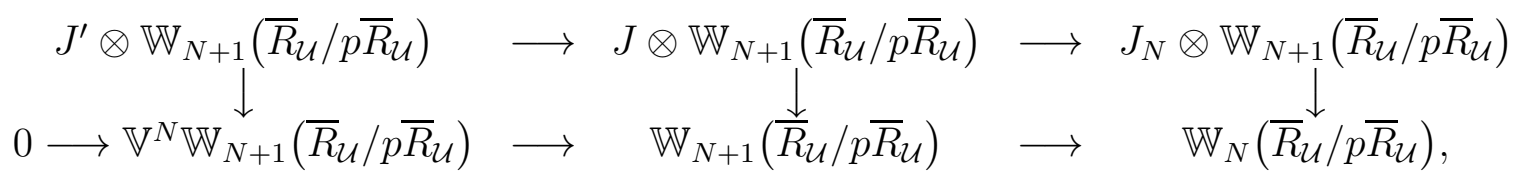

where in the first row the tensor products are over the ring $W_{N+1}$. The rows are exact and we have proven that the left and right vertical maps are injective. Therefore the map $J \otimes_{W_{N+1}}$ $\mathbb{W}_{N+1}\left(\bar{R}_{\mathcal{U}} / p \bar{R}_{\mathcal{U}}\right) \rightarrow \mathbb{W}_{N+1}\left(\bar{R}_{\mathcal{U}} / p \bar{R}_{\mathcal{U}}\right)$ is injective as well.

(3) Proceeding by induction on $n$ it suffices to prove the claim for $n=1$ i. e., that the sequence $0 \longrightarrow \mathbb{F}_{p} \longrightarrow \bar{R}_{\mathcal{U}} / p \bar{R}_{\mathcal{U}} \stackrel{\varphi-1}{\longrightarrow} \bar{R}_{\mathcal{U}} / p \bar{R}_{\mathcal{U}} \longrightarrow 0$ is exact. Since $R_{\mathcal{U}}$ is $p$-adically complete every finite extensions of $R_{\mathcal{U}}$ is also $p$-adically complete and by Hensel's lemma the connected components of the associated spectrum are in bijection with the connected components of its reduction modulo $p$. In particular the scheme $\operatorname{Spec}\left(\bar{R}_{\mathcal{U}} / p \bar{R}_{\mathcal{U}}\right)$ has as many connected components as $\operatorname{Spec}\left(\bar{R}_{\mathcal{U}} \otimes_{\mathcal{O}_{K}} \mathcal{O}_{M}\right)$ has. By construction these coincide with the connected components of $\operatorname{Spec}\left(R_{\mathcal{U}} \otimes_{\mathcal{O}_{K}} M\right)$. The exactness on the left and in the middle follow from Artin-Schreier theory. 
The cokernel of $\varphi-1$ on $\bar{R}_{\mathcal{U}} / p \bar{R}_{\mathcal{U}}$ is contained in $\mathrm{H}^{1}\left(\bar{R}_{\mathcal{U}} / p \bar{R}_{\mathcal{U}}, \mathbb{F}_{p}\right)$ by Artin-Schreier theory. Let $\bar{Z} \rightarrow \operatorname{Spec}\left(\bar{R}_{\mathcal{U}} / p \bar{R}_{\mathcal{U}}\right)$ be an $\mathbb{F}_{p}$-torsor. It can be lifted to an $\mathbb{F}_{p}$-torsor $Z \rightarrow \operatorname{Spec}(S)$ over a finite and normal extension $R_{\mathcal{U}} \subset S$, étale after inverting $p$. In particular $Z=\operatorname{Spec}(T)$ is affine with $T$ normal so that $Z\left(\bar{R}_{\mathcal{U}}\right)$ admits a section by definition of $\bar{R}_{\mathcal{U}}$. Then $\bar{Z}$ admits a section as well and thus it is the trivial torsor. Therefore $\mathrm{H}^{1}\left(\bar{R}_{\mathcal{U}} / p \bar{R}_{\mathcal{U}}, \mathbb{F}_{p}\right)=0$ and the claim follows.

Corollary 2.22. For every $n$ the sheaf $\mathbb{W}_{n, \bar{K}}$ is a sheaf of flat $W_{n}$-modules. Furthermore, $\varphi$ induces an isomorphism $\mathbb{W}_{n, \bar{K}} /\left([\varepsilon]^{\frac{1}{p^{r+1}}}-1\right) \mathbb{W}_{n, \bar{K}} \longrightarrow \mathbb{W}_{n, \bar{K}} /\left([\varepsilon]^{\frac{1}{p^{r}}}-1\right) \mathbb{W}_{n, \bar{K}}$ for every $r \in \mathbb{N}$. In the formal case the sequence $0 \longrightarrow \mathbb{Z}_{p} \longrightarrow \mathbb{W}_{n, \bar{K}} \stackrel{\varphi-1}{\longrightarrow} \mathbb{W}_{n, \bar{K}} \longrightarrow 0$ is exact.

Proof. It suffices to show the claims for the pre-sheaf $\mathbb{W}_{n}\left(\mathcal{O}_{\mathfrak{X}_{\bar{K}}} / p \mathcal{O}_{\mathfrak{X}_{\bar{K}}}\right)$ and further after passing to its localizations at every small affine $\mathcal{U} \subset X$. The claims follow from lemma 2.21,

\subsection{The sheaf $\mathbb{A} \underset{\text { cris, } M}{\nabla}$.}

We start with a formal definition. A $\mathbb{W}(k)$-divided power $(\mathbb{W}(k)-\mathrm{DP})$ sheaf of algebras in $\operatorname{Sh}\left(\mathfrak{X}_{M}\right)$ or $\operatorname{Sh}\left(\mathfrak{X}_{M}\right)^{\mathbb{N}}$ is a triple $(\mathcal{F}, \mathcal{I}, \gamma)$ consisting of $(1)$ a sheaf of $\mathbb{W}(k)$-algebras $\mathcal{F} \in \operatorname{Sh}\left(\mathfrak{X}_{M}\right)$ (resp. an inverse system of sheaves of $\mathbb{W}(k)$-algebras $\left.\left\{\mathcal{F}_{n}\right\} \in \operatorname{Sh}\left(\mathfrak{X}_{M}\right)^{\mathbb{N}}\right)$, (2) a sheaf of ideals $\mathcal{I} \subset \mathcal{F}$ (resp. an inverse system of sheaves of ideals $\left\{\mathcal{I}_{n} \subset \mathcal{F}_{n}\right\}$ ), (3) maps $\gamma_{i}: \mathcal{I} \rightarrow \mathcal{I}$ for $i \in \mathbb{N}$ such that for every object $(\mathcal{U}, \mathcal{W})$ the triple $\left(\mathcal{F}(\mathcal{U}, \mathcal{W}), \mathcal{I}(\mathcal{U}, \mathcal{W}), \gamma_{(\mathcal{U}, \mathcal{W})}\right)$ (resp. for every $n$ the triple $\left.\left(\mathcal{F}_{n}(\mathcal{U}, \mathcal{W}), \mathcal{I}_{n}(\mathcal{U}, \mathcal{W}), \gamma_{(\mathcal{U}, \mathcal{W})}\right)\right)$ is a DP algebra compatible with the standard DP structure on the ideal $p \mathbb{W}(k)$ in the sense of [BO, Ch. 3]. Given a sheaf of $\mathbb{W}(k)$-algebras $\mathcal{G}$ and an ideal $\mathcal{J} \subset \mathcal{G}$ (resp. an inverse system of sheaves of $\mathbb{W}(k)$-algebras $\mathcal{G}$ and ideals $\mathcal{J} \subset \mathcal{G})$ the $\mathbb{W}(k)$-divided power envelope of $\mathcal{G}$ with respect to $\mathcal{J}$ is a $\mathbb{W}(k)$-DP sheaf of algebras $(\mathcal{F}, \mathcal{I}, \gamma)$ and a morphism $\mathcal{G} \rightarrow \mathcal{F}$ of sheaves (or inverse systems of sheaves) of $\mathbb{W}(k)$-algebras, such that $\mathcal{J}$ maps to $\mathcal{I}$, which is universal for morphisms as sheaves (or inverse systems of sheaves) of $\mathbb{W}(k)$-algebras from $\mathcal{G}$ to $\mathbb{W}(k)-\mathrm{DP}$ sheaves of algebras $\mathcal{F}^{\prime}$ such that $\mathcal{J}$ maps to the sheaf of ideals of $\mathcal{F}^{\prime}$ on which the divided power structure is defined.

We'd like to consider the $\mathbb{W}(k)$-DP envelope of the sheaf $\mathbb{A}_{\text {inf }, M}^{+} \in \operatorname{Sh}\left(\mathfrak{X}_{M}\right)$ with respect to the sheaf of ideals $\operatorname{Ker}\left(\theta_{M}\right)$. One could use the general machinery of [B1, Thm. I.2.4.1] to guarantee that it exists but we prefer to provide a different more explicit description. We start with:

Lemma 2.23. Let $\mathcal{G}$ be a sheaf of $\mathbb{W}(k)$-algebras and let $\mathcal{J} \subset \mathcal{G}$ be an ideal. Assume that the $\mathbb{W}(k)$-divided power envelope $(\mathcal{F}, \mathcal{I}, \gamma)$ of $\mathcal{G}$ with respect to $\mathcal{I}$ exists. Then for every $\mathcal{U} \in X^{\text {et }}$ the restriction of $(\mathcal{F}, \mathcal{I}, \gamma)$ to $\mathfrak{U}_{M}$ is the $\mathbb{W}(k)$-divided power $(D P)$ envelope of $\left.\mathcal{G}\right|_{\mathfrak{L}_{M}}$ with respect to $\left.\mathcal{I}\right|_{\mathfrak{U}_{M}}$.

Proof. Let $j: \mathfrak{X}_{M} \rightarrow \mathfrak{U}_{M}$ be the continuous morphism of sites sending $(\mathcal{V}, \mathcal{W}) \mapsto(\mathcal{V}, \mathcal{W}) \times_{\left(\mathfrak{X}, \mathfrak{x}_{K}\right)}$ $\left(\mathcal{U}, \mathcal{U}_{K}\right)$. Let $j_{!}: \operatorname{Sh}\left(\mathfrak{U}_{M}\right) \rightarrow \operatorname{Sh}\left(\mathfrak{X}_{M}\right)$ be the functor of extension by zero. It is the right adjoint of the functor $j^{*}: \operatorname{Sh}\left(\mathfrak{X}_{M}\right) \rightarrow \operatorname{Sh}\left(\mathfrak{U}_{M}\right)$ which is the restriction functor from $\mathfrak{X}_{M}$ to the subcategory $\mathfrak{U}_{M} \subset \mathfrak{X}_{M}$; see Err. Let $f:\left.\mathcal{G}\right|_{\mathfrak{U}_{M}} \rightarrow \mathcal{F}^{\prime}$ be a morphism of sheaves such that $\left(\mathcal{F}^{\prime}, \mathcal{I}^{\prime}, \gamma^{\prime}\right)$ is a $\mathbb{W}(k)$-DP sheaf of algebras on $\mathfrak{U}_{M}$ and $f(\mathcal{J}) \subset \mathcal{I}^{\prime}$. Then $\left(j_{!}\left(\mathcal{F}^{\prime}\right), j_{!}\left(\mathcal{I}^{\prime}\right), j_{!}\left(\gamma^{\prime}\right)\right)$ is a $\mathbb{W}(k)$-DP sheaf of algebras and by adjointness of $j_{\text {! }}$ we get a morphism $j_{!}(f): \mathcal{G} \rightarrow j_{!}\left(\mathcal{F}^{\prime}\right)$. The latter extends uniquely to a morphism $\mathcal{F} \rightarrow j_{!}\left(\mathcal{F}^{\prime}\right)$ of $\mathbb{W}(k)$-DP sheaves of algebras by the universal 
property of $\mathcal{F}$. Restricting to $\mathfrak{U}_{M}$ we get a morphism $\left.\mathcal{F}\right|_{\mathfrak{U}_{M}} \rightarrow \mathcal{F}^{\prime}$ of $\mathbb{W}(k)$-DP sheaves of algebras extending $f$. Using the adjointness of $j_{\text {! }}$ and the universal property of $\mathcal{F}$ one proves that such a morphism is unique. The claim follows.

By lemma 2.14 for every object $(\mathcal{U}, \mathcal{W}) \in \mathfrak{X}_{M}$ we have a natural identification $\mathbb{W}_{n, M}(\mathcal{U}, \mathcal{W}) \cong$ $\beta_{M, \bar{K}, *}\left(\mathbb{W}_{n}(\mathcal{U}, \mathcal{W})\right)^{\operatorname{Gal}(\bar{K} / M)}$. We used the fact that $\beta_{M, \bar{K}}^{-1}\left(\mathbb{W}_{n, M}\right)(\mathcal{U}, \mathcal{W})=\mathbb{W}_{n}(\mathcal{U}, \mathcal{W})$. Define $\mathbb{A}_{\text {cris,n,M }}^{\nabla}$ to be the sheaf on $\mathfrak{X}_{M}$ associated to the pre-sheaf given by

$$
(\mathcal{U}, \mathcal{W}) \mapsto\left(A_{\text {cris }, \mathrm{n}} \otimes_{W_{n}}\left(\mathbb{W}_{n}(\mathcal{U}, \mathcal{W})\right)\right)^{\operatorname{Gal}(\bar{K} / M)} .
$$

Let $\theta_{M, n}: \mathbb{A}_{\text {cris,n,M }}^{\nabla} \longrightarrow \mathcal{O}_{\mathfrak{X}_{M}} / p^{n} \mathcal{O}_{\mathfrak{X}_{M}}$ be the map of sheaves induced by the map of pre-sheaves

$$
\left.\left(A_{\text {cris }, \mathrm{n}} \otimes_{W_{n}}\left(\mathbb{W}_{n}(\mathcal{U}, \mathcal{W})\right)\right)\right)^{\operatorname{Gal}(\bar{K} / M)} \rightarrow\left(\mathcal{O}_{\mathfrak{X}_{M}} / p^{n} \mathcal{O}_{\mathfrak{X}_{M}}\right)(\mathcal{U}, \mathcal{W})
$$

given by $\theta_{n} \otimes \theta_{n, M}$. Here using again lemma 2.14 we have identified

$$
\left(\beta_{M, \bar{K}, *}\left(\mathcal{O}_{\mathfrak{X}_{\bar{K}}} / p^{n} \mathcal{O}_{\mathfrak{X}_{\bar{K}}}\right)(\mathcal{U}, \mathcal{W})\right)^{\operatorname{Gal}(\bar{K} / M)}
$$

with $\left(\mathcal{O}_{\mathfrak{X}_{M}} / p^{n} \mathcal{O}_{\mathfrak{X}_{M}}\right)(\mathcal{U}, \mathcal{W})$. We get from lemma 2.19 that $\operatorname{Ker}\left(\theta_{M, n}\right)(\mathcal{U}, \mathcal{W})$ coincides with the $\operatorname{Gal}(\bar{K} / M)$-invariants of the ideal of $A_{\text {cris,n }} \otimes_{W_{n}} \mathbb{W}_{n}\left(\mathcal{O}_{\mathfrak{x}_{M}}(\mathcal{U}, \mathcal{W}) / p \mathcal{O}_{\mathfrak{X}_{\bar{K}}}(\mathcal{U}, \mathcal{W})\right)$ generated by $\operatorname{Ker}\left(\theta_{n}\right)^{\text {DP }}$. Such an ideal has $\mathbb{W}(k)$-DP structure thanks to corollary 2.22. In particular the sheaf $\operatorname{Ker}\left(\theta_{n, M}\right)$ is endowed with $\mathbb{W}(k)$-DP structure as well. Using the identification $\mathbb{W}_{n, M}(\mathcal{U}, \mathcal{W}) \cong$ $\left(\mathbb{W}_{n}(\mathcal{U}, \mathcal{W})\right)^{\operatorname{Gal}(\bar{K} / M)}$ we also have a natural map

$$
h_{M, n}: \mathbb{W}_{n, M} \longrightarrow \mathbb{A}_{\text {cris,n,M }}^{\nabla}
$$

Since $\mathcal{O}_{\mathfrak{x}_{M}}$ is a sheaf of $\mathcal{O}_{M}$-algebras, $\mathbb{W}_{n, M}$ is a sheaf of $\mathcal{O}_{M_{0}}$-algebras. Consider the map $r_{n+1}: \mathbb{W}_{n+1} \rightarrow \mathbb{W}_{n}$ defined by the natural projection composed with Frobenius. Now we tensor with $A_{\text {cris,n+1 }}$ over $W_{n}$. Since $\xi_{n}$ is the image of $\xi_{n+1}$ via $r_{n+1}$, taking $\operatorname{Gal}(\bar{K} / M)$-invariants, we get a natural map $r_{M, n+1}: \mathbb{A}_{\text {cris,n+1,M }}^{\nabla} \rightarrow \mathbb{A}_{\text {cris,n,M}}^{\nabla}$. Denote by $\mathbb{A}_{\text {cris }, \mathrm{M}}^{\nabla}$ the sheaf in $\operatorname{Sh}\left(\mathfrak{X}_{\bar{K}}\right)^{\mathbb{N}}$ defined by the family $\left\{\mathbb{A}_{\text {cris,n,M }}^{\nabla}\right\}_{n}$ with the transition maps $\left\{r_{M, n+1}\right\}_{n}$.

Proposition 2.24. 1) The sheaf of rings $\mathbb{A}_{\text {cris,n,M }}^{\nabla}$, with the sheaf of ideals $\operatorname{Ker}\left(\theta_{n, M}\right)$ and the natural map $h_{n, M}: \mathbb{W}_{n, M} \longrightarrow \mathbb{A}_{\text {cris,n,M }}^{\nabla}$, is the $\mathbb{W}(k)-D P$ envelope of $\mathbb{W}_{n, M}$ with respect to $K \operatorname{Ker}\left(\theta_{n, M}\right)$.

2) The system of sheaves of rings $\mathbb{A}_{\text {cris,M }}^{\nabla}$, with the sheaf of ideals $\left\{\operatorname{Ker}\left(\theta_{n, M}\right)\right\}_{n}$ and the natural map $h_{M}=\left\{h_{n, M}\right\}_{n}: \mathbb{A}_{\text {inf,M }}^{+} \longrightarrow \mathbb{A}_{\text {cris,M }}^{\nabla}$ is the $\mathbb{W}(k)$-DP envelope of $\mathbb{A}_{\text {inf,M }}^{+}$with respect to $\left\{\operatorname{Ker}\left(\theta_{n, M}\right)\right\}_{n}$.

3) The Frobenius map $\varphi: \mathbb{W}_{n, M} \rightarrow \mathbb{W}_{n, M}$ defines a map $\varphi_{n}: \mathbb{A}_{\text {cris,n,M }}^{\nabla} \rightarrow \mathbb{A}_{\text {cris,n,M }}^{\nabla}$.

4) For varying $n$ the maps $\left\{\varphi_{n}\right\}_{n}$ define a morphism $\varphi: \mathbb{A}_{\text {cris }, M}^{\nabla} \rightarrow \mathbb{A}_{\text {cris,M }}^{\nabla}$ in $S h\left(\mathfrak{X}_{M}\right)^{\mathbb{N}}$;

5) If $M_{1} \subset M_{2}$ is a Galois field extension we have a natural isomorphism $\beta_{M_{1}, M_{2}}^{*}\left(\mathbb{A}_{\text {cris,n, } \mathrm{M}_{1}}^{\nabla}\right) \cong$ $\mathbb{A}_{\text {cris,n, } \mathrm{M}_{2}}^{\nabla}$ of $\mathbb{W}(k)-D P$ sheaves of algebras, compatible with Frobenius and the natural structures of $\mathbb{W}_{n, M_{2}}$-sheaves of modules;

6) We have a natural isomorphism $\beta_{M_{1}, M_{2}}^{*}\left(\mathbb{A}_{\text {cris, } \mathrm{M}_{1}}^{\nabla}\right) \cong \mathbb{A}_{\text {cris, } \mathrm{M}_{2}}^{\nabla}$ of $\mathbb{W}(k)$-DP sheaves of algebras, compatible with Frobenius and the natural structures of $\mathbb{A}_{\mathrm{inf}, \mathrm{M}_{2}}^{+}$-sheaves of modules. 
Proof. (1) Let $\mathcal{G}$ be a $\mathbb{W}(k)$-DP sheaf of algebras and let $f: \mathbb{W}_{n, M} \rightarrow \mathcal{G}$ be a morphism sending $\operatorname{Ker}\left(\theta_{n, M}\right)$ to the DP ideal of $\mathcal{G}$. Due to 2.14, for every $(\mathcal{U}, \mathcal{W}) \in \mathfrak{X}_{M}$, we have $\mathcal{G}(\mathcal{U}, \mathcal{W})=\left(\beta_{M, \bar{K}}^{-1}(\mathcal{G})(\mathcal{U}, \mathcal{W})\right)^{\operatorname{Gal}(\bar{K} / M)}$. Since $\beta_{M, \bar{K}}^{-1}\left(\mathbb{W}_{n, M}\right)(\mathcal{U}, \mathcal{W})$ is equal to $\mathbb{W}_{n}(\mathcal{U}, \mathcal{W})$, the map $\beta_{M, \bar{K}}^{-1}(f)$ extends uniquely to a map $A_{\text {cris,n }} \otimes_{W_{n}} \mathbb{W}_{n}(\mathcal{U}, \mathcal{W}) \rightarrow \beta_{M, \bar{K}}^{-1}(\mathcal{G})(\mathcal{U}, \mathcal{W})$. Taking $\operatorname{Gal}(\bar{K} / M)$-invariants and using the definition of $\mathbb{A}_{\text {cris,n,M }}^{\nabla}$, we get a unique map $\mathbb{A}_{\text {cris,n,M }}^{\nabla} \rightarrow \mathcal{G}$ as $\mathbb{W}(k)$-DP sheaves of algebras extending $f$. This proves the universal property of $\mathbb{A}_{\text {cris,n,M}}^{\nabla}$. Claim (2) follows from claim (1). Recall that Frobenius on $W_{n}$ extends to an operator $\varphi$ on $A_{\text {cris,n }}$. Claims (3) and (4) follow. (5) The existence of a natural map $\beta_{M_{1}, M_{2}}^{*}\left(\mathbb{A}_{\text {cris,n, } \mathrm{M}_{1}}^{\nabla}\right) \rightarrow \mathbb{A}_{\text {cris,n, } \mathrm{M}_{2}}^{\nabla}$, compatible with Frobenius and the structure of $\mathbb{W}_{n, M_{2}}$-modules follows from the definition of $\mathbb{A}_{\text {cris,n }}^{\nabla}$. To check that it is an isomorphism it suffices to prove it for the stalks. The stalk of $\beta_{M_{1}, M_{2}}^{-1}\left(\mathbb{W}_{n, M_{1}}\right)$ at a point $x \in X$ is $\mathbb{W}_{n}\left(\mathcal{O}_{\mathfrak{X}_{M_{1}}, x} / p \mathcal{O}_{\mathfrak{X}_{M_{1}}, x}\right)$; see [AI, Prop. 4.4]. The latter is a $W_{n}-$ algebra. It then follows from 2.19 that the stalk $\mathbb{A}_{\text {cris,n, }, \mathrm{M}_{1}, \mathrm{x}}$ is $A_{\text {cris,n }} \otimes_{W_{n}} \mathbb{W}_{n}\left(\mathcal{O}_{\mathfrak{X}_{M_{1}}, x} / p \mathcal{O}_{\mathfrak{X}_{M_{2}}, x}\right)$. Since $\mathcal{O}_{\mathfrak{X}_{M_{1}}, x} \cong \mathcal{O}_{\mathfrak{X}_{M_{2}}, x}$, the claim follows. Claim (6) follows from (5)

The next step is to study the localization $\mathbb{A}_{\text {cris, M }}^{\nabla}$ over small affines. In analogy with the classical case of $A_{\text {cris }}$ recalled in $\$ 1.2$ we provide a second essentially equivalent definition of $\mathbb{A}_{\text {cris }, \mathrm{M}}^{\nabla}$ via the system $\mathbb{A}_{\text {cris,M }}^{\nabla} / p^{n} \mathbb{A}_{\text {cris,M }}^{\nabla}$ for varying $n \in \mathbb{N}$. Let $\mathbb{A}_{\text {cris }, \mathrm{n}, \mathrm{M}}^{\prime} \nabla$ be the sheaf on $\mathfrak{X}_{M}$ associated to the pre-sheaf given by

$$
(\mathcal{U}, \mathcal{W}) \mapsto\left(\left(A_{\text {cris }} / p^{n} A_{\text {cris }}\right) \otimes_{W_{n}} \mathbb{W}_{n}(\mathcal{U}, \mathcal{W})\right)^{\operatorname{Gal}(\bar{K} / M)}
$$

Let $\theta_{M, n}^{\prime}: \mathbb{A}_{\text {cris,n,M }}^{\prime} \nabla \mathcal{O}_{\mathfrak{X}_{M}} / p^{n} \mathcal{O}_{\mathfrak{X}_{M}}$ be the map $\theta_{M, n} \circ\left(q_{n} \otimes \varphi\right)$ (we refer to $\$ 1.2$ for the map $\left.q_{n}: A_{\text {cris }} / p^{n} A_{\text {cris }} \rightarrow A_{\text {cris }, \mathrm{n}}\right)$. Denote by $\mathbb{A}_{\text {cris,M }}^{\prime}$ the sheaf in $\operatorname{Sh}\left(\mathfrak{X}_{M}\right)^{\mathbb{N}}$ defined by the family $\left\{\mathbb{A}_{\text {cris,n,M }}^{\prime}\right\}$ with the transition maps $r_{M, n+1}^{\prime}: \mathbb{A}_{\text {cris }, \mathrm{n}+1, \mathrm{M}}^{\prime} \rightarrow \mathbb{A}_{\text {cris,n,M }}^{\prime} \nabla$ induced by $r_{n+1}: \mathbb{W}_{n+1, M} \rightarrow$ $\mathbb{W}_{n, M}$. For every $n \in \mathbb{N}$ define the map of sheaves

$$
q_{M, n}: \mathbb{A}_{\text {cris }, \mathrm{n}, \mathrm{M}}^{\prime} \longrightarrow \mathbb{A}_{\text {cris }, \mathrm{n}, \mathrm{M}}^{\nabla}
$$

associated to the map of pre-sheaves inducing $q_{n}: A_{\text {cris }} / p^{n} A_{\text {cris }} \rightarrow A_{\text {cris,n }}$ and Frobenius on $\mathbb{W}_{n, M}(\mathcal{U}, \mathcal{W})$. Consider the map of sheaves

$$
u_{n, M}: \mathbb{A}_{\text {cris,n+1,M }}^{\nabla} \longrightarrow \mathbb{A}_{\text {cris }, \mathrm{n}, \mathrm{M}}^{\prime}
$$

associated to the map of pre-sheaves which induces $u_{n}: A_{\text {cris,n+1 }} \rightarrow A_{\text {cris }} / p^{n} A_{\text {cris }}$ (see 1.2) and the natural projection $\mathbb{W}_{n+1, M}(\mathcal{U}, \mathcal{W}) \rightarrow \mathbb{W}_{n, M}(\mathcal{U}, \mathcal{W})$

Proposition 2.25. 1) The sheaf of rings $\mathbb{A}_{\text {cris,n,M }}^{\prime} \nabla$ with the sheaf of ideals $\operatorname{Ker}\left(\theta_{n, M}^{\prime}\right)$ and the natural map $h_{n, M}^{\prime}: \mathbb{W}_{n, M} \longrightarrow \mathbb{A}_{\text {cris,n,M }}^{\prime \nabla}$ is the $\mathbb{W}(k)-D P$ envelope of $\mathbb{W}_{n, M}$ with respect to $\operatorname{Ker}\left(\theta_{n, M} \circ \varphi\right)$.

2) The system of sheaves of rings $\mathbb{A}_{\text {cris, },}^{\prime} \nabla$, with the sheaf of ideals $\left\{\operatorname{Ker}\left(\theta_{n, M}^{\prime}\right)\right\}_{n}$ and the natural map $h_{M}^{\prime}=\left\{h_{n, M}^{\prime}\right\}_{n}: \mathbb{A}_{\text {inf,M }}^{+} \longrightarrow \mathbb{A}_{\text {cris,M }}^{\prime}$ is the $\mathbb{W}(k)$-DP envelope of $\mathbb{A}_{\text {inf,M }}^{+}$with respect to $\left\{\operatorname{Ker}\left(\theta_{n, M} \circ \varphi\right)\right\}_{n}$.

3) Frobenius on $\mathbb{W}_{n, M}$ defines maps $\varphi_{n}^{\prime}: \mathbb{A}_{\text {cris,n,M }}^{\prime} \rightarrow \mathbb{A}_{\text {cris,n,M }}^{\prime} \nabla$ which are compatible for varying $n$ and give a morphism $\varphi:=\left\{\varphi_{n}^{\prime}\right\}: \mathbb{A}_{\text {cris, }, \mathrm{M}}^{\prime} \rightarrow \mathbb{A}_{\text {cris }, \mathrm{M}}^{\prime} \nabla$ 
4) For every $n \in \mathbb{N}$ we have $q_{M, n} \circ u_{M, n}=r_{M, n+1}$ and $u_{M, n} \circ q_{M, n+1}=r_{M, n+1}^{\prime}$. Furthermore, the following diagrams commute

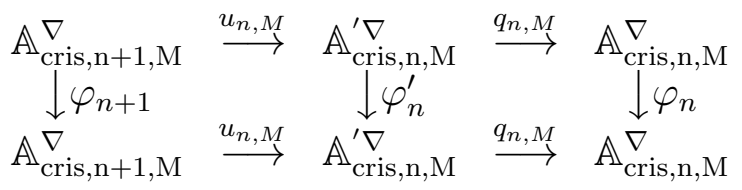

and

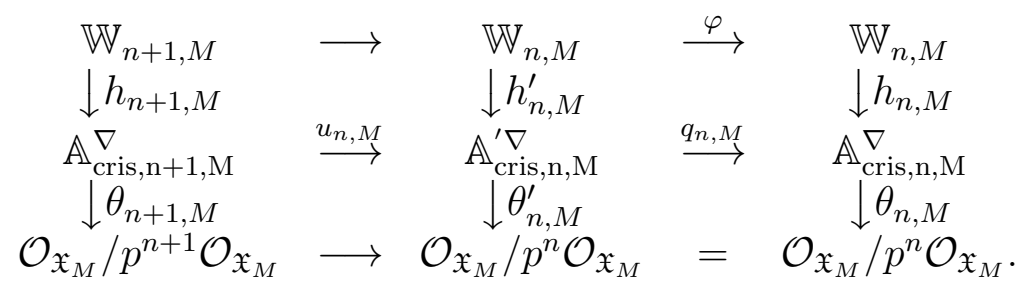

5) If $M_{1} \subset M_{2}$ is a Galois field extension we have a natural isomorphism $\beta_{M_{1}, M_{2}}^{*}\left(\mathbb{A}_{\text {cris,n, } \mathrm{M}_{1}}^{\prime}\right) \cong$ $\mathbb{A}_{\text {cris,n, } \mathrm{M}_{2}}^{\prime}$ of $\mathbb{W}(k)$-DP sheaves of algebras, compatible with Frobenius and the natural structures of $\mathbb{W}_{n, M_{2}}$-sheaves of modules;

6) We have a natural isomorphism $\beta_{M_{1}, M_{2}}^{*}\left(\mathbb{A}_{\text {cris, } \mathrm{M}_{1}}^{\prime}\right) \cong \mathbb{A}_{\text {cris, } \mathrm{M}_{2}}^{\prime}$ of $\mathbb{W}(k)$-DP sheaves of algebras, compatible with Frobenius and the natural structures of $\mathbb{A}_{\mathrm{inf}, \mathrm{M}_{2}}^{+}$-sheaves of modules.

Write $q_{M}:=\left\{q_{n, M}\right\}_{n}: \mathbb{A}_{\text {cris }, \mathrm{M}}^{\prime} \rightarrow \mathbb{A}_{\text {cris,M }}^{\nabla}$ and $u_{M}:=\left\{u_{n, M}\right\}_{n}: \mathbb{A}_{\text {cris }, \mathrm{M}}^{\nabla} \rightarrow \mathbb{A}_{\text {cris }, \mathrm{M}}^{\prime} \nabla$

Lemma 2.26. a) For every $n$ we have an exact sequence

$$
0 \longrightarrow \mathbb{A}_{\text {cris }, \mathrm{n}, \mathrm{M}}^{\prime} \stackrel{a}{\longrightarrow} \mathbb{A}_{\text {cris }, \mathrm{n}+1, \mathrm{M}}^{\prime} \stackrel{b}{\longrightarrow} \mathbb{A}_{\text {cris }, 1, \mathrm{M}}^{\prime} \longrightarrow 0
$$

where $b=r_{2, M}^{\prime} \circ \cdots \circ r_{n, M}^{\prime} \circ r_{n+1, M}^{\prime}$ and $a$ is the map of sheaves associated to the Vershiebung $\mathbb{V}: \mathbb{W}_{n, M} \longrightarrow \mathbb{W}_{n+1, M}$

b) We have $\mathbb{A}_{\text {cris }, 1, \overline{\mathrm{K}}}^{\prime \nabla}=\mathcal{O}_{\mathfrak{X}_{\bar{K}}} / p \mathcal{O}_{\mathfrak{X}_{\bar{K}}}\left[\delta_{0}, \delta_{1}, \ldots\right] /\left(\delta_{m}^{p}\right)_{m \geq 0}$.

c) We have $\mathbb{A}_{\text {cris }, \mathrm{n}, \overline{\mathrm{K}}}^{\prime}(\mathcal{U}, \mathcal{W})=\left(A_{\text {cris }} / p^{n} A_{\text {cris }}\right) \otimes_{W_{n}}\left(\mathbb{W}_{n, \bar{K}}(\mathcal{U}, \mathcal{W})\right)$ for every $(\mathcal{U}, \mathcal{W}) \in \mathfrak{X}_{\bar{K}}$. In particular the sequence in (a) is exact also as sequence of presheaves for $M=\bar{K}$.

Proof. a) Certainly $b \circ a=0$. To check exactness we study the stalks. Since for any sheaf $\mathcal{G}$ on $\mathfrak{X}_{M}$ we have $\beta_{M, \bar{K}}^{*}(\mathcal{G})_{x}=\mathcal{G}_{x}$ by [AI, Prop. 4.4] and since $\beta_{M, \bar{K}}^{*}\left(\mathbb{A}_{\text {cris }, \mathrm{n}, \mathrm{M}}^{\prime}\right) \cong \mathbb{A}_{\text {cris, } \mathrm{n}, \overline{\mathrm{K}}}^{\prime \nabla}$ by proposition 2.25 it suffices to prove the claim for $M=\bar{K}$. The kernel $H$ of the natural projection $s: \mathbb{W}_{n+1} \rightarrow \mathbb{W}_{1}=\mathcal{O}_{\mathfrak{X}_{\bar{K}}} / p \mathcal{O}_{\mathfrak{X}_{\bar{K}}}$ is identified with $\mathbb{W}_{n}$ via Vershiebung. It is a $W_{n+1}$-module via the projection $W_{n+1} \rightarrow W_{n}$ composed with Frobenius on $W_{n}$ and the structure of $W_{n}$-module of $\mathbb{W}_{n}$. Hence

$$
\left(A_{\text {cris }} / p^{n+1} A_{\text {cris }}\right) \otimes H \cong\left(A_{\text {cris }} / p^{n+1} A_{\text {cris }}\right) \otimes \mathbb{W}_{n},
$$

where $\otimes$ stands for $\otimes_{W_{n+1}}$. Since $s\left(\xi_{n+2}^{p}\right) \equiv p^{\frac{1}{p^{n}}}$ we have

$$
\left(A_{\text {cris }} / p^{n+1} A_{\text {cris }}\right) \otimes_{W_{n+1}} \mathbb{W}_{1} \cong \mathcal{O}_{\mathfrak{X}_{\bar{K}}} / p^{\frac{1}{p^{n}}} \mathcal{O}_{\mathfrak{X}_{\bar{K}}}\left[\delta_{0}, \delta_{1}, \ldots\right] /\left(\delta_{m}^{p}\right)_{m \geq 0} .
$$


Recall from 2.22 that Frobenius to the $n$-th power $\varphi^{n}$ gives an isomorphism $\mathcal{O}_{\mathfrak{X}_{\bar{K}}} / p^{1 / p^{n}} \mathcal{O}_{\mathfrak{X}_{\bar{K}}} \rightarrow$ $\mathcal{O}_{\mathfrak{X}_{\bar{K}}} / p \mathcal{O}_{\mathfrak{X}_{\bar{K}}}$. Hence

$$
\left(A_{\text {cris }} / p^{n+1} A_{\text {cris }}\right) \otimes_{W_{n+1}}\left(\mathcal{O}_{\mathfrak{X}_{\bar{K}}} / p \mathcal{O}_{\mathfrak{X}_{\bar{K}}}\right) \cong \mathcal{O}_{\mathfrak{X}_{\bar{K}}} / p \mathcal{O}_{\mathfrak{X}_{\bar{K}}}\left[\delta_{0}, \delta_{1}, \ldots\right] /\left(\delta_{m}^{p}\right)_{m \geq 0} .
$$

The composite of $s$ with $\varphi^{n}$ is $r_{2} \circ \cdots \circ r_{n} \circ r_{n+1}: \mathbb{W}_{n+1} \longrightarrow \mathcal{O}_{\mathfrak{X}_{\bar{K}}} / p \mathcal{O}_{\mathfrak{X}_{\bar{K}}}$. This proves the exactness of the sequence displayed in Claim a) with the exception of the exactness on the left. We prove the left exactness on stalks. Let $x$ be a point of $X$. Note that $\xi=[\widetilde{p}]-p$. Since the ideal generated by $p$ admits $\mathbb{W}(k)-\mathrm{DP}$ in $A_{\text {cris }}$, the $\mathbb{W}(k)$-DP envelope of $\xi$ in $A_{\text {cris }}$ coincides with the $\mathbb{W}(k)$-DP envelope of $[\tilde{p}]$ in $A_{\text {cris }}$ i. e.,

$$
A_{\text {cris }} / p^{n} A_{\text {cris }} \cong W_{n}\left\langle\widetilde{p}_{n+1}\right\rangle=W_{n}\left[\delta_{0}, \delta_{1}, \ldots\right] /\left(p \delta_{0}-\widetilde{p}_{n+1}^{p}, p \delta_{m+1}-\delta_{m}^{p}\right)_{m \geq 0} .
$$

Define $B:=\mathbb{W}_{n}\left(\mathcal{O}_{\mathfrak{X}_{\bar{K}}, x} / p \mathcal{O}_{\mathfrak{X}_{\bar{K}}, x}\right)\left[\delta_{0}, \delta_{1}, \ldots\right] /\left(p \delta_{m+1}-\delta_{m}^{p}\right)_{m \geq 0}$. Similarly, denote by

$$
C:=\mathbb{W}_{n+1}\left(\mathcal{O}_{\mathfrak{X}_{\bar{K}}, x} / p \mathcal{O}_{\mathfrak{X}_{\bar{K}}, x}\right)\left[\delta_{0}, \delta_{1}, \ldots\right] /\left(p \delta_{m+1}-\delta_{m}^{p}\right)_{m \geq 0}
$$

and write $D:=\mathcal{O}_{\mathfrak{X}_{\bar{K}}, x} / p \mathcal{O}_{\mathfrak{X}_{\bar{K}}, x}\left[\delta_{0}, \delta_{1}, \ldots\right] /\left(\delta_{m}^{p}\right)_{m \geq 0}$. Note that $B /\left(p \delta_{0}-\widetilde{p}_{n+1}^{p}\right) B$ is the stalk $\mathbb{A}_{\text {cris }, \mathrm{n}, \overline{\mathrm{K}}, \mathrm{x}}^{\prime}$ of $\mathbb{A}_{\text {cris }, \mathrm{n}, \overline{\mathrm{K}}}^{\prime}$ at $x, C /\left(p \delta_{0}-\widetilde{p}_{n+2}^{p}\right) C$ is $\mathbb{A}_{\text {cris }, \overline{\mathrm{K}}, \mathrm{n}+1, \mathrm{x}}^{\prime}$ and $D / \widetilde{p}_{n+2}^{p} D=\mathbb{A}_{\text {cris }, 1, \overline{\mathrm{K}}, \mathrm{x}}^{\prime}$. We have the following commutative diagram:

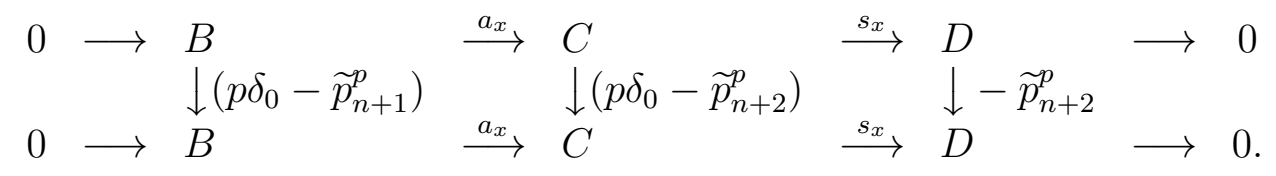

Here, $a_{x}$ sends $\delta_{i} \mapsto \delta_{i}$ and induces Vershiebung $\mathbb{W}_{n}\left(\mathcal{O}_{\mathfrak{x}_{\bar{K}}, x} / p \mathcal{O}_{\mathfrak{X}_{\bar{K}}, x}\right) \rightarrow \mathbb{W}_{n+1}\left(\mathcal{O}_{\mathfrak{X}_{\bar{K}}, x} / p \mathcal{O}_{\mathfrak{x}_{\bar{K}}, x}\right)$. Since $B$ (resp. $C$ ) is a free $\mathbb{W}_{n}\left(\mathcal{O}_{\mathfrak{X}_{\bar{K}}, x} / p \mathcal{O}_{\mathfrak{X}_{\bar{K}}, x}\right)$-module (resp. $\mathbb{W}_{n+1}\left(\mathcal{O}_{\mathfrak{X}_{\bar{K}}, x} / p \mathcal{O}_{\mathfrak{X}_{\bar{K}}, x}\right)$-module) with basis given by the monomials in the $\delta_{i}$ 's and Vershiebung is injective the map $a_{x}$ is injective. The map $s_{x}$ is the natural projection. Since also $D$ is a free $\mathcal{O}_{\mathfrak{X}_{\bar{K}}, x} / p \mathcal{O}_{\mathfrak{X}_{\bar{K}}, x}$-module with basis given by the monomials in the $\delta_{i}$ 's the rows in the displayed diagram are exact. The sequence of cokernels $B /\left(p \delta_{0}-\widetilde{p}_{n+1}^{p}\right) B \rightarrow C /\left(p \delta_{0}-\widetilde{p}_{n+2}^{p}\right) C$ is the map on stalks associated to $a$. Note that $\widetilde{p}_{n+1}=\widetilde{p}_{n+2}^{p}=p^{1 / p^{n}}$ in $\mathcal{O}_{\bar{K}} / p \mathcal{O}_{\bar{K}}$ and the kernel of multiplication by $p^{1 / p^{n}}$ on $D$ is $p p^{-1 / p^{n}} D=$ $p^{\frac{p^{n}-1}{p^{n}}} D=\widetilde{p}_{n+1}^{p^{n}-1} D$. Choose $y \in D$ and let $x \in C$ be the lift defined taking the Teichmüller lifts of the coefficients of $x$ with respect to the $\mathcal{O}_{\mathfrak{X}_{\bar{K}}, x} / p \mathcal{O}_{\mathfrak{X}_{\bar{K}}, x}$-basis of $D$ given by the monomials in the $\delta_{i}$ 's. In particular $\widetilde{p}_{n+1}^{p^{n}} y=\widetilde{p} y=0$. Put $z:=\sum_{i=0}^{p^{n}-1} p^{i} \delta_{0}^{i} \widetilde{p}_{n+1}^{p^{n}-i-1} y$. Then

$$
\left(p \delta_{0}-\widetilde{p}_{n+1}\right) z=\sum_{i=0}^{p^{n}-1} \delta_{0}^{i+1} p^{i+1} \widetilde{p}_{n+1}^{p^{n}-i-1} y-\sum_{i=0}^{p^{n}-1} \delta_{0}^{i} p^{i} \widetilde{p}_{n+1}^{p^{n}-i} y=\delta_{0}^{p^{n}} p^{p^{n}} y-\widetilde{p}_{n+1}^{p^{n}} y=0
$$

and $s_{x}(z)=p^{\frac{p^{n}-1}{p^{n}}} y$. This proves that the kernel of multiplication by $p \delta_{0}-\widetilde{p}_{n+2}^{p}$ on $C$ surjects onto the kernel of multiplication by $\widetilde{p}_{n+2}^{p}$ on $D$. The claimed left exactness follows from this using the snake lemma in the displayed diagram.

b) follows using that $A_{\text {cris }} / p A_{\text {cris }} \cong \mathcal{O}_{\bar{K}} / p \mathcal{O}_{\bar{K}}\left[\delta_{0}, \delta_{1}, \ldots\right] /\left(\delta_{m}^{p}\right)_{m \geq 0}$; see 1.2 . 
c) We prove the claim by induction on $n$. For $n=1$ it follows from (b) since $\mathbb{A}_{\text {cris }, 1, \overline{\mathrm{K}}}^{\prime}$ is a direct sum of copies of $\mathcal{O}_{\mathfrak{X}_{\bar{K}}} / p \mathcal{O}_{\mathfrak{X}_{\bar{K}}}$. Suppose the claim proved for $n$. Write $\underline{A}_{n}^{\prime}$ for the presheaf $(\mathcal{U}, \mathcal{W}) \mapsto\left(A_{\text {cris }} / p^{n} A_{\text {cris }}\right) \otimes_{W_{n}}\left(\mathbb{W}_{n}(\mathcal{U}, \mathcal{W})\right)$. Consider the following commutative diagram:

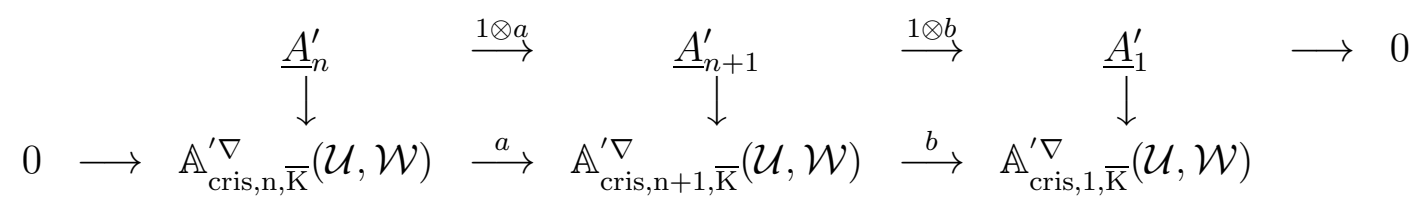

The bottom row is exact due to (a). The top one is exact as well (see the proof of (a)). This fact together with the inductive hypothesis and a diagram chase imply the claim.

To conclude the comparison between $\mathbb{A}_{\text {cris,n,M }}^{\prime} \nabla$ and $\mathbb{A}_{\text {cris,n,M }}^{\nabla}$ we prove the following:

Lemma 2.27. For positive integers $m>n$ the map $u_{n, M} \circ r_{n+2, M} \circ \cdots \circ r_{m, M}: \mathbb{A}_{\text {cris,m,M }}^{\nabla} \longrightarrow$ $\mathbb{A}_{\text {cris }, \mathrm{n}, \mathrm{M}}^{\prime}$ induces an isomorphism $\mathbb{A}_{\text {cris }, \mathrm{m}, \mathrm{M}}^{\nabla} / p^{n} \mathbb{A}_{\text {cris }, \mathrm{m}, \mathrm{M}}^{\nabla} \longrightarrow \mathbb{A}_{\text {cris }, \mathrm{n}, \mathrm{M}}^{\prime}{ }^{\prime}$

Proof. As in the proof of lemma 2.26(b) it suffices to prove the lemma for $M=\bar{K}$. We can write multiplication by $p^{n}$ on $\mathbb{W}_{m}$ as the composite of $\mathbb{V}^{n} \circ \varphi^{n}$ with $\mathbb{V}=$ Vershiebung and $\varphi=$ Frobenius. Since $\varphi$ is surjective on $\mathcal{O}_{\mathfrak{X}_{\bar{K}}} / p \mathcal{O}_{\mathfrak{X}_{\bar{K}}}$ by $[\mathrm{AI}$, Lemma $4.3(\mathrm{v})]$ we deduce that $\mathbb{W}_{m} / p^{n} \mathbb{W}_{m} \cong \mathbb{W}_{n}$ where the map is the natural projection. Via this identification the map $u_{n} \circ r_{n+1} \circ \cdots \circ$ $r_{m}: \mathbb{W}_{n} \longrightarrow \mathbb{W}_{n}$ is $\varphi^{m-n-1}$ and, hence, sends $\xi_{m} \mapsto \xi_{n+1}$. Note that $\widetilde{p}_{m}=\xi_{m}+p$. Since $p$ and $\xi_{m}$ admit DP in $A_{\text {cris }, \mathrm{m}}\left(\mathcal{O}_{\bar{K}}\right)$ also $\widetilde{p}_{m}$ admits DP. We compute $\mathbb{V}^{s}\left(\widetilde{p}_{m}\right)^{p^{n}}=\left(\varphi^{s}\left(V^{s}\left(\widetilde{p}_{m}\right)\right)\right)^{p^{n-s}}=$ $\left(p^{s} \widetilde{p}_{m}\right)^{p^{n-s}}=p^{s p^{n-s}} \widetilde{p}_{m}^{p^{n-s}}=p^{s p^{n-s}} p^{n-s} ! \widetilde{p}_{m}^{\left[p^{n-s}\right]}$. This is 0 in $A_{\text {cris } \mathrm{m}} / p^{n} A_{\text {cris, } \mathrm{m}}$ since $s p^{n-s}+n-s \geq n$. The element $\widetilde{p}_{m}^{p^{n}}$ generates the kernel of $\varphi^{m-n-1}$ on $\mathcal{O}_{\mathfrak{X}} / p \mathcal{O}_{\mathfrak{X}}$; see the proof of 2.26 . Hence $\left(\mathbb{V}^{s}\left(\widetilde{p}_{m}\right)^{p^{n}}\right)_{0 \leq s \leq n}$ generates the kernel of $\varphi^{m-n-1}$ on $\mathbb{W}_{n}$. Similarly $\left.W_{m} / p^{n} W_{m}\right) \cong W_{n}$ and $\left(\mathbb{V}^{s}\left(\widetilde{p}_{m}\right)^{p^{n}}\right)_{0 \leq s \leq n}$ is the kernel of $\varphi^{m-n-1}$ on $W_{n}$. Hence $A_{\text {cris, } \mathrm{m}} / p^{n} A_{\text {cris }, \mathrm{m}}$ is the DP envelope of $W_{n}$ with respect to $\varphi^{m-n-1}\left(\xi_{m}\right)=\xi_{n+1}$ i. e. it coincides with $A_{\text {cris }} / p^{n} A_{\text {cris }}$. We conclude that $p^{n} \mathbb{A}_{\text {cris }, \mathrm{m}, \overline{\mathrm{K}}}^{\nabla}$ contains the kernel of the map $u_{n, \bar{K}} \circ r_{n+1, \bar{K}} \circ \cdots \circ r_{m, \bar{K}}: \mathbb{A}_{\text {cris }, \mathrm{m}, \overline{\mathrm{K}}}^{\nabla} \longrightarrow \mathbb{A}_{\text {cris, }, \overline{\mathrm{K}}}^{\prime}$. It is also clearly contained in this kernel. The claim follows.

Let $\mathcal{U}=\operatorname{Spf}\left(R_{\mathcal{U}}\right)$ be an object in $X^{\text {et }}$. Assume it is small in the sense of 92.2 . As in $\$ 2.3$ fix a geometric generic point and define $\bar{R}_{\mathcal{U}}$ as in loc. cit. Following [Bri, §6] define $A_{\text {cris }}^{\nabla}\left(\bar{R}_{\mathcal{U}}\right)$ as the $p$-adic completion of the $\mathbb{W}(k)$-DP envelope of $\mathbb{W}\left(\mathcal{R}\left(\bar{R}_{\mathcal{U}}\right)\right)$ with respect to the kernel of the map $\vartheta$ defined as follows. For every $n$ let $\vartheta_{n}$ be the composite of the projection $\mathbb{W}\left(\mathcal{R}\left(\bar{R}_{\mathcal{U}}\right)\right) \rightarrow \mathbb{W}_{n}\left(\mathcal{R}\left(\bar{R}_{\mathcal{U}}\right)\right)$, of the map $\mathbb{W}_{n}\left(\mathcal{R}\left(\bar{R}_{\mathcal{U}}\right)\right) \rightarrow \mathbb{W}_{n}\left(\bar{R}_{\mathcal{U}} / p \bar{R}_{\mathcal{U}}\right)$ associated to the projection $\mathcal{R}\left(\bar{R}_{\mathcal{U}}\right)=\lim _{\leftarrow} \bar{R}_{\mathcal{U}} / p \bar{R}_{\mathcal{U}} \rightarrow \bar{R}_{\mathcal{U}} / p \bar{R}_{\mathcal{U}}$ on the $n$-th component and of $\theta_{n}: \mathbb{W}_{n}\left(\bar{R}_{\mathcal{U}} / p \bar{R}_{\mathcal{U}}\right) \rightarrow$ $\bar{R}_{\mathcal{U}} / p^{n} \bar{R}_{\mathcal{U}}$. Let $\vartheta: \mathbb{W}\left(\mathcal{R}\left(\bar{R}_{\mathcal{U}}\right)\right) \rightarrow \widehat{\bar{R}}_{\mathcal{U}}$ be the map $x \mapsto \lim _{\infty \leftarrow n} \vartheta_{n}(x)$. It is proven in loc. cit. that $\operatorname{Ker}(\vartheta)$ is a principal ideal generated by $\xi$ and that Frobenius on $\mathbb{W}\left(\mathcal{R}\left(\bar{R}_{\mathcal{U}}\right)\right)$ induces Frobenius $\varphi$ on $A_{\text {cris }}^{\nabla}\left(\bar{R}_{\mathcal{U}}\right)$. For every $n$ let $g_{n}$ be the composite of the projection $\mathbb{W}\left(\mathcal{R}\left(\bar{R}_{\mathcal{U}}\right)\right) \rightarrow$ $\mathbb{W}_{n}\left(\mathcal{R}\left(\bar{R}_{\mathcal{U}}\right)\right)$ and of the map $v_{n}: \mathbb{W}_{n}\left(\mathcal{R}\left(\bar{R}_{\mathcal{U}}\right)\right) \rightarrow \mathbb{W}_{n}\left(\bar{R}_{\mathcal{U}} / \bar{R}_{\mathcal{U}}\right)$ associated to the projection $\mathcal{R}\left(\bar{R}_{\mathcal{U}}\right)=\lim _{\leftarrow} \bar{R}_{\mathcal{U}} / p \bar{R}_{\mathcal{U}} \rightarrow \bar{R}_{\mathcal{U}} / p \bar{R}_{\mathcal{U}}$ on the $n+1$-th component. We get that

$$
A_{\text {cris }}^{\nabla}\left(\bar{R}_{\mathcal{U}}\right) / p^{n} A_{\text {cris }}^{\nabla}\left(\bar{R}_{\mathcal{U}}\right) \cong \mathbb{W}_{n}\left(\mathcal{R}\left(\bar{R}_{\mathcal{U}}\right)\right)\left[\delta_{0}, \delta_{1}, \ldots\right] /\left(p \delta_{0}-\xi^{p}, p \delta_{i+1}-\delta_{i}^{p}\right)_{i \geq 0}
$$


Since $g_{n}(\xi)=\xi_{n+1}$, we have a map $g_{n}: A_{\text {cris }}^{\nabla}\left(\bar{R}_{\mathcal{U}}\right) / p^{n} A_{\text {cris }}^{\nabla}\left(\bar{R}_{\mathcal{U}}\right) \longrightarrow \mathbb{A}_{\text {cris,n, }, \overline{\mathrm{K}}^{\prime}}^{\prime}\left(\bar{R}_{\mathcal{U}}\right)$. Note that

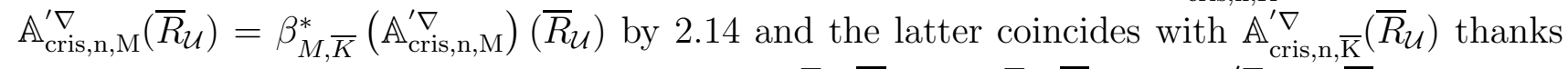
to proposition 2.25 . We then get a map $\left.g_{M, n}: A_{\text {cris }}^{\nabla}\left(\bar{R}_{\mathcal{U}}\right) / p^{n} A_{\text {cris }}^{\nabla}\left(\bar{R}_{\mathcal{U}}\right) \longrightarrow \mathbb{A}_{\text {cris,n,M }}^{\prime} \nabla_{\mathcal{U}}\right)$.

Proposition 2.28. 1) The map $\left.\mathbb{A}_{\text {cris,M }}^{\prime} \nabla_{\mathcal{U}}\right) \rightarrow \mathbb{A}_{\text {cris, },{ }^{\prime}}^{\nabla}\left(\bar{R}_{\mathcal{U}}\right)$ defined by $q_{M}$ is an isomorphism.

2) For every $n \in \mathbb{N}$ the map $g_{n, M}: A_{\text {cris }}^{\nabla}\left(\bar{R}_{\mathcal{U}}\right) / p^{n} A_{\text {cris }}^{\nabla}\left(\bar{R}_{\mathcal{U}}\right) \longrightarrow \mathbb{A}_{\text {cris,n,M }}^{\prime \nabla}\left(\bar{R}_{\mathcal{U}}\right)$ is injective, commutes with the Frobenius maps and its cokernel is annihilated by any element of $\mathbb{I}$.

3) The induced map $A_{\text {cris }}^{\nabla}\left(\bar{R}_{\mathcal{U}}\right) \rightarrow \mathbb{A}_{\text {cris, } \mathrm{M}}^{\nabla}\left(\bar{R}_{\mathcal{U}}\right)$ is an isomorphism and commutes with Frobenius.

Proof. (1) It follows from proposition 2.25(3) that $r_{M}$ defines an inverse. Claims (2) and (3) follow from the next lemma.

Lemma 2.29. For every $n \in \mathbb{N}$ the map $A_{\text {cris }}^{\nabla}\left(\bar{R}_{\mathcal{U}}\right) / p^{n} A_{\text {cris }}^{\nabla}\left(\bar{R}_{\mathcal{U}}\right) \rightarrow \mathbb{A}_{\text {cris, }, \overline{\mathrm{K}}, \mathrm{n}}^{\prime}\left(\bar{R}_{\mathcal{U}}\right)$ is injective, its cokernel is annihilated by any element of $\mathbb{I}$ and the transition map $\mathbb{A}_{\text {cris, }, \overline{\mathrm{K}}, \mathrm{n}+1}^{\prime}\left(\bar{R}_{\mathcal{U}}\right) \rightarrow \mathbb{A}_{\text {cris, }, \overline{\mathrm{K}}_{\mathrm{n}} \mathrm{n}}\left(\bar{R}_{\mathcal{U}}\right)$ factors via $A_{\text {cris }}^{\nabla}\left(\bar{R}_{\mathcal{U}}\right) / p^{n} A_{\text {cris }}^{\nabla}\left(\bar{R}_{\mathcal{U}}\right)$.

Proof. Since $\bar{R}_{\mathcal{U}}$ is a normal ring and Frobenius is surjective on $\bar{R}_{\mathcal{U}} / p \bar{R}_{\mathcal{U}}$ by [Bri, Prop. 2.0.1], the kernel of the projection $\mathcal{R}\left(\bar{R}_{\mathcal{U}}\right)=\lim _{\leftarrow} \bar{R}_{\mathcal{U}} / p \bar{R}_{\mathcal{U}} \rightarrow \bar{R}_{\mathcal{U}} / p \bar{R}_{\mathcal{U}}$ on the $n+1$-factor is $\widetilde{p}^{p^{n}}$. Since $\xi=\widetilde{p}-p$ and both $p$ and $\xi$ have DP in $A_{\text {cris }}^{\nabla}\left(\bar{R}_{\mathcal{U}}\right)$, also $\widetilde{p}$ admits DP. As in the proof of lemma 2.27 it follows that $\mathbb{V}^{s}([\widetilde{p}])^{p^{n}} \equiv 0$ in $A_{\text {cris }}^{\nabla}\left(\bar{R}_{\mathcal{U}}\right) / p^{n} A_{\text {cris }}^{\nabla}\left(\bar{R}_{\mathcal{U}}\right)$. These elements generate the kernel of $v_{n}$. Hence

$$
A_{\text {cris }}^{\nabla}\left(\bar{R}_{\mathcal{U}}\right) / p^{n} A_{\text {cris }}^{\nabla}\left(\bar{R}_{\mathcal{U}}\right) \cong \mathbb{W}_{n}\left(\bar{R}_{\mathcal{U}} / p \bar{R}_{\mathcal{U}}\right)\left[\delta_{0}, \delta_{1}, \ldots\right] /\left(p \delta_{0}-\xi^{p}, p \delta_{i+1}-\delta_{i}^{p}\right)_{i \geq 0}
$$

where the isomorphism induces the map $g_{n}: \mathbb{W}_{n}\left(\mathcal{R}\left(\bar{R}_{\mathcal{U}}\right)\right) \rightarrow \mathbb{W}_{n}\left(\bar{R}_{\mathcal{U}} / p \bar{R}_{\mathcal{U}}\right)$.

We prove the lemma by induction on $n$. It follows from what we have just seen 2.26] and 2.17 that the map $A_{\text {cris }}^{\nabla}\left(\bar{R}_{\mathcal{U}}\right) / p A_{\text {cris }}^{\nabla}\left(\bar{R}_{\mathcal{U}}\right) \rightarrow \mathbb{A}_{\text {cris }, 1, \overline{\mathrm{K}}}^{\prime}\left(\bar{R}_{\mathcal{U}}\right)$ is injective and that its cokernel is annihilated by any element of $\mathbb{I}$.

Since $A_{\text {cris }}^{\nabla}\left(\bar{R}_{\mathcal{U}}\right)$ has no $p$-torsion by [Bri, Prop. 6.1.4] we have an exact sequence

$$
0 \longrightarrow A_{\text {cris }}^{\nabla}\left(\bar{R}_{\mathcal{U}}\right) / p^{n} A_{\text {cris }}^{\nabla}\left(\bar{R}_{\mathcal{U}}\right) \stackrel{p}{\longrightarrow} A_{\text {cris }}^{\nabla}\left(\bar{R}_{\mathcal{U}}\right) / p^{n+1} A_{\text {cris }}^{\nabla}\left(\bar{R}_{\mathcal{U}}\right) \longrightarrow A_{\text {cris }}^{\nabla}\left(\bar{R}_{\mathcal{U}}\right) / p A_{\text {cris }}^{\nabla}\left(\bar{R}_{\mathcal{U}}\right) \longrightarrow 0
$$

One checks that it is compatible with the exact sequence obtained by taking the localizations of the sequence in 2.26(b). Due to 2.26(b) the map $\mathbb{A}_{\text {cris,n+1,K}}^{\prime \nabla}\left(\bar{R}_{\mathcal{U}}\right) \rightarrow \mathbb{A}_{\text {cris, } 1, \overline{\mathrm{K}}}^{\prime \nabla}\left(\bar{R}_{\mathcal{U}}\right)$ is the map

$$
\frac{A_{\text {cris }}}{p^{n} A_{\text {cris }}} \otimes_{W_{n}}\left(\mathbb{W}_{n}\left(\bar{R}_{\mathcal{U}}\right)\right) \rightarrow \frac{A_{\text {cris }}}{p A_{\text {cris }}} \otimes_{\mathcal{O}_{\bar{K}} / p \mathcal{O}_{\bar{K}}}\left(\mathcal{O}_{\mathfrak{X}} / p \mathcal{O}_{\mathfrak{X}}\left(\bar{R}_{\mathcal{U}}\right)\right)
$$

which by construction induces the map $\mathbb{W}_{n}\left(\bar{R}_{\mathcal{U}}\right) \rightarrow\left(\mathcal{O}_{\mathfrak{X}_{\bar{K}}} / p \mathcal{O}_{\mathfrak{X}_{\bar{K}}}\right)\left(\bar{R}_{\mathcal{U}}\right)$ given by the natural projection and Frobenius to the $n-1$-th power. In particular since $n \geq 2$ this map factors via $\bar{R}_{\mathcal{U}} / p \bar{R}_{\mathcal{U}}$ by 2.17. The inductive step follows from this using the inductive hypothesis. 


\subsection{The sheaf $\mathbb{A}_{\text {cris,M }}$.}

In this section we assume that $\mathcal{O}_{K}=\mathbb{W}(k)$ is absolutely unramified. Write $\mathcal{O}_{M_{0}}$ for the ring of integers of the maximal unramified extension $M_{0}$ of $K$ in $M$. Recall that in lemma 2.13 we have described $v_{X, M}^{*}\left(\mathcal{O}_{X}\right)$ as the subsheaf $\mathcal{O}_{\mathfrak{X}_{M}}^{\text {un }}$ of $\mathcal{O}_{\mathfrak{X}_{M}}$ introduced in 2.10, It is a sheaf of $\mathcal{O}_{M_{0}}$-algebras. For every $n \geq 1$ let us define the sheaf $\mathbb{W}_{X, n, M}:=\mathbb{W}_{n}\left(\mathcal{O}_{\mathfrak{X}_{M}} / p \mathcal{O}_{\mathfrak{X}_{M}}\right) \otimes_{\mathcal{O}_{M_{0}}} \mathcal{O}_{\mathfrak{X}_{M}}^{\text {un }}$ of $\mathcal{O}_{M_{0}}$-algebras and the morphism of sheaves of $\mathcal{O}_{\mathfrak{X}_{M}}^{\text {un }}$-algebras $\theta_{X, n, M}: \mathbb{W}_{X, n, M} \longrightarrow \mathcal{O}_{\mathfrak{X}_{M}} / p^{n} \mathcal{O}_{\mathfrak{X}_{M}}$ associated to the following map of pre-sheaves. Let $(\mathcal{U}, \mathcal{W})$ be an object of $\mathfrak{X}_{M}$ such that $\mathcal{U}=\operatorname{Spf}\left(R_{\mathcal{U}}\right)$ is affine, let $\left.R_{\mathcal{U}}^{\text {un }}:=\mathcal{O}_{\mathfrak{X}_{M}}^{\text {un }}(\mathcal{U}, \mathcal{W})\right)$ and let $S=\mathcal{O}_{\mathfrak{X}_{M}}(\mathcal{U}, \mathcal{W})$. Then $S$ contains $R_{\mathcal{U}}^{\text {un }}$ and we define

$$
\theta_{n,(\mathcal{U}, \mathcal{W})}: \mathbb{W}_{n}(S / p S) \otimes_{\mathcal{O}_{M_{0}}} R_{\mathcal{U}}^{\mathrm{un}} \longrightarrow S / p^{n} S \text { by }(x \otimes r) \rightarrow c_{n}(x) r .
$$

Let $\mathcal{I}_{X, n, M}$ denote the sheaf of ideals $\operatorname{Ker}\left(\theta_{X, n, M}\right)$. Due to [B1, Thm. I.2.4.1] one knows that the $\mathbb{W}(k)$-DP envelope $\mathbb{A}_{\text {cris,n,M }}$ of $\mathbb{W}_{X, n, M}$ with respect to $\mathcal{I}_{X, n, M}$ exists. The main point of this section is an explicit description of $\mathbb{A}_{\text {cris,n,M }}$ in theorem 2.31 which will be used in the sequel.

Let $\mathcal{U}=\operatorname{Spf}\left(R_{\mathcal{U}}\right)$ denote a small affine open as in 2.1 with parameters $T_{1}, T_{2}, \ldots, T_{d} \in R_{\mathcal{U}}^{\times}$. For every $n \geq 0$ define $R_{\mathcal{U}, n}:=R_{\mathcal{U}}\left[\zeta_{n}, T_{1}^{1 / p^{n}}, \ldots, T_{d}^{1 / p^{n}}\right]$, where $R_{\mathcal{U}, 0}=R_{\mathcal{U}}$, $\zeta_{n}$ is a primitive $p^{n}$-th root of 1 such that $\zeta_{n+1}^{p}=\zeta_{n}$ and $T_{i}^{1 / p^{n}}$ is a fixed $p^{n}$-th root of $T_{i}$ in $\bar{R}_{\mathcal{U}}$ such that $\left(T_{i}^{1 / p^{n+1}}\right)^{p}=T_{i}^{1 / p^{n}}$. We consider the category $\mathfrak{U}_{n, M}$ consisting of objects $(\mathcal{V}, \mathcal{W})$ and a morphism to $\left(\mathcal{U}, \operatorname{Spf}\left(R_{\mathcal{U}, n}\right) \otimes_{\mathcal{O}_{K}} M\right)$. The morphisms are the morphisms as objects over $\left(\mathcal{U}, \operatorname{Spf}\left(R_{\mathcal{U}, n}\right) \otimes_{\mathcal{O}_{K}} M\right)$. The covering families of an object $(\mathcal{V}, \mathcal{W})$ are the covering families as an object of $\mathfrak{X}_{M}$. There is a morphism of sites $\iota: \mathfrak{X}_{M} \longrightarrow \mathfrak{U}_{n, M}$ sending $(\mathcal{V}, W)$ to $(\mathcal{V}, W) \times\left(X, X_{K}\right)$ $\left(\mathcal{U}, \operatorname{Spf}\left(R_{\mathcal{U}, n}\right) \otimes_{\mathcal{O}_{K}} M\right)$. Given a sheaf on $\mathfrak{X}_{M}$ we write $\left.\mathcal{F}\right|_{\mathfrak{U}_{n, M}}$ for $\iota^{*}(\mathcal{F})$.

Let $(\mathcal{V}, \mathcal{W}) \in \mathfrak{U}_{n, M}$ with $\mathcal{V}=\operatorname{Spf}\left(R_{\mathcal{V}}\right)$ affine and put $S:=\mathcal{O}_{\mathfrak{X}_{M}}(\mathcal{V}, \mathcal{W})$. Note that $T_{i}^{1 / p^{n}} \in$ $R_{\mathcal{U}, n} \subset S$ for all $1 \leq i \leq d$. Denote by

$$
\widetilde{T}_{i}:=\left(\left[T_{i}\right],\left[T_{i}^{1 / p}\right], \cdots,\left[T_{i}^{1 / p^{n}}\right], \cdots\right) \in \lim _{\infty \leftarrow n} \mathbb{W}_{n}\left(R_{\mathcal{U}, n} / p R_{\mathcal{U}, n}\right),
$$

where the inverse limit is taken with respect to $\mathbb{W}_{n+1}\left(R_{\mathcal{U}, n+1} / p R_{\mathcal{U}, n+1}\right) \rightarrow \mathbb{W}_{n}\left(R_{\mathcal{U}, n} / p R_{\mathcal{U}, n}\right)$ the natural projection $\mathbb{W}_{n+1}\left(R_{\mathcal{U}, n+1} / p R_{\mathcal{U}, n+1}\right) \rightarrow \mathbb{W}_{n}\left(R_{\mathcal{U}, n+1} / p R_{\mathcal{U}, n+1}\right)$ and the map induced by Frobenius $R_{\mathcal{U}, n+1} / p R_{\mathcal{U}, n+1} \rightarrow R_{\mathcal{U}, n} / p R_{\mathcal{U}, n}$. The image of $\widetilde{T}_{i}$ in $\mathbb{W}_{n}\left(R_{\mathcal{U}, n} / p R_{\mathcal{U}, n}\right)$ is the Teichmüller $\operatorname{lift}\left(T_{i}^{1 / p^{n}}, 0, \ldots, 0\right)$ of $T_{i}^{1 / p^{n}}$. Write

$$
X_{i}:=1 \otimes T_{i}-\widetilde{T}_{i} \otimes 1 \in \mathbb{W}_{n}\left(R_{\mathcal{U}, n} / p R_{\mathcal{U}, n}\right) \otimes_{\mathcal{O}_{K}} R_{\mathcal{U}}
$$

for $i=1, \ldots, d$. They are naturally elements of $\mathbb{W}_{n}(S / p S) \otimes_{\mathcal{O}_{K}} R_{\mathcal{V}}$.

Lemma 2.30. The kernel of the map $\theta_{n,(\mathcal{V}, \mathcal{W})}: \mathbb{W}_{n}(S / p S) \otimes_{\mathcal{O}_{M_{0}}} R_{\mathcal{V}}^{\mathrm{un}} \rightarrow S / p^{n} S$ is the ideal generated by $\left(\xi_{n}, X_{1}, \ldots, X_{d}\right)$.

Proof. The kernel of the ring homomorphism $R_{0} / p^{n} R_{0} \otimes R_{0} / p^{n} R_{0} \rightarrow R_{0} / p^{n} R_{0}$ defined by $x \otimes y \rightarrow$ $x y$ is the ideal $I=\left(T_{1} \otimes 1-1 \otimes T_{1}, \ldots, T_{d} \otimes 1-1 \otimes T_{d}\right)$. Since $R_{0} / p^{n} R_{0} \rightarrow R_{\mathcal{V}} / p^{n} R_{\mathcal{V}} \rightarrow R_{\mathcal{V}}^{\text {un }} / p^{n} R_{\mathcal{V}}^{\text {un }}$ are étale morphisms, then $I$ generates the kernel of $R_{\mathcal{V}}^{\text {un }} / p^{n} R_{\mathcal{V}}^{\text {un }} \otimes R_{\mathcal{V}}^{\text {un }} / p^{n} R_{\mathcal{V}}^{\text {un }} \rightarrow R_{\mathcal{V}}^{\text {un }} / p^{n} R_{\mathcal{V}}^{\text {un }}$. Base changing via $R_{\mathcal{V}}^{\text {un }} / p^{n} R_{\mathcal{V}}^{\text {un }} \rightarrow S / p^{n} S$ we conclude that $I$ generates the kernel of $S / p^{n} S \otimes$ $R_{\mathcal{V}}^{\text {un }} / p^{n} R_{\mathcal{V}}^{\text {un }} \rightarrow S / p^{n} S$. By lemma 2.19 the kernel of $c_{n}: \mathbb{W}_{n}(S / p S) \rightarrow S / p^{n} S$ is generated by $\xi_{n}$. The conclusion follows. 
For every $(\mathcal{V}, \mathcal{W}) \in \mathfrak{U}_{n, M}$ let $\mathbb{A}_{\text {cris,n,M }}^{\nabla}(\mathcal{V}, \mathcal{W})\left\langle X_{1}, \ldots, X_{d}\right\rangle$ be the DP envelope of the polynomial algebra $\mathbb{A}_{\text {cris, }, \mathrm{n}, \mathrm{n}}(\mathcal{V}, \mathcal{W})\left[X_{1}, \ldots, X_{d}\right]$ with respect to the ideal $\left(X_{1}, \ldots, X_{d}\right)$. As explained in [Bri, §6], we have

$$
\mathbb{A}_{\text {cris,n,M }}^{\nabla}(\mathcal{V}, \mathcal{W})\left\langle X_{1}, \ldots, X_{d}\right\rangle=\mathbb{A}_{\text {cris, }, \mathrm{M}, \mathrm{M}}^{\nabla}(\mathcal{V}, \mathcal{W})\left[X_{i, 0}, X_{i, 1}, \ldots\right]_{1 \leq i \leq d} /\left(p X_{i, m+1}-X_{i, m}^{p}\right)_{1 \leq i \leq d, m \geq 0}
$$

where $X_{i, j}=\gamma^{j+1}\left(X_{i}\right)$ and $\gamma: z \mapsto(p-1) ! z^{[p]}$. In particular it is a free $\mathbb{A}_{\text {cris,n,M }}^{\nabla}(\mathcal{V}, \mathcal{W})$-module with bases given by the monomials in the variables $X_{i, 0}, X_{i, 1}, \ldots$ for $1 \leq i \leq d$ in which each variable $X_{i, j}$ appears with degree $\leq p-1$. We conclude that $(\mathcal{V}, \mathcal{W}) \rightarrow \mathbb{A}_{\text {cris,n,M }}^{\nabla}(\mathcal{V}, \mathcal{W})\left\langle X_{1}, \ldots, X_{d}\right\rangle$ is a sheaf and that the ideal generated by $\operatorname{Ker}\left(\theta_{n, M}\right)\left(\subset \mathbb{A}_{\text {cris,n,M }}^{\nabla}\right)$ and $\left(X_{1, j}, \cdots, X_{d, j}\right)_{j \in \mathbb{N}}$ has $\mathbb{W}(k)-$ DP structure. Write $\mathbb{A}_{\text {cris }, \mathfrak{U}_{n, M}}$ for the sheaf $\mathbb{A}_{\text {cris,n,M}}^{\nabla} \mid \mathfrak{U}_{n, M}\left\langle X_{1}, \ldots, X_{d}\right\rangle$. Let $\theta_{\mathfrak{U}_{n, M}}: \mathbb{A}_{\text {cris, } \mathfrak{U}_{\mathrm{n}, \mathrm{M}}} \longrightarrow$ $\mathcal{O}_{\mathfrak{X}_{M}} /\left.p^{n} \mathcal{O}_{\mathfrak{X}_{M}}\right|_{\mathfrak{U}_{n, M}}$ be the map sending $X_{i, j} \mapsto 0$ and coinciding with $\left.\theta_{n, M}\right|_{\mathfrak{U}_{n, M}}$ on $\left.\mathbb{A}_{\text {cris,n,M }}\right|_{\mathfrak{U}_{n, M}}$. For every $(\mathcal{V}, \mathcal{W}) \in \mathfrak{U}_{n, M}$ with $\mathcal{V}:=\operatorname{Spf}\left(R_{\mathcal{V}}\right)$ affine, define the map

$$
R_{0}=\mathbb{W}(k)\left\{T_{1}^{ \pm 1}, \ldots, T_{d}^{ \pm 1}\right\} \longrightarrow \mathbb{A}_{\text {cris }, \mathrm{n}, \mathrm{M}}^{\nabla}(\mathcal{V}, \mathcal{W})\left\langle X_{1}, \ldots, X_{d}\right\rangle
$$

of $\mathbb{W}(k)$-algebras setting $T_{i} \rightarrow \widetilde{T}_{i} \otimes 1+1 \otimes X_{i}$ for every $1 \leq i \leq d$. As $T_{i}$ is a unit in $R_{\mathcal{U}}$, then $\widetilde{T}_{i}$ is a unit in $\mathbb{W}_{n}(S / p S)$ where $S:=\mathcal{O}_{\mathfrak{x}_{M}}(\mathcal{V}, \mathcal{W})$. Since $X_{i}^{p}=p X_{i}^{[p]}$ is nilpotent in $\mathbb{A}_{\text {cris,n,M }}^{\nabla}(\mathcal{V}, \mathcal{W})\left\langle X_{1}, \ldots, X_{d}\right\rangle$, also $\widetilde{T}_{i} \otimes 1+1 \otimes X_{i}$ is a unit and hence the displayed ring homomorphism is well defined. Let us now look at the following diagram of $\mathbb{W}(k)$-algebras

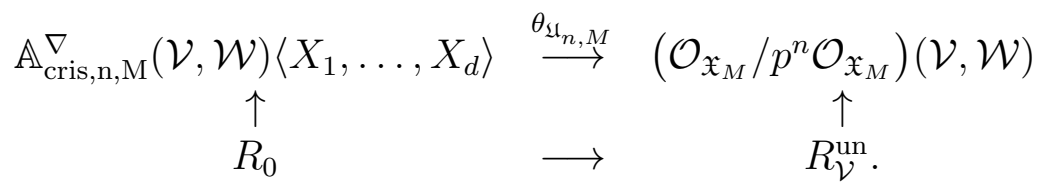

The diagram is commutative since $\theta_{\mathfrak{U}_{n, M}}\left(\widetilde{T}_{i} \otimes 1+1 \otimes X_{i}\right)=\theta_{\mathfrak{U}_{n, M}}\left(\widetilde{T}_{i}\right)=\left(T_{i}^{1 / p^{n}}\right)^{p^{n}}=T_{i}$. The kernel of $\theta_{\mathfrak{U}_{n, M}}(\mathcal{V}, \mathcal{W})$ is the DP ideal generated by $\operatorname{Ker}\left(\theta_{n, M}\right)(\mathcal{U}, \mathcal{W})$ and by $\left(X_{1, j}, \ldots, X_{d, j}\right)_{j \in \mathbb{N}}$

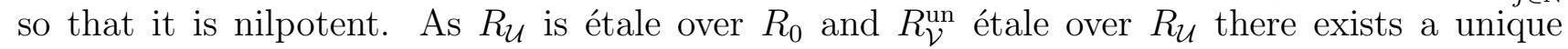
homomorphism

$$
R_{\mathcal{V}}^{\mathrm{un}} \longrightarrow \mathbb{A}_{\text {cris,n,M }}^{\nabla}(\mathcal{V}, \mathcal{W})\left\langle X_{1}, \ldots, X_{d}\right\rangle
$$

of $R_{0}$-algebras making both triangles in the above diagram commutative. We thus get a map $\mathbb{W}_{n, M}(\mathcal{V}, \mathcal{W}) \otimes_{\mathcal{O}_{M_{0}}} \mathcal{O}_{\mathfrak{X}_{M}}^{\text {un }}(\mathcal{V}, \mathcal{W}) \rightarrow \mathbb{A}_{\text {cris,n,M }}^{\nabla}(\mathcal{V}, \mathcal{W})\left\langle X_{1}, \ldots, X_{d}\right\rangle$. Passing to the associated sheaves we get a map of sheaves

$$
h_{\mathfrak{U}_{n, M}}:\left.\mathbb{W}_{X, n, M}\right|_{\mathfrak{U}_{n, M}} \rightarrow \mathbb{A}_{\text {cris }, \mathfrak{U}_{\mathrm{n}, \mathrm{M}}} .
$$

It follows from 2.30 that the image of $\left.\mathcal{I}_{X, n, M}\right|_{\mathfrak{U}_{n, M}}$ is contained in the ideal generated by $\operatorname{Ker}\left(\theta_{n, M}\right)$ and $\left(X_{1, j}, \cdots, X_{d, j}\right)_{j \in \mathbb{N}}$ which has $\mathbb{W}(k)$-DP structure.

Theorem 2.31. (1) The $\mathbb{W}(k)-D P$ envelope $\mathbb{A}_{\text {cris,n,M }}$ of $\mathbb{W}_{X, n, M}$ with respect to $\mathcal{I}_{X, n, M}$ exists.

(2) For every small affine $\mathcal{U}$ of $X^{\text {et }}$ the sheaf $\mathbb{A}_{\text {cris }, \mathfrak{U}_{n, M}}$ with the ideal $\operatorname{Ker}\left(\theta_{\mathfrak{U}_{n, M}}\right)$ endowed with its $\mathbb{W}(k)-D P$ structure, is the $\mathbb{W}(k)-D P$ envelope of $\left.\mathbb{W}_{X, n, M}\right|_{\mathfrak{U}_{n, M}}$ with respect to $\left.\mathcal{I}_{X, n, M}\right|_{\mathfrak{U}_{n, M}}$. In particular the restriction of $\mathbb{A}_{\text {cris,n,M }}$ to $\mathfrak{U}_{n, M}$ is $\mathbb{A}_{\text {cris, } \mathfrak{U}_{\mathrm{n}, \mathrm{M}}}$.

(3) Let $M_{1} \subset M_{2}$ be a Galois extension. The morphism $\beta_{M_{1}, M_{2}}^{*}\left(\mathbb{W}_{X, n, M_{1}} \otimes_{\mathcal{O}_{M_{1}}^{\text {un }}} \mathcal{O}_{\mathfrak{X}_{M_{1}}}^{\text {un }}\right) \longrightarrow$ $\mathbb{W}_{X, n, M_{2}} \otimes_{\mathcal{O}_{M_{2}}^{\text {un }}} \mathcal{O}_{\mathfrak{X}_{M_{2}}}^{\text {un }}$ induces an isomorphism $\beta_{M_{1}, M_{2}}^{*}\left(\mathbb{A}_{\text {cris,n, } \mathrm{M}_{1}}\right) \cong \mathbb{A}_{\text {cris,n, } \mathrm{M}_{2}}$ of $\mathbb{W}(k)-D P$ sheaves of algebras. 
Proof. (1) The existence is a formal consequence of [B1, Thm. I.2.4.1].

(2) Consider a $\mathbb{W}(k)$-DP sheaf of algebras $\mathcal{G}$ and a morphism $f:\left.\mathbb{W}_{X, n, M}\right|_{\mathfrak{U}_{n, M}} \rightarrow \mathcal{G}$ sending $\left.\mathcal{I}_{X, n, M}\right|_{\mathfrak{U}_{n, M}}$ to the DP ideal of $\mathcal{G}$. Due to 2.24 and 2.23 the induced map $\left.\mathbb{W}_{n, M}\right|_{\mathfrak{U}_{n, M}} \rightarrow \mathcal{G}$

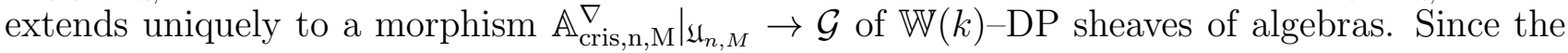
section $X_{i}=1 \otimes T_{i}-\widetilde{T}_{i} \otimes 1$ of $\mathbb{W}_{X, n, M}$ lies in $\operatorname{Ker}\left(\theta_{\mathfrak{U}_{n, M}}\right)$ we can uniquely extend such map to a morphism $\mathbb{A}_{\text {cris,n,M }}^{\nabla}{\mid \mathfrak{U}_{n, M}}^{\nabla}\left\langle X_{1}, \ldots, X_{d}\right\rangle \rightarrow \mathcal{G}$. This proves the first part of the claim. The second part follows from the first and lemma 2.23 .

(3) Via the morphism $\beta_{M_{1}, M_{2}}^{*}\left(\mathbb{W}_{n, M_{1}} \otimes_{\mathcal{O}_{M_{1}}^{\text {un }}} \mathcal{O}_{\mathfrak{X}_{M_{1}}}^{\text {un }}\right) \longrightarrow \mathbb{W}_{n, M_{2}} \otimes_{\mathcal{O}_{M_{2}}^{\text {un }}} \mathcal{O}_{\mathfrak{X}_{M_{2}}}^{\text {un }}$ one checks that $\beta_{M_{1}, M_{2}}^{*}\left(\theta_{X, n, M_{1}}\right)$ and $\theta_{X, n, M_{2}}$ are compatible so that $\beta_{M_{1}, M_{2}}^{*}\left(\mathcal{I}_{X, n, M_{1}}\right)$ is sent to $\mathcal{I}_{X, n, M_{2}}$. Due to the

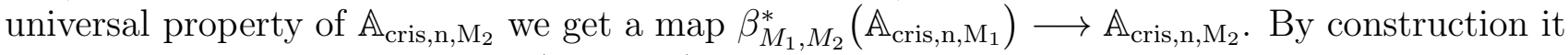

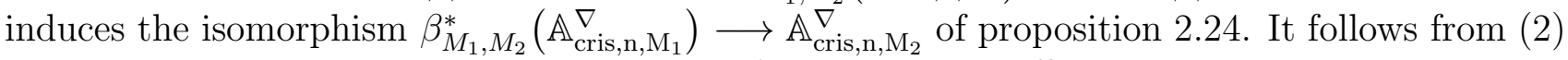
that the restriction to $\mathfrak{U}_{n, M_{1}}$ sends $X_{i} \mapsto X_{i}$ for every small affine $\mathcal{U}$ and in particular it is an isomorphism. This implies the claim.

Let $\mathcal{U}=\operatorname{Spf}\left(R_{\mathcal{U}}\right)$ denote a small affine open in $X^{\text {et }}$. Choose $T_{1}, \ldots T_{d} \in R_{\mathcal{U}}^{\times}$parameters and let $F_{\mathcal{U}}: R_{\mathcal{U}} \rightarrow R_{\mathcal{U}}$ be the unique map inducing Frobenius on $\mathcal{O}_{K}$ and sending $T_{i} \mapsto T_{i}^{p}$. Denote by $F_{\mathcal{U}}: \mathcal{O}_{\mathcal{U}} \rightarrow \mathcal{O}_{\mathcal{U}}$ the induced map of sheaves on $\mathcal{U}^{\text {et }}$. Taking $v_{\mathcal{U}, M}^{*}$ it provides a morphism $F_{\mathcal{U}}$ on $\left.\mathcal{O}_{\mathfrak{X}_{M}}^{\text {un }}\right|_{\mathfrak{U}_{M}}$. Let

$$
\varphi_{\mathfrak{U}_{M}, n}:\left.\left.\mathbb{W}_{X, n, M}\right|_{\mathfrak{U}_{M}} \cong \mathbb{W}_{\mathcal{U}, M, n} \longrightarrow \mathbb{W}_{\mathcal{U}, M, n} \cong \mathbb{W}_{X, n, M}\right|_{\mathfrak{U}_{M}}
$$

be the map of sheaves associated to the map of pre-sheaves $\varphi_{\mathcal{U}, n} \otimes F_{\mathcal{U}}: \mathbb{W}_{n, \mathcal{U}, M} \otimes_{\mathcal{O}_{M_{0}}} \mathcal{O}_{\mathfrak{U}_{M}}^{\text {un }} \rightarrow$ $\mathbb{W}_{n, \mathcal{U}, M} \otimes_{\mathcal{O}_{M_{0}}} \mathcal{O}_{\mathfrak{U}_{M}}^{\text {un }}$.

Corollary 2.32. 1) The morphism $\varphi_{\mathfrak{U}_{M}, n}$ on $\left.\mathbb{W}_{X, n, M}\right|_{\mathfrak{U}_{M}}$ extends uniquely to an operator $\varphi_{\mathfrak{U}_{M}, n}$ on $\left.\mathbb{A}_{\text {cris,n,M }}\right|_{\mathfrak{U}_{M}}$, called Frobenius, compatible with Frobenius on $\mathbb{A}_{\text {cris,n,M }}^{\nabla} \mid \mathfrak{U}_{M}$ defined in proposition 2.24.

2) Via the identification given in theorem 2.31 the restriction $\varphi_{\mathfrak{U}_{n, M}}$ of $\varphi_{\mathfrak{U}_{M}, n}$ to $\mathfrak{U}_{n, M}$ is uniquely determined by requiring that it induces Frobenius on $\mathbb{A}_{\text {cris,n,M }}^{\nabla} \mid \mathfrak{U}_{n, M}$ and sends $X_{i} \mapsto$ $1 \otimes T_{i}^{p}-\widetilde{T}_{i}^{p} \otimes 1=X_{i}\left(\sum_{h=1}^{p-1} T_{i}^{h} \widetilde{T}_{i}^{p-h}\right)$ for $i=1, \ldots, d$.

3) The isomorphism $\left.\beta_{M_{1}, M_{2}}^{*}\left(\left.\mathbb{A}_{\text {cris,n, }, \mathrm{M}_{1}}\right|_{\mathfrak{U}_{M_{1}}}\right) \cong \mathbb{A}_{\text {cris,n, }, \mathrm{M}_{2}}\right|_{\mathfrak{U}_{M_{2}}}$ of $\mathbb{W}(k)$-DP sheaves of algebras is compatible with Frobenius.

Proof. The fact that Frobenius on $\left.\mathbb{W}_{X, n, M}\right|_{\mathfrak{U}_{M}}$ extends to $\mathbb{A}_{\text {cris,n,M }}^{\nabla} \mid \mathfrak{U}_{M}$ follows from proposition 2.24 .

(2) For $i=1, \ldots, d$ we compute that $\varphi_{\mathfrak{U}_{n, M}}\left(X_{i}\right)=1 \otimes T_{i}^{p}-\widetilde{T}_{i}^{p} \otimes 1=X_{i}\left(\sum_{h=1}^{p-1} T_{i}^{h} \widetilde{T}_{i}^{p-h}\right)$ so that $\varphi_{\mathfrak{U}_{n, M}}\left(X_{i}\right)$ admits divided powers. This implies that $\varphi_{\mathfrak{U}_{n, M}}\left(\mathcal{I}_{\mathcal{U}, n, M}\right)$ admits divided powers so that by the universal property of $\left.\mathbb{A}_{\text {cris,n,M }}\right|_{\mathfrak{U}_{n, M}}$ (see 2.31) the morphism $\varphi_{\mathfrak{U}_{n, M}}$ extends to $\left.\mathbb{A}_{\text {cris,n,M }}\right|_{\mathfrak{U}_{n, M},}$.

(1) Since $\varphi_{\mathfrak{U}_{n, M}}\left(\mathcal{I}_{\mathcal{U}, n, M}\right)$ admits divided powers in $\left.\mathbb{A}_{\text {cris,n,M }}\right|_{\mathfrak{U}_{n, M}}$ by $(2)$ then also $\varphi_{\mathfrak{U}_{M}, n}\left(\mathcal{I}_{\mathcal{U}, n, M}\right)$ admits divided powers in $\left.\mathbb{A}_{\text {cris,n,M }, M}\right|_{\mathfrak{U}_{M}}$. By the universal property of $\left.\mathbb{A}_{\text {cris,n,M }}\right|_{\mathfrak{U}_{M}}$, which follows from 2.31 and 2.23 , the morphism $\varphi_{\mathfrak{U}_{n, M}}$ extends to $\left.\mathbb{A}_{\text {cris,n,M }}\right|_{\mathfrak{U}_{M}, n}$.

(3) It suffices to prove the claim after restricting to $\mathfrak{U}_{n, M_{2}}$. In this case it follows from (1) and theorem 2.31 . 
Let $r_{X, n+1, M}: \mathbb{W}_{n+1, M} \otimes_{\mathcal{O}_{M_{0}}} \mathcal{O}_{\mathfrak{X}_{M}}^{\text {un }} \rightarrow \mathbb{W}_{n, M} \otimes_{\mathcal{O}_{M_{0}}} \mathcal{O}_{\mathfrak{X}_{M}}^{\text {un }}$ be the morphism which is the identity on $\mathcal{O}_{\mathfrak{X}_{M}}^{\text {un }}$ and is reduction composed with Frobenius on $\mathbb{W}_{n+1, M} \rightarrow \mathbb{W}_{n, M}$. Then we have an inclusion $r_{X, n+1, M}\left(\operatorname{Ker}\left(\theta_{X, n+1}\right)\right) \subset \operatorname{Ker}\left(\theta_{X, n}\right)$. Hence $r_{X, n+1, M}$ defines a map $\mathbb{A}_{\text {cris,n+1,M }} \rightarrow \mathbb{A}_{\text {cris,n, }, \mathrm{M}}$. Let $\mathbb{A}_{\text {cris,M }}$ denote the sheaf in $\operatorname{Sh}\left(\mathfrak{X}_{M}\right)^{\mathbb{N}}$ defined by the family $\left\{\mathbb{A}_{\text {cris,n,M }}\right\}_{n}$ with the transition functions $r_{X, n, M}$. It is the $\mathbb{W}(k)$-DP envelope of $\left\{\mathbb{W}_{n, M} \otimes_{\mathcal{O}_{M_{0}}} \mathcal{O}_{\mathfrak{X}_{M}}^{\text {un }}\right\}_{n}$ with respect to the ideals $\left\{\operatorname{Ker}\left(\theta_{X, n}\right)\right\}_{n}$. For every small affine $\mathcal{U}$ and parameters $T_{1}, \ldots, T_{d}$ denote by $\varphi_{\mathcal{U}}: \mathbb{A}_{\text {cris }, \mathrm{M}} \rightarrow \mathbb{A}_{\text {cris }, \mathrm{M}}$ the map in $\operatorname{Sh}\left(\mathfrak{U}_{M}^{\bullet}\right)^{\mathbb{N}}$ defined by $\left\{\varphi_{\mathcal{U}, n}\right\}_{n}$.

Lemma 2.33. For every $m^{\prime}>m>n$ the maps $r_{X, m^{\prime}, M} \circ \ldots \circ r_{X, m+1, M}: \mathbb{A}_{\text {cris }, \mathrm{m}^{\prime}, \mathrm{M}} \rightarrow \mathbb{A}_{\text {cris }, \mathrm{m}, \mathrm{M}}$ induce an isomorphism $\mathbb{A}_{\text {cris }, \mathrm{m}^{\prime}, \mathrm{M}} / p^{n} \mathbb{A}_{\text {cris, } \mathrm{m}^{\prime}, \mathrm{M}} \longrightarrow \mathbb{A}_{\text {cris }, \mathrm{m}, \mathrm{M}} / p^{n} \mathbb{A}_{\text {cris }, \mathrm{m}, \mathrm{M}}$.

With the notations of [2.31, for every small $\mathcal{U}$ of $X^{\mathrm{et}}$ the restriction of this sheaf to $\mathfrak{U}_{n, M}$ is isomorphic to $\left.\mathbb{A}_{\text {cris,n,M }}^{\prime}\right|_{\mathfrak{U}_{M, n}}\left\langle X_{1}, \ldots, X_{d}\right\rangle$.

Proof. It suffices to prove the two claims restricting to $\mathfrak{U}_{n, M}$ for every small object $\mathcal{U}$. They follow from theorem 2.31 and lemma 2.27.

Denote by $\mathbb{A}_{\text {cris,n,M }}^{\prime}$ the sheaf $\mathbb{A}_{\text {cris, }, \mathrm{M}} / p^{n} \mathbb{A}_{\text {cris,m,M }}$ for $m>n$ introduced in 2.33, It is the $\mathbb{W}(k)$-DP envelope of $\mathbb{W}_{X, n, M}$ with respect to the kernel of the map $\theta_{X, n}^{\prime}: \mathbb{W}_{n, M} \otimes_{\mathcal{O}_{M_{0}}} \mathcal{O}_{\mathfrak{X}_{M}}^{\text {un }} \rightarrow$ $\mathcal{O}_{\mathfrak{X}_{M}} / p^{n} \mathcal{O}_{\mathfrak{X}_{M}}$ induced by $\theta_{n, M}^{\prime}$ on $\mathbb{W}_{n, M}$ and the natural projection $\mathcal{O}_{\mathfrak{X}_{M}}^{\text {un }} \rightarrow \mathcal{O}_{\mathfrak{X}_{M}} / p^{n} \mathcal{O}_{\mathfrak{X}_{M}}$. For every small affine $\mathcal{U}$ and parameters $T_{1}, \ldots, T_{d}$ denote by $\varphi_{\mathcal{U}, n}^{\prime}: \mathbb{A}_{\text {cris }, \mathcal{U}, \mathrm{n}, \mathrm{M}}^{\prime} \rightarrow \mathbb{A}_{\text {cris }, \mathcal{U}, \mathrm{n}, \mathrm{M}}$ the map defined by $\varphi_{n} \otimes F_{\mathcal{U}}$ on $\left.\mathbb{W}_{n, M}\right|_{\mathfrak{U}_{n, M}} \otimes_{\mathcal{O}_{M_{0}}} \mathcal{O}_{\mathfrak{X}_{M}}^{\text {un }}$. Let $\mathbb{A}_{\text {cris,M }}^{\prime}:=\left\{\mathbb{A}_{\text {cris,n,M }}^{\prime}\right\}_{n}$ be the associated system of sheaves, where the transition maps $r_{X, n+1, M}^{\prime}: \mathbb{A}_{\text {cris,n+1,M }}^{\prime} \rightarrow \mathbb{A}_{\text {cris,n,M }}^{\prime}$ a induced by the transition maps $\left\{r_{X, m, M}\right\}_{m}$. By construction we have a natural morphism $q_{X, n}: \mathbb{A}_{\text {cris,n,M }}^{\prime} \rightarrow \mathbb{A}_{\text {cris,n,M }}$ for every $n \in \mathbb{N}$ and hence a map $q_{X}:=\left\{q_{X, n}\right\}_{n}: \mathbb{A}_{\text {cris,M }}^{\prime} \rightarrow \mathbb{A}_{\text {cris, } \mathrm{M}}$. For every small affine $\mathcal{U}$ and parameters $T_{1}, \ldots, T_{d}$ denote by $\varphi_{\mathcal{U}}^{\prime}:=\left\{\varphi_{\mathcal{U}, n}^{\prime}\right\}_{n}$.

Following [Bri] define $A_{\text {cris, }}\left(\bar{R}_{\mathcal{U}}\right)$ as the $p$-adic completion of the $\mathbb{W}(k)-\mathrm{DP}$ envelope of $\mathbb{W}\left(\mathcal{R}\left(\bar{R}_{\mathcal{U}}\right)\right) \otimes_{\mathcal{O}_{K}} R_{\mathcal{U}}$ with respect to the kernel of the map $\mathbb{W}\left(\mathcal{R}\left(\bar{R}_{\mathcal{U}}\right)\right) \otimes_{\mathcal{O}_{K}} R_{\mathcal{U}} \rightarrow \widehat{\bar{R}}_{\mathcal{U}}$ given by $x \otimes y \mapsto \vartheta(x) y$. Furthermore, it is proved that the operator $\varphi \otimes F_{\mathcal{U}}$ on $\mathbb{W}\left(\mathcal{R}\left(\bar{R}_{\mathcal{U}}\right)\right) \otimes_{\mathcal{O}_{K}} R_{\mathcal{U}}$ defines an operator $\varphi$ on $A_{\text {cris,M }}\left(\bar{R}_{\mathcal{U}}\right)$. It is shown in [Bri, Prop. 6.1.8] that $A_{\text {cris, } \mathrm{M}}\left(\bar{R}_{\mathcal{U}}\right)$ is the $p$-adic completion of the algebra $A_{\text {cris }}^{\nabla}\left(\bar{R}_{\mathcal{U}}\right)\left\langle X_{1}, \ldots, X_{d}\right\rangle$. Hence

$$
A_{\text {cris }}\left(\bar{R}_{\mathcal{U}}\right) / p^{n} A_{\text {cris }}\left(\bar{R}_{\mathcal{U}}\right) \cong\left(A_{\text {cris }}^{\nabla}\left(\bar{R}_{\mathcal{U}}\right) / p^{n} A_{\text {cris }}^{\nabla}\left(\bar{R}_{\mathcal{U}}\right)\right)\left\langle X_{1}, \ldots, X_{d}\right\rangle .
$$

This and 2.33 provide a natural map $g_{\mathcal{U}, n}: A_{\text {cris }}\left(\bar{R}_{\mathcal{U}}\right) / p^{n} A_{\text {cris }}\left(\bar{R}_{\mathcal{U}}\right) \rightarrow \mathbb{A}_{\text {cris,n,M }}^{\prime}\left(\bar{R}_{\mathcal{U}}\right)$ and hence a map

$$
g_{\mathcal{U}}:=\lim _{n} g_{\mathcal{U}, n}: A_{\text {cris }}\left(\bar{R}_{\mathcal{U}}\right) \longrightarrow \mathbb{A}_{\text {cris }, \mathrm{M}}^{\prime}\left(\bar{R}_{\mathcal{U}}\right) .
$$

Proposition 2.34. 1) The map $\mathbb{A}_{\text {cris, } \mathrm{M}}^{\prime}\left(\bar{R}_{\mathcal{U}}\right) \rightarrow \mathbb{A}_{\text {cris, } \mathrm{M}}\left(\bar{R}_{\mathcal{U}}\right)$ induced by $q_{X}$ is an isomorphism.

2) The map $g_{\mathcal{U}}$ is an isomorphism and commutes with the two Frobenii.

Proof. The first claim follows from 2.33 and 2.28 . The second statement follows from the next lemma.

Lemma 2.35. For every $n \in \mathbb{N}$ the map $g_{\mathcal{U}, n}$ is injective, its cokernel is annihilated by any element of $\mathbb{I}$, it commutes with Frobenii and the transition map $\mathbb{A}_{\text {cris,n }+1, \mathrm{M}}^{\prime}\left(\bar{R}_{\mathcal{U}}\right) \rightarrow \mathbb{A}_{\text {cris,n,M }}^{\prime}\left(\bar{R}_{\mathcal{U}}\right)$ factors via $A_{\text {cris }}\left(\bar{R}_{\mathcal{U}}\right) / p^{n} A_{\text {cris }}\left(\bar{R}_{\mathcal{U}}\right)$.

Proof. It follows from lemma 2.33 and lemma 2.29. 


\subsection{Further properties of $\mathbb{A}_{\text {cris, } \mathrm{M}}^{\nabla}$ and $\mathbb{A}_{\text {cris, }, \mathrm{M}}$.}

Let us recall that we write $\mathbb{A}_{\text {cris, },}^{\nabla}$ both for the system of sheaves $\left\{\mathbb{A}_{\text {cris,n,M }}^{\nabla}\right\}_{n}$ and for $\left\{\mathbb{A}_{\text {cris, }, \mathrm{M}, \mathrm{n}}^{\prime}\right\}_{n}$. Similarly, we write $\mathbb{A}_{\text {cris, } \mathrm{M}}$ both for $\left\{\mathbb{A}_{\text {cris,n,M }}\right\}_{n}$ and for $\left\{\mathbb{A}_{\text {cris, }, \mathrm{M}}^{\prime}\right\}_{n}$. We specify which system is used when needed. Whenever $\mathbb{A}_{\text {cris, } M}$ appears we implicitly assume, as in the previous section, that $\mathcal{O}_{K}=\mathbb{W}(k)$. Consider the filtration $\left\{\operatorname{Fil}^{r}\left(\mathbb{A}_{\text {cris, } \mathrm{M}}^{\nabla}\right)\right\}_{r \in \mathbb{N}}$ defined by $\left\{\operatorname{Ker}\left(\theta_{n, M}\right)^{[r]}\right\}_{n}$ (resp. $\left.\left\{\operatorname{Ker}\left(\theta_{n, M}^{\prime}\right)^{[r]}\right\}_{n}\right)$. Analogously define the filtration $\left\{\operatorname{Fil}^{r}\left(\mathbb{A}_{\text {cris, }, \mathrm{M}}\right)\right\}_{r \in \mathbb{N}}$ given by the subsheaves $\left\{\operatorname{Ker}\left(\theta_{X, M, n}\right)^{[r]}\right\}_{n}\left(\operatorname{resp} .\left\{\operatorname{Ker}\left(\theta_{X, M, n}^{\prime}\right)^{[r]}\right\}_{n}\right)$.

Let $\mathcal{U}$ be a small affine of $X^{\text {et }}$ and choose parameters $T_{1}, \ldots, T_{d}$ in $R_{\mathcal{U}}^{\times}$. Then $\left.\mathbb{A}_{\text {cris,M }}\right|_{\mathfrak{L}_{n, M}}=$ $\mathbb{A}_{\text {cris }, \mathrm{M}}^{\nabla} \mid \mathfrak{U}_{n, M}\left\{\left\langle X_{1}, \ldots, X_{d}\right\rangle\right\}$ by 2.31 and $\left.\mathrm{Fil}^{r}\left(\mathbb{A}_{\text {cris }, \mathrm{M}}\right)\right|_{\mathfrak{U}_{n, M}}$ is $\sum \mathrm{Fil}^{s_{0}}\left(\mathbb{A}_{\text {cris, } \mathrm{M}}^{\nabla}\right) X_{1}^{\left[s_{1}\right]} \cdots X_{d}^{\left[s_{d}\right]}$ over all $s_{0}, \ldots, s_{d} \in \mathbb{N}$ such that $s_{0}+\cdots+s_{d} \geq r$.

We remark that the element $t$ is an element of Fil $^{1}\left(A_{\text {cris }}\right)$ and, hence, of Fil ${ }^{1}\left(\mathbb{A}_{\text {cris,M }}\right)\left(\bar{R}_{\mathcal{U}}\right)$ as well. Write $B_{\text {cris }}^{\nabla}\left(\bar{R}_{\mathcal{U}}\right)=A_{\text {cris }}^{\nabla}\left(\bar{R}_{\mathcal{U}}\right)\left[\frac{1}{t}\right]$ and $B_{\text {cris }}\left(\bar{R}_{\mathcal{U}}\right)=A_{\text {cris }}\left(\bar{R}_{\mathcal{U}}\right)\left[\frac{1}{t}\right]$. Note that since $t$ lies in $\operatorname{Ker}(\theta)$, it admits divided powers in $A_{\text {cris }}\left(\bar{R}_{\mathcal{U}}\right)$ so that $t^{p}=p ! t^{[p]}$ and $p$ is invertible in $B_{\text {cris }}\left(\bar{R}_{\mathcal{U}}\right)$. In particular the definition given here agrees with the one given in [Bri, Def. 6.1.11]. In [Bri, $\S 6.2 .1]$ decreasing filtrations $\left\{\mathrm{Fil}^{r} B_{\text {cris }}^{\nabla}\left(\bar{R}_{\mathcal{U}}\right)\right\}_{r \in \mathbb{Z}}$ on $B_{\text {cris }}^{\nabla}\left(\bar{R}_{\mathcal{U}}\right)$ and $\left\{\mathrm{Fil}^{r} B_{\text {cris }}\left(\bar{R}_{\mathcal{U}}\right)\right\}_{r \in \mathbb{Z}}$ on $B_{\text {cris }}\left(\bar{R}_{\mathcal{U}}\right)$ are defined. Then

Proposition 2.36. The filtrations $\left\{\mathrm{Fil}^{r}\left(\mathbb{A}_{\text {cris, } \mathrm{M}}^{\nabla}\right)\right\}_{r \in \mathbb{N}}$ and $\left\{\mathrm{Fil}^{r}\left(\mathbb{A}_{\text {cris, }, \mathrm{M}}\right)\right\}_{r \in \mathbb{N}}$ are decreasing, separated and exhaustive.

Let $\mathcal{U}$ be a small affine. Via the identifications $\mathbb{A}_{\text {cris, } \mathrm{M}}^{\nabla}\left(\bar{R}_{\mathcal{U}}\right) \cong A_{\text {cris }}^{\nabla}\left(\bar{R}_{\mathcal{U}}\right)$ and $\mathbb{A}_{\text {cris, } \mathrm{M}}\left(\bar{R}_{\mathcal{U}}\right) \cong$ $A_{\text {cris }}\left(\bar{R}_{\mathcal{U}}\right)$ given in 2.28 (resp. 2.34), we have for every $r \in \mathbb{Z}$ the identifications Fil $^{a} A_{\text {cris }}^{\nabla}\left(\bar{R}_{\mathcal{U}}\right)=$ $\mathrm{Fil}^{a} \mathbb{A}_{\text {cris, } \mathrm{M}}^{\nabla}\left(\bar{R}_{\mathcal{U}}\right)$ and $\mathrm{Fil}^{a} A_{\text {cris }}\left(\bar{R}_{\mathcal{U}}\right)=\mathrm{Fil}^{a} \mathbb{A}_{\text {cris, } \mathrm{M}}\left(\bar{R}_{\mathcal{U}}\right)$. In particular,

$$
\mathrm{Fil}^{r} B_{\text {cris }}^{\nabla}\left(\bar{R}_{\mathcal{U}}\right)=\sum_{a+b \geq r} t^{b} \mathrm{Fil}^{a} \mathbb{A}_{\text {cris, } \mathrm{M}}^{\nabla}\left(\bar{R}_{\mathcal{U}}\right)\left[p^{-1}\right] \text { and } \mathrm{Fil}^{r} B_{\text {cris }}\left(\bar{R}_{\mathcal{U}}\right)=\sum_{a+b \geq r} t^{b} \mathrm{Fil}^{a} \mathbb{A}_{\text {cris }, \mathrm{M}}\left(\bar{R}_{\mathcal{U}}\right)\left[p^{-1}\right]
$$

Proof. The first claim is clear. The filtrations on $B_{\text {cris }}^{\nabla}\left(\bar{R}_{\mathcal{U}}\right)$ and $B_{\text {cris }}\left(\bar{R}_{\mathcal{U}}\right)$ are defined in loc. cit. as the pull-back of the natural filtrations on $B_{\mathrm{dR}}\left(\bar{R}_{\mathcal{U}}\right)^{\nabla+}$ and $B_{\mathrm{dR}}\left(\bar{R}_{\mathcal{U}}\right)^{+}$via the inclusions $B_{\text {cris }}^{\nabla}\left(\bar{R}_{\mathcal{U}}\right) \subset$ $B_{\mathrm{dR}}\left(\bar{R}_{\mathcal{U}}\right)^{\nabla}$ and $B_{\text {cris }}\left(\bar{R}_{\mathcal{U}}\right) \subset B_{\mathrm{dR}}\left(\bar{R}_{\mathcal{U}}\right)$. In particular this induces a filtration on $A_{\text {cris }}^{\nabla}\left(\bar{R}_{\mathcal{U}}\right)$ by restriction. It is proved in [Bri, Pf. Prop. 6.2.1] that it coincides with the filtration induced by Fil $\mathbb{A}_{\text {cris }, \mathrm{M}}^{\nabla}$ via the identification $\mathbb{A}_{\text {cris, } \mathrm{M}}^{\nabla}\left(\bar{R}_{\mathcal{U}}\right)=A_{\text {cris }}^{\nabla}\left(\bar{R}_{\mathcal{U}}\right)$. By loc. cit. $A_{\text {cris }}^{\nabla}\left(\bar{R}_{\mathcal{U}}\right)$ maps to the ring $B_{\mathrm{dR}}\left(\bar{R}_{\mathcal{U}}\right)^{+}=B_{\mathrm{dR}}\left(\bar{R}_{\mathcal{U}}\right)^{\nabla+} \llbracket X_{1}, \ldots, X_{d} \rrbracket$ and due to [Bri, Prop. 5.2.5] we have Fil ${ }^{r}\left(B_{\mathrm{dR}}\left(\bar{R}_{\mathcal{U}}\right)^{+}\right)=$ $\sum_{s_{0}+\ldots s_{d}=r} \mathrm{Fil}^{s_{0}}\left(B_{\mathrm{dR}}\left(\bar{R}_{\mathcal{U}}\right)^{\nabla+}\right) X_{1}^{s_{1}} \cdots X_{d}^{s_{d}}$. Since $A_{\text {cris }}\left(\bar{R}_{\mathcal{U}}\right)=A_{\text {cris }}^{\nabla}\left(\bar{R}_{\mathcal{U}}\right)\left\{\left\langle X_{1}, \ldots, X_{d}\right\rangle\right\}$, also the filtration on $A_{\text {cris }}\left(\bar{R}_{\mathcal{U}}\right)$ induced from $B_{\text {cris }}\left(\bar{R}_{\mathcal{U}}\right)$ coincides with the filtration associated to Fil $\mathbb{A}_{\text {cris,M }}$ via the identification $\mathbb{A}_{\text {cris, } \mathrm{M}}\left(\bar{R}_{\mathcal{U}}\right)=A_{\text {cris }}\left(\bar{R}_{\mathcal{U}}\right)$. Since multiplication by $t$ induces a shift by -1 on Fil ${ }^{\bullet} B_{\text {cris }}^{\nabla}\left(\bar{R}_{\mathcal{U}}\right)$ and on Fil ${ }^{\bullet} B_{\text {cris }}\left(\bar{R}_{\mathcal{U}}\right)$ by [Bri, Prop. 5.2.1] and [Bri, Prop. 5.2.5] and since multiplication by $p$ on $B_{\mathrm{dR}}\left(\bar{R}_{\mathcal{U}}\right)$ is an isomorphism and preserves the filtration, the claim follows.

For every $i \in \mathbb{N}$ let $\Omega_{X / \mathcal{O}_{K}}^{i} \in \operatorname{Sh}\left(X^{\mathrm{et}}\right)$ be the sheaf of continuous Kähler differentials on the étale site of $X$ relative to $\mathcal{O}_{K}$. Then $v_{X, M}^{*}\left(\Omega_{X / \mathcal{O}_{K}}^{i}\right)$ is a locally free sheaf of $v_{X, M}^{*}\left(\mathcal{O}_{X}\right) \cong \mathcal{O}_{\mathfrak{X}_{M}}^{\text {un }}$ modules over $\mathfrak{X}_{M}$. The de Rham complex on $X$ defines a de Rham complex $v_{X, M}^{*}\left(\Omega_{X / \mathcal{O}_{K}}\right)$ on $\mathfrak{X}_{M}$. For every $n$ we get a complex $\mathbb{W}_{X, M, n} \otimes_{\mathcal{O}_{\mathfrak{X}_{M}}^{\text {un }}} v_{X, M}^{*}\left(\Omega_{X / \mathcal{O}_{K}}^{\bullet}\right)$ with $\mathbb{W}_{n, M}$-linear maps $\nabla^{i+1}: \mathbb{W}_{X, n, M} \otimes_{\mathcal{O}_{\mathfrak{x}_{M}}^{\text {un }}}$ $v_{X, M}^{*}\left(\Omega_{X / \mathcal{O}_{K}}^{i}\right) \rightarrow \mathbb{W}_{X, n, M} \otimes_{\mathcal{O}_{\mathfrak{X}_{M}}^{\text {un }}} v_{X, M}^{*}\left(\Omega_{X / \mathcal{O}_{K}}^{i+1}\right)$. 
Convention: In order to simplify the notation, for every sheaf of $\mathcal{O}_{\mathfrak{X}_{M}}^{\text {un }}$-modules $\mathcal{E}$ and any sheaf of $\mathcal{O}_{X}$-modules $\mathcal{M}$ we write $\mathcal{E} \otimes_{\mathcal{O}_{X}} \mathcal{M}$ for $\mathcal{E} \otimes_{\mathcal{O}_{\mathfrak{X}_{M}}^{\text {un }}} v_{X, M}^{*}(\mathcal{M})$.

Let $d$ be the relative dimension of $X$ over $\mathcal{O}_{K}$. Then we have

Proposition 2.37. The complex $\mathbb{W}_{X, n, M} \otimes_{\mathcal{O}_{X}} \Omega_{X / \mathcal{O}_{K}}^{\bullet}$ extends uniquely to a complex

$$
\mathbb{A}_{\text {cris }, \mathrm{M}} \stackrel{\nabla^{1}}{\longrightarrow} \mathbb{A}_{\text {cris }, \mathrm{M}} \otimes_{\mathcal{O}_{X}} \Omega_{X / \mathcal{O}_{K}}^{1} \stackrel{\nabla^{2}}{\longrightarrow} \mathbb{A}_{\text {cris }, \mathrm{M}} \otimes_{\mathcal{O}_{X}} \Omega_{X / \mathcal{O}_{K}}^{2} \longrightarrow \cdots \stackrel{\nabla^{d}}{\longrightarrow} \mathbb{A}_{\text {cris }, \mathrm{M}} \otimes_{\mathcal{O}_{X}} \Omega_{X / \mathcal{O}_{K}}^{d} \longrightarrow 0
$$

with the following property: for every $(\mathcal{U}, \mathcal{W}) \in \mathfrak{X}_{M}$, for $m, n$ and $i \in \mathbb{N}$ and for $x \in$ $\operatorname{Ker}\left(\theta_{X, n}\right)(\mathcal{U}, \mathcal{W}) \in \mathbb{A}_{\text {cris,n,M }}(\mathcal{U}, \mathcal{W})$ and $\omega \in \Omega_{\mathcal{U} / \mathcal{O}_{K}}^{i}$ we have $\nabla^{i+1}\left(x^{[m]} \otimes \omega\right)=x^{[m-1]} \otimes \nabla^{i+1}(x \omega)$. Furthermore

i. the sequence above is exact;

ii. the natural inclusion $\mathbb{A}_{\text {cris, } \mathrm{M}}^{\nabla} \subset \mathbb{A}_{\text {cris,M }}$ identifies $\operatorname{Ker}\left(\nabla^{1}\right)$ with $\mathbb{A}_{\text {cris,M }}^{\nabla}$;

iii. (Griffith's transversality) we have $\nabla\left(\mathrm{Fil}^{r}\left(\mathbb{A}_{\text {cris, } \mathrm{M}}\right)\right) \subset \mathrm{Fil}^{r-1}\left(\mathbb{A}_{\text {cris }, \mathrm{M}}\right) \otimes_{\mathcal{O}_{X}} \Omega_{X / \mathcal{O}_{K}}^{1}$ for every $r$;

iv. for every $r \in \mathbb{N}$ the sequence $0 \longrightarrow \mathrm{Fil}^{r} \mathbb{A}_{\text {cris,M }}^{\nabla} \longrightarrow \mathrm{Fil}^{r} \mathbb{A}_{\text {cris }, \mathrm{M}} \stackrel{\nabla^{1}}{\longrightarrow} \mathrm{Fil}^{r-1} \mathbb{A}_{\text {cris,M }} \otimes_{\mathcal{O}_{X}}$ $\Omega_{X / \mathcal{O}_{K}}^{1} \stackrel{\nabla^{2}}{\longrightarrow} \mathrm{Fil}^{r-2} \mathbb{A}_{\text {cris }, \mathrm{M}} \otimes_{\mathcal{O}_{X}} \Omega_{X / \mathcal{O}_{K}}^{2} \stackrel{\nabla^{3}}{\longrightarrow} \cdots$, with the convention that $\mathrm{Fil}{ }^{s} \mathbb{A}_{\text {cris }, \mathrm{M}}=\mathbb{A}_{\text {cris, }, \mathrm{M}}$ for $s<0$, is exact;

v. the connection $\nabla: \mathbb{A}_{\text {cris, } \mathrm{M}} \longrightarrow \mathbb{A}_{\text {cris,M }} \otimes_{\mathcal{O}_{X}} \Omega_{X / \mathcal{O}_{K}}^{1}$ is quasi-nilpotent;

vi. Let $\mathcal{U}$ be a small affine, choose parameters $T_{1}, \ldots, T_{d} \in R_{\mathcal{U}}^{\times}$and let $F_{\mathcal{U}}$ be the induced lift of absolute Frobenius to $R_{\mathcal{U}}$. Then Frobenius $\varphi_{\mathcal{U}}$ on $\left.\mathbb{A}_{\text {cris, }}\right|_{\mathcal{U}}$ is horizontal with respect to $\nabla_{\mathcal{U}}$ i. e., $\left.\nabla\right|_{\mathcal{U}} \circ \varphi_{\mathcal{U}}=\left.\left(\varphi_{\mathcal{U}} \otimes d F_{\mathcal{U}}\right) \circ \nabla\right|_{\mathcal{U}}$.

Proof. The uniqueness is clear. We have to prove that the formula defining $\nabla^{i}$ is well defined. By uniqueness it suffices to show it after passing to the subcategory $\mathfrak{U}_{n, M}$ where $\mathcal{U}$ is a small affine of $X^{\text {et }}$. Write $\mathbb{A}_{\text {cris, }, M}{\mid \mathfrak{U}_{n, M}} \cong \mathbb{A}_{\text {cris,n,M } M}^{\nabla}{\mid \mathfrak{U}_{n, M}}_{\mathfrak{U}}\left\langle X_{1}, \ldots, X_{d}\right\rangle$ as in 2.31. Then $\mathcal{O}_{\mathfrak{X}_{M}} \otimes_{\mathcal{O}_{X}} \Omega_{X / \mathcal{O}_{K}}^{1}$ restricted to $\mathfrak{U}_{M}$ is a free $\mathcal{O}_{\mathfrak{U}_{M}}$-module with basis $d T_{1}, \ldots, d T_{d}$ and the element $X_{i} \in \mathbb{W}_{X, n}\left(\mathfrak{U}_{n, M}\right)$ satisfies $\nabla\left(X_{i}\right)=1 \otimes d T_{i}$. In particular the complex above extends uniquely to a complex $\left.\mathbb{A}_{\text {cris, }, \mathrm{M}}\right|_{\mathfrak{U}_{n, M}} \otimes_{\mathcal{O}_{\mathcal{U}}} \Omega_{\mathcal{U}_{/} \mathcal{O}_{K}}$ characterized by the property that $\nabla\left(X_{i}^{[m]}\right)=X_{i}^{[m-1]} \otimes d T_{i}$.

Claims (i)-(vi) can be also checked after passing to $\mathfrak{U}_{n, M}$. Claims (ii) and (iii) follow from the formulae given above. Claims (i) and (iv) follow from the formulae and Poincarés lemma for the PD polynomial algebras ([BO, Proof of Thm. 6.12]). Claim (v) follows remarking that $\nabla\left(\partial / \partial T_{i}\right)^{p} \equiv 0$ modulo $p \mathbb{A}_{\text {cris, }}$.

Note that $F_{\mathcal{U}}\left(T_{i}\right)=T_{i}^{p}$ so that $\varphi_{\mathcal{U}}\left(X_{i}\right)=1 \otimes T_{i}^{p}-\widetilde{T}_{i}^{p} \otimes 1$. Hence $\varphi_{\mathcal{U}}\left(X_{i}^{[m]}\right)=\left(1 \otimes T_{i}^{p}-\right.$ $\left.\widetilde{T}_{i}^{p} \otimes 1\right)^{[m]}$. We compute $\nabla\left(\varphi_{\mathcal{U}}\left(X_{i}^{[m]}\right)\right)=\left(1 \otimes T_{i}^{p}-\widetilde{T}_{i}^{p} \otimes 1\right)^{[m-1]} \otimes \nabla\left(1 \otimes T_{i}^{p}-\widetilde{T}_{i}^{p} \otimes 1\right)=\left(1 \otimes T_{i}^{p}-\right.$ $\left.\widetilde{T}_{i}^{p} \otimes 1\right)^{[m-1]} \otimes d T_{i}^{p}=\left(\varphi_{\mathcal{U}} \otimes d F_{\mathcal{U}}\right) \nabla\left(X_{i}^{[m]}\right)$. This proves $(\mathrm{vi})$. 
We conclude this section with a variant of the constructions above considering Tate twists. For every integer $r$ define the inverse systems of sheaves of $\mathbb{A}_{\text {cris, }, \mathrm{M}^{-}}^{\prime} \nabla$ modules (resp. $\mathbb{A}_{\text {cris,n, }, \mathrm{M}^{-}}$ modules):

$$
\mathbb{A}_{\text {cris }, \mathrm{M}}^{\nabla}(r):=\left\{u_{X, M, *}\left(\mathbb{Z}_{p} / p^{n} \mathbb{Z}_{p}(r)\right) \otimes_{\mathbb{Z}_{p}} \mathbb{A}_{\text {cris,n,M }}^{\prime \nabla}\right\}_{n}
$$

and

$$
\mathbb{A}_{\text {cris }, \mathrm{M}}(r):=\left\{u_{X, M, *}\left(\mathbb{Z}_{p} / p^{n} \mathbb{Z}_{p}(r)\right) \otimes_{\mathbb{Z}_{p}} \mathbb{A}_{\text {cris,n,M }}^{\prime}\right\}_{n}
$$

For $i \geq 1$ define

$$
\nabla^{i-1}(r): \mathbb{A}_{\mathrm{cris}, \mathrm{M}}(r) \otimes_{\mathcal{O}_{X}} \Omega_{X / \mathcal{O}_{K}}^{i} \longrightarrow \mathbb{A}_{\mathrm{cris}, \mathrm{M}}(r) \otimes_{\mathcal{O}_{X}} \Omega_{X / \mathcal{O}_{K}}^{i+1}
$$

to be induced by the system of morphisms on $u_{X, M, *}\left(\mathbb{Z}_{p} / p^{n} \mathbb{Z}_{p}(r)\right) \otimes_{\mathbb{Z}_{p}} \mathbb{A}_{\text {cris,n,M }}^{\prime} \otimes_{\mathcal{O}_{X}} \Omega_{X / \mathcal{O}_{K}}^{i} \rightarrow$ $u_{X, M, *}\left(\mathbb{Z}_{p} / p^{n} \mathbb{Z}_{p}(r)\right) \otimes_{\mathbb{Z}_{p}} \mathbb{A}_{\text {cris }, \mathrm{n}, \mathrm{M}}^{\prime} \otimes_{\mathcal{O}_{X}} \Omega_{X / \mathcal{O}_{K}}^{i+1}$ given by $1 \otimes \nabla^{i-1}$. Put $\nabla(r):=\nabla^{1}(r)$.

We define an exhaustive, separated decreasing filtration on $\mathbb{A}_{\text {cris,M }}^{\nabla}(r)$ (resp. on $\left.\mathbb{A}_{\text {cris,M }}(r)\right)$ by inverse systems of sub-sheaves by setting

$$
\operatorname{Fil}^{i} \mathbb{A}_{\text {cris }, \mathrm{M}}^{\nabla}(r):=\left\{u_{X, M}^{*}\left(\mathbb{Z}_{p} / p^{n} \mathbb{Z}_{p}(r)\right) \otimes_{\mathbb{Z}_{p}} \operatorname{Fil}^{i-r} \mathbb{A}_{\text {cris,n,M }}^{\prime \nabla}(\mathcal{U}, \mathcal{W})\right\}_{n}
$$

and

$$
\operatorname{Fil}^{i} \mathbb{A}_{\text {cris }, \mathrm{M}}(r):=\left\{u_{X, M}^{*}\left(\mathbb{Z}_{p} / p^{n} \mathbb{Z}_{p}(r)\right) \otimes_{\mathbb{Z}_{p}} \operatorname{Fil}^{i-r} \mathbb{A}_{\text {cris,n,M }}^{\prime}(\mathcal{U}, \mathcal{W})\right\}_{n}
$$

for every $i \geq r$ and setting it to be $\mathbb{A}_{\text {cris,M }}^{\nabla}(r)$ (resp. $\left.\mathbb{A}_{\text {cris, }, \mathrm{M}}(r)\right)$ for $i \leq r$.

Recall that $p^{-1}=(p-1) ! \frac{t^{[p]}}{t^{p}} \in A_{\text {cris }} \cdot t^{-p}$. Thus, $p^{-r}$ lies in $A_{\text {cris }} t^{-p r}$ and, since it is invariant under $G_{M}$, it is a well defined element of $\left.\mathbb{A}_{\text {cris,n,M }}^{\prime} \nabla r\right)$ for every $n \in \mathbb{N}$. Define Frobenius $\varphi: \mathbb{A}_{\text {cris,M }}^{\prime} \nabla$

$$
u_{X, M}^{*}\left(\mathbb{Z}_{p} / p^{n} \mathbb{Z}_{p}(r)\right) \otimes_{\mathbb{Z}_{p}} \mathbb{A}_{\text {cris,n,M }}^{\prime} \nabla
$$

given by $a \otimes b \mapsto a \otimes p^{-r} \varphi(b)$. Assume that $\mathcal{U}$ is a small affine, choose parameters $T_{1}, \ldots, T_{d} \in R_{\mathcal{U}}^{\times}$ and let $F_{\mathcal{U}}$ be the induced lift of absolute Frobenius to $R_{\mathcal{U}}$. Then using the same formula we get a Frobenius $\varphi_{\mathcal{U}}:\left.\left.\mathbb{A}_{\text {cris,M }}^{\prime}(r)\right|_{\mathcal{U}} \rightarrow \mathbb{A}_{\text {cris, }, \mathrm{M}}^{\prime}(p r)\right|_{\mathcal{U}}$ which is horizontal with respect to the connection $\nabla(r) \mathcal{u}$

Lemma 2.38. The filtrations $\left\{\mathrm{Fil}^{i}\left(\mathbb{A}_{\text {cris, }, \mathrm{M}}^{\nabla}(r)\right)\right\}_{r \in \mathbb{N}}$ and $\left\{\mathrm{Fil}^{i}\left(\mathbb{A}_{\text {cris }, \mathrm{M}}(r)\right)\right\}_{i \in \mathbb{N}}$ are decreasing, separated and exhaustive.

Let $\mathcal{U}$ be a small affine of $X^{\mathrm{et}}$. Then

$\operatorname{Fil}^{i}\left(\mathbb{A}_{\text {cris }, \mathrm{M}}^{\nabla}(r)\right)\left(\bar{R}_{\mathcal{U}}\right) \cong \mathrm{Fil}^{i-r} A_{\text {cris }}^{\nabla}\left(\bar{R}_{\mathcal{U}}\right) \cdot t^{r}$ and $\mathrm{Fil}^{i}\left(\mathbb{A}_{\text {cris }, \mathrm{M}}(r)\right)\left(\bar{R}_{\mathcal{U}}\right) \cong \mathrm{Fil}^{i-r} A_{\text {cris }}\left(\bar{R}_{\mathcal{U}}\right) \cdot t^{r}$.

The analogue of claims (i)-(vi) of 2.37 hold for the connection $\nabla(r)$ and the system of sheaves $\mathbb{A}_{\text {cris, }, \mathrm{M}}^{\nabla}(r)$ and $\mathbb{A}_{\text {cris }, \mathrm{M}}(r)$.

Proof. The first and second statements follow from proposition 2.36. The last statement follows from the definition and proposition 2.37. 


\subsection{The ind-sheaves $\mathbb{B}_{\text {cris }}^{\nabla}$ and $\mathbb{B}_{\text {cris }}$.}

Generalities on inductive systems. Let $\mathcal{A}$ be an abelian category. We denote by $\operatorname{Ind}(\mathcal{A})$, called the category of inductive systems of objects of $\mathcal{A}$, the category whose objects are $\left(A_{i}, \gamma_{i}\right)_{i \in \mathbb{Z}}$ with $A_{i}$ object of $\mathcal{A}$ and $\gamma_{i}: A_{i} \rightarrow A_{i+1}$ morphism in $\mathcal{A}$ for every $i \in \mathbb{Z}$. Given an integer $N \in \mathbb{Z}$ a morphism $f: \underline{A}:=\left(A_{i}, \gamma_{i}\right)_{i \in \mathbb{Z}} \longrightarrow \underline{B}:=\left(B_{j}, \delta_{j}\right)_{j \in \mathbb{Z}}$ of degree $N$ is a system of morphisms $f_{i}: A_{i} \rightarrow B_{i+N}$ for $i \in \mathbb{Z}$ such that $\delta_{i+N} \circ f_{i}=f_{i+1} \circ \gamma_{i}$. Since $\mathcal{A}$ is an additive category the set of morphisms of degree $N$ form an abelian group with the zero map, the sum of two functions and the inverse of a function defined componentwise. Given a morphism $f=\left(f_{i}\right)_{i \in \mathbb{Z}}: \underline{A} \longrightarrow \underline{B}$ of degree $N$ we get a morphism of degree $N+1$ given by $\left(\delta_{i+N} \circ f_{i}\right)_{i \in \mathbb{Z}}$. This defines a group homomorphism from the morphisms $\operatorname{Hom}^{N}(\underline{A}, \underline{B})$ of degree $N$ to the morphisms $\operatorname{Hom}^{N+1}(\underline{A}, \underline{B})$ of degree $N+1$. We define the group of morphisms $f:\left(A_{i}, \gamma_{i}\right)_{i \in \mathbb{Z}} \longrightarrow\left(B_{j}, \delta_{j}\right)_{j \in \mathbb{Z}}$ in $\operatorname{Ind}(\mathcal{A})$ to be the inductive limit $\lim _{N \in \mathbb{Z}} \operatorname{Hom}^{N}(\underline{A}, \underline{B})$ with respect to the transition maps just defined.

Given any such morphism $f$ we let $\operatorname{Ker}(f)$ be the inductive system $\left(\operatorname{Ker}\left(f_{i}\right)\right)_{i \in \mathbb{Z}}$ with transition morphisms defined by the $\gamma_{i}^{\prime}$ 's. We let Coker $(f)$ be the inductive system $\left(\operatorname{Coker}\left(f_{i-N}\right)_{i \in \mathbb{Z}}\right.$ with transition morphisms induced by the $\delta_{i}$ 's. One verifies that with these definitions the category $\operatorname{Ind}(\mathcal{A})$ is an abelian category. Note that we have a natural functor

$$
\mathcal{A} \longrightarrow \operatorname{Ind}(\mathcal{A})
$$

sending $A$ to the inductive system $(A, \mathrm{Id})_{i \in \mathbb{Z}}$ which is exact and fully faithful.

Assume furthermore that $\mathcal{A}$ is a tensor category. Given objects $\underline{A}:=\left(A_{i}, \gamma_{i}\right)_{i \in \mathbb{Z}}$ and $\underline{B}:=$ $\left(B_{j}, \delta_{j}\right)_{j \in \mathbb{Z}}$ in $\operatorname{Ind}(\mathcal{A})$ we define $\underline{A} \otimes \underline{B}$ to be the inductive system $\left(A_{i} \otimes B_{i}, \gamma_{i} \otimes \delta_{i}\right)_{i \in \mathbb{Z}}$. In this way $\operatorname{Ind}(\mathcal{A})$ is endowed with the structure of a tensor category so that the functor $\mathcal{A} \longrightarrow \operatorname{Ind}(\mathcal{A})$ is a morphism of tensor categories. By abuse of notation given an object $B \in \mathcal{A}$ we write $\underline{A} \otimes B$ for the inductive system $\underline{A} \otimes \underline{B}$ with $\underline{B}=(B, \mathrm{Id})$.

Let $\mathcal{B}$ be an abelian category in which direct limits of inductive systems indexed by $\mathbb{Z}$ exist. Consider the induced functor

$$
\lim _{\rightarrow} \operatorname{Ind}(\mathcal{B}) \longrightarrow \mathcal{B}
$$

Suppose we are given $\delta$-functors $T^{n}: \mathcal{B} \rightarrow \mathcal{A}$ with $n \in \mathbb{N}$. Define

$$
\lim _{\rightarrow} T^{n}: \operatorname{Ind}(\mathcal{A}) \longrightarrow \mathcal{B}
$$

as the composite of the functor $\operatorname{Ind}(\mathcal{A}) \rightarrow \operatorname{Ind}(\mathcal{B})$, given by $\left(A_{i}\right)_{i n \mathbb{Z}} \mapsto\left(T^{n}\left(A_{i}\right)\right)_{i \in \mathbb{Z}}$ and of the functor $\lim$.

Lemma 2.39. If $\lim _{\rightarrow}$ is left exact then the functors $\lim _{\rightarrow} T^{n}$, for varying $n \in \mathbb{N}$, define a $\delta$-functor. If $\left(T^{n}\right)_{n \in \mathbb{N}}$ is universal then also $\left(\lim _{\rightarrow} T^{n}\right)_{n \in \mathbb{N}}$ is universal.

Proof. Due to the universal property of direct limits the functor lim is always right exact. Thus

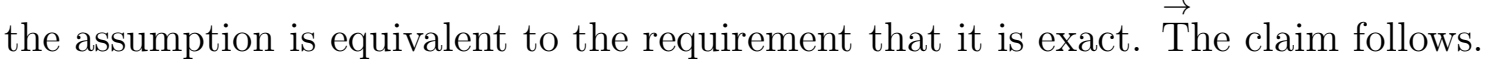


Remark 2.40. One can relax the definition of a morphism in $\operatorname{Ind}(\mathcal{A})$. Consider a non-decreasing function $\alpha: \mathbb{Z} \rightarrow \mathbb{Z}$. Given objects $\underline{A}:=\left(A_{i}, \gamma_{i}\right)_{i \in \mathbb{Z}}$ and $\underline{B}:=\left(B_{j}, \delta_{j}\right)_{j \in \mathbb{Z}}$, we define a morphism $f: \underline{A} \rightarrow \underline{B}$ of type $\alpha$ to be a collection of morphisms $f_{i}: A_{i} \rightarrow B_{\alpha(i)}$ such that $f_{i+1} \circ \gamma_{i}=$ $\prod_{\alpha(i) \leq j<\alpha(i+1)} \delta_{j} \circ f_{i}$. We denote by $\operatorname{Hom}^{\alpha}(\underline{A}, \underline{B})$ the group of homomorphisms of type $\alpha$. We say that two morphisms $f$ and $g$ of type $\alpha$ (resp. $\beta$ ) are equivalent if there exists $N \in \mathbb{N}$ such that $f_{i}$ composed with $B_{\alpha(i)} \rightarrow B_{\max (\alpha(i), \beta(i))+N}$ and $g_{i}$ composed with $B_{\beta(i)} \rightarrow B_{\max (\alpha(i), \beta(i))+N}$ coincide. One checks that this defines an equivalence relation. We define a morphism $\underline{A} \rightarrow \underline{B}$ to be a class of morphisms with respect to this equivalence relation. The morphisms in the more restrictive sense given before inject into this new class of morphisms. Since this complicates the notation we will work mainly with the previous more restrictive notion.

Recall that given an integer $r$ the sheaf $\mathbb{A}_{\text {cris,n,M }}^{\nabla}(r)$ is characterized by the property that for every small affine open $\mathcal{U} \in X^{\text {et }}$ its localization $\mathbb{A}_{\text {cris,n,M }}^{\nabla}(r)\left(\bar{R}_{\mathcal{U}}\right)$ is the group $\mathbb{A}_{\text {cris,n,M }}^{\nabla}\left(\bar{R}_{\mathcal{U}}\right)$ with action of $\mathcal{G}_{\mathcal{U}, M}$ twisted by the $r$-th power of the cyclotomic character. Let $t:=\log [\epsilon] \in A_{\text {cris }}$ (see section [1.2). Fix integers $r \geq s$. For every $\mathcal{U}$ as above we have a map $\mathbb{A}_{\text {cris,n,M }}(s)\left(\bar{R}_{\mathcal{U}}\right) \longrightarrow$ $\mathbb{A}_{\text {cris,n,M }}^{\nabla}(r)\left(\bar{R}_{\mathcal{U}}\right)$ of $\mathbb{A}_{\text {cris,n,M }}^{\nabla}\left(\bar{R}_{\mathcal{U}}\right)$-module sending $1 \mapsto 1 \otimes t^{[r-s]}$. Since they are equivariant with respect to the action of $\mathcal{G}_{\mathcal{U}, M}$, these maps for varying $\mathcal{U}$ arise from a unique morphism

$$
j_{r, s}: \mathbb{A}_{\text {cris }, \mathrm{n}, \mathrm{M}}^{\nabla}(s) \rightarrow \mathbb{A}_{\text {cris }, \mathrm{n}, \mathrm{M}}^{\nabla}(r) .
$$

They are compatible for varying $n \in \mathbb{N}$ and define a morphism of continuous sheaves $j_{r, s}: \mathbb{A}_{\text {cris, }}^{\nabla}(s) \rightarrow$ $\mathbb{A}_{\text {cris,M }}^{\nabla}(r)$. Define $\iota_{r, s}:=(r-s) ! j_{r, s}$. It follows from the construction that $j_{r, s}$, and hence $\iota_{r, s}$, sends $\mathrm{Fil}^{n} \mathbb{A}_{\text {cris,M }}^{\nabla}(s)$ to $\mathrm{Fil}^{n} \mathbb{A}_{\text {cris }, \mathrm{M}}^{\nabla}(r)$ for every $n \in \mathbb{Z}$.

Define $\mathbb{B}_{\text {cris,M }}^{\nabla}$ in Ind $\left(\operatorname{Sh}\left(\mathfrak{X}_{M}\right)^{\mathbb{N}}\right)$ to be the inductive system of continuous sheaves having $\mathbb{A}_{\text {cris,M }}^{\nabla}(-r)$ in degree $r$ with transition maps given by $\iota_{r-1, r}$. Analogously for every $n \in \mathbb{Z}$ let $\mathrm{Fil}^{n} \mathbb{B}_{\text {cris,M }}^{\nabla}$ in Ind $\left(\operatorname{Sh}\left(\mathfrak{X}_{M}\right)^{\mathbb{N}}\right)$ be the inductive system of continuous sheaves having Fil $^{n} \mathbb{A}_{\text {cris,M }}^{\nabla}(-r)$ in degree $r$ with transition maps induced by $\iota_{r-1, r}$. By construction it is a sub-object of $\mathbb{B}_{\text {cris, }, \mathrm{M}}^{\nabla}$. The Frobenius morphisms on $\varphi: \mathbb{A}_{\text {cris,M }}^{\prime} \nabla$ (r) $\left.\rightarrow \mathbb{A}_{\text {cris,M }}^{\prime} \nabla r\right)$ are compatible for varying $r \in \mathbb{Z}$ with $\iota_{r-1, r}$. Using the more general notion of a morphism of inductive systems given in 2.40 it induces a morphism $\varphi: \mathbb{B}_{\text {cris }, \mathrm{M}}^{\nabla} \rightarrow \mathbb{B}_{\text {cris }, \mathrm{M}}^{\nabla}$ in Ind $\left(\operatorname{Sh}\left(\mathfrak{X}_{M}\right)^{\mathbb{N}}\right)$ sending $\mathrm{Fil}^{r} \mathbb{B}_{\text {cris, } \mathrm{M}}^{\nabla} \longrightarrow \mathrm{Fil}^{r+p} \mathbb{B}_{\text {cris }, \mathrm{M}}^{\nabla}$.

Similarly, we define the continuous sheaves $\mathbb{A}_{\text {cris, } M}(r)$. As before we get the inductive systems $\mathbb{B}_{\text {cris, } M}$ and $\mathrm{Fil}^{n} \mathbb{B}_{\text {cris, } \mathrm{M}}$, for $n \in \mathbb{Z}$, in ind $\left(\operatorname{Sh}\left(\mathfrak{X}_{M}\right)^{\mathbb{N}}\right)$ as the inductive system of continuous sheaves having $\mathbb{A}_{\text {cris,M }}(-r)$ (resp. Fil ${ }^{n} \mathbb{A}_{\text {cris }, M}(-r)$ ) in degree $r$. Assume that $\mathcal{U}$ is a small affine. Then Frobenius $\varphi_{\mathcal{U}}:\left.\left.\mathbb{A}_{\text {cris,M }}^{\prime}(r)\right|_{\mathcal{U}} \rightarrow \mathbb{A}_{\text {cris,M }}^{\prime}(p r)\right|_{\mathcal{U}}$ induces, in the more general framework of 2.40, a morphism $\varphi_{\mathcal{U}}:\left.\left.\mathbb{B}_{\text {cris, } M}\right|_{\mathcal{U}} \rightarrow \mathbb{B}_{\text {cris,M }}\right|_{\mathcal{U}}$ in Ind $\left(\operatorname{Sh}\left(\mathfrak{U}_{M}^{\bullet}\right)^{\mathbb{N}}\right)$.

We remark that the morphisms $j_{s+p, s}$ for varying $s \in \mathbb{Z}$ define a morphism $j$ on $\mathrm{Fil}^{n} \mathbb{B}_{\text {cris, } \mathrm{M}}^{\nabla}$, $\mathbb{B}_{\text {cris, },}^{\nabla}, \mathrm{Fil}^{n} \mathbb{B}_{\text {cris,M }}$ and $\mathbb{B}_{\text {cris }, \mathrm{M}}$ such that $p ! j$ is the identity in the category of inductive systems. We deduce that multiplication by $p$ is an isomorphism on all the objects above.

For notational convention put $\mathrm{Fil}^{-\infty} \mathbb{B}_{\text {cris, } \mathrm{M}}^{\nabla}=\mathbb{B}_{\text {cris, } \mathrm{M}}^{\nabla}$ and similarly without $\nabla$. As explained before, the localization functor on $\operatorname{Sh}\left(\mathfrak{X}_{M}\right)^{\mathbb{N}}, \mathcal{F} \mapsto \mathcal{F}\left(\bar{R}_{\mathcal{U}}\right)$, extends to a functor on Ind $\left(\operatorname{Sh}\left(\mathfrak{X}_{M}\right)^{\mathbb{N}}\right)$. Put Fil ${ }^{-\infty} B_{\text {cris }}^{\nabla}\left(\bar{R}_{\mathcal{U}}\right)=B_{\text {cris }}^{\nabla}\left(\bar{R}_{\mathcal{U}}\right)$ and similarly without $\nabla$. Summarizing and using the results of the previous section we get :

Lemma 2.41. (1) Multiplication by $p$ is an isomorphism on $\mathrm{Fil}^{n} \mathbb{B}_{\text {cris, }, \mathrm{M}}^{\nabla}, \mathbb{B}_{\text {cris, }, \mathrm{M}}^{\nabla}, \mathrm{Fil}^{n} \mathbb{B}_{\text {cris }, \mathrm{M}}$ and $\mathbb{B}_{\text {cris, }, \mathrm{M}}$. 
(2) For every $r \in \mathbb{Z} \cup\{-\infty\}$ we have an exact sequence of inductive systems

$$
0 \longrightarrow \mathrm{Fil}^{r} \mathbb{B}_{\text {cris, } \mathrm{M}}^{\nabla} \longrightarrow \mathrm{Fil}^{r} \mathbb{B}_{\text {cris }, \mathrm{M}} \stackrel{\nabla^{1}}{\longrightarrow} \mathrm{Fil}^{r-1} \mathbb{B}_{\text {cris, } \mathrm{M}} \otimes_{\mathcal{O}_{X}} \Omega_{X / \mathcal{O}_{K}}^{1} \stackrel{\nabla^{2}}{\longrightarrow} \mathrm{Fil}^{r-2} \mathbb{B}_{\text {cris }, \mathrm{M}} \otimes_{\mathcal{O}_{X}} \Omega_{X / \mathcal{O}_{K}}^{2} \cdots
$$

Let $\mathcal{U}$ be a small affine, choose parameters $T_{1}, \ldots, T_{d} \in R_{\mathcal{U}}^{\times}$and let $F_{\mathcal{U}}$ be the induced lift of absolute Frobenius to $R_{\mathcal{U}}$. Then,

(3) Frobenius $\varphi_{\mathcal{U}}$ on $\left.\mathbb{B}_{\text {cris, }, M}\right|_{\mathcal{U}}$ is horizontal with respect to $\left.\nabla\right|_{\mathcal{U}}$ and induces Frobenius on $\mathbb{B}_{\text {cris, },}^{\nabla} \mid \mathcal{U}$.

(4) $\mathbb{B}_{\text {cris, } \mathrm{M}}^{\nabla}\left(\bar{R}_{\mathcal{U}}\right) \cong B_{\text {cris }}^{\nabla}\left(\bar{R}_{\mathcal{U}}\right)$ and $\mathbb{B}_{\text {cris, } \mathrm{M}}\left(\bar{R}_{\mathcal{U}}\right) \cong B_{\text {cris }}\left(\bar{R}_{\mathcal{U}}\right)$. Furthermore, Fil ${ }^{i}\left(\mathbb{B}_{\text {cris,M }}^{\nabla}\right)\left(\bar{R}_{\mathcal{U}}\right) \cong$ $\mathrm{Fil}^{i} B_{\text {cris }}^{\nabla}\left(\bar{R}_{\mathcal{U}}\right)$ and $\mathrm{Fil}^{i}\left(\mathbb{B}_{\text {cris, } \mathrm{M}}\right)\left(\bar{R}_{\mathcal{U}}\right) \cong \mathrm{Fil}^{i} B_{\text {cris }}\left(\bar{R}_{\mathcal{U}}\right)$ for every $i \in \mathbb{Z}$.

\subsection{The fundamental exact sequence}

Following [Fo, $§ 5.3 .6]$ put $\mathrm{Fil}_{p}^{r} A_{\text {cris }}=\left\{x \in \mathrm{Fil}^{r} A_{\text {cris }} \mid \varphi(x) \in p^{r} A_{\text {cris }}\right\}$ for every $r \in \mathbb{N}$. Let $\frac{\varphi}{p^{r}}: \mathrm{Fil}_{p}^{r} A_{\text {cris }} \rightarrow A_{\text {cris }}$ be the induced map. Note that $p^{r} \mathrm{Fil}^{r} A_{\text {cris }} \subset \mathrm{Fil}^{r} A_{\text {cris }} \subset \mathrm{Fil}^{r} A_{\text {cris }}$. For every $n$ and $r \in \mathbb{N}$ define the sheaf

$$
\operatorname{Fil}_{p}^{r} \mathbb{A}_{\text {cris }, \mathrm{n}, \overline{\mathrm{K}}}^{\prime}:=\left(\mathrm{Fil}_{p}^{r} A_{\text {cris }} / p^{n} \mathrm{Fil}_{p}^{r} A_{\text {cris }}\right) \otimes_{W_{n}} \mathbb{W}_{n, \bar{K}}
$$

For $r=0$ it coincides with $\mathbb{A}_{\text {cris,n, }, \bar{K}}^{\prime}$ thanks to lemma 2.26 (c). Since $\mathbb{W}_{n, \bar{K}}$ is flat as a sheaf of $W_{n}$-modules by corollary $2.22, \mathrm{Fil}_{p}^{r} \mathbb{A}_{\text {cris,n, }, \overline{\mathrm{K}}}^{\prime} \nabla$ defines a subsheaf of $\mathrm{Fil}_{p}^{0} \mathbb{A}_{\text {cris,n, }, \overline{\mathrm{K}}}^{\prime}=\mathbb{A}_{\text {cris }, \mathrm{n}, \overline{\mathrm{K}}}^{\prime}$. Let

$$
\frac{\varphi}{p^{r}}: \operatorname{Fil}_{p}^{r} \mathbb{A}_{\text {cris }, \mathrm{n}, \overline{\mathrm{K}}}^{\prime \nabla} \longrightarrow \mathbb{A}_{\text {cris }, \mathrm{n}, \overline{\mathrm{K}}}^{\prime}
$$

be the morphism defined by $\frac{\varphi}{p^{r}}$ on $\mathrm{Fil}_{p}^{r} A_{\text {cris }} / p^{n} \mathrm{Fil}_{p}^{r} A_{\text {cris }}$ and by $\varphi$ on $\mathbb{W}_{n, \bar{K}}$. Let $\mathrm{Fil}_{p}^{r} \mathbb{A}_{\text {cris,n }+1, \overline{\mathrm{K}}}^{\prime} \rightarrow$ $\mathrm{Fil}_{p}^{r} \mathbb{A}_{\text {cris,n, }, \overline{\mathrm{K}}}^{\prime}$ be the morphism defined by reduction modulo $p^{n}$ on $\mathrm{Fil}_{p}^{r} A_{\text {cris }} / p^{n+1} \mathrm{Fil}_{p}^{r} A_{\text {cris }}$ and $r_{n+1, \bar{K}}: \mathbb{W}_{n+1, \bar{K}} \rightarrow \mathbb{W}_{n, \bar{K}}$. Put $\mathrm{Fil}_{p}^{r} \mathbb{A}_{\text {cris, } \overline{\mathrm{K}}}^{\prime}$ to be the associated inverse system of sheaves. Write $\frac{\varphi}{p^{r}}$ for the induced morphism $\operatorname{Fil}_{p}^{r} \mathbb{A}_{\text {cris }, \overline{\mathrm{K}}}^{\prime} \nabla \underset{\mathbb{A}_{\text {cris }, \overline{\mathrm{K}}}^{\prime} \nabla}{ }$.

Proposition 2.42. Assume we are in the formal case. Then:

(1) The sequence

$$
0 \longrightarrow \mathbb{Z} / p^{n} \mathbb{Z} t^{\{r\}} \longrightarrow \mathrm{Fil}_{p}^{r} \mathbb{A}_{\text {cris }, \mathrm{n}, \overline{\mathrm{K}}}^{\prime} \stackrel{1-\frac{\varphi}{p^{r}}}{\longrightarrow} \mathbb{A}_{\text {cris, }, \overline{\mathrm{K}}}^{\prime} \longrightarrow 0
$$

is exact.

(2) The morphism of continuous sheaves $\mathrm{Fil}_{p}^{r} \mathbb{A}_{\text {cris }, \overline{\mathrm{K}}}^{\prime} \rightarrow \mathrm{Fil}^{r} \mathbb{A}_{\text {cris, } \overline{\mathrm{K}}}^{\prime \nabla}$ is an isomorphism in $\operatorname{Sh}\left(\mathfrak{X}_{M}\right)_{\mathbb{Q}_{p}}$.

Proof. We start with the proof of (2). It follows from 2.22 and 2.26 that $\mathrm{Fil}^{r}\left(A_{\text {cris }} / p^{n} A_{\text {cris }}\right) \otimes_{W_{n}}$ $\mathbb{A}_{\text {cris }, \mathrm{n}, \overline{\mathrm{K}}}^{\prime} \rightarrow \mathrm{Fil}^{r} \mathbb{A}_{\text {cris, }, \overline{\mathrm{K}}}^{\prime}$ is an isomorphism. We then get natural maps $p^{r} \mathrm{Fil}^{r} \mathbb{A}_{\text {cris }, \mathrm{n}-\mathrm{r}, \overline{\mathrm{K}}}^{\prime} \rightarrow$ $\mathrm{Fil}_{p}^{r} \mathbb{A}_{\text {cris, }, \overline{\mathrm{K}}}^{\prime \nabla} \rightarrow \mathrm{Fil}^{r} \mathbb{A}_{\text {cris }, \mathrm{n}-\mathrm{r}, \overline{\mathrm{K}}}^{\prime} \nabla$ inducing morphisms of continuous sheaves

$$
p^{r} \mathrm{Fil}^{r} \mathbb{A}_{\text {cris }, \overline{\mathrm{K}}}^{\prime \nabla} \rightarrow \operatorname{Fil}_{p}^{r} \mathbb{A}_{\text {cris }, \overline{\mathrm{K}}}^{\prime} \rightarrow \operatorname{Fil}^{r} \mathbb{A}_{\text {cris }, \overline{\mathrm{K}}}^{\prime \nabla}
$$


This proves (2).

For the proof of (1) we proceed as in the proof of [T, Thm. A3.26]. Following [Fo, §5.3.1] define $I^{[s]} A_{\text {cris }}:=\left\{x \in A_{\text {cris }} \mid \varphi^{n}(x) \in\right.$ Fil $\left.^{s} A_{\text {cris }} \forall n \in \mathbb{N}\right\}$. For every $m \in \mathbb{N}$ write $m=q(m)(p-$ $1)+r(m)$ with $0 \leq r(m)<p-1$. Let $t^{\{m\}}:=t^{r(m)} \cdot\left(\frac{t^{p-1}}{p}\right)^{[q(m)]}$. It is proven in loc. cit. that $I^{[s]} A_{\text {cris }}$ is the closure for the $p$-adic topology of the $A_{\text {inf }}^{+}$-module generated by the elements $t^{\{s\}}$ for $s \geq r$. Furthermore $A_{\text {cris }} / I^{[s]} A_{\text {cris }}$ is $p$-torsion free by [Fo, Prop. 5.3.5]. In particular the decreasing filtration $I^{[s]} A_{\text {cris }} \cap \mathrm{Fil}_{p}^{r} A_{\text {cris }}$ on $\mathrm{Fil}_{p}^{r} A_{\text {cris }}$ for $s \in \mathbb{N}$, has torsion free graded quotients. Its reduction modulo $p^{n}$ injects into $\mathrm{Fil}_{p}^{r} A_{\text {cris }} / p^{n} \mathrm{Fil}_{p}^{r} A_{\text {cris }}$ and defines a decreasing filtration on the latter. Since $\mathbb{W}_{n, \bar{K}}$ is flat as a sheaf of $W_{n}$-modules by 2.22 taking $\otimes_{W_{n}} \mathbb{W}_{n, \bar{K}}$ we get a decreasing

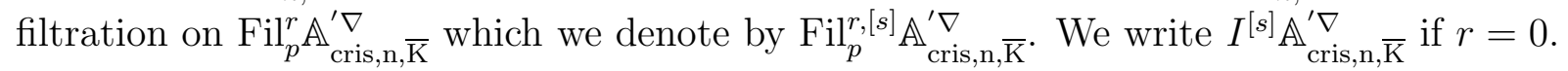

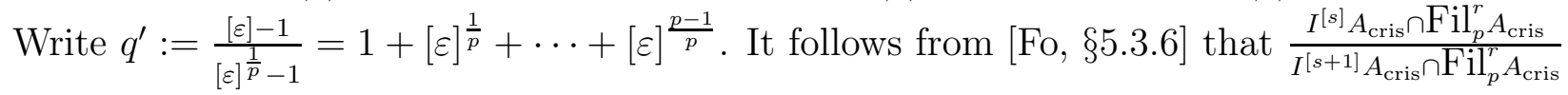
is $p$-torsion free and it is generated as $A_{\text {inf }}^{+}$-module by the element $\left(q^{\prime}\right)^{r-s} t^{\{s\}}$ for $0 \leq s<r$ and by $t^{\{s\}}$ for $s \geq r$. Since $\varphi\left(q^{\prime}\right) \in p A_{\text {cris }}$, by [Fo, $\left.\S 5.2 .9\right]$, the map $\frac{\varphi}{p^{r}}$ sends $I^{[s]} A_{\text {cris }} \cap \operatorname{Fil}_{p}^{r} A_{\text {cris }}$ to $I^{[s]} A_{\text {cris }}$ so that $1-\frac{\varphi}{p^{r}}$ sends $I^{[s]} A_{\text {cris }} \cap \mathrm{Fil}_{p}^{r} A_{\text {cris }}$ to $I^{[s]} A_{\text {cris }}$. We deduce that the morphism $1-\frac{\varphi}{p^{r}}$ sends $\mathrm{Fil}_{p}^{r,[s]} \mathbb{A}_{\text {cris }, \mathrm{n}, \overline{\mathrm{K}}}^{\prime}$ to $I^{[s]} b A_{\text {cris }, \mathrm{n}, \overline{\mathrm{K}}}^{\prime}$. The conclusion follows from 2.43 ,

Lemma 2.43. The morphism $1-\frac{\varphi}{p^{r}}$ induces isomorphisms

$$
\mathrm{Fil}_{p}^{r,[r+1]} \mathbb{A}_{\text {cris }, \mathrm{n}, \overline{\mathrm{K}}}^{\prime \nabla} \longrightarrow I^{[r+1]} b A_{\text {cris }, \mathrm{n}, \overline{\mathrm{K}}}^{\prime \nabla}
$$

and

$$
\frac{\mathrm{Fil}_{p}^{r,[s]} \mathbb{A}_{\text {cris }, \mathrm{n}, \overline{\mathrm{K}}}^{\prime} \nabla}{\mathrm{Fil}_{p}^{r,[s+1]} \mathbb{A}_{\text {cris }, \mathrm{n}, \overline{\mathrm{K}}}^{\prime}} \longrightarrow \frac{I^{[s]} b A_{\text {cris }, \mathrm{n}, \overline{\mathrm{K}}}^{\prime} \nabla}{I^{[s+1]} b A_{\text {cris }, \mathrm{n}, \overline{\mathrm{K}}}^{\prime}}
$$

for $0 \leq s<r$ and an exact sequence

$$
0 \longrightarrow \mathbb{Z} / p^{n} \mathbb{Z} \cdot t^{\{r\}} \longrightarrow \frac{\mathrm{Fil}_{p}^{r,[r]} \mathbb{A}_{\text {cris }, \mathrm{n}, \overline{\mathrm{K}}}^{\prime} \nabla}{\mathrm{Fil}_{p}^{r,[r+1]} \mathbb{A}_{\text {cris }, \mathrm{n}, \overline{\mathrm{K}}}^{\prime}} \stackrel{1-\frac{\varphi}{p^{r}}}{\longrightarrow} \frac{I_{\text {cris,n, } \overline{\mathrm{K}}}^{[r]} b A^{\prime} \nabla}{I^{[r+1]} b A_{\text {cris }, \mathrm{n}, \overline{\mathrm{K}}}^{\prime}} \longrightarrow 0
$$

Proof. By construction we have $\mathrm{Fil}_{p}^{r,[r+1]} \mathbb{A}_{\text {cris }, \overline{\mathrm{K}}}^{\prime}=I^{[r+1]} b A_{\text {cris, }, \overline{\mathrm{K}}}^{\prime}$ so that the operator $1-\frac{\varphi}{p^{r}}$ is $1-p \cdot \frac{\varphi}{p^{r+1}}$ which is unipotent and hence an isomorphism. This proves the first assertion.

It follows from $\left[\mathrm{Fo}\right.$, Prop. 5.1.3 \& Rmk. 5.3.2] that $I^{[s]} A_{\text {cris }} \cap A_{\text {inf }}^{+}=([\varepsilon]-1)^{s} A_{\text {inf }}^{+}$and that

$$
A_{\text {inf }}^{+} /([\varepsilon]-1) A_{\text {inf }}^{+} \longrightarrow I^{[s]} A_{\text {cris }} / I^{[s+1]} A_{\text {cris }}, \quad x \mapsto x \cdot t^{\{s\}}
$$

is an isomorphism. In particular this isomorphism induces the isomorphisms

$$
A_{\text {inf }}^{+} /\left([\varepsilon]^{\frac{1}{p}}-1\right) A_{\text {inf }}^{+} \longrightarrow \frac{I^{[s]} A_{\text {cris }} \cap \mathrm{Fil}_{p}^{r} A_{\text {cris }}}{I^{[s+1]} A_{\text {cris }} \cap \mathrm{Fil}_{p}^{r} A_{\text {cris }}}, \quad x \mapsto x\left(q^{\prime}\right)^{r-s} t^{\{s\}}
$$

for $0 \leq s<r$ and the isomorphism

$$
A_{\text {inf }}^{+} /([\varepsilon]-1) A_{\text {inf }}^{+} \longrightarrow \frac{I^{[s]} A_{\text {cris }} \cap \mathrm{Fil}_{p}^{r} A_{\text {cris }}}{I^{[s+1]} A_{\text {cris }} \cap \mathrm{Fil}_{p}^{r} A_{\text {cris }}}=I^{[s]} A_{\text {cris }} / I^{[s+1]} A_{\text {cris }}, \quad x \mapsto x t^{\{s\}}
$$


for $s \geq r$. It follows from $[\mathrm{Fo}, \S 5.2 .9]$ that $\left(1-\frac{\varphi}{p^{r}}\right)\left(\left(q^{\prime}\right)^{r-s} t^{\{s\}}\right) \equiv t^{\{s\}} \bmod I^{[s+1]} A_{\text {cris }}$ for every $0 \leq s \leq r$. We then deduce by base changing via the flat extension $W_{n} \rightarrow \mathbb{W}_{n, \bar{K}}$ that for $0 \leq s \leq r$ the following diagram

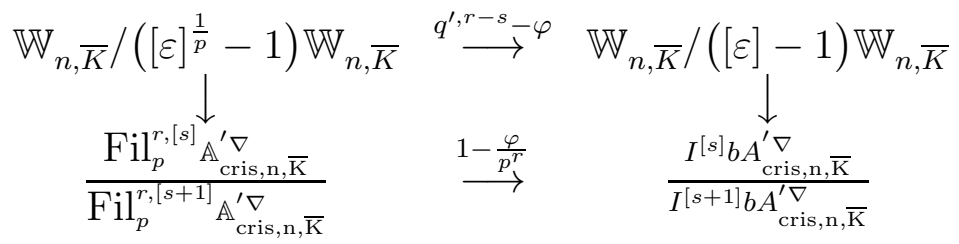

is commutative and that the vertical arrows are isomorphisms. The second claim of the lemma follows remarking that the top horizontal morphism is the sum of a nilpotent map, given by multiplication by $q^{\prime, r-s}$ and the map $-\varphi$ which is an isomorphism due to 2.22. Similarly for $s=r$ the following diagram is commutative with vertical arrows isomorphisms

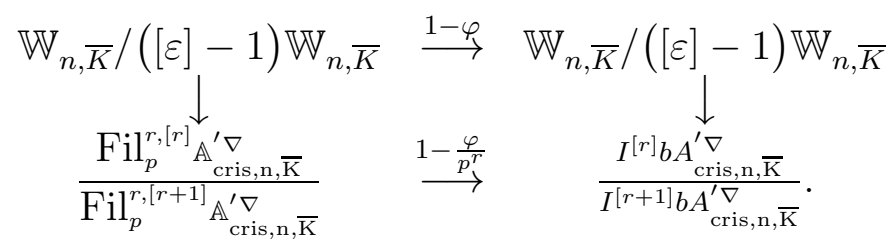

The last assertion follows remarking that the top horizontal arrow is surjective with kernel $\mathbb{Z} / p^{n} \mathbb{Z}$ due to corollary 2.22 ,

Define $\mathrm{Fil}_{p}^{r} \mathbb{A}_{\text {cris }, \overline{\mathrm{K}}}^{\nabla}(m)$ as the system $\left\{\left(\mathbb{Z}_{p} / p^{n} \mathbb{Z}_{p}(r)\right) \otimes_{\mathbb{Z}_{p}} \mathrm{Fil}_{p}^{i-r} \mathbb{A}_{\text {cris }, \mathrm{n}, \mathrm{M}}^{\prime}\right\}_{n}$. Let $\mathrm{Fil}_{p}^{r} \mathbb{B}_{\text {cris }, \overline{\mathrm{K}}}^{\nabla}$ be the inductive system of continuous sheaves $\operatorname{Fil}_{p}^{r} \mathbb{A}_{\text {cris }, \overline{\mathrm{K}}}^{\nabla}(m)$.

Since multiplication by $p$ is an isomorphism on $\mathrm{Fil}^{r} \mathbb{B}_{\text {cris }, \overline{\mathrm{K}}}^{\nabla}$ by 2.41 it follows from proposition 2.42 that it coincides with $\mathrm{Fil}^{r} \mathbb{B}_{\text {cris }, \overline{\mathrm{K}}}^{\nabla}$. The morphism $1-\varphi: \mathbb{A}_{\text {cris }, \overline{\mathrm{K}}}^{\prime}(-r) \rightarrow \mathbb{A}_{\text {cris }, \overline{\mathrm{K}}}^{\prime}(-p r)$ induces a morphism of inductive systems $1-\varphi: \mathbb{B}_{\text {cris }, \overline{\mathrm{K}}}^{\nabla} \longrightarrow \mathbb{B}_{\text {cris }, \overline{\mathrm{K}}}^{\nabla}$. Since multplication by $t$ is an isomorphism on $\mathbb{B}_{\text {cris, } \overline{\mathrm{K}}}^{\nabla}$ by 3.1 we deduce from proposition 2.42 the exact sequence

$$
0 \longrightarrow \mathbb{Q}_{p} \longrightarrow \mathrm{Fil}^{0} \mathbb{B}_{\text {cris }, \overline{\mathrm{K}}}^{\nabla} \stackrel{1-\varphi}{\longrightarrow} \mathbb{B}_{\text {cris }, \overline{\mathrm{K}}}^{\nabla} \longrightarrow 0
$$

We then get the following commutative diagram with exact rows, called the fundamental diagram of sheaves:

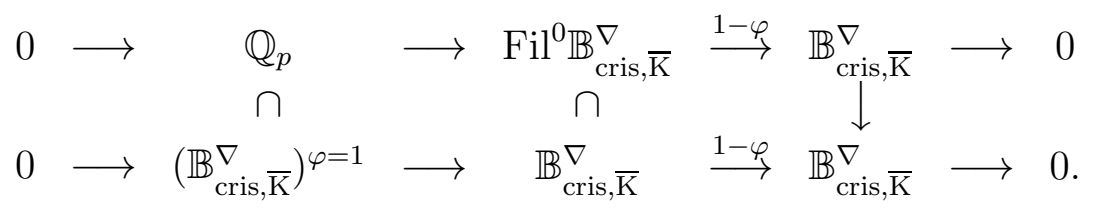

\section{The crystalline comparison isomorphism.}

\subsection{Crystalline étale sheaves.}

In this section we assume that $X$ is defined over $\mathcal{O}_{K}=\mathbb{W}(k)$ so that the sheaf $\mathbb{A}_{\text {cris, }, \mathrm{M}}$ is defined. Recall that we have natural morphisms of sites $u_{M}: \mathfrak{X}_{M} \longrightarrow X_{M}^{\text {et }}$, given by $(\mathcal{U}, \mathcal{W}) \mapsto \mathcal{W}$, and 
$v_{M}: X^{\text {et }} \longrightarrow \mathfrak{X}_{M}$ given by $\mathcal{U} \mapsto\left(\mathcal{U}, \mathcal{U}^{\text {rig }}\right)$. If $\mathbb{L}$ is a sheaf on $X_{M}^{\text {et }}$, to ease the notation we simply write $\mathbb{L}$ for $u_{M, *}(\mathbb{L})$. The aim of this section is to introduce the so called "crystalline $\mathbb{Q}_{p}$-adic sheaves" on $X_{M}^{\text {et }}$. As explained in proposition 3.7 the definition amounts to a sheaf theoretic generalization of the usual notion of crystalline representation, due to Fontaine, in the relative setting. We show in 3.7 that this notion coincides with the notion of "locally crystalline representations" introduced by [Bri] in the relative setting. We will prove in lemma 3.14 that it is also equivalent to Faltings' notion of associated sheaves. Contrary to these alternative definitions which are checked on small enough open affines the present definition has the advantage of being purely sheaf theoretic.

$\mathbb{Q}_{p}$-adic sheaves. By a $p$-adic sheaf $\mathbb{L}$ on $X_{M}^{\text {et }}$ we mean a system $\left\{\mathbb{L}_{n}\right\} \in \operatorname{Sh}\left(X_{M}^{\text {et }}\right)^{\mathbb{N}}$ such that $\mathbb{L}_{n}$ is a locally constant and locally free of finite rank étale sheaf of $\mathbb{Z} / p^{n} \mathbb{Z}$-modules and $\mathbb{L}_{n}=$ $\mathbb{L}_{n+1} / p^{n} \mathbb{L}_{n+1}$ for every $n \in \mathbb{N}$. Given two $p$-adic sheaves $\mathbb{L}:=\left\{\mathbb{L}_{n}\right\}$ and $\mathcal{M}:=\left\{\mathcal{M}_{n}\right\}$ define $\mathbb{L} \otimes_{\mathbb{Z}_{p}} \mathcal{M}:=\left\{\mathbb{L}_{n} \otimes_{\mathbb{Z} / p^{n} \mathbb{Z}} \mathcal{M}_{n}\right\}_{n}$ and $\underline{\operatorname{Hom}}(\mathbb{L}, \mathcal{M}):=\left\{\underline{\operatorname{Hom}}\left(\mathbb{L}_{n}, \mathcal{M}_{n}\right)\right\}_{n}$. Put $\mathbf{1}=\mathbb{Z}_{p}$ to be the sheaf $\left\{\mathbb{Z} / p^{n} \mathbb{Z}\right\}_{n}$ with $\mathbb{Z} / p^{n} \mathbb{Z}$ the constant sheaf. This defines a structure of abelian tensor category on $p$-adic sheaves on $X_{M}^{\text {et }}$. Define $\mathbb{Z}_{p}(1)$ to be the sheaf $\left\{\mu_{p^{n}}\right\}_{n}$ of $p$-power roots of unity. For every $m \in \mathbb{N}$ define $\mathbb{Z}_{p}(m)$ to be the $m$-fold tensor product of $\mathbb{Z}_{p}(1)$. For $m \leq 0$ put $\mathbb{Z}_{p}(m):=\underline{\operatorname{Hom}}\left(\mathbb{Z}_{p}(-m), \mathbb{Z}_{p}\right)$. For $m \in \mathbb{Z}$ and $\mathbb{L}$ a $p$-adic sheaf denote $\mathbb{L}(m):=\mathbb{L} \otimes_{\mathbb{Z}_{p}} \mathbb{Z}_{p}(m)$.

Define $\operatorname{Sh}\left(X_{M}^{\mathrm{et}}\right)_{\mathbb{Q}_{p}}$ to be the full subcategory of Ind $\left(\operatorname{Sh}\left(X_{M}^{\mathrm{et}}\right)^{\mathbb{N}}\right)$ (see $\left.₫ 2.8\right)$ consisting of inductive systems of the form $(\mathbb{L})_{i \in \mathbb{Z}}$ where $\mathbb{L}$ is a $p$-adic étale sheaf and the transition maps $\mathbb{L} \rightarrow \mathbb{L}$ are given by multiplication by $p$. It inherits from the category of $p$-adic sheaves on $X_{M}^{\text {et }}$ the structure of an abelian tensor category.

Let $\mathbb{L}=\left\{\mathbb{L}_{n}\right\}$ be a $p$-adic étale sheaf. By definition for every $(\mathcal{U}, \mathcal{W}) \in \mathfrak{X}_{M}$ we have $u_{X, M, *}\left(\mathbb{L}_{n}\right)(\mathcal{U}, \mathcal{W})=\mathbb{L}_{n}(\mathcal{W})$. Since $\mathbb{L}_{n}$ is a locally constant sheaf of finite abelian groups there exists $\mathcal{W} \in \mathcal{U}_{\mathrm{M}, \text { fet }}$ such that for every morphism $\left(\mathcal{U}^{\prime}, \mathcal{W}^{\prime}\right) \rightarrow(\mathcal{U}, \mathcal{W})$ in $\mathfrak{X}_{M}$ the map $\mathbb{L}_{n}\left(\mathcal{W}^{\prime}\right) \rightarrow$ $\mathbb{L}_{n}(\mathcal{W})$ is a bijection. In particular $u_{X, M, *}\left(\mathbb{L}_{n}\right)$ is locally constant on $\mathfrak{X}_{M}$ and $u_{X, M, *}$ is fully faithful. Similarly if we extend $u_{X, M, *}$ to inductive systems of inverse systems of sheaves we get a fully faithful morphism $u_{X, M, *}: \operatorname{Sh}\left(X_{M}^{\mathrm{et}}\right)_{\mathbb{Q}_{p}} \longrightarrow \operatorname{Ind}\left(\operatorname{Sh}\left(\mathfrak{X}_{M}^{\mathrm{et}}\right)^{\mathbb{N}}\right)$. We simply write $\mathbb{L}_{n}$ for $u_{M, *}\left(\mathbb{L}_{n}\right)$ and $\mathbb{L}$ for the inverse system of sheaves $\left\{u_{M, *}\left(\mathbb{L}_{n}\right)\right\}_{n}$

If $\mathcal{U}=\operatorname{Spf}\left(R_{\mathcal{U}}\right)$ is affine connected then the localization $\mathbb{L}_{n}\left(\bar{R}_{\mathcal{U}}\right)$ as defined in 2.2 is given by a free $\mathbb{Z}_{p} / p^{n} \mathbb{Z}$-module with continuous action of $\mathcal{G}_{\mathcal{U}_{M}}$ which we denote by $V_{\mathcal{U}}\left(\mathbb{L}_{n}\right)$. Write $V_{\mathcal{U}}(\mathbb{L})=\lim _{\infty \leftarrow n} V_{\mathcal{U}}\left(\mathbb{L}_{n}\right)$

The categories $\operatorname{Mod}\left(\mathfrak{X}_{M}\right)_{\mathbb{B}_{\text {cris }}^{\nabla}}$ and $\operatorname{Mod}\left(\mathfrak{X}_{M}\right)_{\mathbb{B}_{\text {cris }}}$. Denote by $\operatorname{Mod}\left(\mathfrak{X}_{M}\right)_{\mathbb{A}_{\text {cris }}^{\nabla}}\left(\operatorname{resp} \cdot \operatorname{Mod}\left(\mathfrak{X}_{M}\right)_{\mathbb{A}_{\text {cris }}}\right)$ the following category. The objects are systems $\left\{\mathcal{M}_{n}\right\}_{n} \in \operatorname{Sh}\left(\mathfrak{X}_{M}\right)^{\mathbb{N}}$ with $\mathcal{M}_{n}$ a sheaf of $\mathbb{A}_{\text {cris,n,M}}^{\prime} \nabla^{-}$ modules (resp. $\mathbb{A}_{\text {cris }, \mathrm{M}, \mathrm{n}}^{\prime}$-modules). Given objects $\mathcal{M}$ and $\mathcal{M}^{\prime}$ the morphisms are $\operatorname{Hom}_{\mathbb{A}_{\text {cris }, \mathrm{M}} \nabla}\left(\mathcal{M}, \mathcal{M}^{\prime}\right)$ (resp. $\left.\operatorname{Hom}_{\mathbb{A}_{\text {cris }, M}}\left(\mathcal{M}, \mathcal{M}^{\prime}\right)\right)$ i. e. the subset of $\operatorname{Hom}_{\operatorname{Sh}\left(\mathfrak{X}_{M}\right)^{\mathbb{N}}}\left(\mathcal{M}, \mathcal{M}^{\prime}\right)$ which are by definition compatible systems of homomorphisms $\left\{f_{n}: \mathcal{M}_{n} \rightarrow \mathcal{M}_{n}^{\prime}\right\}_{n \in \mathbb{N}}$ commuting with the underlying

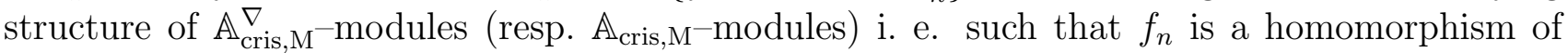
$\mathbb{A}_{\text {cris,n, }, M^{\prime}}^{\prime}$ modules (resp. $\mathbb{A}_{\text {cris, }, \mathrm{M}^{-}}^{\prime}$-modules) for every $n \in \mathbb{N}$.

Define the sheaf $\underline{\operatorname{Hom}}_{\mathbb{A}_{\text {cris }, M}^{\nabla}}\left(\mathcal{M}, \mathcal{M}^{\prime}\right)$ (resp. $\left.\underline{\operatorname{Hom}}_{\mathbb{A}_{\text {cris }, M}}\left(\mathcal{M}, \mathcal{M}^{\prime}\right)\right)$ in $\operatorname{Mod}\left(\mathfrak{X}_{M}\right)_{\mathbb{A}_{\text {cris }}}$ (respectivey in $\left.\operatorname{Mod}\left(\mathfrak{X}_{M}\right)_{A_{\text {cris }}}\right)$ associated to the pre-sheaf whose sections at $(\mathcal{U}, \mathcal{W}) \in \mathfrak{X}_{M}$ consist of the 
group

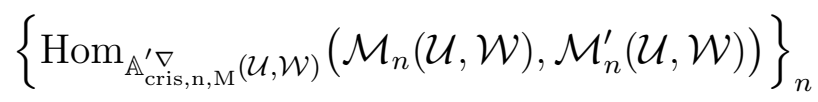

respectively

$$
\left\{\operatorname{Hom}_{\mathbb{A}_{\text {cris }, \mathrm{n}, \mathrm{M}}^{\prime}}(\mathcal{U}, \mathcal{W})\left(\mathcal{M}_{n}(\mathcal{U}, \mathcal{W}), \mathcal{M}_{n}^{\prime}(\mathcal{U}, \mathcal{W})\right)\right\}_{n}
$$

Define $\mathcal{M} \otimes_{\mathbb{A}_{\text {cris }, \mathrm{M}}^{\nabla}} \mathcal{M}^{\prime}$ (resp. $\left.\mathcal{M} \otimes_{\mathbb{A}_{\text {cris }, \mathrm{M}}} \mathcal{M}^{\prime}\right)$ to be the sheaf in $\operatorname{Mod}\left(\mathfrak{X}_{M}\right)_{A_{\text {cris }}^{\nabla}}$ (respectively in

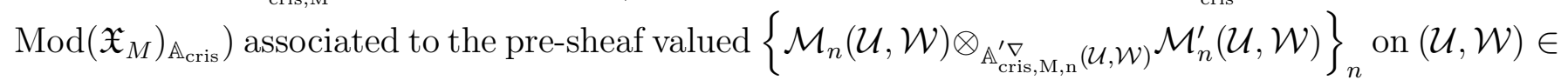
$\mathfrak{X}$ (respectively $\left.\left\{\mathcal{M}_{n}(\mathcal{U}, \mathcal{W}) \otimes_{\mathbb{A}_{\text {cris,n,M }}^{\prime}(\mathcal{U}, \mathcal{W})} \mathcal{M}_{n}^{\prime}(\mathcal{U}, \mathcal{W})\right\}_{n}\right)$. Define 1 to be the element $\mathbb{A}_{\text {cris }}$ (respectively $\left.\mathbb{A}_{\text {cris }}\right)$. With these structures both categories $\operatorname{Mod}\left(\mathfrak{X}_{M}\right)_{\mathbb{A}_{\text {cris }}}$ and $\operatorname{Mod}\left(\mathfrak{X}_{M}\right)_{\mathbb{A}_{\text {cris }}}$ are abelian tensor categories. Given any object $\mathcal{N}$ we write $\mathcal{N}(r)$ to be $\mathcal{N} \otimes_{\mathbb{A}_{\text {cris }, \mathrm{M}}} \mathbb{A}_{\text {cris,M }} \nabla(r)$ (respectively $\mathcal{N} \otimes_{\mathbb{A}_{\text {cris }, \mathrm{M}}} \mathbb{A}_{\text {cris,M }}(r)$.

Define $\operatorname{Mod}\left(\mathfrak{X}_{M}\right)_{\mathbb{B}_{\text {cris }}^{\nabla}}\left(\operatorname{resp} \cdot \operatorname{Mod}\left(\mathfrak{X}_{M}\right)_{\mathbb{B}_{\text {cris }}}\right)$ to be the full subcategory of $\operatorname{Ind}\left(\operatorname{Mod}\left(\mathfrak{X}_{M}\right)_{\mathbb{A}_{\text {cris }}^{\nabla}}\right)$ (respectively $\operatorname{Ind}\left(\operatorname{Mod}\left(\mathfrak{X}_{M}\right)_{\mathbb{A}_{\text {cris }}}\right)$ ) consisting of objects of the form $(\mathcal{M}(-r))_{r \in \mathbb{Z}}$ with $\mathcal{M}$ a fixed object of $\operatorname{Mod}\left(\mathfrak{X}_{M}\right)_{\mathbb{A}_{\text {cris }}}\left(\operatorname{resp} \cdot \operatorname{Mod}\left(\mathfrak{X}_{M}\right)_{\mathbb{A}_{\text {cris }}}\right)$ and the transition morphisms $\iota_{\mathcal{M}, r, s}: \mathcal{M}(s) \rightarrow \mathcal{M}(r)$ are induced by the morphisms $\iota_{r, s}: \mathbb{A}_{\text {cris }, \mathrm{M}}^{\nabla}(s) \rightarrow \mathbb{A}_{\text {cris,M }}^{\nabla}(r)$ (and similarly for $\mathbb{A}_{\text {cris,M }}$ ) defined in 92.8. Remark that this object is simply the tensor product $\mathcal{M} \otimes_{\mathbb{A}_{\text {cris }}} \mathbb{B}_{\text {cris }}^{\nabla}$ (resp. $\mathcal{M} \otimes_{\mathbb{A}_{\text {cris }}} \mathbb{B}_{\text {cris }}$ ) defined in $\$ 2.8$, Given objects $\mathcal{M}$ and $\mathcal{N}$ we denote by $\operatorname{Hom}_{\mathbb{B}_{\text {cris }}^{\nabla}}(\mathcal{M}, \mathcal{N})\left(\operatorname{resp} \operatorname{Hom}_{\mathbb{B}_{\text {cris }}^{\nabla}}(\mathcal{M}, \mathcal{N})\right)$ the group of homomorphisms in this category. These categories inherit from $\operatorname{Mod}\left(\mathfrak{X}_{M}\right)_{\mathbb{A}_{\text {cris }}}$ and $\operatorname{Mod}\left(\mathfrak{X}_{M}\right)_{\mathbb{A}_{\text {cris }}}$ the structures of tensor categories.

Consider objects $\mathcal{M}=\left\{\mathcal{M}_{a}\right\}_{a \in \mathbb{N}}$ and $\mathcal{N}=\left\{\mathcal{N}_{a}\right\}_{a \in \mathbb{N}}$ in $\operatorname{Mod}\left(\mathfrak{X}_{M}\right)_{\mathbb{A}_{\text {cris }}^{\nabla}}$ or in $\operatorname{Mod}\left(\mathfrak{X}_{M}\right)_{\mathbb{A}_{\text {cris }}}$. Given integers $m$ and $n$ and a morphism $f: \mathcal{M}(m) \longrightarrow \mathcal{N}(m+n)$ in $\operatorname{Mod}\left(\mathfrak{X}_{M}\right)_{\mathbb{A}_{\text {cris }}}$ (respectively $\left.\operatorname{Mod}\left(\mathfrak{X}_{M}\right)_{\mathbb{A}_{\text {cris }}}\right)$ we define a morphism of inductive systems $\left(f_{i}: \mathcal{M}(i) \longrightarrow \mathcal{N}(i+n)\right)_{i \in \mathbb{Z}}$ identifying

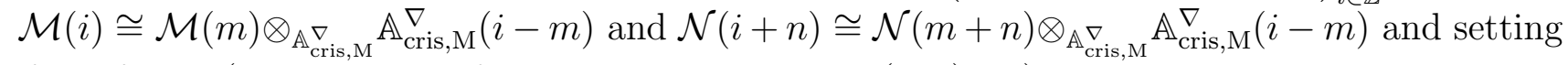
$f_{i}:=f \otimes \operatorname{Id}$ (and similarly if we have objects in $\operatorname{Mod}\left(\mathfrak{X}_{M}\right)_{\mathbb{A}_{\text {cris }}}$ ).

Lemma 3.1. The maps above define group isomorphisms

$$
\lim _{s, r \in \mathbb{Z}} \operatorname{Hom}_{\mathbb{A}_{\text {cris }, \mathrm{M}}^{\nabla}}(\mathcal{M}(s), \mathcal{N}(r)) \longrightarrow \operatorname{Hom}_{\mathbb{B}_{\text {cris }}^{\nabla}}(\mathcal{M}, \mathcal{N})
$$

Here, the direct limits on the left hand side is taken via the maps $\operatorname{Hom}_{\mathbb{A}_{\text {cris, }}}(\mathcal{M}(s), \mathcal{N}(r)) \rightarrow$ Hom $_{\mathbb{A}_{\text {cris, }, \mathrm{M}}}\left(\mathcal{M}\left(s^{\prime}\right), \mathcal{N}\left(r^{\prime}\right)\right)$ given by $f \mapsto \iota_{\mathcal{N}, r, r^{\prime}} \circ f \circ \iota_{\mathcal{M}, s^{\prime}, s}$ for integers $s^{\prime} \geq s$ and $r \geq r^{\prime}$. Similarly we get an isomorphisms

$$
\lim _{s, r \in \mathbb{Z}} \operatorname{Hom}_{\mathbb{A}_{\text {cris }, \mathrm{M}}}(\mathcal{M}(s), \mathcal{N}(r)) \longrightarrow \operatorname{Hom}_{\mathbb{B}_{\text {cris }}}(\mathcal{M}, \mathcal{N})
$$

Let $f \in \operatorname{Hom}_{\mathbb{B}_{\text {cris }}^{\text {ris }}}(\mathcal{M}, \mathcal{N})$ (resp. in $\left.\operatorname{Hom}_{\mathbb{B}_{\text {cris }}}(\mathcal{M}, \mathcal{N})\right)$ induced by a morphism $f_{m, n}: \mathcal{M}(m) \rightarrow$ $\mathcal{N}(n)$ in $\operatorname{Mod}\left(\mathfrak{X}_{M}\right)_{\mathbb{A}_{\text {cris }}}^{\nabla}$ (resp. $\left.\operatorname{Mod}\left(\mathfrak{X}_{M}\right)_{\mathbb{A}_{\text {cris }}}\right)$ for some $m$ and $n \in \mathbb{Z}$. The following are equivalent:

1) $f$ is an isomorphism;

2) there are $r$ and $s \in \mathbb{N}$ and a map $h_{r, s}: \mathcal{N}(n+r) \longrightarrow \mathcal{M}(m-s)$ such that $f_{m, n}(s) \circ h_{r, s}$ is $\iota_{\mathcal{N}, n+r, n-s}: \mathcal{N}(n+r) \rightarrow \mathcal{N}(n-s)$ and $h_{r, s} \circ f_{m, n}(+r)$ is $\iota_{\mathcal{M}, m+r, m-s} \mathcal{M}(m+r) \rightarrow \mathcal{M}(m-s)$; 
3) there exists $N \in \mathbb{N}$ such that for every small affine $\mathcal{U} \in X^{\text {et }}$ and every $a \in \mathbb{N}$ the map $\mathcal{M}_{a}(m)\left(\bar{R}_{\mathcal{U}}\right) \rightarrow \mathcal{N}_{a}(n)\left(\bar{R}_{\mathcal{U}}\right)$ induced by $f_{m, n}$ has kernel and cokernel annihilated by $t^{N}$.

Furthermore multiplication by $t$ is an isomorphism in $\operatorname{Mod}\left(\mathfrak{X}_{M}\right)_{\mathbb{B}_{\text {cris }}^{\nabla}}\left(\operatorname{resp} . \operatorname{Mod}\left(\mathfrak{X}_{M}\right)_{\mathbb{B}_{\text {cris }}}\right)$.

Proof. The first claim follows from the definitions and is left to the reader. The last claim follows from 2.41 remarking that any object is of the form $\mathcal{F} \otimes_{\mathbb{A}_{\text {cris }}^{\nabla}} \mathbb{B}_{\text {cris }}^{\nabla}$ (resp. $\left.\mathcal{F} \otimes_{\mathbb{A}_{\text {cris }}} \mathbb{B}_{\text {cris }}\right)$.

The equivalence of (1) and (2) is clear. Note that the morphism $\mathcal{M}(m)\left(\bar{R}_{\mathcal{U}}\right) \rightarrow \mathcal{N}(n)\left(\bar{R}_{\mathcal{U}}\right)$ is a morphism of $A_{\text {cris }}$-modules so that (3) makes sense.

$(2) \Longrightarrow(3)$ Note that $\mathcal{M}(h)\left(\bar{R}_{\mathcal{U}}\right)=\mathcal{M}\left(\bar{R}_{\mathcal{U}}\right)$ and $\mathcal{N}(h)\left(\bar{R}_{\mathcal{U}}\right)=\mathcal{N}\left(\bar{R}_{\mathcal{U}}\right)$ as $A_{\text {cris }}\left(\bar{R}_{\mathcal{U}}\right)$-modules for every $h \in \mathbb{Z}$ (only the Galois action of $\mathcal{G}_{\mathcal{U}, M}$ is different). Via these identifications the maps $\mathcal{N}(n+r)\left(\bar{R}_{\mathcal{U}}\right) \rightarrow \mathcal{N}(n-s)\left(\bar{R}_{\mathcal{U}}\right)$ and $\mathcal{M}(m+r)\left(\bar{R}_{\mathcal{U}}\right) \rightarrow \mathcal{M}(m-s)\left(\bar{R}_{\mathcal{U}}\right)$ are multiplication by $t^{s+r}$.

$(3) \Longrightarrow(2)$ Write $\mathcal{M}=\left\{\mathcal{M}_{a}\right\}_{a \in \mathbb{N}}$ and $\mathcal{N}:=\left\{\mathcal{N}_{b}\right\}_{b \in \mathbb{N}}$. Consider the map $\iota_{\mathcal{N}, n+2 N, n+N}: \mathcal{N}(n+$ $2 N) \rightarrow \mathcal{N}(n+N)$. By assumption for every small affine $\mathcal{U} \in \mathfrak{X}_{M}$ and every $a \in \mathbb{N}$ the image of the induced map on localizations $\mathcal{N}_{a}(n+2 N)\left(\bar{R}_{\mathcal{U}}\right) \rightarrow \mathcal{N}_{a}(n+N)\left(\bar{R}_{\mathcal{U}}\right)$ is zero in the cokernel of $f_{a, \mathcal{U}}(N): \mathcal{M}_{a}(m+N)\left(\bar{R}_{\mathcal{U}}\right) \rightarrow \mathcal{N}_{a}(n+N)\left(\bar{R}_{\mathcal{U}}\right)$ induced by $f_{m, n}$. Hence it factors via the image $\operatorname{Im}\left(f_{a, \mathcal{U}}(N)\right)$ of $f_{a, \mathcal{U}}(N)$. The map $\operatorname{Im}\left(f_{a, \mathcal{U}}(N)\right) \rightarrow \operatorname{Im}\left(f_{a, \mathcal{U}}\right)$ is multiplication by $t^{N}$ and hence factors uniquely via $\mathcal{M}_{a}(m)\left(\bar{R}_{\mathcal{U}}\right)$ by assumption. Thus the map $\mathcal{N}_{a}(n+2 N)\left(\bar{R}_{\mathcal{U}}\right) \rightarrow$ $\mathcal{N}_{a}(n)\left(\bar{R}_{\mathcal{U}}\right)$ obtained from $\iota_{\mathcal{N}, n+2 N, n}$ by localization factors uniquely via $f_{a, \mathcal{U}}: \mathcal{M}_{a}(m)\left(\bar{R}_{\mathcal{U}}\right) \rightarrow$ $\mathcal{N}_{a}(n)\left(\bar{R}_{\mathcal{U}}\right)$. By uniqueness this factorization is $\mathcal{G}_{\mathcal{U}, M^{-}}$equivariant and compatible for varying $\mathcal{U}$ 's and $a$ 's. In particular it provides a map $\left\{h_{a, 2 N, 0}: \mathcal{N}_{a}(n+2 N) \longrightarrow \mathcal{M}_{a}(m)\right\}_{a \in \mathbb{N}}$ with the required properties.

Let $\mathcal{U} \in X^{\text {et }}$ be a small affine with Galois group $\mathcal{G}_{\mathcal{U}, M}$. As explained in $\$ 2.8$ the localization functor $\operatorname{Sh}\left(\mathfrak{X}_{M}\right)^{\mathbb{N}} \rightarrow \operatorname{Rep}_{\mathcal{G}_{\mathcal{U}, M}} \mathcal{F} \mapsto \mathcal{F}\left(\bar{R}_{\mathcal{U}}\right)$ extend to a localization functor $\operatorname{Ind}\left(\operatorname{Sh}\left(\mathfrak{X}_{M}\right)^{\mathbb{N}}\right) \longrightarrow$ $\operatorname{Rep}_{\mathcal{G}_{\mathcal{U}, M}}$ which we denote by $\mathcal{F} \mapsto \mathcal{F}\left(\bar{R}_{\mathcal{U}}\right)$. Restricting it to the categories $\operatorname{Mod}\left(\mathfrak{X}_{M}\right)_{\mathbb{B}_{\text {cris }}^{\nabla}}$ (respectively $\operatorname{Mod}\left(\mathfrak{X}_{M}\right)_{\mathbb{B}_{\text {cris }}}$ and using 2.41 they define functors

$$
\operatorname{Mod}\left(\mathfrak{X}_{M}\right)_{\mathbb{B}_{\text {cris }}^{\nabla}} \longrightarrow \operatorname{Mod}-B_{\text {cris }}^{\nabla}\left(\bar{R}_{\mathcal{U}}\right)\left[\mathcal{G}_{\mathcal{U}, M}\right], \quad \operatorname{Mod}\left(\mathfrak{X}_{M}\right)_{\mathbb{B}_{\text {cris }}} \longrightarrow \operatorname{Mod}-B_{\text {cris }}\left(\bar{R}_{\mathcal{U}}\right)\left[\mathcal{G}_{\mathcal{U}, M}\right]
$$

to the categories of $B_{\text {cris }}^{\nabla}\left(\bar{R}_{\mathcal{U}}\right)$-modules (resp. $B_{\text {cris }}\left(\bar{R}_{\mathcal{U}}\right)$-modules) endowed with continuous action of $\mathcal{G}_{\mathcal{U}, M}$.

For later purposes we prove the following property of localizations of tensor products. In the next lemma we suppose that $M$ is a finite extension of $K$. Let $\mathcal{M}$ be a coherent sheaf of

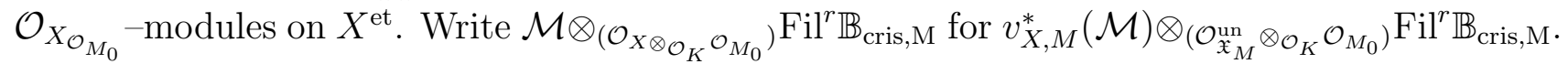

Lemma 3.2. Let $\mathbb{L}$ be a p-adic sheaf on $X_{M}^{\mathrm{et}}$. Fix $r \in \mathbb{Z} \cup\{-\infty\}$. Let $\mathcal{U} \in X^{\mathrm{et}}$ be a small affine. If $\mathcal{M}\left[p^{-1}\right](\mathcal{U})$ is a projective $R_{\mathcal{U}} \otimes_{\mathcal{O}_{K}} M_{0}$-module then

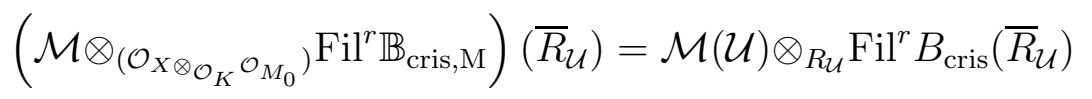

in $\operatorname{Mod}-B_{\text {cris }}\left(\bar{R}_{\mathcal{U}}\right)\left[\mathcal{G}_{\mathcal{U}, M}\right]$

Similarly let $V_{\mathcal{U}}(\mathbb{L})$ be the $\mathcal{G}_{\mathcal{U}, M}$-representation associated to $\mathbb{L}$. Then

$$
\left(\mathbb{L} \otimes_{\mathbb{Z}_{p}} \mathrm{Fil}^{r} \mathbb{B}_{\text {cris }, \mathrm{M}}\right)\left(\bar{R}_{\mathcal{U}}\right)=V_{\mathcal{U}}(\mathbb{L}) \otimes_{\mathbb{Z}_{p}} \mathrm{Fil}^{r} B_{\text {cris }}\left(\bar{R}_{\mathcal{U}}\right)
$$

in $\operatorname{Mod}-B_{\text {cris }}\left(\bar{R}_{\mathcal{U}}\right)\left[\mathcal{G}_{\mathcal{U}, M}\right]$ 
Proof. We prove the first statement. We assume that $X=\mathcal{U}$. Since $\mathcal{M}\left[p^{-1}\right]$ is projective and coherent it is a direct summand in a free $\mathcal{O}_{\mathcal{U}} \otimes_{\mathcal{O}_{K}} M_{0}$-module. We may then assume that $\mathcal{M}\left[p^{-1}\right]$ is free. The claim follows from 2.41, The second statement follows also from loc. cit.

Let $v_{M, *}^{\text {cont }}: \operatorname{Sh}\left(\mathfrak{X}_{M}\right)^{\mathbb{N}} \longrightarrow \operatorname{Sh}\left(X^{\text {et }}\right)$ be the functor $\left\{\mathcal{F}_{n}\right\}_{n} \mapsto \lim _{\infty \leftarrow n} v_{X, M, *}\left(\mathcal{F}_{n}\right)$. As explained in $\$ 2.8$ it induces a functor on the category $\operatorname{Ind}\left(\operatorname{Sh}\left(\mathfrak{X}_{M}\right)^{\mathbb{N}}\right)$ and hence a functor

$$
v_{M, *}: \operatorname{Mod}\left(\mathfrak{X}_{M}\right)_{\mathbb{B}_{\text {cris }}} \longrightarrow \operatorname{Sh}\left(X^{\mathrm{et}}\right) .
$$

Given a $p$-adic sheaf $\mathbb{L}$ on $X_{M}^{\text {et }}$ define

$$
\mathbb{D}_{\text {cris }, M}(\mathbb{L}):=v_{M, *}\left(\mathbb{L} \otimes_{\mathbb{Z}_{p}} \mathbb{B}_{\text {cris }, M}\right) .
$$

Recall that by abuse of notation we denoted $\mathbb{L}$ the continuous sheaf on $\mathfrak{X}_{M}$ given by $w_{X, M, *}(\mathbb{L})$. Then $\mathbb{D}_{\text {cris, } M}(\mathbb{L})$ is a sheaf of $\mathcal{O}_{X_{M_{0}}}$-modules in $\operatorname{Sh}\left(X^{\mathrm{et}}\right)$. Put

$$
\mathbb{D}_{\text {cris }}^{\text {geo }}(\mathbb{L}):=\mathbb{D}_{\text {cris }, \bar{K}}(\mathbb{L}), \quad \mathbb{D}_{\text {cris }}^{\text {ar }}(\mathbb{L}):=\mathbb{D}_{\text {cris }, \mathrm{M}}(\mathbb{L})
$$

whenever $M$ is a fixed finite extension of $K$.

Lemma 3.3. The sheaf $\mathbb{D}_{\text {cris }}^{\text {geo }}(\mathbb{L})$ is endowed with an action of $G_{M}$ and $\mathbb{D}_{\text {cris, },}(\mathbb{L})=\left(\mathbb{D}_{\text {cris }}^{\text {geo }}(\mathbb{L})\right)^{G_{M}}$. Proof. One has $v_{\bar{K}, *}^{\text {cont }}\left(\mathbb{L} \otimes_{\mathbb{Z}_{p}} \mathbb{A}_{\text {cris }, \bar{K}}(r)\right)=v_{M, *}^{\text {cont }}\left(\beta_{M, \bar{K}, *}^{\mathbb{N}}\left(\mathbb{L} \otimes_{\mathbb{Z}_{p}} \mathbb{A}_{\text {cris }, \bar{K}}(r)\right)\right)$ since $v_{\bar{K}, *}=v_{M, *} \circ$ $\beta_{M, \bar{K}, *}$. Due to corollary 2.32 we have $\beta_{M, \bar{K}}^{*}\left(\mathbb{A}_{\text {cris }, \mathrm{M}}\right) \cong \mathbb{A}_{\text {cris }, \overline{\mathrm{K}}}$ so that $v_{M, *}^{\text {cont }}\left(\beta_{M, \bar{K}, *}^{\mathbb{N}}\left(\mathbb{L} \otimes_{\mathbb{Z}_{p}} \mathbb{A}_{\text {cris }, \overline{\mathrm{K}}}(r)\right)\right)$ coincides with $v_{M, *}^{\operatorname{cont}}\left(\beta_{M, \bar{K}, *}^{\mathbb{N}} \circ \beta_{M, \bar{K}}^{\mathbb{N}, *}\left(\mathbb{L} \otimes_{\mathbb{Z}_{p}} \mathbb{A}_{\text {cris, }, \mathrm{M}}(r)\right)\right)$. It then follows from lemma 2.14(ii) that the module $v \overline{K, *}$ cont $\left(\mathbb{L} \otimes_{\mathbb{Z}_{p}} \mathbb{A}_{\text {cris }, \bar{K}}(r)\right)$ is endowed with an action of $G_{M}$ and that

$$
v_{M, *}^{\text {cont }}\left(\mathbb{L} \otimes_{\mathbb{Z}_{p}} \mathbb{A}_{\text {cris }, \mathrm{M}}(r)\right)=v \frac{\text { cont }}{K, *}\left(\mathbb{L} \otimes_{\mathbb{Z}_{p}} \mathbb{A}_{\text {cris }, \bar{K}}(r)\right)^{G_{M}} .
$$

Passing to direct limits on $r \in \mathbb{Z}$ the lemma follows.

Let $\mathcal{U}$ be a small affine of $X^{\text {et }}$. Then $\mathbb{L}_{n}\left(\bar{R}_{\mathcal{U}}\right)$ is a free $\mathbb{Z} / p^{n} \mathbb{Z}$-module by assumption and thus the natural map $\mathbb{L}_{n}\left(\bar{R}_{\mathcal{U}}\right) \otimes_{\mathbb{Z}_{p}} \mathbb{A}_{\text {cris,n,M}}^{\prime}\left(\bar{R}_{\mathcal{U}}\right) \longrightarrow\left(\mathbb{L}_{n} \otimes_{\mathbb{Z}_{p}} \mathbb{A}_{\text {cris,n,M }}^{\prime}\right)\left(\bar{R}_{\mathcal{U}}\right)$ is an isomorphism. Then $v_{M, *}\left(\mathbb{L}_{n} \otimes_{\mathbb{Z}_{p}} \mathbb{A}_{\text {cris,n,M }}^{\prime}\right)(\mathcal{U})=\left(\mathbb{L}_{n} \otimes_{\mathbb{Z}_{p}} \mathbb{A}_{\text {cris }, \mathrm{n}}\right)\left(\bar{R}_{\mathcal{U}}\right)^{\mathcal{G}_{\mathcal{U}, M}}$. Then the map $\mathbb{L}\left(\bar{R}_{\mathcal{U}}\right) \otimes_{\mathbb{Z}_{p}} A_{\text {cris }}\left(\bar{R}_{\mathcal{U}}\right) t^{r} \longrightarrow$ $\lim _{\infty \leftarrow n} \mathbb{L}_{n}\left(\bar{R}_{\mathcal{U}}\right) \otimes_{\mathbb{Z}_{p}} \mathbb{A}_{\text {cris,n }}^{\prime}\left(\bar{R}_{\mathcal{U}}\right) t^{r}$ is an isomorphism for every $r \in \mathbb{Z}$ since $A_{\text {cris }}\left(\bar{R}_{\mathcal{U}}\right)$ is $p$-adically complete and separated and thanks to proposition 2.34. Following [Bri] define

$$
D_{\text {cris, }, \mathrm{M}}\left(V_{\mathcal{U}}(\mathbb{L})\right):=\left(V_{\mathcal{U}}(\mathbb{L}) \otimes_{\mathbb{Z}_{p}} B_{\text {cris }}\left(\bar{R}_{\mathcal{U}}\right)\right)^{\mathcal{G}_{\mathcal{U}, M}} .
$$

It then follows that

$$
\mathbb{D}_{\text {cris, }, \mathrm{M}}(\mathbb{L})(\mathcal{U}) \stackrel{\sim}{\longrightarrow} D_{\text {cris }, \mathrm{M}}\left(V_{\mathcal{U}}(\mathbb{L})\right)
$$


as $R_{\mathcal{U}} \otimes_{\mathcal{O}_{K}} M_{0}$-modules and since $\mathcal{G}_{\mathcal{U}, \bar{K}}$ acts trivially on $t$ we get

$$
\left(V_{\mathcal{U}}(\mathbb{L}) \otimes_{\mathbb{Z}_{p}} B_{\text {cris }}\left(\bar{R}_{\mathcal{U}}\right)\right)^{\mathcal{G}_{\mathcal{U}, \bar{K}}} \stackrel{\sim}{\longrightarrow} \mathbb{D}_{\text {cris }}^{\text {geo }}(\mathbb{L})(\mathcal{U})
$$

as $R_{\mathcal{U}} \widehat{\otimes}_{\mathcal{O}_{K}} B_{\text {cris }}$-modules. Let $M$ be a finite extension of $K$. It follows that $\mathbb{D}_{\text {cris,M }}$ and $\mathbb{D}_{\text {cris }}^{\text {geo }}$ define functors

$$
\mathbb{D}_{\text {cris }}^{\text {ar }}: \operatorname{Sh}\left(X_{M}^{\mathrm{et}}\right)_{\mathbb{Q}_{p}} \longrightarrow \operatorname{Mod}_{\mathcal{O}_{X} \otimes \mathcal{O}_{K} M_{0}}
$$

and

$$
\mathbb{D}_{\text {cris }}^{\text {geo }}: \operatorname{Sh}\left(X_{M}^{\mathrm{et}}\right)_{\mathbb{Q}_{p}} \longrightarrow \operatorname{Mod}\left(\mathcal{O}_{X} \otimes_{\mathcal{O}_{K}} B_{\text {cris }}\right) ;
$$

here $\mathcal{O}_{X} \otimes_{\mathcal{O}_{K}} B_{\text {cris }}$ stands for $\mathcal{O}_{X} \widehat{\otimes}_{\mathcal{O}_{K}} A_{\text {cris }}\left[t^{-1}\right]$ where $\mathcal{O}_{X} \widehat{\otimes}_{\mathcal{O}_{K}} A_{\text {cris }}$ is the sheaf on $X^{\text {et }}$ defined by $\lim _{\infty \leftarrow n}\left(\left(\mathcal{O}_{X} / p^{n} \mathcal{O}_{X}\right) \otimes_{\mathcal{O}_{K}} A_{\text {cris }}\right)$. Furthermore we have.

Lemma 3.4. Let $M$ be a finite extension of $K$. The $R_{\mathcal{U}} \otimes_{\mathcal{O}_{K}} M_{0}-$ module $\mathbb{D}_{\text {cris }}^{\text {ar }}(\mathbb{L})(\mathcal{U})$ is projective, of finite type and of rank less or equal to the rank of $\mathbb{L}$.

Proof. It follows from [Bri, Prop. 8.3.1] and the identification $D_{\text {cris }}\left(V_{\mathcal{U}}(\mathbb{L})\right)=\mathbb{D}_{\text {cris }}^{\text {ar }}(\mathbb{L})(\mathcal{U})$.

Crystalline étale sheaves. Let $K \subseteq M(\subset \bar{K})$ be a finite extension. Following [O, Def. 1.1] we denote by $\operatorname{Coh}\left(\mathcal{O}_{X} \otimes_{\mathcal{O}_{K}} M_{0}\right)$ to be the full subcategory of sheaves of $\mathcal{O}_{X} \otimes_{\mathcal{O}_{K}} M_{0}$-modules isomorphic to $F \otimes_{\mathcal{O}_{K}} K$ for some coherent sheaf $F$ of $\mathcal{O}_{X} \otimes_{\mathcal{O}_{K}} \mathcal{O}_{M_{0}}$-modules on $X$. A $\mathbb{Q}_{p}$-adic sheaf $\mathbb{L}=\left\{\mathbb{L}_{n}\right\}_{n}$ on $X_{M}^{\mathrm{et}}$ is called crystalline if

i. $\mathbb{D}_{\text {cris }}^{\text {ar }}(\mathbb{L})$ is in $\operatorname{Coh}\left(\mathcal{O}_{X} \otimes_{\mathcal{O}_{K}} M_{0}\right)$;

ii. the natural map $\alpha_{\text {cris }, \mathbb{L}}: \mathbb{D}_{\text {cris }}^{\text {ar }}(\mathbb{L}) \otimes_{\left(\mathcal{O}_{X} \otimes_{\mathcal{O}_{K}} \mathcal{O}_{M_{0}}\right)} \mathbb{B}_{\text {cris,M }} \longrightarrow \mathbb{L} \otimes_{\mathbb{Z}_{p}} \mathbb{B}_{\text {cris,M }}$ is an isomorphism in $\operatorname{Mod}\left(\mathfrak{X}_{M}\right) \mathbb{B}_{\text {cris }}$.

Denote by $\operatorname{Sh}\left(X_{M}^{\text {et }}\right)_{\mathbb{Q}_{p}}^{\text {cris }}$ the full subcategory of $\operatorname{Sh}\left(X_{M}^{\text {et }}\right)_{\mathbb{Q}_{p}}$ consisting of crystalline sheaves.

Convention: For any coherent $\mathcal{O}_{X} \otimes_{\mathcal{O}_{K}} \mathcal{O}_{M_{0}}$-module $D$ we write

$$
D \otimes_{\left(\mathcal{O}_{X} \otimes_{\mathcal{O}_{K}} \mathcal{O}_{M_{0}}\right)} \mathbb{B}_{\text {cris, }, \mathrm{M}}:=v_{X, M}^{*}(D) \otimes_{\left(\mathcal{O}_{\mathfrak{X}_{M}}^{\text {un }} \otimes_{\mathcal{O}_{K}} \mathcal{O}_{M_{0}}\right)} \mathbb{B}_{\text {cris }, \mathrm{M}}
$$

Remark 3.5. To make sense of (ii) note that by adjunction we have a morphism

$$
f_{m}(\mathbb{L}): v_{X, M}^{*}\left(v_{M, *}\left(\mathbb{L} \otimes_{\mathbb{Z}_{p}} \mathbb{A}_{\text {cris }, \mathrm{M}}(m)\right)\right) \otimes_{\left(\mathcal{O}_{\mathfrak{X}_{M}}^{\text {un }} \otimes_{\mathcal{O}_{K}} \mathcal{O}_{M_{0}}\right)} \mathbb{A}_{\text {cris }, \mathrm{M}} \longrightarrow \mathbb{L} \otimes_{\mathbb{Z}_{p}} \mathbb{A}_{\text {cris }, \mathrm{M}}(m) .
$$

Recall that $\mathcal{O}_{\mathfrak{X}_{M}}^{\text {un }} \subset \mathcal{O}_{\mathfrak{X}_{M}}$ is identified with $v_{X, M}^{*}\left(\mathcal{O}_{X}\right)$ by lemma 2.13. Using proposition 3.6 we know that for $m \leq N$ large the $\mathcal{O}_{X} \otimes_{\mathcal{O}_{K}} \mathcal{O}_{M_{0}}$-module $D(m):=v_{M, *}\left(\mathbb{L} \otimes_{\mathbb{Z}_{p}} \mathbb{A}_{\text {cris,M }}(m)\right)$ is coherent and its image in $\operatorname{Coh}\left(\mathcal{O}_{X} \otimes_{\mathcal{O}_{K}} M_{0}\right)$ is $\mathbb{D}_{\text {cris }}^{\text {ar }}(\mathbb{L})$. Then $\alpha_{\text {cris }, \mathbb{L}}$ is the map in $\operatorname{Mod}\left(\mathfrak{X}_{M}\right)_{B_{\text {cris }}}$ induced by the $f_{m}(\mathbb{L})$ for $m \leq N$. Due to proposition 3.6 and since $X$ is noetherian

$$
v_{X, M}^{*}\left(v_{M, *}\left(\mathbb{L} \otimes_{\mathbb{Z}_{p}} \mathbb{A}_{\text {cris, }, M}(m)\right)\right) \otimes_{\left(\mathcal{O}_{\mathfrak{X}_{M}}^{\text {un }} \otimes_{\mathcal{O}_{K}} \mathcal{O}_{M_{0}}\right)} \mathbb{A}_{\text {cris }, M}
$$

is also isomorphic as inductive system to $D \otimes_{\mathcal{O}_{X} \otimes_{\mathcal{O}_{K}} \mathcal{O}_{M_{0}}} \mathbb{B}_{\text {cris,M }}$ for any coherent $\mathcal{O}_{X} \otimes_{\mathcal{O}_{K}} \mathcal{O}_{M_{0}}{ }^{-}$ module $D$ such that $D \otimes_{\mathcal{O}_{M_{0}}} M_{0} \cong \mathbb{D}_{\text {cris }}^{\text {ar }}(\mathbb{L})$ as $\mathcal{O}_{X} \otimes_{\mathcal{O}_{K}} M_{0}$-modules. 
Proposition 3.6. Let $\mathbb{L}$ be a p-adic sheaf. If $\mathbb{D}_{\text {cris }}^{\text {ar }}(\mathbb{L})$ is in $\operatorname{Coh}\left(\mathcal{O}_{X} \otimes_{\mathcal{O}_{K}} M_{0}\right)$ there exists a negative integer $N$ such that for every $m \leq N$ the natural morphism

$$
\mu_{m}: v_{M, *}\left(\mathbb{L} \otimes_{\mathbb{Z}_{p}} \mathbb{A}_{\text {cris }, M}(m)\right) \longrightarrow \mathbb{D}_{\text {cris }}^{\text {ar }}(\mathbb{L})
$$

is injective and is an isomorphism after inverting $p$ as $\mathcal{O}_{X} \otimes_{\mathcal{O}_{K}} M_{0}$-modules. For any such $m \leq N$ and every small affine $\mathcal{U} \in X^{\text {et }}$ the $R_{\mathcal{U}} \otimes_{\mathcal{O}_{K}} \mathcal{O}_{M_{0}}$-module $v_{M, *}^{\text {cont }}\left(\mathbb{L}_{\mathbb{Z}_{p}} \mathbb{A}_{\text {cris,M }}(m)\right)(\mathcal{U})$ is finitely generated and p-torsion free.

Proof. Since $X$ is noetherian, $K \subset M$ is a finite extension and $\mathbb{D}_{\text {cris }}^{\text {ar }}(\mathbb{L})$ is in $\operatorname{Coh}\left(\mathcal{O}_{X} \otimes_{\mathcal{O}_{K}} M_{0}\right)$ there exists $N \in \mathbb{N}$ such that $\mu_{m}$ is surjective after inverting $p$ for every $m \geq N$. Since the natural maps $\mathbb{L} \otimes_{\mathbb{Z}_{p}} \mathbb{A}_{\text {cris, }, \mathrm{M}}(r) \rightarrow \mathbb{L} \otimes_{\mathbb{Z}_{p}} \mathbb{A}_{\text {cris,M }}(s)$ are injective and $v_{M, *}^{\text {cont }}$ is left exact, $\mu_{m}$ is also injective. This proves the first statement.

Since $\mathbb{D}_{\text {cris }}^{\text {ar }}(\mathbb{L})(\mathcal{U})$ is a projective and finitely generated $R_{\mathcal{U}} \otimes_{\mathcal{O}_{K}} M_{0}$-module it is a direct summand in a finite and free $R_{\mathcal{U}} \otimes_{\mathcal{O}_{K}} M_{0}$-module $T_{M_{0}}$. Let $T$ be a free $R_{\mathcal{U}} \otimes_{\mathcal{O}_{K}} \mathcal{O}_{M_{0}}$-submodule of $T_{M_{0}}$ such that $T\left[p^{-1}\right]=T_{M_{0}}$. Let $n \in \mathbb{N}$ be large enough so that the image of $V_{\mathcal{U}}(\mathbb{L})$ in $\mathbb{D}_{\text {cris }}^{\text {ar }}(\mathbb{L})(\mathcal{U}) \otimes_{M_{0}} B_{\text {cris }}\left(\bar{R}_{\mathcal{U}}\right) \subset T_{M_{0}} \otimes_{M_{0}} B_{\text {cris }}\left(\bar{R}_{\mathcal{U}}\right)$ is contained in $T \cdot \frac{1}{p^{n}} \otimes_{R_{\mathcal{U}} \otimes_{\mathcal{O}_{K}} \mathcal{O}_{M_{0}}} A_{\text {cris }}\left(\bar{R}_{\mathcal{U}}\right)$. Then $v_{M, *}\left(\mathbb{L} \otimes_{\mathbb{Z}_{p}} \mathbb{A}_{\text {cris, }, M}(m)\right)(\mathcal{U})$ is $\left(V_{\mathcal{U}}(\mathbb{L}) \otimes_{\mathbb{Z}_{p}} A_{\text {cris }}\left(\bar{R}_{\mathcal{U}}\right) t^{m}\right)^{\mathcal{G}, M}$ and this is contained in the submodule $\left(T \cdot \frac{1}{p^{n}} \otimes_{R_{\mathcal{U}} \otimes_{\mathcal{O}}} A_{\text {cris }}\left(\bar{R}_{\mathcal{U}}\right) t^{m}\right)^{\mathcal{G}_{\mathcal{U}, M}}$.

Put $R^{\prime}:=\left(A_{\text {cris }}\left(\bar{R}_{\mathcal{U}}\right) t^{m}\right)^{\mathcal{G}_{\mathcal{U}, M}}$. It is $p$-adically complete and separated, it contains $R_{\mathcal{U}} \otimes_{\mathcal{O}_{K}} \mathcal{O}_{M_{0}}$ and it is contained in $\left(B_{\text {cris }}\left(\bar{R}_{\mathcal{U}}\right) t^{m}\right)^{\mathcal{G}_{\mathcal{U}, M}}=R_{\mathcal{U}} \otimes_{\mathcal{O}_{K}} \mathcal{O}_{M_{0}}\left[p^{-1}\right]$ by [Bri, Prop. 6.2.9]. We claim that this implies that there exists $n \in \mathbb{N}$ such that $p^{n} R^{\prime}$ is contained in $R_{\mathcal{U}} \otimes_{\mathcal{O}_{K}} \mathcal{O}_{M_{0}}$. If $R_{\mathcal{U}}$ were a complete dvr, the above conditions would imply the claim. In the general case replacing $R$ with the localization at a prime ideal $\mathcal{P}$ over $p$ and $\bar{R}_{\mathcal{U}}$ with $\bar{R}_{\mathcal{U}, \mathcal{P}}$ we deduce that there exists $n_{\mathcal{P}} \in \mathbb{N}$ such that $p^{n_{\mathcal{P}}} R^{\prime} \subset \widehat{R}_{\mathcal{U}, \mathcal{P}} \otimes_{\mathcal{O}_{K}} \mathcal{O}_{M_{0}}$. Taking $n$ to be the maximum of all the $n_{\mathcal{P}}$ 's we deduce that $R^{\prime} \subset R_{\mathcal{U}} \otimes_{\mathcal{O}_{K}} \mathcal{O}_{M_{0}}\left[p^{-1}\right]$ and also $p^{n} R^{\prime} \subset \widehat{R}_{\mathcal{U}, \mathcal{P}} \otimes_{\mathcal{O}_{K}} \mathcal{O}_{M_{0}}$. Since $R_{\mathcal{U}} \otimes_{\mathcal{O}_{K}} \mathcal{O}_{M_{0}}$ is normal we deduce the claim. Since $T$ is free $R_{\mathcal{U}} \otimes_{\mathcal{O}_{K}} \mathcal{O}_{M_{0}}$-module, we conclude that $v_{M, *}\left(\mathbb{L}_{\mathbb{Z}_{p}} \mathbb{A}_{\text {cris,M }}(m)\right)(\mathcal{U})$ is contained in $T \otimes_{\mathcal{O}_{K}} \mathcal{O}_{M_{0}} \cdot \frac{1}{p^{n}}$. In particular it is a finitely generated $R_{\mathcal{U}} \otimes_{\mathcal{O}_{K}} \mathcal{O}_{M_{0}}$-module as desired.

Let $\mathbb{L}$ be a $p$-adic sheaf on $X_{M}^{\text {et }}$. Following [Bri] for every small affine $\mathcal{U}$ of $X^{\text {et }}$ we say that $V_{\mathcal{U}}(\mathbb{L})$ is crystalline if the map $D_{\text {cris }}\left(V_{\mathcal{U}}(\mathbb{L})\right) \otimes_{R_{\mathcal{U}}} B_{\text {cris }}\left(\bar{R}_{\mathcal{U}}\right) \longrightarrow V_{\mathcal{U}}\left(\mathbb{L}_{)} \otimes_{\mathbb{Z}_{p}} B_{\text {cris }}\left(\bar{R}_{\mathcal{U}}\right)\right.$ is an isomorphism. Then we have.

Proposition 3.7. The following are equivalent:

1) $\mathbb{L}$ is crystalline;

2) for every small affine object $\mathcal{U}$ of $X^{\mathrm{et}}$ the representation $V_{\mathcal{U}}(\mathbb{L})$ is crystalline;

3) there is a covering $\left\{\mathcal{U}_{i}\right\}_{i}$ of $X^{\text {et }}$ by small affine objects such that $V_{\mathcal{U}_{i}}(\mathbb{L})$ is crystalline for every $i$;

Proof. (1) $\Longrightarrow(2)$ Due to 3.2 we have $\left(\mathbb{L} \otimes_{\mathbb{Z}_{p}} \mathbb{B}_{\text {cris, }, \mathrm{M}}\right)\left(\bar{R}_{\mathcal{U}}\right)=V_{\mathcal{U}}(\mathbb{L}) \otimes_{\mathbb{Z}_{p}} B_{\text {cris }}\left(\bar{R}_{\mathcal{U}}\right)$. Note that $\mathbb{D}_{\text {cris }}^{\text {ar }}(\mathbb{L})(\mathcal{U})$ is a projective $R_{\mathcal{U}} \otimes_{\mathcal{O}_{K}} M_{0}$-module by 3.4 i.e. it is a direct summand in a free module. As a consequence of remark 3.5 and lemma 3.2 it follows that the localization of 
$\mathbb{D}_{\text {cris }}^{\text {ar }}(\mathbb{L}) \otimes_{\left(\mathcal{O}_{X} \otimes_{\mathcal{O}_{K}} \mathcal{O}_{M_{0}}\right)} \mathbb{B}_{\text {cris, M }}$ is isomorphic to $\mathbb{D}_{\text {cris }}^{\text {ar }}(\mathbb{L})(\mathcal{U}) \otimes_{\left(R_{\mathcal{U}} \otimes_{\mathcal{O}_{K}} \mathcal{O}_{M_{0}}\right)} B_{\text {cris }}\left(\bar{R}_{\mathcal{U}}\right)$. The implication follows applying the localization functor to the isomorphism $\mathbb{D}_{\text {cris }}^{\text {ar }}(\mathbb{L}) \otimes_{\left(\mathcal{O}_{X} \otimes_{\mathcal{O}_{K}} \mathcal{O}_{M_{0}}\right)} \mathbb{B}_{\text {cris, M }} \longrightarrow$ $\mathbb{L} \otimes_{\mathbb{Z}_{p}} \mathbb{B}_{\text {cris,M }}$

$(2) \Longrightarrow(3)$ is clear.

$(3) \Longrightarrow(1)$ For a small affine open $\mathcal{U}$ of $X^{\text {et }}$ and for a negative integer $r$ let $g_{\mathcal{U}, r}$ be the natural map

$$
g_{\mathcal{U}, r}:\left(V_{\mathcal{U}}(\mathbb{L}) \otimes_{\mathbb{Z}_{p}} A_{\text {cris }}\left(\bar{R}_{\mathcal{U}}\right) t^{r}\right)^{\mathcal{G}_{\mathcal{U}, M}} \otimes_{R_{\mathcal{U}}} A_{\text {cris }}\left(\bar{R}_{\mathcal{U}}\right) \longrightarrow V_{\mathcal{U}}(\mathbb{L}) \otimes_{\mathbb{Z}_{p}} A_{\text {cris }}\left(\bar{R}_{\mathcal{U}}\right) t^{r}
$$

It is injective by [Bri, Prop. 8.2.6]. In particular $V_{\mathcal{U}}(\mathbb{L})$ is crystalline if and only if $V_{\mathcal{U}}(\mathbb{L})$ is in the image of $g_{\mathcal{U}, r}$ for some $r<0$. We deduce that if $V_{\mathcal{U}}(\mathbb{L})$ is crystalline then $V_{\mathcal{V}}(\mathbb{L})$ is crystalline for every open affine $\mathcal{V} \rightarrow \mathcal{U}$.

Fix a small affine $\mathcal{U}$ which factors through one of the $\mathcal{U}_{i}$ 's. In particular $V_{\mathcal{U}}(\mathbb{L})$ is crystalline. Assume that $V_{\mathcal{U}}(\mathbb{L})$ is in the image of $g_{\mathcal{U}, r}$. Let $\mathcal{V} \rightarrow \mathcal{U}$ be an étale morphism with $\mathcal{V}$ affine. Let $D^{\prime} \subset D_{\text {cris }}\left(V_{\mathcal{V}}(\mathbb{L})\right)$ be the image of $D_{\text {cris }}\left(V_{\mathcal{U}}(\mathbb{L})\right) \rightarrow D_{\text {cris }}\left(V_{\mathcal{V}}(\mathbb{L})\right)$. Since $V_{\mathcal{U}}(\mathbb{L})=V_{\mathcal{V}}(\mathbb{L})$, then $V_{\mathcal{V}}(\mathbb{L})$ is in the image of $g_{\mathcal{V}, r}$ and also $V_{\mathcal{V}}(\mathbb{L})$ is crystalline. The extension $R_{\mathcal{V}} \otimes_{\mathcal{O}_{K}} M_{0} \rightarrow$ $B_{\text {cris }}\left(\bar{R}_{\mathcal{V}}\right)$ is faithfully flat by [Bri, Thm. 6.3.8] so that the maps

$$
D^{\prime} \otimes_{R_{\mathcal{U}}} R_{\mathcal{V}} \otimes_{\left(R_{\mathcal{V}} \otimes_{\mathcal{O}_{K}} M_{0}\right)} B_{\text {cris }}\left(\bar{R}_{\mathcal{V}}\right) \rightarrow D_{\text {cris }}\left(V_{\mathcal{V}}(\mathbb{L})\right) \otimes_{R_{\mathcal{V}}} B_{\text {cris }}\left(\bar{R}_{\mathcal{V}}\right) \rightarrow V_{\mathcal{V}}(\mathbb{L}) \otimes_{\mathbb{Z}_{p}} B_{\text {cris }}\left(\bar{R}_{\mathcal{V}}\right)
$$

are all injective and the composite is surjective. Thus $D^{\prime} \otimes_{R_{\mathcal{U}}} R_{\mathcal{V}}=D_{\text {cris }}\left(V_{\mathcal{V}}(\mathbb{L})\right)$. This proves that $\mathcal{V} \mapsto D_{\text {cris }}\left(V_{\mathcal{V}}(\mathbb{L})\right)$ is a coherent $\mathcal{O}_{X_{M_{0}}}-$ module. Since $D_{\text {cris }}\left(V_{\mathcal{V}}(\mathbb{L})\right) \cong \mathbb{D}_{\text {cris,M }}(\mathbb{L})(\mathcal{V})$ it follows that $\left.\mathbb{D}_{\text {cris,M }}(\mathbb{L})\right|_{\mathcal{U}}$ is a coherent $\mathcal{O}_{\mathcal{U}_{M_{0}}}$-module as well. We deduce from [O, Prop. 1.2] that $\mathbb{D}_{\text {cris,M }}(\mathbb{L})$ lies in $\operatorname{Coh}\left(\mathcal{O}_{X} \otimes_{\mathcal{O}_{K}} M_{0}\right)$ i. e., condition (i) in the definition of a crystalline étale sheaf holds.

We can be more explicit. Take $N<0$ as in 3.6. Put $D:=\left(V_{\mathcal{U}}\left(\mathbb{L}_{)} \otimes_{\mathbb{Z}_{p}} A_{\text {cris }}\left(\bar{R}_{\mathcal{U}}\right) t^{N}\right)^{\mathcal{G}_{\mathcal{U}, M}}\right.$ and $V:=V_{\mathcal{U}}(\mathbb{L})$. From the proof of 3.6 it follows that $D$ is a finitely generated $R_{\mathcal{U}} \otimes_{\mathcal{O}_{K}} \mathcal{O}_{M_{0}}$-module and by construction $D^{\prime} \otimes_{\mathcal{O}_{K}} K=\mathbb{D}_{\text {cris, } M}(\mathbb{L})(\mathcal{U})$. Since $X$ is a noetherian topological space, this implies that $\mathbb{D}_{\text {cris, } M}(\mathbb{L})$ lies in $\operatorname{Coh}\left(\mathcal{O}_{X} \otimes_{\mathcal{O}_{K}} M_{0}\right)$. Consider the commutative diagram

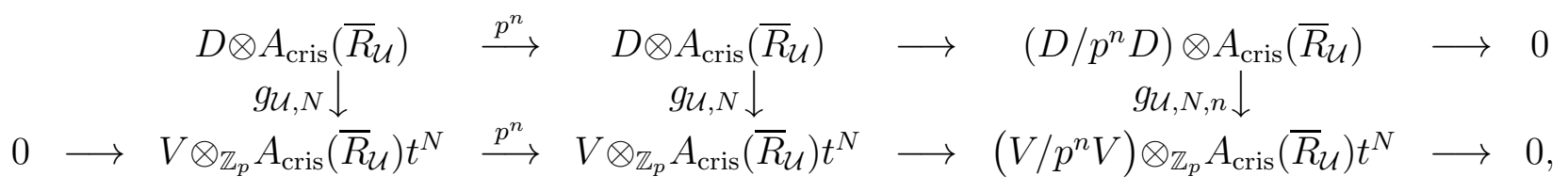

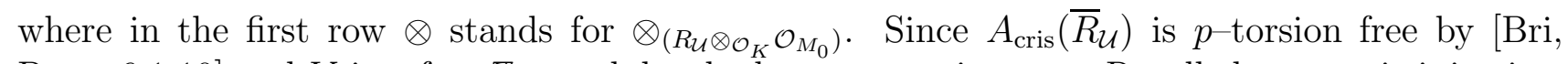
Prop. 6.1.10] and $V$ is a free $\mathbb{Z}_{p}$-module, the bottom row is exact. Recall that $g_{\mathcal{U}, N}$ is injective. Since (3) holds there exists $N$ such that $V$ is in the image of $g_{\mathcal{U}, N}$. Then the cokernel of $g_{\mathcal{U}, N}$ is annihilated by $t^{-N}$. Since for every open affine $\mathcal{V} \in \mathfrak{U}_{K}$ we have $V_{\mathcal{U}}(\mathbb{L}) \cong V_{\mathcal{V}}(\mathbb{L})$ we deduce that also the cokernel of $g_{\mathcal{V}, N}$ is annihilated by $t^{-N}$. Thus the kernel and cokernel of $g_{\mathcal{V}, N, n}$ are annihilated by $t^{-N}$.

Write $f_{N}$ for the system of morphisms

$$
f_{N, n}: v_{M, *}\left(\mathbb{L} \otimes_{\mathbb{Z}_{p}} \mathbb{A}_{\text {cris,n,M }}^{\prime}(m)\right) \otimes_{\left(\mathcal{O}_{X} \otimes_{\mathcal{O}_{K}} \mathcal{O}_{M_{0}}\right)} \mathbb{A}_{\text {cris,M }} \longrightarrow \mathbb{L} \otimes_{\mathbb{Z}_{p}} \mathbb{A}_{\text {cris }, \mathrm{n}, \mathrm{M}}(m)
$$


given by adjunction. For every $x \in \mathcal{U}$ the stalk $\mathbb{A}_{\text {cris,n,M,x }}^{\prime}$ at $x$ contains $\lim _{x \in \mathcal{V}} A_{\text {cris }}\left(\bar{R}_{\mathcal{V}}\right) / p^{n} A_{\text {cris }}\left(\bar{R}_{\mathcal{V}}\right)$ where the limit is taken over all affine opens $\mathcal{V}$ of $x$. The cokernel of $\lim _{x \in \mathcal{V}} A_{\text {cris }}\left(\bar{R}_{\mathcal{V}}\right) / p^{n} A_{\text {cris }}\left(\bar{R}_{\mathcal{U}}\right) \subset$ $\mathbb{A}_{\text {cris,n,M,x }}^{\prime}$ is annihilated by any element of $\mathbb{I}$ by 2.35 and hence also by $t$. Since $\mathbb{L}_{x}=V_{\mathcal{U}}(\mathbb{L})$ and $f_{N, n, x}$ is $\lim _{x \in \mathcal{V}} g_{\mathcal{V}, N, n}$ on $\lim _{x \in \mathcal{V}} D \otimes A_{\text {cris }}\left(\bar{R}_{\mathcal{V}}\right) / p^{n} A_{\text {cris }}\left(\bar{R}_{\mathcal{U}}\right)$, we conclude that kernel and cokernel of $f_{N, n, x}$ is annihilated by $t^{-N+1}$. Since $X$ is a noetherian space and taking a smaller $N$ if necessary, we may assume that kernel and cokernel of $f_{N, n, x}$ is annihilated by $t^{-N+1}$ for every $x \in X$. Thus the same applies to $f_{N, n}$ and (1) follows from 3.1 (3).

\subsection{The functors $\mathbb{D}_{\text {cris }}^{\text {ar }}$ and $\mathbb{V}_{\text {cris }}^{\text {ar }}$ on crystalline sheaves.}

Assume as before that $\mathcal{O}_{K}=\mathbb{W}(k)$ and let $K \subseteq M$ be a field extension. The goal of this section is to prove in 3.12 that $\mathbb{D}_{\text {cris }}^{\text {ar }}$ defines an exact, fully faithful functor, commuting with tensor products, duals and Tate twists, from the category of $\mathbb{Q}_{p}$-adic crystalline shaves on $X_{M}^{\mathrm{et}}$ to the category of admissible filtered convergent $F$-isocrystals on the special fiber of $X$ relatively to $M_{0}$. We also construct an inverse $\mathbb{V}_{\text {cris }}^{\text {ar }}$ on the essential image.

Given a $p$-adic sheaf $\mathbb{L}$ on $X_{M}^{\text {et }}$ and $r \in \mathbb{Z}$ we get a well defined subsheaf

$$
\mathrm{Fil}^{r} \mathbb{D}_{\text {cris }, \mathrm{M}}(\mathbb{L}):=v_{M, *}\left(\mathbb{L} \otimes_{\mathbb{Z}_{p}} \mathrm{Fil}^{r} \mathbb{B}_{\text {cris }, \mathrm{M}}\right) \subset \mathbb{D}_{\text {cris }, \mathrm{M}}(\mathbb{L}) \text {. }
$$

Put $\mathrm{Fil}^{r} \mathbb{D}_{\text {cris }}^{\text {geo }}(\mathbb{L}):=\mathrm{Fil}^{r} \mathbb{D}_{\text {cris }, \overline{\mathrm{K}}}(\mathbb{L})$ and if $K \subset M$ is a fixed finite extension, $\mathrm{Fil}^{r} \mathbb{D}_{\text {cris }}^{\text {ar }}(\mathbb{L}):=$ $\mathrm{Fil}^{r} \mathbb{D}_{\text {cris, }, \mathrm{M}}(\mathbb{L})$. It follows from 3.3 that $\mathrm{Fil}^{r} \mathbb{D}_{\text {cris, } \mathrm{M}}(\mathbb{L})=\left(\mathrm{Fil}^{r} \mathbb{D}_{\text {cris }}^{\text {geo }}(\mathbb{L})\right)^{G_{M}}$.

Since the connections $\nabla(r): \mathbb{A}_{\text {cris,M }}(r) \longrightarrow \mathbb{A}_{\text {cris,M }}(r) \otimes_{\mathcal{O}_{X}} \Omega_{X / \mathcal{O}_{K}}^{1}$ are compatible for varying $r$ they induce a connection

$$
\nabla_{\mathbb{L}}: \mathbb{D}_{\text {cris }, \mathrm{M}}(\mathbb{L}) \longrightarrow \mathbb{D}_{\text {cris }, \mathrm{M}}(\mathbb{L}) \otimes_{\mathcal{O}_{X}} \Omega_{X / \mathcal{O}_{K}}^{1}
$$

Given a small affine $\mathcal{U}$ of $X^{\text {et }}$, write $V_{\mathcal{U}}(\mathbb{L})$ for $p$-adic representation of $\mathcal{G}_{\mathcal{U}_{M}}$ defined by $\mathbb{L}\left(\bar{R}_{\mathcal{U}}\right)$ and put

$$
\operatorname{Fil}^{r} D_{\text {cris }}\left(V_{\mathcal{U}}(\mathbb{L})\right):=\left(V_{\mathcal{U}}\left(\mathbb{L}_{)} \otimes_{\mathbb{Z}_{p}} \operatorname{Fil}^{r} B_{\text {cris }}\left(\bar{R}_{\mathcal{U}}\right)\right)^{\mathcal{G}_{\mathcal{U}, M}}\right.
$$

Due to 2.36 we deduce that via the identification $D_{\text {cris }}\left(V_{\mathcal{U}}\left(\mathbb{L}_{)}\right) \cong \mathbb{D}_{\text {cris,M }}\left(\mathbb{L}_{)}\right)\left(\bar{R}_{\mathcal{U}}\right)\right.$ we have Fil $^{r} D_{\text {cris }}\left(V_{\mathcal{U}}(\mathbb{L})\right) \cong$ Fil $^{r} \mathbb{D}_{\text {cris,M }}(\mathbb{L})\left(\bar{R}_{\mathcal{U}}\right)$ for every $r \in \mathbb{Z}$. For every $r \in \mathbb{Z}$ define the filtrations

$$
\operatorname{Fil}^{r}\left(\mathbb{D}_{\text {cris }}^{\text {ar }}(\mathbb{L}) \otimes_{\left(\mathcal{O}_{X} \otimes_{\mathcal{O}_{K}} \mathcal{O}_{M_{0}}\right)} \mathbb{B}_{\text {cris,M }}\right):=\sum_{a+b=r} \operatorname{Fil}^{a} \mathbb{D}_{\text {cris }}^{\text {ar }}(\mathbb{L}) \otimes_{\left(\mathcal{O}_{X} \otimes_{\mathcal{O}_{K}} \mathcal{O}_{M_{0}}\right)} \text { Fil }^{b} \mathbb{B}_{\text {cris,M }}
$$

and $\mathrm{Fil}^{r}\left(\mathbb{L} \otimes_{\mathbb{Z}_{p}} \mathbb{B}_{\text {cris,M }}\right):=\mathbb{L} \otimes_{\mathbb{Z}_{p}} \mathrm{Fil}^{r} \mathbb{B}_{\text {cris,M }}$ by sub-objects in the category $\operatorname{Ind}\left(\operatorname{Sh}\left(\mathfrak{X}_{M}\right)^{\mathbb{N}}\right)$ of inductive systems of continuous sheaves. We denote by

$$
\left.\operatorname{Gr}^{r}\left(\mathbb{D}_{\text {cris }}^{\text {ar }}(\mathbb{L}) \otimes_{\left(\mathcal{O}_{X} \otimes \mathcal{O}_{K}\right.} \mathcal{O}_{M_{0}}\right) \mathbb{B}_{\text {cris, }, M}\right)
$$

and $\operatorname{Gr}^{r}\left(\mathbb{L} \otimes_{\mathbb{Z}_{p}} \mathbb{B}_{\text {cris,M }}\right)$ the $r$-th graded quotients of the two filtrations (which are objects in $\left.\operatorname{Ind}\left(\operatorname{Sh}\left(\mathfrak{X}_{M}\right)^{\mathbb{N}}\right)\right)$. 
We will assume now until the end of this section that $\mathbb{L}$ is a crystalline sheaf on $X_{M}^{\mathrm{et}}$. By construction the isomorphism

$$
\alpha_{\text {cris }, \mathbb{L}}: \mathbb{D}_{\text {cris }}^{\text {ar }}(\mathbb{L}) \otimes_{\left(\mathcal{O}_{X} \otimes_{\mathcal{O}_{K}} \mathcal{O}_{M_{0}}\right)} \mathbb{B}_{\text {cris }, \mathrm{M}} \cong \mathbb{L} \otimes_{\mathbb{Z}_{p}} \mathbb{B}_{\text {cris }, \mathrm{M}}
$$

has the property that $\alpha_{\text {cris }, \mathbb{L}}\left(\mathrm{Fil}^{r}\left(\mathbb{D}_{\text {cris }}^{\text {ar }}(\mathbb{L}) \otimes_{\left(\mathcal{O}_{X} \otimes_{\mathcal{O}_{K}} \mathcal{O}_{M_{0}}\right)} \mathbb{B}_{\text {cris,M }}\right)\right) \subset \mathrm{Fil}^{r}\left(\mathbb{L} \otimes_{\mathbb{Z}_{p}} \mathbb{B}_{\text {cris,M }}\right)$ and thus it induces a morphism $\operatorname{Gr}^{r} \alpha_{\text {cris, } \mathbb{L}}$ on $\mathrm{Gr}^{r}$.

Lemma 3.8. For every $r \in \mathbb{Z}$ the natural morphism

$$
f: \bigoplus_{a+b=r} \operatorname{Gr}^{a} \mathbb{D}_{\text {cris }}^{\text {ar }}(\mathbb{L}) \otimes_{\left(\mathcal{O}_{X} \otimes_{\mathcal{O}_{K}} \mathcal{O}_{M_{0}}\right)} \operatorname{Gr}^{b} \mathbb{B}_{\text {cris, }, \mathrm{M}} \longrightarrow \operatorname{Gr}^{r}\left(\mathbb{L} \otimes_{\mathbb{Z}_{p}} \mathbb{B}_{\text {cris, }, \mathrm{M}}\right)
$$

is an isomorphism. In particular $\operatorname{Gr}^{r} \alpha_{\text {cris, } \mathbb{L}}$ is an isomorphism.

Proof. The surjective morphisms

$$
\bigoplus_{a+b=s} \mathrm{Fil}^{a} \mathbb{D}_{\text {cris }}^{\text {ar }}(\mathbb{L}) \otimes_{\left(\mathcal{O}_{X} \otimes_{\mathcal{O}_{K}} \mathcal{O}_{M_{0}}\right)} \mathrm{Fil}^{b} \mathbb{B}_{\text {cris }, \mathrm{M}} \longrightarrow \mathrm{Fil}^{s}\left(\mathbb{D}_{\text {cris }}^{\text {ar }}(\mathbb{L}) \otimes_{\left(\mathcal{O}_{X} \otimes_{\mathcal{O}_{K}} \mathcal{O}_{M_{0}}\right)} \mathbb{B}_{\text {cris,M }}\right)
$$

for $s=r$ and $r+1$ induce a surjective morphism

$$
\bigoplus_{a+b=r} \operatorname{Gr}^{a} \mathbb{D}_{\text {cris }}^{\text {ar }}(\mathbb{L}) \otimes_{\left(\mathcal{O}_{X} \otimes_{\mathcal{O}_{K}} \mathcal{O}_{M_{0}}\right)} \operatorname{Gr}^{b} \mathbb{B}_{\text {cris, }, \mathrm{M}} \longrightarrow \operatorname{Gr}^{r}\left(\mathbb{D}_{\text {cris }}^{\text {ar }}(\mathbb{L}) \otimes_{\left(\mathcal{O}_{X} \otimes_{\mathcal{O}_{K}} \mathcal{O}_{M_{0}}\right)} \mathbb{B}_{\text {cris,M }}\right)
$$

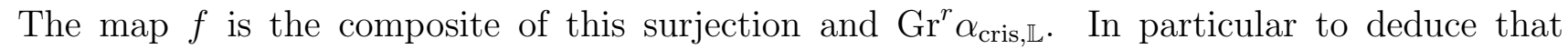
$\operatorname{Gr}^{r} \alpha_{\text {cris }, \mathbb{L}}$ is an isomorphism we are left to prove that $f$ is an isomorphism.

For every integer $N$ define $D(N):=v_{M, *}\left(\mathbb{L} \otimes_{\mathbb{Z}_{p}} \mathbb{A}_{\text {cris, }, \mathrm{M}}(N)\right)$ with the induced filtration. It follows from 3.6 that $\bigoplus_{a+b=r} \operatorname{Gr}^{a} \mathbb{D}_{\text {cris }}^{\text {ar }}(\mathbb{L}) \otimes_{\left(\mathcal{O}_{X} \otimes \mathcal{O}_{K} \mathcal{O}_{M_{0}}\right)} \operatorname{Gr}^{b} \mathbb{B}_{\text {cris,M }}$ is, for $N$ sufficiently small the inductive system of continuous sheaves $\bigoplus_{a+b=r} \operatorname{Gr}^{a} D(N) \otimes_{\left(\mathcal{O}_{X} \otimes_{\mathcal{O}_{K}} \mathcal{O}_{M_{0}}\right)} \mathrm{Gr}^{b} \mathbb{A}_{\text {cris,M }}(m)$ and $\operatorname{Gr}^{r}\left(\mathbb{L} \otimes_{\mathbb{Z}_{p}} \mathbb{B}_{\text {cris,M }}\right)$ is the inductive system of continuous sheaves $\mathbb{L} \otimes_{\mathbb{Z}_{p}} \operatorname{Gr}^{r}\left(\mathbb{A}_{\text {cris,M }}(m)\right)$. Furthermore $f$ is induced by the natural morphisms

$$
f_{m}: \bigoplus_{a+b=r} \operatorname{Gr}^{a} D(N) \otimes_{\left(\mathcal{O}_{X} \otimes_{\mathcal{O}_{K}} \mathcal{O}_{M_{0}}\right)} \operatorname{Gr}^{b} \mathbb{A}_{\text {cris }, \mathrm{M}}(m) \longrightarrow \mathbb{L} \otimes_{\mathbb{Z}_{p}} \operatorname{Gr}^{r}\left(\mathbb{A}_{\text {cris }, \mathrm{M}}(N+m)\right)
$$

To conclude it would be enough to show that there exists a negative integer $N$ and morphisms

$$
g_{m}: \mathbb{L} \otimes_{\mathbb{Z}_{p}} \operatorname{Gr}^{r}\left(\mathbb{A}_{\text {cris, } \mathrm{M}}(m)\right) \longrightarrow \bigoplus_{a+b=r} \operatorname{Gr}^{a} D(N) \otimes_{\left(\mathcal{O}_{X} \otimes_{\mathcal{O}_{K}} \mathcal{O}_{M_{0}}\right)} \operatorname{Gr}^{b} \mathbb{A}_{\text {cris }, \mathrm{M}}(m+N)
$$

such that $g_{N+m} \circ f_{m}$ and $f_{m+N} \circ g_{m}$ induce automorphisms on the two inductive systems.

Consider a small affine $\mathcal{U}$ of $X^{\text {et }}$. Let $V_{\mathcal{U}}(\mathbb{L})=\mathbb{L}\left(\bar{R}_{\mathcal{U}}\right)$ be the associated representation of $\mathcal{G}_{\mathcal{U}}$. It is crystalline in the sense of [Bri] thanks to 3.7 with $D_{\text {cris }}\left(V_{\mathcal{U}}(\mathbb{L})\right)=\mathbb{D}_{\text {cris }}^{\text {ar }}(\mathbb{L})(\mathcal{U})$. It follows from [Bri, Prop. 8.2.12] that it is de Rham with $D_{\mathrm{dR}}\left(V_{\mathcal{U}}(\mathbb{L})\right)=D_{\text {cris }}\left(V_{\mathcal{U}}(\mathbb{L})\right)$ and from [Bri, Prop. 8.3.2] that it is Hodge-Tate with $D_{\mathrm{HT}}\left(V_{\mathcal{U}}(\mathbb{L})\right)=\operatorname{Gr} D_{\mathrm{dR}}\left(V_{\mathcal{U}}(\mathbb{L})\right)=\operatorname{Gr} D_{\text {cris }}\left(V_{\mathcal{U}}(\mathbb{L})\right)$ i.e. we have an isomorphism $D_{\mathrm{HT}}\left(V_{\mathcal{U}}(\mathbb{L})\right) \otimes_{\left(R_{\mathcal{U}} \otimes_{\mathcal{O}_{K}} \mathcal{O}_{M_{0}}\right)} B_{\mathrm{HT}}\left(\bar{R}_{\mathcal{U}}\right) \cong V_{\mathcal{U}}(\mathbb{L}) \otimes_{\mathbb{Z}_{p}} B_{\mathrm{HT}}\left(\bar{R}_{\mathcal{U}}\right)$ as graded modules. 
Using the identifications $B_{\mathrm{HT}}\left(\bar{R}_{\mathcal{U}}\right)=\operatorname{Gr}\left(B_{\text {cris }}\left(\bar{R}_{\mathcal{U}}\right)\right)$ (see [Bri, Cor. 5.2.7]) and $\operatorname{Gr}\left(B_{\text {cris }}\left(\bar{R}_{\mathcal{U}}\right)\right)=$ $\lim _{\rightarrow, m} \operatorname{Gr}_{\text {cris, }, \mathrm{M}}(m)\left(\bar{R}_{\mathcal{U}}\right)$ (see 2.41) we obtain that in the following diagram the vertical arrows are isomorphisms:

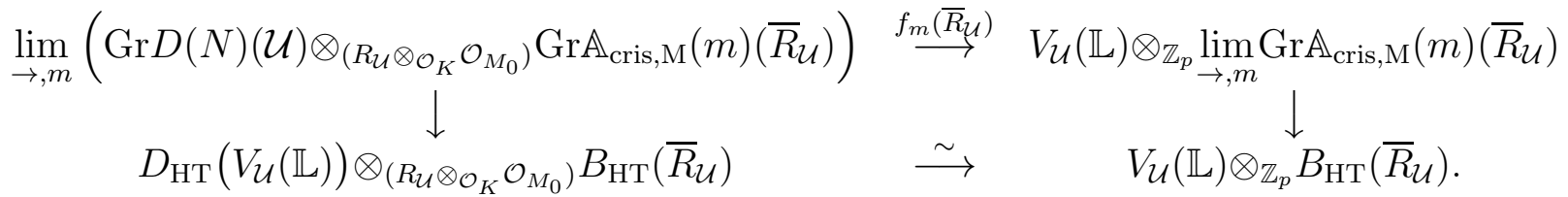

In particular since $V_{\mathcal{U}}(\mathbb{L})$ is a free $\mathbb{Z}_{p}$-module of finite rank there exists a negative integer $Q$ such that $V_{\mathcal{U}}(\mathbb{L})$ is contained in the image of $f_{Q}\left(\bar{R}_{\mathcal{U}}\right)$. Since $\operatorname{Gr} D(N)(\mathcal{U})\left[p^{-1}\right]=\operatorname{GrD}_{\text {cris }}^{\text {ar }}(\mathcal{U})=$ $D_{\mathrm{HT}}\left(V_{\mathcal{U}}(\mathbb{L})\right)$ and the latter is a projective $R_{\mathcal{U}} \otimes_{\mathcal{O}_{K}} M_{0}$-module by [Bri, Prop. 8.3.2], there exists $h \in \mathbb{N}$ and a free $R_{\mathcal{U}} \otimes_{\mathcal{O}_{K}} \mathcal{O}_{M_{0}}$-module $T$ and maps $a: \operatorname{Gr} D(N)(\mathcal{U}) \rightarrow T$ and $b: T \rightarrow \operatorname{Gr} D(N)(\mathcal{U})$ such that $b \circ a$ is multiplication by $p^{h}$.

We claim that $p^{h}$ annihilates the kernel of the natural map

$$
f: \operatorname{Gr} D(N)(\mathcal{U}) \otimes_{\left(R_{\mathcal{U}} \otimes_{\mathcal{O}_{K}} \mathcal{O}_{M_{0}}\right)} \operatorname{Gr} \mathbb{A}_{\text {cris, }, \mathrm{M}}(m)\left(\bar{R}_{\mathcal{U}}\right) \longrightarrow D_{\mathrm{HT}}\left(V_{\mathcal{U}}(\mathbb{L})\right) \otimes_{\left(R_{\mathcal{U}} \otimes_{\mathcal{O}_{K}} \mathcal{O}_{M_{0}}\right)} B_{\mathrm{HT}}\left(\bar{R}_{\mathcal{U}}\right)
$$

To see this let us first remark that as $T$ is a free $R_{\mathcal{U}} \otimes_{\mathcal{O}_{K}} \mathcal{O}_{M_{0}}$-module, the natural map

$$
T \otimes_{\left(R_{\mathcal{U}} \otimes_{\mathcal{O}_{K}} \mathcal{O}_{M_{0}}\right)} \operatorname{GrA}_{\text {cris, } \mathrm{M}}(m)\left(\bar{R}_{\mathcal{U}}\right) \longrightarrow T \otimes_{\left(R_{\mathcal{U}} \otimes_{\mathcal{O}_{K}} \mathcal{O}_{M_{0}}\right)} B_{\mathrm{HT}}\left(\bar{R}_{\mathcal{U}}\right)
$$

is injective. We have the following commutative diagram in which all the tensor products are over $R_{\mathcal{U}} \otimes_{\mathcal{O}_{K}} \mathcal{O}_{M_{0}}$ and we have denoted by $A_{M, m, \mathcal{U}}$ the ring $\operatorname{Gr} \mathbb{A}_{\text {cris, } \mathrm{M}}(m)\left(\bar{R}_{\mathcal{U}}\right)$.

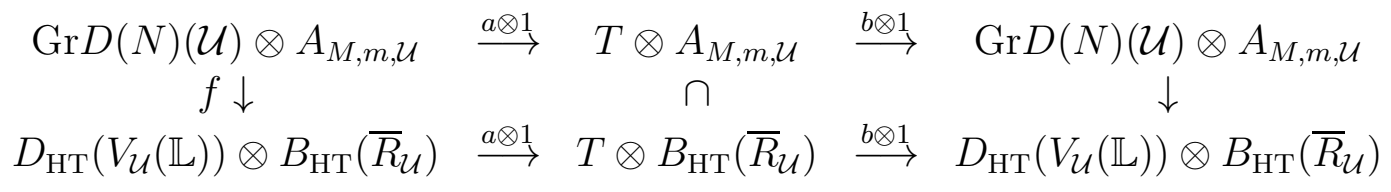

Let $x \in \operatorname{Ker}(f)$. Then $p^{h} x=(b \otimes 1)(a \otimes 1(x))=0$ which proves the claim.

Now we choose the pre-image of basis elements of $V_{\mathcal{U}}(\mathbb{L})$ in

$$
\left(\operatorname{Gr} D(N)(\mathcal{U}) \otimes_{\left(R_{\mathcal{U}} \otimes_{\mathcal{O}_{K}} \mathcal{O}_{M_{0}}\right)} \operatorname{Gr} \mathbb{A}_{\text {cris,M }}(Q)\left(\bar{R}_{\mathcal{U}}\right)\right)
$$

This determines a map of $\mathbb{Z}_{p}$-modules $\zeta_{\mathcal{U}}: V_{\mathcal{U}}(\mathbb{L}) \longrightarrow \operatorname{Gr} D(N)(\mathcal{U}) \otimes_{\left(R_{\mathcal{U}} \otimes_{\mathcal{O}_{K}} \mathcal{O}_{M_{0}}\right)} \operatorname{Gr} \mathbb{A}_{\text {cris,M }}(Q)\left(\bar{R}_{\mathcal{U}}\right)$. The composite with the projection onto $V_{\mathcal{U}}(\mathbb{L}) \otimes_{\mathbb{Z}_{p}} B_{\mathrm{HT}}\left(\bar{R}_{\mathcal{U}}\right)$ is the natural map $a \mapsto a \otimes 1$. For every negative integer $m$ extend $\zeta_{\mathcal{U}}$ as a $\operatorname{GrA}_{\text {cris,M }}(m)\left(\bar{R}_{\mathcal{U}}\right)$ linear map

$$
t_{m}\left(\bar{R}_{\mathcal{U}}\right): V_{\mathcal{U}}\left(\mathbb{L}_{)} \otimes_{\mathbb{Z}_{p}} \operatorname{Gr}_{\mathbb{A}_{\text {cris }, \mathrm{M}}}(m)\left(\bar{R}_{\mathcal{U}}\right) \longrightarrow \operatorname{Gr} D(N)(\mathcal{U}) \otimes_{\left(R_{\mathcal{U}} \otimes_{\mathcal{O}_{K}} \mathcal{O}_{M_{0}}\right)} \operatorname{Gr}_{\mathbb{A}_{\text {cris }, \mathrm{M}}}(Q+m)\left(\bar{R}_{\mathcal{U}}\right) .\right.
$$

Then $f_{Q+m}\left(\bar{R}_{\mathcal{U}}\right) \circ t_{m}\left(\bar{R}_{\mathcal{U}}\right)$ is multiplication by $p^{h}$ times the shift by $N+Q$. Similarly, replacing $\zeta_{\mathcal{U}}$ and $t_{m}\left(\bar{R}_{\mathcal{U}}\right)$ with $p^{h} \zeta_{\mathcal{U}}$ and $p^{h} t_{m}\left(\bar{R}_{\mathcal{U}}\right)$, the composite $t_{m+N}\left(\bar{R}_{\mathcal{U}}\right) \circ f_{m}\left(\bar{R}_{\mathcal{U}}\right)$ induces the identity on $\operatorname{Gr} D(N)(\mathcal{U})$ so that $t_{N+m}\left(\bar{R}_{\mathcal{U}}\right) \circ f_{m}\left(\bar{R}_{\mathcal{U}}\right)$ is also $p^{h}$ times the shift by $Q+N$. In particular $t_{m}\left(\bar{R}_{\mathcal{U}}\right)$ and $f_{m}\left(\bar{R}_{\mathcal{U}}\right)$ define inverses one of the other for the two inverse systems defined by varying $m$.

Recall that the composite of $\zeta_{\mathcal{U}}$ with the projection onto $V_{\mathcal{U}}\left(\mathbb{L}_{)} \otimes_{\mathbb{Z}_{p}} B_{\mathrm{HT}}\left(\bar{R}_{\mathcal{U}}\right)\right.$ is the natural map $a \mapsto a \otimes 1$. In particular it is unique with this property and it is $\mathcal{G}_{\mathcal{U}}$-equivariant. This implies 
that multiplying it by $p^{h}$ gives a $\mathcal{G}_{\mathcal{U}}$-equivariant map $\zeta_{\mathcal{U}}$. Note that $\zeta_{U}$ determines $\zeta_{U^{\prime}}$ for any small affine $\mathcal{U}^{\prime} \rightarrow \mathcal{U}$ and since $X$ can be covered by finitely many small affine opens by taking $N$ and $Q$ sufficiently small and $h$ sufficiently large and reducing modulo $p^{n}$ the morphisms $\zeta_{\mathcal{U}}$ glue and define a morphism

$$
\zeta_{n}: \mathbb{L}_{n} \rightarrow \operatorname{Gr} D(N) \otimes_{\left(\mathcal{O}_{X} \otimes_{\mathcal{O}_{K}} \mathcal{O}_{M_{0}}\right)} \operatorname{Gr}\left(\mathbb{A}_{\text {cris,n,M }}^{\prime}(Q)\right)
$$

For every negative integer $m$ we extend it $\operatorname{Gr}_{\text {cris,n,M }}^{\prime}(m)$-linearly to get morphisms

$$
g_{m, n}: \mathbb{L}_{n} \otimes_{\mathbb{Z}_{p}} \operatorname{GrA}_{\text {cris }, \mathrm{n}, \mathrm{M}}^{\prime}(m) \longrightarrow \operatorname{Gr} D(N) \otimes_{\left(\mathcal{O}_{X} \otimes_{\mathcal{O}_{K}} \mathcal{O}_{M_{0}}\right)} \operatorname{Gr}\left(\mathbb{A}_{\text {cris }, \mathrm{n}, \mathrm{M}}^{\prime}(m+Q)\right)
$$

which are compatible for varying $m$ and $n$. Let $\mathcal{U}$ be a small affine. The composite $f_{m+Q, n}\left(\bar{R}_{\mathcal{U}}\right) \circ$ $g_{m, n}\left(\bar{R}_{\mathcal{U}}\right)$ is multiplication by $p^{h}$ on $V_{\mathcal{U}}(\mathbb{L}) / p^{n} V_{\mathcal{U}}(\mathbb{L})=\mathbb{L}_{n}\left(\bar{R}_{\mathcal{U}}\right)$ and hence it is multiplication by $p^{h}$ times the shift by $Q+N$ by linearity. Similarly $g_{m+N, n}\left(\bar{R}_{\mathcal{U}}\right) \circ f_{m, n}\left(\bar{R}_{\mathcal{U}}\right)$ is multiplication by $p^{h}$ on $\operatorname{Gr} D(N)(\mathcal{U})$ and hence it is multiplication by $p^{h}$ times the shift by $N+Q$ by linearity. This implies that $f_{m+Q, n} \circ g_{m, n}$ and $g_{m+N, n}\left(\bar{R}_{\mathcal{U}}\right) \circ c$ are multiplication by $p^{h}$ times the shift by $N+Q$. Thus since multiplication by $p$ and shifts define automorphisms of inductive systems, we conclude that $\left\{f_{m, n}\right\}_{n}$ and $\left\{g_{m, n}\right\}_{n}$ define automorphisms of inductive systems as claimed.

Proposition 3.9. Fix a finite extension $K \subset M$. Assume that $\mathbb{L}$ is a crystalline étale sheaf on $X_{M}^{\mathrm{et}}$ and take $N \in \mathbb{Z}$ as in 3.6. Then we have

1) for varying $r \in \mathbb{N}$ the $\mathcal{O}_{X_{M_{0}}}$-modules $\mathrm{Fil}^{r} \mathbb{D}_{\text {cris }}^{\text {ar }}(\mathbb{L})$ define a decreasing, exhaustive and separated filtration of $\mathrm{Fil}^{r} \mathbb{D}_{\text {cris }}^{\text {ar }}(\mathbb{L})$ by locally free $\mathcal{O}_{X_{M_{0}}}$-modules having the property that the quotient $\mathrm{Fil}^{r} \mathbb{D}_{\text {cris }}^{\text {ar }}(\mathbb{L}) / \mathrm{Fil}^{r+1} \mathbb{D}_{\text {cris }}^{\text {ar }}(\mathbb{L})$ is locally free for every $r \in \mathbb{N}$;

2) the connection $\nabla_{\mathbb{L}}$ on $\mathbb{D}_{\text {cris }}^{\text {ar }}(\mathbb{L})$ is integrable, quasi-nilpotent and satisfies Griffith's transversality relatively to the given filtration;

3) the isomorphism $\alpha_{\text {cris, } \mathbb{L}}: \mathbb{D}_{\text {cris }}^{\text {ar }}(\mathbb{L}) \otimes_{\left(\mathcal{O}_{X} \otimes_{\mathcal{O}_{K}} \mathcal{O}_{M_{0}}\right)} \mathbb{B}_{\text {cris, }, \mathrm{M}} \cong \mathbb{L} \otimes_{\mathbb{Z}_{p}} \mathbb{B}_{\text {cris, }, \mathrm{M}}$ preserves the connection where on the left we consider the composite of the connection $\nabla_{\mathbb{L}}$ and the connection on $\mathbb{B}_{\text {cris, }, \mathrm{M}}$ while on the right we consider the connection induced from the one on $\mathbb{B}_{\text {cris,M }}$ which is trivial on $\mathbb{L}$. Furthermore it induces an isomorphism, in the category $\operatorname{Ind}\left(\operatorname{Sh}\left(\mathfrak{X}_{M}\right)^{\mathbb{N}}\right)$ of inductive systems of continuous sheaves, on filtrations;

4) the map $\mathbb{D}_{\text {cris }}^{\text {ar }}(\mathbb{L}) \widehat{\otimes}_{\mathcal{O}_{M_{0}}} A_{\text {cris }} \longrightarrow \mathbb{D}_{\text {cris }}^{\text {geo }}(\mathbb{L})$ is injective and induces an isomorphism after inverting t. Furthermore $\sum_{a+b=r} \mathrm{Fil}^{a} \mathbb{D}_{\text {cris }}^{\text {ar }}(\mathbb{L}) \widehat{\otimes}_{\mathcal{O}_{M_{0}}} \mathrm{Fil}^{b} A_{\text {cris }}=\left(\mathbb{D}_{\text {cris }}^{\text {ar }}(\mathbb{L}) \widehat{\otimes}_{\mathcal{O}_{M_{0}}} A_{\text {cris }}\right) \cap \mathrm{Fil}^{r} \mathbb{D}_{\text {cris }}^{\text {geo }}(\mathbb{L})$ for every $r \in \mathbb{Z}$.

Proof. 1) follows from [Bri, Prop. 8.3.2].

2) follows from [Bri, Prop. 8.3.4] using [3.6 (which is implicitly assumed in loc. cit.).

3) The assertion regarding the connection is clear. By construction the given morphism preserve the filtrations. Since $X$ is noetherian there exists $H \in \mathbb{Z}$ such that $\mathrm{Fil}^{H} \mathbb{D}_{\text {cris }}^{\text {ar }}(\mathbb{L})=\mathbb{D}_{\text {cris }}^{\text {ar }}(\mathbb{L})$. Due to Lemma 3.1 the fact that $\alpha_{\text {cris, } \mathbb{L}}$ is an isomorphism implies that there exists $N \in \mathbb{N}$

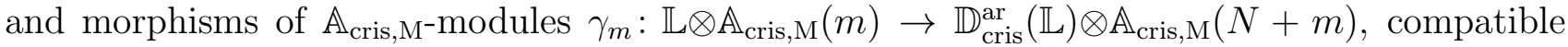

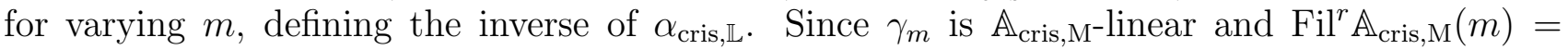
$\left(\mathrm{Fil}^{r-m} \mathbb{A}_{\text {cris,M }}\right) \cdot \mathbb{A}_{\text {cris, }, \mathrm{M}}(m)$ the image via $\gamma_{m}$ of $\mathrm{Fil}^{r}$ on the left hand side is contained in 
$\left(\mathrm{Fil}^{r-m} \mathbb{A}_{\text {cris, } \mathrm{M}}\right) \cdot \mathbb{D}_{\text {cris }}^{\text {ar }}(\mathbb{L}) \otimes \mathbb{A}_{\text {cris }, \mathrm{M}}(N+m)$ which is $\mathbb{D}_{\text {cris }}^{\text {ar }}(\mathbb{L}) \otimes \mathrm{Fil}^{r+N} \mathbb{A}_{\text {cris }, \mathrm{M}}(N+m)$ and is contained in $\mathrm{Fil}^{r+N+H}\left(\mathbb{D}_{\text {cris }}^{\text {ar }}(\mathbb{L}) \otimes \mathbb{A}_{\text {cris }, \mathrm{M}}(N+m)\right)$. In particular $\mathbb{L} \otimes \mathrm{Fil}^{r} \mathbb{B}_{\text {cris,M }}$ is contained in the image of $\mathrm{Fil}^{r+N+H}\left(\mathbb{D}_{\text {cris }}^{\text {ar }}(\mathbb{L}) \otimes \mathbb{B}_{\text {cris, }, \mathrm{M}}\right)$ via $\alpha_{\text {cris }, \mathbb{L}}$.

We are left to prove that the map induced by $\alpha_{\text {cris }, \mathbb{L}}$ on the quotient inductive systems $\mathrm{Fil}^{r} / \mathrm{Fil}^{s}$ for $r \leq s$ is injective. Proceeding inductively it suffices to consider the case that $r=s+1$ and this follows from 3.8 .

4) It follows from $(3)$ that $\mathbb{D}_{\text {cris }}^{\text {geo }}(\mathbb{L})$ is $v_{\bar{K}, *}\left(\mathbb{D}_{\text {cris }}^{\text {ar }}(\mathbb{L}) \otimes_{\left(\mathcal{O}_{X} \otimes_{\mathcal{O}_{K}} \mathcal{O}_{M_{0}}\right)} \mathbb{B}_{\text {cris,M }}\right)$ as filtered module. Since $\mathbb{D}_{\text {cris }}^{\text {ar }}(\mathbb{L})$ is projective as $\mathcal{O}_{X} \otimes_{\mathcal{O}_{K}} \mathcal{O}_{M_{0}}$-module it is locally a direct summand in a free module. To prove the first claim it then suffices to show that for every small affine $\mathcal{U}$, the map $R_{\mathcal{U}} \widehat{\otimes}_{\mathcal{O}_{K}} A_{\text {cris }}\left(\mathcal{O}_{\bar{K}}\right) \longrightarrow\left(A_{\text {cris }}\left(\bar{R}_{\mathcal{U}}\right)^{\mathcal{G}_{\mathcal{U}, \bar{K}}}\right.$ is injective and it has kernel annihilated by a fixed power of $t$. This is proven in [AB, Cor. 31].

To prove the second statement it suffices to show that the map induced on graded pieces $\sum_{a+b=r} \operatorname{Gr}^{a} \mathbb{D}_{\text {cris }}^{\text {ar }}(\mathbb{L}) \widehat{\otimes}_{\mathcal{O}_{M_{0}}} \operatorname{Gr}^{b} A_{\text {cris }} \rightarrow \operatorname{Gr}^{r} \mathbb{D}_{\text {cris }}^{\text {geo }}(\mathbb{L})$ is injective for every $r$. It follows from 3.8 and the fact $\operatorname{Gr}^{a} \mathbb{D}_{\text {cris }}^{\text {ar }}(\mathbb{L})$ is a projective $\mathcal{O}_{X} \otimes_{\mathcal{O}_{K}} \mathcal{O}_{M_{0}}$-module that $v_{\bar{K}, *}\left(\operatorname{Gr}^{r}\left(\mathbb{L} \otimes_{\mathbb{Z}_{p}} \mathbb{B}_{\text {cris,M }}\right)\right)$ is $\left.\bigoplus_{a+b=r} \operatorname{Gr}^{a} \mathbb{D}_{\text {cris }}^{\text {ar }}(\mathbb{L}) \otimes_{\left(\mathcal{O}_{X} \otimes \mathcal{O}_{K}\right.} \mathcal{O}_{M_{0}}\right) v_{\bar{K}, *}\left(\mathrm{Gr}^{b} \mathbb{B}_{\text {cris,M }}\right)$. The claim follows remarking that $\operatorname{Gr}^{b} A_{\text {cris }}$ injects in $v_{\bar{K}, *}\left(\mathrm{Gr}^{b} \mathbb{B}_{\text {cris, } M}\right)$.

Let $\mathcal{U}$ be a small affine and choose parameters $T_{1}, \ldots, T_{d} \in R_{\mathcal{U}}^{\times}$. Write $F_{\mathcal{U}}$ (resp. $\varphi_{\mathcal{U}}$ ) for the associated Frobenius on $\mathcal{U}$ (resp. on $\left.\left.\mathbb{A}_{\text {cris, }, \mathrm{M}}\right|_{\mathfrak{U}_{M}}\right)$. Define a Frobenius $\varphi_{r}$ on $\mathbb{A}_{\text {cris,M }}^{\nabla}(r)$ (resp. $\left.\left.\mathbb{A}_{\text {cris,M }}(r)\right|_{\mathfrak{U}_{M}^{\bullet}}\right)$ to be the map $p^{r} \otimes \varphi$ on $\mathbb{Z}_{p}(r) \otimes_{\mathbb{Z}_{p}} \mathbb{A}_{\text {cris,M }}\left(\operatorname{resp} . \mathbb{Z}_{p}(r) \otimes_{\mathbb{Z}_{p}} \mathbb{A}_{\text {cris,M }} \mid \mathfrak{U}_{M}\right)$. We then get $F_{\mathcal{U}}$-linear maps

$$
\varphi_{\mathcal{U}}: \mathbb{D}_{\text {cris }}^{\text {ar }}(\mathbb{L}) \longrightarrow \mathbb{D}_{\text {cris }}^{\text {ar }}(\mathbb{L}), \quad \varphi_{\mathcal{U}}: v_{M, *}\left(\mathbb{L} \otimes \mathbb{A}_{\text {cris }, \mathrm{M}}(N)\right) \longrightarrow v_{M, *}\left(\mathbb{L} \otimes \mathbb{A}_{\text {cris }, \mathrm{M}}(N)\right) .
$$

Proposition 3.10. Assume that $\mathbb{L}$ is a crystalline étale sheaf. Then we have.

1) $\varphi_{\mathcal{U}}$ is horizontal with respect to the connection $\left.\nabla_{\mathbb{L}}\right|_{\mathcal{U}}$ i.e., $\left.\left.\nabla_{\mathbb{L}}\right|_{\mathcal{U}} \circ \varphi\right|_{\mathcal{U}}=\left.\left(\left.\varphi\right|_{\mathcal{U}} \otimes d F_{\mathcal{U}}\right) \circ \nabla_{\mathbb{L}}\right|_{\mathcal{U}}$,

2) $\left.\mathbb{D}_{\text {cris }}^{\text {ar }}(\mathbb{L})\right|_{\mathcal{U}}$ is an étale $F_{\mathcal{U}}-$ module $i$. e., $\varphi_{\mathcal{U}} \otimes 1:\left.\left.\mathbb{D}_{\text {cris }}^{\text {ar }}(\mathbb{L})\right|_{\mathcal{U}} \otimes_{\mathcal{O}_{\mathcal{U}}}^{F_{\mathcal{U}}} \mathcal{O}_{\mathcal{U}} \rightarrow \mathbb{D}_{\text {cris }}^{\text {ar }}(\mathbb{L})\right|_{\mathcal{U}}$ is an isomorphism.

Proof. (1) follows since $\varphi_{\mathcal{U}}$ on $\mathbb{A}_{\text {cris, } M} \mid \mathcal{U}$ is horizontal by 2.37 ,

$(2)$ follows since $\mathbb{D}_{\text {cris }}^{\text {ar }}(\mathbb{L})$ is a coherent module $\mathbb{D}_{\text {cris }}^{\text {ar }}(\mathbb{L})(\mathcal{U})=D_{\text {cris }}\left(V_{\mathcal{U}}(\mathbb{L})\right)$ and the latter is étale thanks to [Bri, Prop. 8.3.3].

Let $\mathbb{F}$ be the residue field of $\mathcal{O}_{M_{0}}$ and write $\mathcal{U}_{\mathbb{F}}$ for $\mathcal{U} \otimes_{\mathcal{O}_{K}} \mathbb{F}$. Assume that $\mathbb{L}$ is a crystalline étale sheaf on $X_{M}^{\mathrm{et}}$. It follows from $3.9(2)$ and from 3.10 that $\left(\left.\mathbb{D}_{\text {cris }}^{\text {ar }}(\mathbb{L})\right|_{\mathcal{U}}, \varphi_{\mathcal{U}}\right)$ is a convergent $F$-isocrystal $\mathcal{U}_{\mathbb{F}}$ relatively to $\mathcal{O}_{M_{0}}$ in the sense of [B3, Def. 2.3.7].

Lemma 3.11. Suppose we have two choices of parameters $T_{1}, \ldots, T_{d}$ and $T_{1}^{\prime}, \ldots, T_{d}^{\prime}$ of $R_{\mathcal{U}}^{\times}$. Denote by $\varphi_{\mathcal{U}}$ and $\varphi_{\mathcal{U}}^{\prime}$ the corresponding Frobenius morphisms on $\left.\mathbb{D}_{\text {cris }}^{\text {ar }}(\mathbb{L})\right|_{\mathcal{U}}$. Then $\left(\left.\mathbb{D}_{\text {cris }}^{\text {ar }}(\mathbb{L})\right|_{\mathcal{U}}, \varphi_{\mathcal{U}}\right)$ and $\left(\left.\mathbb{D}_{\text {cris }}^{\text {ar }}(\mathbb{L})\right|_{\mathcal{U}}, \varphi_{\mathcal{U}}^{\prime}\right)$ define the same convergent $F$-crystals on $\mathcal{U}_{\mathbb{F}}$ relatively to $\mathcal{O}_{M_{0}}$.

Proof. Let $F_{\mathcal{U}}$ and $F_{\mathcal{U}}^{\prime}$ be the Frobenii on $R_{\mathcal{U}}$ defined by the two choices of parameters. Let $p_{1}$, $p_{2}: \mathcal{U} \times_{\mathcal{O}_{K}} \mathcal{U} \rightarrow \mathcal{U}$ be the two projections. The convergent connection $\nabla_{\mathbb{L}}$ on $\mathbb{D}:=\left.\mathbb{D}_{\text {cris }}^{\text {ar }}(\mathbb{L})\right|_{\mathcal{U}}$ defines an isomorphism of $\mathcal{O}_{\mathcal{U}_{K}}$-modules $\epsilon: p_{2}^{*}(\mathbb{D}) \longrightarrow p_{1}^{*}(\mathbb{D})$ on the tube of the diagonal $\mathcal{U} \rightarrow$ 
$\mathcal{U} \times \mathcal{U}$ defining the structure of isocrystal on $\mathbb{D}$. Consider $F=\left(F_{\mathcal{U}}, F_{\mathcal{U}}^{\prime}\right): \mathcal{U}_{K} \rightarrow \mathcal{U}_{K} \times \mathcal{U}_{K}$. Then $F^{*}(\epsilon)$ induces an isomorphism $F_{\mathcal{U}}^{*}(\mathbb{D}) \longrightarrow F_{\mathcal{U}}^{*}(\mathbb{D})$. The claim amounts to prove that $\varphi_{\mathcal{U}}^{\prime} \otimes 1=\varphi_{\mathcal{U}} \otimes 1 \circ F^{*}(\varepsilon)$. Since $\mathbb{D}$ is coherent it suffices to verify this on $\mathcal{U}_{K}$-sections. Write $D:=\mathbb{D}\left(\mathcal{U}_{K}\right)$. The map $\epsilon$ on $\mathcal{U}$ is the map $D \ni m \mapsto \sum_{\underline{n} \in \mathbb{N}^{d}}\left(\prod_{i=1}^{d} N_{i}^{n_{i}}\right)(m) \otimes\left(1 \otimes T_{i}-T_{i} \otimes 1\right)^{\left[n_{i}\right]}$ where $N_{i}$ is the endomorphism of $D$ given by $\nabla_{\mathbb{L}} \circ 1 \otimes \frac{\partial}{\partial T_{i}}$ see [BO, Pf. Thm. 4.12]. Thus $F^{*}(\epsilon)$ is the map sending $m \otimes 1 \in D \otimes_{R_{\mathcal{U}}}^{F_{\mathcal{U}}^{\prime}} R_{\mathcal{U}}$ to $\sum_{\underline{n} \in \mathbb{N}^{d}}\left(\prod_{i=1}^{d} N_{i}^{n_{i}}\right)(m) \otimes\left(F_{\mathcal{U}}^{\prime}\left(T_{i}\right)-F_{\mathcal{U}}\left(T_{i}\right)\right)^{\left[n_{i}\right]}$. Eventually $\varphi_{\mathcal{U}} \otimes 1 \circ F^{*}(\epsilon)$ is the map $m \mapsto \sum_{\underline{n} \in \mathbb{N}^{d}} \varphi_{\mathcal{U}}\left(\prod_{i=1}^{d} N_{i}^{n_{i}}\right)(m) \otimes\left(F_{\mathcal{U}}^{\prime}\left(T_{i}\right)-F_{\mathcal{U}}\left(T_{i}\right)\right)^{\left[n_{i}\right]}$. This is the expression for $\varphi_{\mathcal{U}}^{\prime}$ computed in [Bri, Prop. 7.2.3].

In particular the $F$-isocrystals defined by $\left(\left.\mathbb{D}_{\text {cris }}^{\text {ar }}(\mathbb{L})\right|_{\mathcal{U}}, \varphi_{\mathcal{U}}\right)$ glue for different choices of $\mathcal{U}$ 's and parameters and define a convergent $F$-isocrystal $\left(\mathbb{D}_{\text {cris }}^{\text {ar }}(\mathbb{L}), \nabla_{\mathbb{L}}, \varphi_{\mathbb{L}, M}\right)$. Denote by $\operatorname{Isoc}\left(X_{\mathbb{F}} / M_{0}\right)$ the category of filtered convergent $F$-isocrystals. It is a tensor category and it is abelian if we consider only convergent $F$-isocrystals (forgetting the filtrations); see [B3, Rmk. 2.3.3(iii)\&§2.3.7]. For every $n \in \mathbb{Z}$ define $\mathbf{1}(n)$ to be the isocrystal $\mathcal{O}_{X_{M_{0}}}$ with the connection defined by the usual derivation, Frobenius given by $p^{-n}$ times the Frobenius on $\mathcal{O}_{X_{M_{0}}}$ and filtration which is 0 for $r>n$ and is $\mathcal{O}_{X_{M_{0}}}$ for $r \leq n$. Given a convergent filtered $F$-isocrystal $\mathcal{E}$ we put $\mathcal{E}(n):=\mathcal{E} \otimes \mathbf{1}(n)$ and we call it the $n$-th Tate twist of $\mathcal{E}$. We get a functor

$$
\mathbb{D}_{\text {cris }}^{\text {ar }}: \operatorname{Sh}\left(X_{M}^{\mathrm{et}}\right)_{\mathbb{Q}_{p}}^{\text {cris }} \longrightarrow \operatorname{IsOc}\left(X_{\mathbb{F}} / M_{0}\right)
$$

given by

$$
\mathbb{L} \mapsto\left(\mathbb{D}_{\text {cris }}^{\text {ar }}(\mathbb{L}), \nabla_{\mathbb{L}},\left\{\mathrm{Fil}^{r} \mathbb{D}_{\text {cris }}^{\text {ar }}(\mathbb{L})\right\}, \varphi_{\mathbb{L}, M}\right) .
$$

Define Isoc $\left(X_{\mathbb{F}} / M_{0}\right)^{\mathrm{adm}}$, the category of admissible filtered convergent $F$-isocrystals, to be the essential image of $\mathbb{D}_{\text {cris }}^{\text {ar }}$.

Let $\underline{\mathcal{E}}:=\left((\mathcal{E}, \nabla),\left\{\mathrm{Fil}^{r} \mathcal{E}\right\}_{r \in \mathbb{Z}}, \Phi\right)$ be a filtered convergent $F$-isocrystal on $X_{\mathbb{F}}$ relative to $M_{0}$. Due to [B3, Thm. 2.4.2] there exists an $\mathcal{O}_{X} \otimes_{\mathcal{O}_{K}} \mathcal{O}_{M_{0}}-$ module $(\mathcal{M}, \nabla, \Phi)$ with integrable and nilpotent connection and non-degenerate Frobenius such that $\left(\mathcal{M}^{\text {rig }}, \nabla^{\text {rig }}, \Phi^{\text {rig }}\right)$ is the Tate twist $(\mathcal{E}(n), \nabla, \Phi(n))$ for some $n \in \mathbb{N}$. By loc. cit. such crystal is unique up to isogeny and up to Tate twist. Define

$$
\mathbb{V}_{\text {cris }}^{\mathrm{ar}}(\underline{\mathcal{E}}):=\operatorname{Fil}^{0}\left(v_{M}^{*}(\mathcal{M})(-n) \otimes_{\mathcal{O}_{\mathfrak{X}_{M}}^{\text {un }} \otimes_{\mathcal{O}_{K}} \mathcal{O}_{M_{0}}} \mathbb{A}_{\text {cris, }, \mathrm{K}}\right)^{\nabla=0, \Phi=1} \in \operatorname{Ind}\left(\operatorname{Sh}\left(\mathfrak{X}_{M}\right)^{\mathbb{N}}\right)
$$

Recall that we have a fully faithful functor $u_{X, M, *}: \operatorname{Sh}\left(X_{M}^{\mathrm{et}}\right)_{\mathbb{Q}_{p}} \longrightarrow \operatorname{Ind}\left(\operatorname{Sh}\left(\mathfrak{X}_{M}\right)^{\mathbb{N}}\right)$ and we identify $\operatorname{Sh}\left(X_{M}^{\mathrm{et}}\right)_{\mathbb{Q}_{p}}$ with its essential image.

Theorem 3.12. The following hold:

1) the sub-category $\operatorname{Sh}\left(X_{M}^{\mathrm{et}}\right)_{\mathbb{Q}_{p}}^{\text {cris }}$ of $\operatorname{Sh}\left(X_{M}^{\mathrm{et}}\right)^{\mathbb{N}}$ is an abelian tensor sub-category, closed under Tate twists, duals and tensor products;

2) the sub-category $\operatorname{Isoc}\left(X_{\mathbb{F}} / M_{0}\right)^{\text {adm }}$ of admissible filtered convergent $F$-isocrystals of $\operatorname{Isoc}\left(X_{\mathbb{F}} / M_{0}\right)$ is an abelian tensor sub-category, closed under Tate twists, duals and tensor products.

3) the functor $\mathbb{D}_{\text {cris }}^{\text {ar }}$ is an exact functor of abelian tensor categories, it is fully faithful and commutes with duals and Tate twists; 
4) the functor $\mathbb{V}_{\text {cris }}^{\mathrm{ar}}$ factors via $S h\left(X_{M}^{\mathrm{et}}\right)_{\mathbb{Q}_{p}}^{\mathrm{cris}}$ and $\mathbb{V}_{\text {cris }}^{\mathrm{ar}} \circ \mathbb{D}_{\text {cris }}^{\mathrm{ar}}$ is equivalent to the identity. In particular $\mathbb{D}_{\text {cris }}^{\text {ar }}$ defines an equivalence of categories

$$
\operatorname{Sh}\left(X_{M}^{\mathrm{et}}\right)_{\mathbb{Q}_{p}}^{\mathrm{cris}} \cong \operatorname{ISOc}\left(X_{\mathbb{F}} / M_{0}\right)^{\mathrm{adm}}
$$

Proof. Claim (2) follows from (1)\&(3). By construction $\mathbb{D}_{\text {cris }}^{\text {ar }}$ is essentially surjective. To verify (1) and the rest of (3) one reduces to the case that $X=\mathcal{U}$ is small affine. To verify that $\operatorname{Sh}\left(X_{M}^{\text {et }}\right)_{\mathbb{Q}_{p}}^{\text {cris }}$ is closed under tensor product, internal Hom and Tate twists and that $\mathbb{D}_{\text {cris }}^{\text {ar }}$ commutes with tensor products, internal Hom and Tate twists one reduces to the case that $X=\mathcal{U}$ is a small affine. Using that $\mathbb{D}_{\text {cris }}^{\text {ar }}(\mathbb{L})(\mathcal{U})=D_{\text {cris }}\left(V_{\mathcal{U}}(\mathbb{L})\right)$ and that $\mathbb{D}_{\text {cris }}^{\text {ar }}(\mathbb{L})$ is coherent, these claims follow from analogous statements for $D_{\text {cris }}\left(V_{\mathcal{U}}(\mathbb{L})\right)$ proven in [Bri, Thm. 8.4.2].

It follows from loc. cit. that if $\mathbb{L}$ is crystalline and $\mathcal{U}$ is a small affine as in 3.7 one can recover the $\mathbb{Q}_{p}$-representation $V_{\mathcal{U}}(\mathbb{L})$ from $D_{\text {cris }}\left(V_{\mathcal{U}}(\mathbb{L})\right)$ by the formula

$$
V_{\mathcal{U}}(\mathbb{L})=\operatorname{Fil}^{0}\left(D_{\text {cris }}\left(V_{\mathcal{U}}(\mathbb{L})\right) \otimes_{R_{\mathcal{U}} \otimes_{\mathcal{O}_{K}} \mathcal{O}_{M_{0}}} B_{\text {cris }}\left(\bar{R}_{\mathcal{U}}\right)\right)^{\nabla=0, \varphi=1}
$$

This is equal to $\mathbb{V}_{\text {cris }}^{\text {ar }}\left(\mathbb{D}_{\text {cris }}^{\text {ar }}(\mathbb{L})\right)\left(\bar{R}_{\mathcal{U}}\right)\left[p^{-1}\right]$ thanks to 3.2. This allows to recover $\mathbb{L}$ up to isogeny. In particular $\mathbb{D}_{\text {cris }}^{\text {ar }}$ is fully faithful. Being essentially surjective it defines an equivalence of categories.

It is a difficult question to characterize $\operatorname{Isoc}\left(X_{\mathbb{F}} / M_{0}\right)^{\text {adm }}$ in $\operatorname{Isoc}\left(X_{\mathbb{F}} / M_{0}\right)$. If $X=\operatorname{Spf}\left(\mathcal{O}_{K}\right)$, a satisfactory answer is provided in $[\mathrm{CF}]$ in terms of the so called weakly admissible modules. In more generality a complete answer exists for admissible filtered convergent $F$-isocrystals of rank 1 thanks to Bri]. Let $\underline{\mathcal{E}}:=\left((\mathcal{E}, \nabla),\left\{\mathrm{Fil}^{r} \mathcal{E}\right\}_{r \in \mathbb{Z}}, \Phi\right)$ be a filtered convergent $F$-isocrystal on $X_{\mathbb{F}}$ relative to $M_{0}$ of rank 1 i. e., $\mathcal{E}$ is a locally free $\mathcal{O}_{X_{K}}$ rig - module of rank 1 . Define $t_{H}(\mathcal{E})$ to be the locally constant function on $X \otimes_{\mathcal{O}_{K}} \mathcal{O}_{M_{0}}$ locally defined as the largest integer such that $\mathrm{Fil}^{r} \mathcal{E}=\mathcal{E}$.

Let $\mathcal{U}=\operatorname{Spf}\left(R_{\mathcal{U}}\right)$ be a small étale open affine of $X$ such that $\left.\mathcal{E}\right|_{\mathcal{U}}=\mathcal{O}_{\mathcal{U}} \otimes_{\mathcal{O}_{K}} M_{0}$ e. Since $\mathcal{E}$ is an isocrystal we have $\Phi(e)=a_{\mathcal{U}} e$ with $a_{\mathcal{U}} \in R_{\mathcal{U}} \otimes_{\mathcal{O}_{K}} \mathcal{O}_{M_{0}}\left[p^{-1}\right]^{\times}$. For every connected component $\mathcal{U}_{i}=\operatorname{Spec}\left(R_{\mathcal{U}_{i}}\right)$ of $\operatorname{Spec}\left(R_{\mathcal{U}} \otimes_{\mathcal{O}_{K}} \mathcal{O}_{M_{0}}\right)$, the element $p$ generates a prime ideal of $R_{\mathcal{U}_{i}}$ and we must have $a_{\mathcal{U}}=p^{n_{i}} \alpha$ with $\alpha_{\mathcal{U}_{i}}$ a unit in $R_{\mathcal{U}_{i}}$. Then the integer $t_{N}(\mathcal{E})\left(\mathcal{U}_{i}\right):=n_{i}$ does not depend on the choice of $e$ and is locally constant on $X \otimes_{\mathcal{O}_{K}} \mathcal{O}_{M_{0}}$. Then we have.

Proposition 3.13. A rank 1 , filtered convergent $F$-isocrystal $\underline{\mathcal{E}}$ is admissible if and only if is locally free as $\mathcal{O}_{X} \otimes_{\mathcal{O}_{K}} M_{0}$ for the étale topology on $X$ and we have $t_{H}(\mathcal{E})=t_{N}(\mathcal{E})$.

Proof. It follows from 3.7 and [Bri, Prop. 8.6.2].

Finally we compare our notion of a crystalline étale sheaf with the notion of "associated sheaves" given in [F2, p. 67]. Let $\underline{\mathcal{E}}$ be a filtered convergent $F$-isocrystal on $X_{\mathbb{F}}$ relative to $M_{0}$. As explained above we may assume that up to Tate twist it is the generic fiber of a $\mathcal{O}_{X} \otimes_{\mathcal{O}_{K}} \mathcal{O}_{M_{0}}$-module $\mathcal{M}$ endowed with an integrable and nilpotent connection and a nondegenerate Frobenius. We identify $\mathcal{M}$ with the associated crystal on $X_{\mathbb{F}} / \mathcal{O}_{M_{0}}$. Given a small affine $\mathcal{U}:=\operatorname{Spf}\left(R_{\mathcal{U}}\right)$ of $X$ we write $\mathcal{E}\left(B_{\text {cris }}^{\nabla}\left(\bar{R}_{\mathcal{U}}\right)\right)$ for $\mathcal{M}\left(A_{\text {cris }}^{\nabla}\left(\bar{R}_{\mathcal{U}}\right)\right) \otimes_{A_{\text {cris }}} B_{\text {cris }}$ where $\mathcal{M}\left(A_{\text {cris }}^{\nabla}\left(\bar{R}_{\mathcal{U}}\right)\right)$ is the value of the crystal $\mathcal{M}$ on the PD-thickening $\theta: A_{\text {cris }}^{\nabla}\left(\bar{R}_{\mathcal{U}}\right) \rightarrow \widehat{\bar{R}}_{\mathcal{U}}$. Note that given a morphism $\sigma: R_{\mathcal{U}} \otimes_{\mathcal{O}_{K}} \mathcal{O}_{M_{0}} \rightarrow A_{\text {cris }}^{\nabla}\left(\bar{R}_{\mathcal{U}}\right)$ as $\mathcal{O}_{M_{0}}$-algebras inducing the identity on $R_{\mathcal{U}} \otimes_{\mathcal{O}_{K}} \mathcal{O}_{M_{0}}$ via 
the projection $A_{\text {cris }}^{\nabla}\left(\bar{R}_{\mathcal{U}}\right) \rightarrow \widehat{\bar{R}}_{\mathcal{U}}$ (for example the one sending $\left.T_{i} \mapsto \widetilde{T}_{i}\right)$ we get that $\mathcal{E}\left(B_{\text {cris }}^{\nabla}\left(\bar{R}_{\mathcal{U}}\right)\right) \cong$ $\mathcal{M}\left(R_{\mathcal{U}}\right) \otimes_{R_{\mathcal{U}} \otimes_{\mathcal{O}_{K}} \mathcal{O}_{M_{0}}} B_{\text {cris }}^{\nabla}\left(\bar{R}_{\mathcal{U}}\right) \cong \mathcal{E}\left(R_{\mathcal{U}}\right) \otimes_{R_{\mathcal{U}} \otimes_{\mathcal{O}_{K}} \mathcal{O}_{M_{0}}} B_{\text {cris }}^{\nabla}\left(\bar{R}_{\mathcal{U}}\right)$. Since $\mathcal{M}$ is a crystal the first identification does not depend on the choice of $\sigma$. More precisely, given two sections $\sigma$ and $\sigma^{\prime}$ there is a canonical isomorphism between $\mathcal{M}\left(R_{\mathcal{U}}\right) \otimes_{R_{\mathcal{U}} \otimes_{\mathcal{O}_{K}} \mathcal{O}_{M_{0}}}^{\sigma} B_{\text {cris }}^{\nabla}\left(\bar{R}_{\mathcal{U}}\right)$ and $\mathcal{M}\left(R_{\mathcal{U}}\right) \otimes_{R_{\mathcal{U}} \otimes_{\mathcal{O}_{K}} \mathcal{O}_{M_{0}}}^{\sigma_{\text {cris }}} B_{R_{\mathcal{U}}}^{\nabla}$ whose Taylor expansion is defined using the connection on $\mathcal{M}$. In particular $\mathcal{E}\left(B_{\text {cris }}^{\nabla}\left(\bar{R}_{\mathcal{U}}\right)\right)$ is endowed with an action of $\mathcal{G}_{\mathcal{U}, M}$ and Frobenius and since the connection on $\mathcal{E}$ satisfies Griffith's transversality the filtration on $\mathcal{E}\left(B_{\text {cris }}^{\nabla}\left(\bar{R}_{\mathcal{U}}\right)\right)$ induced from the filtration on $\mathcal{E}\left(R_{\mathcal{U}}\right)$ does not depend on $\sigma$. Let $\mathbb{L}$ be a $\mathbb{Q}_{p}$-adic étale sheaf on $X_{M}$. Following Faltings one says that $\mathbb{L}$ and $\mathcal{E}$ are associated if there is an isomorphism

$$
\rho_{\mathcal{U}}: \mathcal{E}\left(B_{\text {cris }}^{\nabla}\left(\bar{R}_{\mathcal{U}}\right)\right) \cong V_{\mathcal{U}}\left(\mathbb{L}_{)} \otimes_{\mathbb{Z}_{p}} B_{\text {cris }}^{\nabla}\left(\bar{R}_{\mathcal{U}}\right)\right.
$$

of $B_{\text {cris }}^{\nabla}\left(\bar{R}_{\mathcal{U}}\right)$-modules commuting with filtrations action of $\mathcal{G}_{\mathcal{U}, M}$ and Frobenius for every small affine $\mathcal{U}$ which is functorial in $\mathcal{U}$. Recall that $V_{\mathcal{U}}(\mathbb{L})$ is the $\mathcal{G}_{\mathcal{U}, M}$-representation $\mathbb{L}\left(\bar{R}_{\mathcal{U}}\right)$. Then we have.

Lemma 3.14. The sheaves $\underline{\mathcal{E}}$ and $\mathbb{L}$ are associated in Faltings' sense if and only if $\mathbb{L}$ is crystalline and $\mathbb{D}_{\text {cris }}^{\text {ar }}(\mathbb{L})=\underline{\mathcal{E}}$.

Proof. First of all we remark that to be associated in Faltings' sense it suffices that there is a covering of $X$ by small affines $\left\{\mathcal{U}_{i}\right\}_{i}$ such that we have an isomorphism $\rho_{\mathcal{U}_{i}}$ for every $i$ and $\rho_{\mathcal{U}_{i}}$ and $\rho_{\mathcal{U}_{j}}$ are compatible on $\mathcal{U}_{i} \cap \mathcal{U}_{j}$. By 3.7 we have that $\mathbb{L}$ is crystalline if and only if there is a covering of $X$ by small affines such that the natural map $g_{\mathcal{U}_{i}}: \mathbb{D}_{\text {cris }}^{\text {ar }}(\mathbb{L})\left(\mathcal{U}_{i}\right) \otimes_{R_{\mathcal{U}_{i}} \otimes_{\mathcal{O}_{K}} \mathcal{O}_{M_{0}}} B_{\text {cris }}\left(\bar{R}_{\mathcal{U}_{i}}\right) \cong$ $V_{\mathcal{U}_{i}}(\mathbb{L}) \otimes_{\mathbb{Z}_{p}} B_{\text {cris }}\left(\bar{R}_{\mathcal{U}_{i}}\right)$ is an isomorphism. By construction $g_{\mathcal{U}_{i}}$ is an isomorphism of $B_{\text {cris }}\left(\bar{R}_{\mathcal{U}}\right)-$ modules and it commutes with filtrations, action of $\mathcal{G}_{\mathcal{U}, M}$, Frobenius and connections. For every $i$ take the section $\sigma_{i}: R_{\mathcal{U}_{i}} \otimes_{\mathcal{O}_{K}} \mathcal{O}_{M_{0}} \rightarrow A_{\text {cris }}^{\nabla}\left(\bar{R}_{\mathcal{U}_{i}}\right)$ sending $T_{j} \rightarrow \widetilde{T}_{j}$. It induces the section of the inclusion $A_{\text {cris }}^{\nabla}\left(\bar{R}_{\mathcal{U}_{i}}\right) \rightarrow A_{\text {cris }}\left(\bar{R}_{\mathcal{U}_{i}}\right) \cong A_{\text {cris }}^{\nabla}\left(\bar{R}_{\mathcal{U}_{i}}\right)\left\langle 1 \otimes T_{j}-\widetilde{T}_{j} \otimes 1\right\rangle_{j=1, \ldots, d}$ sending $1 \otimes T_{j}-\widetilde{T}_{j} \otimes 1 \mapsto 0$. Therefore it defines a section $\widetilde{\sigma}_{i}$ of $B_{\text {cris }}^{\nabla}\left(\bar{R}_{\mathcal{U}_{i}}\right) \rightarrow B_{\text {cris }}\left(\bar{R}_{\mathcal{U}_{i}}\right)$ compatible with filtrations, Frobenius and $\mathcal{G}_{\mathcal{U}, M}$-action (considering on $B_{\text {cris }}\left(\bar{R}_{\mathcal{U}_{i}}\right)$ the Galois action twisted via the connection).

If $\mathbb{L}$ is crystalline then we get an isomorphism $\rho_{\mathcal{U}_{i}}:=\widetilde{\sigma}_{i}^{*}\left(g_{\mathcal{U}_{i}}\right)$, compatible with all the supplementary structures and $\mathbb{L}$ and $\mathbb{D}_{\text {cris }}^{\text {ar }}(\mathbb{L})$ are associated. In the other direction, assume that $\mathbb{L}$ and $\mathcal{E}$ are associated. Write $\mathcal{E}\left(B_{\text {cris }}\left(\bar{R}_{\mathcal{U}_{i}}\right)\right)$ for $\mathcal{M}\left(A_{\text {cris }}\left(\bar{R}_{\mathcal{U}}\right)\right) \otimes_{A_{\text {cris }}} B_{\text {cris }}$ where $\mathcal{M}\left(A_{\text {cris }}\left(\bar{R}_{\mathcal{U}}\right)\right)$ is the value of the crystal $\mathcal{M}$ on the PD-thickening $A_{\text {cris }}\left(\bar{R}_{\mathcal{U}}\right) \rightarrow \widehat{\bar{R}}_{\mathcal{U}}$. Since $\mathcal{M}$ is a crystal by definition [BO, Def. 6.1] we have canonical isomorphisms $\mathcal{E}\left(B_{\text {cris }}\left(\bar{R}_{\mathcal{U}_{i}}\right)\right) \cong \mathcal{E}(\mathcal{U}) \otimes_{R_{\mathcal{U}} \otimes_{\mathcal{O}_{K}} \mathcal{O}_{M_{0}}} B_{\text {cris }}\left(\bar{R}_{\mathcal{U}_{i}}\right)$ and $\mathcal{E}\left(B_{\text {cris }}\left(\bar{R}_{\mathcal{U}_{i}}\right)\right) \cong \mathcal{E}\left(B_{\text {cris }}^{\nabla}\left(\bar{R}_{\mathcal{U}_{i}}\right)\right) \otimes_{B_{\text {cris }}^{\nabla}\left(\bar{R}_{\mathcal{U}_{i}}\right)} B_{\text {cris }}\left(\bar{R}_{\mathcal{U}_{i}}\right)$ of $B_{\text {cris }}\left(\bar{R}_{\mathcal{U}_{i}}\right)$-modules. Being canonical they commute with the $\mathcal{G}_{\mathcal{U}, M^{-}}$-action and Frobenius. Since the connection on $\mathcal{E}$ satisfies Griffith's transversality the isomorphisms preserve also the filtrations. Define $g_{\mathcal{U}_{i}}$ to be the extension of scalars of $\rho_{\mathcal{U}_{i}}$ using the second isomorphism. It follows from the first isomorphism that $\mathbb{D}_{\text {cris }}^{\text {ar }}(\mathbb{L})=\mathcal{E}$ as filtered convergent Frobenius isocrystal. In particular $\mathbb{L}$ is crystalline. The claim follows.

\subsection{The cohomology of crystalline sheaves.}

As before we assume that $\mathcal{O}_{K}=\mathbb{W}(k)$ and we fix a finite extension $K \subset M$ contained in $\bar{K}$. Let $X$ be a smooth $p$-adic formal scheme over $\mathcal{O}_{K}$. 
Theorem 3.15. We have canonical isomorphisms of $\delta$-functors from $S h\left(X_{M}^{\mathrm{et}}\right)_{\mathbb{Q}_{p}}^{\text {cris }}$ to the category of filtered $B_{\text {cris }}$-modules endowed with the action of $G_{M}$ and Frobenius

$$
\mathrm{H}^{i}\left(\mathfrak{X}_{\bar{K}}, \mathbb{L} \otimes \mathbb{B}_{\text {cris }, \overline{\mathrm{K}}}^{\nabla}\right) \cong \mathrm{H}_{\mathrm{dR}}^{i}\left(X^{\mathrm{et}}, \mathbb{D}_{\text {cris }}^{\text {geo }}(\mathbb{L})\right),
$$

for $\mathbb{L}$ a crystalline $\mathbb{Q}_{p}$-adic étale sheaf. In fact for every $r \in \mathbb{Z}$ we have isomorphisms of $B_{\text {cris }}$-modules which are $G_{M}$-equivariant and compatible for varying $r$ 's and compatible with the previous isomorphism

$$
\mathrm{H}^{i}\left(\mathfrak{X}_{\bar{K}}, \mathbb{L} \otimes \operatorname{Fil}^{r} \mathbb{B}_{\text {cris }, \overline{\mathrm{K}}}^{\nabla}\right) \cong \mathbb{H}^{i}\left(X^{\mathrm{et}}, \mathrm{Fil}^{\mathrm{r}-\bullet} \mathbb{D}_{\text {cris }}^{\text {geo }}(\mathbb{L}) \otimes_{\mathcal{O}_{X}} \Omega_{X / \mathcal{O}_{K}}^{\bullet}\right)
$$

The cohomology $\mathrm{H}^{i}\left(\mathfrak{X}_{\bar{K}}, \mathbb{L} \otimes \mathrm{Fil}^{r} \mathbb{B}_{\text {cris }, \overline{\mathrm{K}}}^{\nabla}\right)$ of the inductive system $\mathbb{L} \otimes \mathrm{Fil}^{r} \mathbb{B}_{\text {cris }, \overline{\mathrm{K}}}^{\nabla}$ is taken as explained in $\$ 2.8$ for every $r \in \mathbb{Z} \cup\{-\infty\}$.

The cohomology group $\mathbb{H}^{i}\left(X^{\mathrm{et}}, \mathrm{Fil}^{\mathrm{r}-\bullet} \mathbb{D}_{\text {cris }}^{\text {geo }}(\mathbb{L}) \otimes_{\mathcal{O}_{X}} \Omega_{X / \mathcal{O}_{K}}^{\bullet}\right)$ means the following. Recall that for every $r \in \mathbb{Z}$ we have a complex of sheaves on $X$ denoted $\mathrm{Fil}^{\mathrm{r}-\bullet} \mathbb{D}_{\text {cris }}^{\text {geo }}(\mathbb{L}) \otimes_{\mathcal{O}_{X}} \Omega_{X / \mathcal{O}_{K}}^{\bullet}$ and given by

$$
\mathrm{Fil}^{r} \mathbb{D}_{\text {cris }}^{\text {geo }}(\mathbb{L}) \stackrel{\nabla}{\longrightarrow} \mathrm{Fil}^{r-1} \mathbb{D}_{\text {cris }}^{\text {geo }}(\mathbb{L}) \otimes_{\mathcal{O}_{X}} \Omega_{X / \mathcal{O}_{K}}^{1} \stackrel{\nabla}{\longrightarrow} \mathrm{Fil}^{r-2} \mathbb{D}_{\text {cris }}^{\text {gee }}(\mathbb{L}) \otimes_{\mathcal{O}_{X}} \Omega_{X / \mathcal{O}_{K}}^{2} \stackrel{\nabla}{\longrightarrow} \ldots
$$

We denote by $\mathbb{H}^{i}\left(X^{\mathrm{et}}, \mathrm{Fil}^{\mathrm{r}-\bullet} \mathbb{D}_{\text {cris }}^{\text {geo }}(\mathbb{L}) \otimes_{\mathcal{O}_{X}} \Omega_{X / \mathcal{O}_{K}}^{\bullet}\right)$ the $i$-th hypercohomology group of the respective complex.

The filtrations: For $r \in \mathbb{Z}$ the $r$-th filtration of $\mathrm{H}_{\mathrm{dR}}^{i}\left(X^{\mathrm{et}}, \mathbb{D}_{\text {cris }}^{\text {geo }}(\mathbb{L})\right)$ is by definition the image of $\mathbb{H}^{i}\left(X^{\mathrm{et}}, \mathrm{Fil}^{\mathrm{r}-\bullet} \mathbb{D}_{\text {cris }}^{\text {geo }}(\mathbb{L}) \otimes_{\mathcal{O}_{X}} \Omega_{X / \mathcal{O}_{K}}^{\bullet}\right)$ while the $r$-the filtration on $\mathrm{H}^{i}\left(\mathfrak{X}_{\bar{K}}, \mathbb{L} \otimes_{\mathbb{Z}_{p}} \mathbb{B}_{\text {cris }}^{\nabla}\right)$ is the image of $\left\{\mathrm{H}^{i}\left(\mathfrak{X}_{\bar{K}}, \mathbb{L} \otimes_{\mathbb{Z}_{p}} \mathrm{Fil}^{r} \mathbb{B}_{\text {cris }}^{\nabla}\right)\right\}_{r}$.

Galois action: The Galois action on $\mathrm{H}_{\mathrm{dR}}^{i}\left(X^{\mathrm{et}}, \mathrm{Fil}^{r} \mathbb{D}_{\text {cris }}^{\text {geo }}(\mathbb{L})\right)$ is induced by the Galois action on $\mathbb{D}_{\text {cris }}^{\text {geo }}(\mathbb{L})$ defined in 3.3 . The Galois action on $\mathrm{H}^{i}\left(\mathfrak{X}_{\bar{K}}, \mathbb{L} \otimes \operatorname{Fil}^{r} \mathbb{B}_{\text {cris, } \overline{\mathrm{K}}}^{\nabla}\right)$ arises as follows. Since $\beta_{M, \bar{K}}^{*}$ is exact and sends flasque objects to flasque objects by 2.14 and [AI, Pf. Prop. 4.4(4)] and since $\beta_{M, \bar{K}}^{*}\left(\mathbb{L} \otimes \mathrm{Fil}^{r} \mathbb{A}_{\text {cris,M }}^{\nabla}\right)=\mathbb{L} \otimes \mathrm{Fil}^{r} \mathbb{A}_{\text {cris }, \overline{\mathrm{K}}}^{\nabla}$, one can compute $\mathrm{H}^{i}\left(\mathfrak{X}_{\bar{K}}, \mathbb{L} \otimes \mathrm{Fil}^{r} \mathbb{A}_{\text {cris }, \overline{\mathrm{K}}}^{\nabla}\right)$ taking global sections of the pull-back via $\beta_{M, \bar{K}}^{*}$ of an injective resolution $\mathcal{I}^{\bullet}$ of $\mathbb{L} \otimes \mathrm{Fil}^{r} \mathbb{A}_{\text {cris, } \mathrm{K}}^{\nabla}$. Since $v_{\bar{K}, *}\left(\beta_{M, \bar{K}}^{*}\left(\mathcal{I}^{\bullet}\right)\right)=v_{M, *}\left(\beta_{K, \bar{K}, *} \circ \beta_{M, \bar{K}}^{*}\left(\mathcal{I}^{\bullet}\right)\right)$ and $\beta_{M, \bar{K}, *} \circ \beta_{M, \bar{K}}^{*}\left(\mathcal{I}^{\bullet}\right)$ is endowed with an action of $G_{M}$, we get the claimed action of $G_{M}$.

Frobenius: The Frobenius on $\mathrm{H}^{i}\left(\mathfrak{X}_{\bar{K}}, \mathbb{L} \otimes_{\mathbb{Z}_{p}} \mathbb{B}_{\text {cris }}^{\nabla}\right)$ is induced by Frobenius on $\mathbb{B}_{\text {cris }}^{\nabla}$. Frobenius on $\mathrm{H}_{\mathrm{dR}}^{i}\left(X^{\mathrm{et}}, \mathbb{D}_{\text {cris }}^{\text {geo }}(\mathbb{L})\right)$ is constructed as follows. Fix a covering of $X$ by small affines $\mathcal{U}_{i}$ for $i \in I$, and for each of them choose parameters $T_{i, 1}, \ldots, T_{i, d} \in R_{\mathcal{U}_{i}}^{*}$. This choice provides a lift of Frobenius $F_{i}$ on each $\mathcal{U}_{i}$ as the unique $\mathcal{O}_{K}$-linear map sending $T_{i, j} \mapsto T_{i, j}^{p}$. Fix a total ordering on $I$. For every non-empty subset $J \subset I$ put $\mathcal{U}_{J}:=\prod_{i \in J} \mathcal{U}_{i}$ as formal schemes over $\mathcal{O}_{K}$ and $\mathcal{U}^{J}:=\cap_{i \in J} \mathcal{U}_{i}$. Note that $\mathcal{U}^{J} \subset \mathcal{U}_{J}$ is closed in an open $\mathcal{U}_{J}^{o} \subset \mathcal{U}_{J}$. Let $\mathcal{U}_{J}^{\text {DP }}$ be the $p$-adic completion of the $\mathbb{W}(k)$-divided power envelope of $\mathcal{U}_{J}^{o}$ with respect to the ideal defining $\mathcal{U}_{k}^{J}$. Let $F_{J}:=\prod_{i \in J} F_{i}: \mathcal{U}_{J} \rightarrow \mathcal{U}_{J}$. It induces a morphism $F_{J}: \mathcal{U}_{J}^{\mathrm{DP}} \rightarrow \mathcal{U}_{J}^{\mathrm{DP}}$. Note that $\mathcal{U}_{J}^{\mathrm{DP}}$ and $F_{J}$ define a complex for varying $J$.

Put $\mathbb{D}(\mathbb{L})_{J}:=\left.\mathbb{D}(\mathbb{L})\right|_{\mathcal{U}_{J}^{\text {PD }}}$ and let $\Phi_{J}: \mathbb{D}(\mathbb{L})_{J} \rightarrow \mathbb{D}(\mathbb{L})_{J}$ be the $F_{J}$-linear morphism defined by the non-degenerate Frobenius morphism $\Phi$ on $\mathbb{D}(\mathbb{L})$. For varying $J$ we get a morphism of double complexes

$$
\Phi_{\bullet, *}: \mathbb{D}(\mathbb{L}) \bullet \otimes \Omega_{\mathcal{U}_{\bullet}, \mathcal{O}_{K}}^{*} \widehat{\otimes} A_{\text {cris }} \rightarrow \mathbb{D}(\mathbb{L}) \bullet \otimes \Omega_{\mathcal{U}_{\bullet}, \mathcal{O}_{K}}^{*} \widehat{\otimes} A_{\text {cris }}
$$


We have natural morphisms $\mathcal{U}^{J} \rightarrow \mathcal{U}_{J}^{\mathrm{DP}}$ of PD thickenings of $\mathcal{U}_{k}^{J}$ which are compatible for varying $J$. It follows from the crystalline Poincaré lemma [BO, Th. 6.14] that the induced morphism

$$
\left.\mathbb{D}(\mathbb{L})_{J} \otimes \Omega_{\mathcal{U}_{J}, \mathcal{O}_{K}}^{*} \longrightarrow \mathbb{D}(\mathbb{L})\right|_{\mathcal{U}^{J}} \otimes \Omega_{\mathcal{U}^{J}, \mathcal{O}_{K}}^{*}
$$

is a quasi isomorphism. Thus the cohomology of $\mathbb{D}(\mathbb{L}) \bullet \otimes \Omega_{\mathcal{U}_{\bullet}, \mathcal{O}_{K}}^{*} \widehat{\otimes} B_{\text {cris }}$ is the cohomology of the

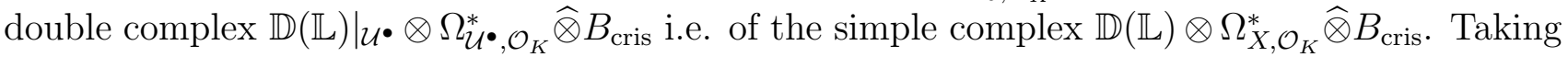
cohomology and using this identification, we get from $\Phi_{\bullet, *}$ the Frobenius map

$$
\varphi: \mathrm{H}^{i}\left(\mathrm{Sh}\left(X^{\mathrm{et}}\right)^{\mathbb{N}}, \mathbb{D}(\mathbb{L}) \otimes \Omega_{X, \mathcal{O}_{K}}^{\bullet} \widehat{\otimes} B_{\text {cris }}\right) \longrightarrow \mathrm{H}^{i}\left(\operatorname{Sh}\left(X^{\mathrm{et}}\right)^{\mathbb{N}}, \mathbb{D}(\mathbb{L}) \otimes \Omega_{X, \mathcal{O}_{K}}^{\bullet} \widehat{\otimes} B_{\text {cris }}\right) .
$$

Since $\mathbb{D}_{\text {cris }}^{\text {geo }}(\mathbb{L}) \cong \mathbb{D}(\mathbb{L}) \widehat{\otimes} B_{\text {cris }}$ we get the claimed Frobenius on $H_{\mathrm{dR}}^{i}\left(X^{\mathrm{et}}, \mathbb{D}_{\text {cris }}^{\text {geo }}(\mathbb{L})\right)$.

Remark 3.16. By construction $\mathrm{H}^{i}\left(\mathfrak{X}_{\bar{K}}, \mathbb{L} \otimes \mathbb{A}_{\text {cris }, \overline{\mathrm{K}}}^{\nabla}(m)\right) \cong \mathrm{H}^{i}\left(\mathfrak{X}_{\bar{K}}, \mathbb{L} \otimes \mathbb{A}_{\text {cris }, \overline{\mathrm{K}}}^{\nabla}\right)$ as $A_{\text {cris }}-$ modules and for $n \leq m$ using this identification the natural morphism $\mathrm{H}^{i}\left(\mathfrak{X}_{\bar{K}}, \mathbb{L} \otimes \mathbb{A}_{\text {cris, } \overline{\mathrm{K}}}^{\nabla}(m)\right) \rightarrow$ $\mathrm{H}^{i}\left(\mathfrak{X}_{\bar{K}}, \mathbb{L} \otimes \mathbb{A}_{\text {cris }, \overline{\mathrm{K}}}^{\nabla}(n)\right)$ is simply multiplication by $t^{m-n}$ on $\mathrm{H}^{i}\left(\mathfrak{X}_{\bar{K}}, \mathbb{L} \otimes \mathbb{A}_{\text {cris }, \overline{\mathrm{K}}}^{\nabla}\right)$. Thus

$$
\mathrm{H}^{i}\left(\mathfrak{X}_{\bar{K}}, \mathbb{L} \otimes \mathbb{B}_{\text {cris }, \overline{\mathrm{K}}}^{\nabla}(m)\right)=\mathrm{H}^{i}\left(\mathfrak{X}_{\bar{K}}, \mathbb{L} \otimes \mathbb{A}_{\text {cris }, \overline{\mathrm{K}}}^{\nabla}\right) \otimes_{A_{\text {cris }}} B_{\text {cris }}
$$

In particular we can replace the expression on the right in the statements of theorem 3.15 ,

We show first how to calculate explicitly the sheaves $\mathrm{R}^{j} v_{\bar{K}, *}^{\text {cont }}\left(\mathbb{L} \otimes_{\mathbb{Z}_{p}} \mathbb{B}_{\text {cris, }, \bar{K}}\right)$ for a $\mathbb{Q}_{p}$-adic étale sheaf $\mathbb{L}$ over $X \frac{\mathrm{et}}{K}$ according to the conventions of $\$ 2.8$.

Computation of $\mathrm{R}^{j} v_{M, *}^{\text {cont }}$ via continuous Galois cohomology. Consider an inverse system of sheaves $\mathcal{F}=\left\{\mathcal{F}_{n}\right\}_{n} \in \operatorname{Sh}\left(\mathfrak{X}_{M}\right)^{\mathbb{N}}$. Then for every $\mathcal{U}$ connected and étale affine over $X$ consider the inverse system $\left\{\mathcal{F}_{n}\left(\bar{R}_{\mathcal{U}}\right)\right\}_{n} \in \operatorname{Rep}\left(\mathcal{G}_{\mathcal{U}_{M}}\right)^{\mathbb{N}}$. Recall that we have defined the localization $\mathcal{F}\left(\bar{R}_{\mathcal{U}}\right):=\lim _{\infty \leftarrow n} \mathcal{F}_{n}\left(\bar{R}_{\mathcal{U}}\right)$. Given an abelian category $\mathcal{A}$ in [AI, §5.1] general results for the category of inverse systems $\mathcal{A}^{\mathbb{N}}$ are recalled. In particular if $\mathcal{A}$ admits enough injectives then also $\mathcal{A}^{\mathbb{N}}$ has enough injectives. For example one can derive the functor associating to an inverse systems of discrete $\mathcal{G}_{\mathcal{U}_{M}}$-modules $G:=\left\{G_{n}\right\}_{n}$ the abelian group $\left(\lim _{\infty \leftarrow n} G_{n}\right){ }^{\mathcal{G}_{\mathcal{U}_{M}}}$ and we get a $\delta$-functor $\mathrm{H}^{*}\left(\mathcal{G}_{\mathcal{U}_{M}}, G\right)$. If the system $G$ is Mittag-Leffler this is shown to coincide with the usual continuous group cohomology. In particular given $\mathcal{F}=\left\{\mathcal{F}_{n}\right\}_{n} \in \operatorname{Sh}\left(\mathfrak{X}_{M}\right)^{\mathbb{N}}$ we can define $\mathrm{H}_{\text {Gal }}^{i}(\mathcal{F})$ to be the sheaf associated to the contravariant functor sending $\mathcal{U}$, a connected and étale affine over $X$ to $\mathrm{H}^{i}\left(\mathcal{G}_{\mathcal{U}_{M}},\left\{\mathcal{F}_{n}\left(\bar{R}_{\mathcal{U}}\right)\right\}_{n}\right)$. We also have the sheaf $\mathrm{R}^{n} v_{M, *}(\mathcal{F})$ on $X^{\text {et }}$ obtained by deriving the functor $\mathcal{F} \mapsto \lim _{\infty \leftarrow n} v_{X, M, *} \mathcal{F}_{n}$. Then we have.

Lemma 3.17. For every $n \in \mathbb{N}$ there is a functorial homomorphism of sheaves

$$
f_{n}(\mathcal{F}): \mathrm{H}_{\text {Gal }}^{n}(\mathcal{F}) \longrightarrow \mathrm{R}^{n} v_{M, *}(\mathcal{F}) .
$$

Proof. This is a variant of [Err, Lemma 3.5] which is stated and proven for sheaves on $\mathfrak{X}_{M}$. We provide the main ingredients. Let $\mathfrak{U}_{M}$ be Faltings's site associated to $\mathcal{U}$. We then have a morphism of sites $j_{\mathcal{U}}: \mathfrak{X}_{M} \rightarrow \mathfrak{U}_{M}$ sending $(\mathcal{V}, W) \mapsto\left(\mathcal{V} \times_{\underline{X}} \mathcal{U}, W \times_{X} \mathcal{U}\right)$. It induces a morphism $j_{\mathcal{U}}^{*}: \operatorname{Sh}\left(\mathfrak{X}_{M}\right)^{\mathbb{N}} \longrightarrow \operatorname{Sh}\left(\mathfrak{U}_{M}\right)^{\mathbb{N}}$ which admits an exact left adjoint $j_{\mathcal{U}, !}$ given componentwise by extension by zero. In particular one deduces from this as in [Err, Lemma 3.5] that the 
sheaf $T^{n}(\mathcal{F})$ associated to the pre-sheaf $\mathcal{U} \mapsto \mathrm{H}^{n}\left(\mathfrak{U}_{M}, j_{\mathcal{U}}^{*}(\mathcal{F})\right)$ is a universal $\delta$-functor. Since $\mathrm{H}^{0}\left(\mathfrak{U}_{M}, j_{\mathcal{U}}^{*}(\mathcal{F})\right)=\mathrm{H}^{0}\left(\mathcal{U}, v_{M, *}(\mathcal{F})\right)$, we conclude that $T^{n}(\mathcal{F}) \cong \mathrm{R}^{n} v_{M, *}(\mathcal{F})$.

Note that $\mathrm{H}^{n}\left(\mathfrak{U}_{M},-\right)$ is the composite of the localization functor $\mathcal{F} \mapsto\left\{\mathcal{F}_{n}\left(\bar{R}_{\mathcal{U}}\right)\right\}_{n}$ with $\mathrm{H}^{0}\left(\mathcal{G}_{\mathcal{U}_{M}},{ }_{-}\right)$. The induced spectral sequence provides $\mathrm{H}^{0}\left(\mathcal{G}_{\mathcal{U}_{M}},\left\{\mathcal{F}_{n}\left(\bar{R}_{\mathcal{U}}\right)\right\}_{n}\right) \longrightarrow \mathrm{H}^{n}\left(\mathfrak{U}_{M}, j_{\mathcal{U}}^{*}(\mathcal{F})\right)$. Composing this with the morphism to $T^{n}(\mathcal{F})(\mathcal{U}) \cong \mathrm{R}^{n} v_{M, *}(\mathcal{F})(\mathcal{U})$ we get the claimed map.

In [Err, Lemma 3.5] it is shown that if we work with sheaves on $\mathfrak{X}_{M}$, not with continuous sheaves, the above map is an isomorphism. This is not true in the general context of continuous sheaves. Assume that $M=\bar{K}$ then we have the following:

Proposition 3.18. For every $n \in \mathbb{N}$ the morphism $f_{n}(\mathcal{F})$ has kernel and cokernel annihilated by any element of $\mathbb{I}^{2 i}$ where $\mathbb{I}$ is the ideal introduced in \$1.2 in the following cases: (a) $\mathcal{F}:=\left\{\mathbb{W}_{n, \bar{K}}\right\}_{n} ;(b) \mathcal{F}:=\left\{\mathcal{O}_{\mathfrak{X}_{M}} / p^{n} \mathcal{O}_{\mathfrak{X}_{M}}\right\}_{n} ;(c) \mathcal{F}=\mathbb{A}_{\text {cris }, \bar{K}}(m)$ for every $m \in \mathbb{Z} ; \quad$ (d) $\mathcal{F}=$ $\operatorname{Gr}^{r} \mathbb{A}_{\text {cris }, \overline{\mathrm{K}}}(m)$ for every $m$ and $r \in \mathbb{Z}$.

Proof. The proposition follows from [AI, Thm. 6.12] after minor changes if the assumptions of loc. cit. are satisfied. The statement in loc. cit. provides an isomorphism after inverting $[\varepsilon]-1$ and working with the pointed site $\mathfrak{X}_{\bar{K}}^{\bullet}$. An inspection of the proof gives our claim. First of all the proof works for $\mathfrak{X}_{\bar{K}}$ and not only for $\mathfrak{X}_{\bar{K}}^{\bullet}$ using [Err, Lemma 3.5] which is the analogue of [AI, Prop 4.4] for $\mathfrak{X}_{\bar{K}}$ instead of $\mathfrak{X}_{\bar{K}}^{\bullet}$. Secondly the two cohomology groups are related by two spectral sequences, one in [AI, Formula (19)] and the other in [AI, Prop. 6.15] and the proofs of [AI, lemma $6.13 \&$ Prop. 6.15] show that each degenerates if we multiply by any element of $\mathbb{I}^{i}$ and not only after inverting $[\varepsilon]-1$.

We now verify that the assumptions hold. Assumptions (i) and (ii) state the existence of enough small affines of $X$. The fact that the other Assumptions hold for $\left\{\mathbb{W}_{n, \bar{K}}\right\}_{n}$ and for $\mathcal{F}:=\left\{\mathcal{O}_{\mathfrak{X}_{M}} / p^{n} \mathcal{O}_{\mathfrak{X}_{M}}\right\}_{n}$ is precisely the content of [AI, Thm. 6.16(A)\&(B)]. We pass to $\mathbb{A}_{\text {cris }, \overline{\mathrm{K}}}(m)$. Assumptions (iii)-(vi) concern the behavior of the sheaves $\mathcal{F}_{n}$ restricted to the subsite $\mathfrak{U}_{\bar{K}, n}$ of $\mathfrak{X}_{\bar{K}}$ for $n \gg 0$ for every small $\mathcal{U}$ of $X^{\text {et }}$; see2.6 for the definition of $\mathfrak{U}_{\bar{K}, n}$. Since $\left.\mathbb{A}_{\text {cris,n, } \overline{\mathrm{K}}}^{\prime}(m)\right|_{\mathfrak{U}_{\bar{K}, n}} \cong$ $\left.\mathbb{A}_{\text {cris,n, } \overline{\mathrm{K}}}^{\prime}(m)\right|_{\mathfrak{U}_{\bar{K}, n}}\left\langle X_{1}, \ldots, X_{d}\right\rangle$ by lemma 2.33 this is a sheaf of free $\left.\mathbb{A}_{\text {cris,n, } \overline{\mathrm{K}}}^{\prime}(m)\right|_{\mathfrak{U}_{\bar{K}, n}}$-modules. On the other hand $\mathbb{A}_{\text {cris }, \mathrm{n}, \overline{\mathrm{K}}}^{\prime}(m) \cong A_{\text {cris }} / p^{n} A_{\text {cris }} \otimes \mathbb{W}_{n, \bar{K}}$ by lemma 2.26 . We conclude since assumptions (iii)-(vi) hold for the continuous sheaf $\left\{\mathbb{W}_{n, \bar{K}}\right\}_{n}$.

The statement concerning $\operatorname{Gr}^{r} \mathbb{A}_{\text {cris }, \bar{K}}(m)$ is proven similarly. We have

$$
\left.\left.\mathrm{Fil}^{r} \mathbb{A}_{\text {cris }, \mathrm{n}, \overline{\mathrm{K}}}^{\prime}(m)\right|_{\mathfrak{U}_{\bar{K}, n}} \cong \sum_{s_{0}+\ldots+s_{d} \geq r-m} \operatorname{Fil}^{s_{0}}\left(A_{\text {cris }} / p^{n} A_{\text {cris }}\right) \otimes \mathbb{W}_{n, \bar{K}}\right|_{\mathfrak{U}_{\bar{K}, n}} X_{1}^{\left[s_{1}\right]} \cdots X_{d}^{\left[s_{d}\right]}
$$

with $X_{j}:=1 \otimes T_{j}-\widetilde{T}_{j} \otimes 1$. In particular

$$
\left.\left.\mathrm{Gr}^{r} \mathbb{A}_{\text {cris,n, }, \overline{\mathrm{K}}}^{\prime}\right|_{\mathfrak{U}_{\bar{K}, n}} \cong \oplus_{s_{0}+\ldots+s_{d}=r-m}\left(\mathcal{O}_{\mathfrak{X}_{M}} / p^{n} \mathcal{O}_{\mathfrak{X}_{M}}\right)\right|_{\mathfrak{U}_{\bar{K}, n}} \xi^{\left[s_{0}\right]} \cdot X_{1}^{\left[s_{1}\right]} \cdots X_{d}^{\left[s_{d}\right]}
$$

is a free $\mathcal{O}_{\mathfrak{X}_{M}} /\left.p^{n} \mathcal{O}_{\mathfrak{X}_{M}}\right|_{\mathfrak{U}_{\bar{K}, n}}$-module. Since the Assumptions of loc cit. for the inverse system $\left\{\mathcal{O}_{\mathfrak{X}_{M}} / p^{n} \mathcal{O}_{\mathfrak{X}_{M}}\right\}_{n}$ are satisfied we are done.

Denote by $\mathcal{H}^{i}(m)$ (resp. $\mathcal{H}^{i}\left(\mathrm{Fil}^{r}, m\right)$, resp. $\left.\mathcal{H}^{i}\left(\mathrm{Gr}^{r}, m\right)\right)$ the sheaf associated to the contravariant functor which associates to a small affine open $\mathcal{U}=\operatorname{Spf}\left(R_{\mathcal{U}}\right)$ of $X^{\text {et }}$ the value

$\mathrm{H}^{i}\left(\mathcal{G}_{\mathcal{U}, \bar{K}}, A_{\text {cris }, \overline{\mathrm{K}}}(m)\left(\bar{R}_{\mathcal{U}}\right)\right)$, resp. $\mathrm{H}^{i}\left(\mathcal{G}_{\mathcal{U}, \bar{K}}, \mathrm{Fil}^{r} A_{\text {cris }, \overline{\mathrm{K}}}(m)\left(\bar{R}_{\mathcal{U}}\right)\right)$, resp. $\mathrm{H}^{i}\left(\mathcal{G}_{\mathcal{U}, \bar{K}}, \operatorname{Gr}^{r} A_{\text {cris }, \overline{\mathrm{K}}}(m)\left(\bar{R}_{\mathcal{U}}\right)\right)$.

The cohomology considered is the continuous Galois cohomology. Then we have. 
Lemma 3.19. For every $r \in \mathbb{N}$ and $m \in \mathbb{Z}$ we have morphisms $\mathcal{H}^{i}(m) \longrightarrow \mathrm{H}_{\text {Gal }}^{i}\left(\mathbb{A}_{\text {cris }, \overline{\mathrm{K}}}(m)\right)$, $\mathcal{H}^{i}\left(\mathrm{Gr}^{r}, m\right) \longrightarrow \mathrm{H}_{\text {Gal }}^{i}\left(\operatorname{Gr}^{r} \mathbb{A}_{\text {cris }, \overline{\mathrm{K}}}(m)\right)$ and $\mathcal{H}^{i}\left(\mathrm{Fil}^{r}, m\right) \longrightarrow \mathrm{H}_{\text {Gal }}^{i}\left(\right.$ Fil $\left.^{r} \mathbb{A}_{\text {cris }, \overline{\mathrm{K}}}(m)\right)$ which are compatible with the maps induced by the projection $\mathrm{Fil}^{r} \rightarrow \mathrm{Gr}^{r}$ and have kernel and cokernel annihilated by $\mathbb{I}$.

Proof. It follows from lemma 2.29 and lemma 2.35 that the inverse system of $\mathcal{G}_{\mathcal{U}, M}$-modules $A_{\text {cris, }, \overline{\mathrm{K}}}^{\prime}(m)\left(\bar{R}_{\mathcal{U}}\right)$ is contained in the localization $\left.\mathbb{A}_{\text {cris, }, \mathrm{n}, \overline{\mathrm{K}}}^{\prime}(m)\right)\left(\bar{R}_{\mathcal{U}}\right)$ for every small affine $\mathcal{U}$ of $X$ with cokernel annihilated by any element of $\mathbb{I}$. This provides the first morphism and the claim regarding its kernel and cokernel. Similarly using the description of $\operatorname{Gr}^{r} \mathbb{A}_{\text {cris }, \overline{\mathrm{K}}}(m)$ given in the proof of 3.18, we deduce that $\operatorname{Gr}^{r} A_{\text {cris,n, }, \overline{\mathrm{K}}}\left(\bar{R}_{\mathcal{U}}\right)$ is contained in $\operatorname{Gr}^{r} \mathbb{A}_{\text {cris,n, }, \overline{\mathrm{K}}}\left(\bar{R}_{\mathcal{U}}\right)$ with cokernel annihilated by $\mathbb{I}$. This provides the second map and the subsequent statement. Using induction on $r$ and the fact that $\mathrm{Gr}^{r}=\mathrm{Fil}^{r} / \mathrm{Fil}^{r+1}$ we deduce that also $\mathrm{Fil}^{r} A_{\text {cris, }, \mathrm{n}, \overline{\mathrm{K}}}\left(\bar{R}_{\mathcal{U}}\right)$ is contained in Fil $^{r} \mathbb{A}_{\text {cris, }, \mathrm{K}}\left(\bar{R}_{\mathcal{U}}\right)$ with cokernel annihilated by $\mathbb{I}$. This gives the last morphism and proves the assertion concerning its kernel and cokernel.

Corollary 3.20. Let $\mathcal{M}$ be a coherent $\mathcal{O}_{X_{K}}$-module such that for every small affine $\mathcal{U}$ the $R_{\mathcal{U}} \otimes_{\mathcal{O}_{K}} K$-module $\mathcal{M}(\mathcal{U}) \otimes_{\mathcal{O}_{K}} K$ is projective. We view $\mathcal{M}$ as a sheaf on $\mathfrak{X}_{\bar{K}}$ via $v_{\bar{K}}^{*}$. For every $r \in \mathbb{Z} \cup\{-\infty\}$ we have:

$$
\mathrm{R}^{j} v_{\bar{K}, *}^{\text {cont }}\left(\text { Fil }^{r} \mathbb{B}_{\text {cris }, \bar{K}} \otimes_{\mathcal{O}_{X}} \mathcal{M}\right)= \begin{cases}0 & \text { if } j \geq 1 \\ \text { Fil }^{r} B_{\text {cris }} \widehat{\otimes} \mathcal{M} & \text { if } j=0\end{cases}
$$

Proof. Note the we have a natural map Fil ${ }^{r} B_{\text {cris }} \widehat{\otimes} \mathcal{M} \longrightarrow v \overline{\mathrm{cont}}^{\mathrm{u}, *}\left(\mathrm{Fil}^{r} \mathbb{B}_{\text {cris, }, \overline{\mathrm{K}}} \otimes_{\mathcal{O}_{\mathcal{X}_{M}}^{\text {un }}} \mathcal{M}\right)$. Thus both statements are local on $X$. We may then assume that $X=\mathcal{U}$ is a small affine so that $\mathcal{M} \otimes_{\mathcal{O}_{K}} K$ is a direct summand in a free $\mathcal{O}_{X_{K}}$-module. Since $\mathrm{R}^{j} v \frac{\text { cont }}{\bar{K}, *}$ commutes with direct sums we may assume that $\mathcal{M} \otimes_{\mathcal{O}_{K}} K$ is a free module and we are reduced to prove the corollary in the case that $\mathcal{M}=\mathcal{O}_{X}$. Fix integers $m$ and $r \in \mathbb{Z}$ with $m \leq N$. We start by considering the following statements:

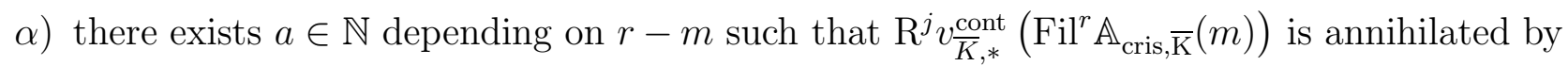
$t^{a}$ if $j \geq 1$.

$\beta)$ there exists $c \in \mathbb{N}$, depending on $a$, such that the kernel of $\mathrm{R}^{j} v_{\bar{K}, *}^{\text {cont }}\left(\right.$ Fil $\left.^{r} \mathbb{A}_{\text {cris }, \overline{\mathrm{K}}}(m-a)\right) \longrightarrow$ $\mathrm{R}^{j} v \frac{\text { cont }}{K, *}\left(\mathrm{Fil}^{r-a} \mathbb{A}_{\text {cris }, \overline{\mathrm{K}}}(m-a)\right)$ is annihilated by $p^{c}$ for every $j \geq 1$.

$\gamma$ ) the map $\mathcal{M} \widehat{\otimes} \mathrm{Fil}^{r} A_{\text {cris }} \rightarrow v \bar{K}, *_{\text {cont }}\left(\operatorname{Fil}^{r} \mathbb{A}_{\text {cris }, \overline{\mathrm{K}}} \otimes_{\mathcal{O}_{\mathfrak{X}_{M}}^{\text {un }}} \mathcal{M}\right)$ is an isomorphism for all $r$.

First of all we remark that these statements imply the corollary in the case that $\mathcal{M}=\mathcal{O}_{X}$. Indeed together with 3.1 these claims imply all the statements of the corollary except for the vanishing of $\mathrm{R}^{j} v \frac{\text { cont }}{\bar{K}, *}\left(\mathbb{L} \otimes_{\mathbb{Z}_{p}} F^{r}{ }^{r} \mathbb{B}_{\text {cris }, \overline{\mathrm{K}}} \otimes_{\mathcal{O}_{X}} \mathcal{M}\right)$ for $r \in \mathbb{Z}$ and $j \geq 1$. Note that the image of the continuous sheaf $\mathrm{Fil}^{r} \mathbb{A}_{\text {cris }, \overline{\mathrm{K}}}(m)$ in $\mathrm{Fil}^{r-a} \mathbb{A}_{\text {cris }, \overline{\mathrm{K}}}(m-a)$ is $t^{a} \cdot \mathrm{Fil}^{r-a} \mathbb{A}_{\text {cris }, \overline{\mathrm{K}}}(m-a)$. It follows from $(\alpha)$ that the map

$$
\mathrm{R}^{j} v_{\bar{K}, *}\left(\operatorname{Fil}^{r} \mathbb{A}_{\text {cris }, \overline{\mathrm{K}}}(m)\right) \rightarrow \mathrm{R}^{j} v_{\bar{K}, *}\left(\mathrm{Fil}^{r} \mathbb{A}_{\text {cris }, \overline{\mathrm{K}}}(m-a)\right)
$$


factors via the kernel of the map

$$
\mathrm{R}^{j} v_{\bar{K}, *}\left(\mathrm{Fil}^{r} \mathbb{A}_{\text {cris }, \overline{\mathrm{K}}}(m)\right) \longrightarrow \mathrm{R}^{j} v_{\bar{K}, *}\left(\mathrm{Fil}^{r-a} \mathbb{A}_{\text {cris }, \overline{\mathrm{K}}}(m-a)\right)
$$

which is annihilated by $p^{c}$ by $(\beta)$. Since multiplication by $p$ is an isomorphism on $\mathrm{Fil}^{r} \mathbb{B}_{\text {cris,M }}$ by 2.41, also the vanishing of $\mathrm{R}^{j} v \frac{\text { cont }}{\bar{K}, *}\left(\mathrm{Fil}^{r} \mathbb{B}_{\text {cris, }, \overline{\mathrm{K}}} \mathcal{M}\right)$ for $r \in \mathbb{Z}$ and $j \geq 1$ follows.

Now we start proving the statements $\alpha), \beta), \gamma)$. In view of 2.36 to prove statement $(\gamma)$ we need to prove that for every small affine the map $R_{\mathcal{U}} \widehat{\otimes} \mathrm{Fil}^{r} A_{\text {cris }} \rightarrow \mathrm{Fil}^{r} A_{\text {cris, }, \overline{\mathrm{K}}}\left(\bar{R}_{\mathcal{U}}\right)^{\mathcal{G}_{\mathcal{U}, \bar{K}}}$ is an isomorphism. This follows from [AB, Prop. 41].

Recall that $\mathrm{Fil}^{r} \mathbb{A}_{\text {cris }, \overline{\mathrm{K}}}(m) \cong \mathrm{Fil}^{r-m} \mathbb{A}_{\text {cris }, \overline{\mathrm{K}}}$ as continuous sheaves on $\mathfrak{X}_{\bar{K}}$. Thus, we may also assume that $m=0$. Given $r \in \mathbb{N}$, since $t^{r} \in \mathrm{Fil}^{r} A_{\text {cris }}$, the cokernel of the inclusion $\mathrm{Fil}^{r} \mathbb{A}_{\text {cris }, \overline{\mathrm{K}}} \subset \mathbb{A}_{\text {cris }, \overline{\mathrm{K}}}$ is annihilated by $t^{r}$. Hence it suffices to prove $(\alpha)$ for $r=0$. Recall from 1.2 that $t \in \mathbb{I}$ since $t=(1-[\varepsilon]) u$ with $u$ a unit in $A_{\text {cris }}$ by [Fo, $\left.\S 5.2 .4 \& \S 5.2 .8(\mathrm{ii})\right]$. Then claim $(\alpha)$ follows from proposition [3.18, lemma 3.19 and the fact that $\mathrm{H}^{i}\left(\mathcal{G}_{\mathcal{U}, \bar{K}}, A_{\text {cris, } \overline{\mathrm{K}}}\left(\bar{R}_{\mathcal{U}}\right)(m)\right)$ is annihilated by $(1-[\varepsilon])^{2(d+1)} \mathbb{I}^{2}$ proven in corollary [AB, Cor. 24]. Here $d$ is the relative dimension of $X$ over $\mathcal{O}_{K}$.

We are left to show $(\beta)$. Proceeding inductively on $a$ it suffices to show that for every $r \in \mathbb{Z}$ and every $n \in \mathbb{N}$ the cokernel of the map $\mathrm{R}^{j-1} v_{\bar{K}, *}^{\text {cont }}\left(\right.$ Fil $\left.^{r} \mathbb{A}_{\text {cris }, \overline{\mathrm{K}}}^{\prime}\right) \rightarrow \mathrm{R}^{j-1} v \frac{\text { cont }}{\bar{K}, *}\left(\operatorname{Gr}^{r} \mathbb{A}_{\text {cris }, \overline{\mathrm{K}}}^{\prime}\right)$ is annihilated by a power of $p$ (independent of $n$ ). Consider the commutative diagram

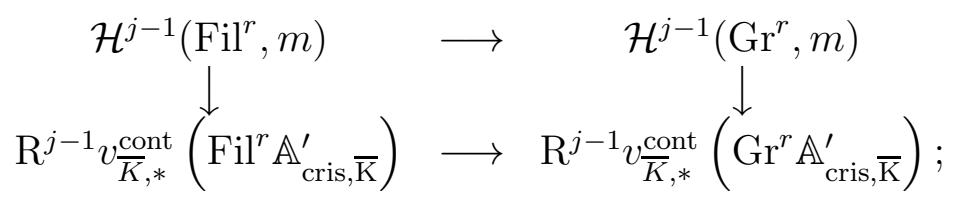

obtained from 3.18 and lemma 3.19, For every small affine $\mathcal{U}$, the map

$$
\mathrm{H}^{j-1}\left(\mathcal{G}_{\mathcal{U}, \bar{K}}, \operatorname{Fil}^{r} A_{\text {cris }, \overline{\mathrm{K}}}(m)\left(\bar{R}_{\mathcal{U}}\right)\right) \longrightarrow \mathrm{H}^{j-1}\left(\mathcal{G}_{\mathcal{U}, \bar{K}}, \operatorname{Gr}^{r} A_{\text {cris }, \overline{\mathrm{K}}}(m)\left(\bar{R}_{\mathcal{U}}\right)\right)
$$

has cokernel annihilated by a power of $p$ by [AB, Pf. Lemme 36]. This also applies to the associated sheaves i.e., to the map $\mathcal{H}^{j-1}\left(\mathrm{Fil}^{r}, m\right) \longrightarrow \mathcal{H}^{j-1}\left(\mathrm{Gr}^{r}, m\right)$. The right vertical morphism in the diagram has kernel and cokernel annihilated by $\mathbb{I}^{2(j-1)+1}$ by proposition 3.18 and lemma 3.19. We conclude that the same applies to the cokernel of the lower horizontal arrow. The conclusion follows.

Proof. (of theorem 3.15) Thanks to lemma 2.41, if $\mathbb{L}$ is a $p$-adic sheaf sheaf, the sequence

$$
\begin{aligned}
(*) 0 \longrightarrow \mathbb{L} \otimes_{\mathbb{Z}_{p}} \mathrm{Fil}^{r} \mathbb{B}_{\text {cris }, \overline{\mathrm{K}}}^{\nabla} \longrightarrow \mathbb{L} \otimes_{\mathbb{Z}_{p}} \mathrm{Fil}^{r} \mathbb{B}_{\text {cris }, \overline{\mathrm{K}}} \stackrel{\nabla}{\longrightarrow} \mathbb{L} \otimes_{\mathbb{Z}_{p}} \mathrm{Fil}^{r-1} \mathbb{B}_{\text {cris }, \overline{\mathrm{K}}} \otimes_{\mathcal{O}_{X}} \Omega_{X / \mathcal{O}_{K}}^{1} \stackrel{\nabla}{\longrightarrow} \cdots \\
\cdots \stackrel{\nabla}{\longrightarrow} \mathbb{L} \otimes_{\mathbb{Z}_{p}} \mathrm{Fil}^{r-d} \mathbb{B}_{\text {cris }, \overline{\mathrm{K}}} \otimes_{\mathcal{O}_{X}} \Omega_{X / \mathcal{O}_{K}}^{d} \longrightarrow 0
\end{aligned}
$$

is exact for every $r \in \mathbb{Z}$. Due to 3.9 the complex $\mathbb{L} \otimes_{\mathbb{Z}_{p}} \mathrm{Fil}^{r-\bullet} \mathbb{B}_{\text {cris, } \overline{\mathrm{K}}} \otimes_{\mathcal{O}_{X}} \Omega_{X / \mathcal{O}_{K}}^{\bullet}$ is isomorphic to the complex Fil ${ }^{r-\bullet}\left(\mathbb{D}_{\text {cris }}^{\text {geo }}(\mathbb{L}) \otimes_{\mathcal{O}_{X} \otimes_{\mathcal{O}_{K}} A_{\text {cris }}} \mathbb{B}_{\text {cris }, \overline{\mathrm{K}}} \otimes_{\mathcal{O}_{X}} \Omega_{X / \mathcal{O}_{K}}^{\bullet}\right)$. This provides an isomorphism

$$
\mathrm{H}^{i}\left(\mathfrak{X}_{\bar{K}}, \mathbb{L} \otimes \mathrm{Fil}^{r} \mathbb{B}_{\text {cris }, \overline{\mathrm{K}}}^{\nabla}\right) \stackrel{\sim}{\longrightarrow} \mathbb{H}^{i}\left(\mathfrak{X} \overline{\bar{K}}, \mathrm{Fil}^{r-\bullet}\left(\mathbb{D}_{\text {cris }}^{\mathrm{geo}}(\mathbb{L}) \otimes_{\mathcal{O}_{X} \otimes_{\mathcal{O}_{K}} A_{\text {cris }}} \mathbb{B}_{\text {cris }, \overline{\mathrm{K}}} \otimes_{\mathcal{O}_{X}} \Omega_{X / \mathcal{O}_{K}}^{\bullet}\right)\right)
$$


Here we view $\mathrm{Fil}^{r-\bullet}\left(\mathbb{D}_{\text {cris }}^{\text {geo }}(\mathbb{L}) \otimes_{\mathcal{O}_{X} \otimes_{\mathcal{O}_{K}} A_{\text {cris }}} \mathbb{B}_{\text {cris }, \overline{\mathrm{K}}} \otimes_{\mathcal{O}_{X}} \Omega_{X / \mathcal{O}_{K}}\right)$ as the inductive system of complexes of continuous sheaves $\mathrm{Fil}^{r-\bullet}\left(\mathbb{D}_{\text {cris }}^{\text {geo }}(\mathbb{L}) \otimes_{\mathcal{O}_{X} \otimes_{\mathcal{O}_{K}} A_{\text {cris }}} \mathbb{A}_{\text {cris }, \bar{K}}(m) \otimes_{\mathcal{O}_{X}} \Omega_{X / \mathcal{O}_{K}}^{\bullet}\right)$ and we apply the construction of definition 2.8 to the continuous hyper-cohomology $\mathbb{H}^{*}\left(\mathfrak{X}_{\bar{K}},-\right)$ of these complexes. It follows from corollary 3.20 that $\mathrm{Fil}^{r-\bullet}\left(\mathbb{D}_{\text {cris }}^{\text {geo }}(\mathbb{L}) \otimes_{\mathcal{O}_{X} \otimes_{\mathcal{O}_{K}} A_{\text {cris }}} \mathbb{B}_{\text {cris }, \overline{\mathrm{K}}} \otimes_{\mathcal{O}_{X}} \Omega_{X / \mathcal{O}_{K}}^{\bullet}\right)$ is acyclic for $v_{\bar{K}, *}$ and that its image via $v_{\bar{K}, *}$ is $\mathrm{Fil}^{r-\bullet}\left(\mathbb{D}_{\text {cris }}^{\text {geo }}(\mathbb{L}) \otimes_{\mathcal{O}_{X}} \Omega_{X / \mathcal{O}_{K}}^{\bullet}\right)$. Thus, we have an isomorphism $\mathbb{H}^{i}\left(X^{\mathrm{et}}, \mathrm{Fil}^{\mathrm{r}-\bullet} \mathbb{D}_{\text {cris }}^{\text {geo }}(\mathbb{L}) \otimes_{\mathcal{O}_{X}} \Omega_{X / \mathcal{O}_{K}}^{\bullet} \stackrel{\sim}{\longrightarrow} \mathbb{H}^{i}\left(\mathfrak{X}_{\bar{K}}, \mathrm{Fil}^{r-\bullet}\left(\mathbb{D}_{\text {cris }}^{\text {geo }}(\mathbb{L}) \otimes_{\mathcal{O}_{X} \otimes_{\mathcal{O}_{K}} A_{\text {cris }}} \mathbb{B}_{\text {cris }, \overline{\mathrm{K}}} \otimes_{\mathcal{O}_{X}} \Omega_{X / \mathcal{O}_{K}}\right)\right)\right.$

Compatibility with $G_{M}$-action: These are isomorphisms of $G_{M}$-modules with $G_{M}$-structure given as explained after the theorem resolving $\mathbb{L} \otimes \mathrm{Fil}^{r} \mathbb{A}_{\text {cris }, \overline{\mathrm{K}}}^{\nabla}(m)$ (resp. $\mathbb{L} \otimes_{\mathbb{Z}_{p}} \mathrm{Fil}^{r-\bullet} \mathbb{A}_{\text {cris, } \overline{\mathrm{K}}}(m) \otimes_{\mathcal{O}_{X}}$ $\Omega_{X / \mathcal{O}_{K}}^{\bullet}$ ) with the pull-back via $\beta_{M, \bar{K}}^{*}$ of an injective resolution of $\mathbb{L} \otimes \mathrm{Fil}^{r} \mathbb{A}_{\text {cris,M }}^{\nabla}(m)$ (resp. of the complex $\mathbb{L} \otimes_{\mathbb{Z}_{p}} \mathrm{Fil}^{r-\bullet} \mathbb{A}_{\text {cris, }, \mathrm{M}}(m) \otimes_{\mathcal{O}_{X}} \Omega_{X / \mathcal{O}_{K}}^{\bullet}$.

Compatibility with Frobenius: Fix a covering of $X$ by small affines $\mathcal{U}_{i}$, for $i \in I$ and for each of them choose parameters $T_{i, 1}, \ldots, T_{i, d} \in R_{\mathcal{U}_{i}}^{*}$. For every subset $J \subset I$ let $\mathcal{U}^{J} \subset \mathcal{U}_{J}^{o} \subset \mathcal{U}_{J}$ be as in the notation introduced after theorem 3.15 . Let $\mathfrak{U}_{\bar{K}}^{J}$ be Faltings' site associated to $\mathcal{U}^{J}$ and consider the continuous morphism $j_{J}: \mathfrak{X}_{\bar{K}} \rightarrow \mathfrak{U}_{\bar{K}}^{J}$ sending $(\mathcal{V}, W)$ to $(\mathcal{V}, W) \times_{\left(X, X_{K}\right)}\left(\mathcal{U}^{J}, \mathcal{U}_{K}^{J}\right)$. The inverse image $j_{J}^{*}$ of a sheaf on $\mathfrak{X}_{\bar{K}}$ is the restriction of $\mathcal{F}$ to $\mathfrak{U}_{\bar{K}}^{J}$ viewed as a subcategory of $\mathfrak{X}_{\bar{K}}$.

Define $\mathbb{A}_{\text {cris }, \mathcal{U}_{J}, n}$ to be the $\mathbb{W}(k)$-DP sheaf of algebras in $\operatorname{Sh}\left(\mathfrak{U}_{\bar{K}}^{J}\right)$ of $\mathbb{A}_{\text {inf }, \mathcal{U}_{J}, n}$ with respect to the kernel of

$$
\mathbb{W}_{n}\left(\mathcal{O}_{\mathfrak{U}} \frac{J}{K} / p \mathcal{O}_{\mathfrak{U} \frac{J}{K}}\right) \longrightarrow \mathcal{O}_{\mathfrak{U}}{ }_{K}^{J} / p^{n} \mathcal{O}_{\mathfrak{U}} \frac{J}{K}
$$

defined by $\theta_{M}$. Its existence is proven as in 92.5 . Since $\mathcal{O}_{\mathfrak{U}_{K}^{J}}=\left.\mathcal{O}_{\mathfrak{X}_{\bar{K}}}\right|_{\mathfrak{L}_{K}^{J}}$ we have a natural morphism

$$
\mathbb{A}_{\text {cris }, \mathcal{U}_{J}, n}^{\nabla} \longrightarrow j_{J}^{*}\left(\mathbb{A}_{\text {cris }, X, n}^{\nabla}\right)
$$

and it follows from loc. cit. that such a morphism is an isomorphism. Define $\mathbb{A}_{\text {cris }, \mathcal{U}_{J}, n}$ as the

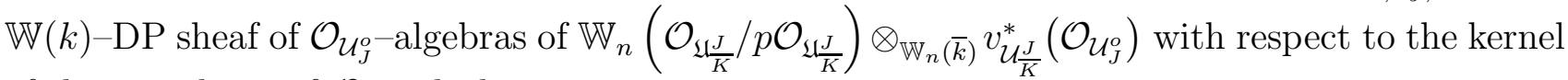
of the morphism of $\mathcal{O}_{\mathcal{U}_{J}^{o}}$-algebras

$$
\mathbb{A}_{\text {inf }, J, n}^{+} \otimes_{\mathbb{W}_{n}(\bar{k})} v_{\mathcal{U}_{K} \frac{J}{K}}^{*}\left(\mathcal{O}_{\mathcal{U}_{J}^{o}}\right) \longrightarrow \mathcal{O}_{\mathfrak{U} \frac{J}{K}} / p^{n} \mathcal{O}_{\mathfrak{U} \frac{J}{K}}
$$

defined by the $\mathcal{O}_{\mathcal{U}_{J}^{o-l i n e a r}}$ extension of $\theta_{M}$.

Lemma 3.21. The sheaf $\mathbb{A}_{\text {cris }, \mathcal{U}_{J}, n}$ exists and

$$
\left.\left.\mathbb{A}_{\text {cris }, \mathcal{U}_{J}, n}\right|_{\mathfrak{U}_{n}^{J}} \cong \mathbb{A}_{\text {cris }, \mathcal{U}^{J}, n}^{\nabla}\right|_{\mathfrak{U}_{n}^{J}}\left\langle X_{i, 1}, \ldots, X_{i, d}\right\rangle_{i \in J}
$$

where $X_{i, j}:=1 \otimes T_{i, j}-\widetilde{T}_{i, j} \otimes 1$ for every $i \in I$ and every $1 \leq j \leq d$. For every $h \in J$ one also has an isomorphism

$$
\mathbb{A}_{\text {cris }, \mathcal{U}_{J}, n} \cong j_{J}^{*}\left(\mathbb{A}_{\text {cris }, X, n}\right)\left\langle Y_{i, 1}^{(h)}, \ldots, Y_{i, d}^{(h)}\right\rangle_{i \in J, i \neq h}
$$

where $Y_{i, j}^{(h)}$, for $i \in J$ with $i \neq h$ and for $1 \leq j \leq d$ are regular elements generating the ideal defining the closed immersion $\mathcal{U}^{J} \subset\left(\mathcal{U}_{h} \times_{\mathcal{O}_{K}} \mathcal{U}_{i}\right)^{o}$. In particular $\mathbb{A}_{\text {cris }, \mathcal{U}_{J}, n}$ is a free $j_{J}^{*}\left(\mathbb{A}_{\text {cris }, X, n}\right)$ module. 
Proof. The existence of the sheaf and the formula for its restriction to $\mathfrak{U}_{n}^{J}$ is proven as in $\$ 2.6$, A similar argument implies the last formula over $\mathfrak{U}_{n}^{J}$. A descent argument allows to conclude that the formula holds also over $\mathfrak{U}^{J}$. The last statement follows from the properties of divided powers: a basis is given by monomials in the elements $\gamma_{j}\left(Y_{i, \ell}^{(h)}\right)$ for $j \in \mathbb{N}, 1 \leq \ell \leq d$ and $i \in J$ but $i \neq h$ taking $\gamma_{j}$ to be as in $\$ 1.2$, see $\$ 2.6$ for details.

Let $\mathbb{A}_{\text {cris }, J, n}^{\nabla}\left(\operatorname{resp} . \mathbb{A}_{\text {cris }, J, n}\right)$ be $j_{J, *}\left(\mathbb{A}_{\text {cris }, \mathcal{U}^{J}, n}^{\nabla}\right)\left(\operatorname{resp} . j_{J, *}\left(\mathbb{A}_{\text {cris }, \mathcal{U}^{J}, n}\right)\right)$. Define $\mathbb{A}_{\text {cris }, J}\left(\right.$ resp. $\left.\mathbb{A}_{\text {cris }, J}\right)$ as the system $\left\{\mathbb{A}_{\text {cris }, \mathrm{J}, n}^{\nabla}\right\}_{n}$ (resp. $\left\{\mathbb{A}_{\text {cris }, \mathrm{J}, n}\right\}_{n}$ ) and $\mathbb{B}_{\text {cris }, J}^{\nabla}$ (resp. $\mathbb{B}_{\text {cris }, J}$ ) for the inductive systems given by multiplication by $t$. We also write $\mathbb{B}_{\text {cris }, J} \otimes_{\mathcal{O}_{\mathcal{U}_{J}}} \Omega_{\mathcal{U}_{J} / \mathcal{O}_{K}}^{*}$ for the inductive system, with respect to multiplication by $t$, associated to the push-forward via $j_{J, *}$ of $\mathbb{A}_{\text {cris }, \mathcal{U}_{J}} \otimes_{\mathcal{O}_{\mathfrak{U}^{J}}^{\text {un }}} v_{\mathcal{U}^{J}, \bar{K}}^{*}\left(\Omega_{\mathcal{U}_{J} / \mathcal{O}_{K}}^{*}\right)$. We then get a long exact sequence of continuous sheaves

$$
0 \longrightarrow \mathbb{B}_{\text {cris }, J}^{\nabla} \longrightarrow \mathbb{B}_{\text {cris }, J} \otimes_{\mathcal{O}_{\mathcal{U}_{J}}} \Omega_{\mathcal{U}_{J} / \mathcal{O}_{K}}^{*} \longrightarrow 0
$$

The exactness is proven as in proposition 2.37 using the first description given in lemma 3.21 . These complexes are compatible if we vary $J$. In particular we get a double complex $\mathbb{B}_{\text {cris, }} \otimes_{\mathcal{O}_{\mathcal{u}}}$ $\Omega_{\mathcal{U}_{\bullet} / \mathcal{O}_{K}}^{*}$ which is equivalent to the simple complex $\mathbb{B}_{\text {cris, } \bullet}^{\nabla}$. Since the $\mathcal{U}_{i}$ 's cover $X$ the sequence

$$
0 \longrightarrow \mathcal{O}_{\mathfrak{X}_{\bar{K}}} / p \mathcal{O}_{\mathfrak{X}_{\bar{K}}} \longrightarrow j_{\bullet, *}\left(\mathcal{O}_{\mathfrak{u} \bullet_{\bar{K}}} / p \mathcal{O}_{\mathfrak{\mathfrak { u } ^ { \bullet }}}\right) \longrightarrow 0
$$

is exact. Using 2.26 we deduce that the sequence

$$
0 \longrightarrow \mathbb{B}_{\text {cris }, \overline{\mathrm{K}}}^{\nabla} \longrightarrow \mathbb{B}_{\text {cris }, \bullet}^{\nabla} \longrightarrow 0
$$

is also exact. Consider the commutative diagram

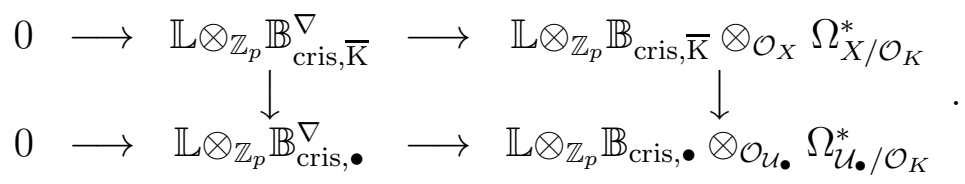

Since the rows are exact and the first column is exact it follows that the complex $\mathbb{L} \otimes_{\mathbb{Z}_{p}} \mathbb{B}_{\text {cris, } \overline{\mathrm{K}}} \otimes_{\mathcal{O}_{X}}$ $\Omega_{X / \mathcal{O}_{K}}^{*}$ is quasi-isomorphic to the double complex $\mathbb{L} \otimes_{\mathbb{Z}_{p}} \mathbb{B}_{\text {cris, } \bullet} \otimes_{\mathcal{O}_{\mathcal{U}_{\bullet}}} \Omega_{\mathcal{U}_{\bullet} / \mathcal{O}_{K}}^{*}$. On the latter the Frobenius maps $F_{J}$ on $\mathcal{U}_{J}$ and Frobenius on $\mathbb{A}_{\text {inf, } J}^{+}$define a morphism of complexes

$$
\Phi_{\bullet, *}: \mathbb{L} \otimes_{\mathbb{Z}_{p}} \mathbb{B}_{\text {cris }, \bullet} \otimes_{\mathcal{O}_{\mathcal{U}_{\bullet}}} \Omega_{\mathcal{U}_{\bullet} / \mathcal{O}_{K}}^{*} \longrightarrow \mathbb{L} \otimes_{\mathbb{Z}_{p}} \mathbb{B}_{\text {cris }, \bullet} \otimes_{\mathcal{O}_{\mathcal{U}_{\bullet}}} \Omega_{\mathcal{U}_{\bullet} / \mathcal{O}_{K}}^{*}
$$

compatible with Frobenius on $\mathbb{L} \otimes_{\mathbb{Z}_{p}} \mathbb{B}_{\text {cris, } \overline{\mathrm{K}}}^{\nabla}$. Let $\mathbb{D}(\mathbb{L})$ be the Frobenius crystal associated to $\mathbb{D}_{\text {cris }}^{\text {ar }}(\mathbb{L})$ (up to isogeny); see the notation following theorem 3.15. By definition of crystal the $\mathcal{O}_{\mathcal{U}_{J}^{\mathrm{PD}}}$-module $\mathbb{D}(\mathbb{L})_{J}:=\left.\mathbb{D}(\mathbb{L})\right|_{\mathcal{U}_{J}^{\mathrm{PD}}}$ together with Frobenius and connection, coincides with the pull-back of $\mathbb{D}_{\text {cris }}^{\text {ar }}(\mathbb{L})$ via the $h$-th projection $\pi_{h}: \mathcal{U}^{J} \rightarrow \mathcal{U}_{h}$. We have

Lemma 3.22. The $\mathcal{O}_{\mathcal{U}_{J}^{\mathrm{PD}}} \widehat{\otimes} B_{\text {cris }}-$ module $\mathbb{D}(\mathbb{L})_{J} \widehat{\otimes} B_{\text {cris }}$ together with Frobenius and connection, coincides with $v_{X, \bar{K}, *}^{\text {cont }}\left(\mathbb{L}_{\mathbb{Z}_{p}} \mathbb{B}_{\text {cris }, J}\right)$ with the Frobenius and connection induced by those on $\mathbb{B}_{\text {cris }, J}$. 
Proof. By definition of crystal $\mathbb{D}(\mathbb{L})_{J}$ coincides with the pull-back of $\mathbb{D}_{\text {cris }}^{\text {ar }}(\mathbb{L})$ via the $h$-th projection $\pi_{h}: \mathcal{U}^{J} \rightarrow \mathcal{U}_{h}$. In particular $\mathcal{O}_{\mathcal{U}_{J}^{\text {PD }}} \cong \pi_{h}^{*}\left(\mathcal{O}_{\mathcal{U}_{h}}\right)\left\langle Y_{i, 1}^{(h)}, \ldots, Y_{i, d}^{(h)}\right\rangle_{i \in J, i \neq h}$ compatibly with Frobenius and connection; see lemma 3.21 for the notation. Due to the second description in 3.21 we get that $v_{X, K, *}^{\text {cont }}\left(\mathbb{B}_{\text {cris }, J}\right) \cong \mathcal{O}_{\mathcal{U}_{J}^{\text {pD }}} \widehat{\otimes} B_{\text {cris }}$ compatibly with Frobenius and connection. Since $\mathbb{L} \otimes_{\mathbb{Z}_{p}} \mathbb{B}_{\text {cris }, J} \cong \mathbb{L} \otimes_{\mathbb{Z}_{p}} j_{J}^{*}\left(\mathbb{B}_{\text {cris }, X}\right) \otimes_{j_{J}^{*}\left(\mathbb{B}_{\text {cris }, X}\right)} \otimes \mathbb{B}_{\text {cris }, J}$ by lemma 3.21 and $\mathbb{L}$ is crystalline, we conclude that $v_{X, \bar{K}, *}^{\text {cont }}$ of the former coincides with $\mathbb{D}_{\text {cris }}^{\text {geo }}(\mathbb{L}) \widehat{\otimes}_{\pi_{h}^{*}\left(\mathcal{O}_{h}\right)} \mathcal{O}_{\mathcal{U}_{J}^{\text {PD }}}$ compatibly with Frobenius and connection. This coincides with $\mathbb{D}(\mathbb{L})_{J} \widehat{\otimes} B_{\text {cris }}$ and the claim follows.

By adjunction we get a morphism of complexes

$$
v_{X, \bar{K}}^{*}\left(\mathbb{D}(\mathbb{L}) \bullet \otimes \Omega_{\mathcal{U}_{\bullet} / \mathcal{O}_{K}}^{*} \widehat{\otimes} B_{\text {cris }}\right) \longrightarrow \mathbb{L} \otimes_{\mathbb{Z}_{p}} \mathbb{B}_{\text {cris, }} \otimes_{\mathcal{O}_{\mathcal{U}_{\bullet}}} \Omega_{\mathcal{U}_{\bullet} / \mathcal{O}_{K}}^{*}
$$

which is compatible with the Frobenius morphisms defined on the two complexes. We deduce that the morphisms

$$
\begin{array}{cc}
\mathrm{H}^{i}\left(\mathfrak{X}_{\bar{K}}, \mathbb{L} \otimes_{\mathbb{Z}_{p}} \mathbb{B}_{\text {cris }, \bar{K}}^{\nabla}\right) & \stackrel{\sim}{\longrightarrow} \mathbb{H}\left(\mathfrak{X}_{\bar{K}}, \mathbb{L} \otimes_{\mathbb{Z}_{p}} \mathbb{B}_{\text {cris, }} \otimes_{\mathcal{O}_{\mathcal{U}_{\bullet}}} \Omega_{\mathcal{U}_{\bullet} / \mathcal{O}_{K}}^{*}\right) \\
\mathrm{H}_{\mathrm{dR}}^{i}\left(X^{\mathrm{et}}, \mathbb{D}_{\text {cris }}^{\mathrm{ar}}(\mathbb{L}) \otimes_{\mathcal{O}_{X}} \Omega_{X / \mathcal{O}_{K}}^{*} \widehat{\otimes} B_{\text {cris }}\right) & \stackrel{\sim}{\longrightarrow} \mathrm{H}_{\mathrm{dR}}^{i}\left(X^{\mathrm{et}}, \mathbb{D}(\mathbb{L}) \bullet \otimes_{\mathcal{O}_{\mathcal{U}_{\bullet}}} \Omega_{\mathcal{U}_{\bullet} / \mathcal{O}_{K}}^{*} \widehat{\otimes} B_{\text {cris }}\right)
\end{array}
$$

are compatible with the Frobenius morphisms defined on each cohomology group. By construction the group $\mathrm{H}_{\mathrm{dR}}^{i}\left(X^{\mathrm{et}}, \mathbb{D}_{\text {cris }}^{\text {geo }}(\mathbb{L})\right)$ is $\mathrm{H}_{\mathrm{dR}}^{i}\left(X^{\mathrm{et}}, \mathbb{D}_{\text {cris }}^{\text {ar }}(\mathbb{L}) \otimes \Omega_{X, \mathcal{O}_{K}}^{*} \widehat{\otimes} B_{\text {cris }}\right)$. The compatibility with Frobenius follows.

\subsection{The comparison isomorphism in the proper case}

Let us now assume that our formal scheme $X \longrightarrow \operatorname{Spf}\left(\mathcal{O}_{K}\right)$ is the formal completion along the special fiber of a proper and smooth scheme $X^{\text {alg }} \longrightarrow \operatorname{Spec}\left(\mathcal{O}_{K}\right)$. We have a morphism of sites $\mu_{M}: X_{M}^{\text {alg,et }} \rightarrow X_{M}^{\text {et }}$ associating $U \mapsto \widehat{U}_{K}$ where $\widehat{U}$ is the $p$-adic completion of $U$. Given a sheaf $\mathbb{L} \in \operatorname{Sh}\left(X_{M}^{\text {alg,et }}\right)$ we write $\mathbb{L}$ for $\mu_{M}^{*}(\mathbb{L})$. Define $\operatorname{Sh}\left(X_{M}^{\text {alg,et }}\right)_{\mathbb{Q}_{p}}^{\text {cris }}$ to be the category of $\mathbb{Q}_{p}$-adic sheaves on $X_{M}^{\text {alg }}$ whose images via $\mu_{M}^{*}$ lie in $\operatorname{Sh}\left(X_{M}^{\text {et }}\right)_{\mathbb{Q}_{p}}^{\text {cris }}$. Given an object $\mathbb{L}$ in $\operatorname{Sh}\left(X_{M}^{\text {alg,et }}\right)_{\mathbb{Q}_{p}}^{\text {cris }}$, we abuse notation and write $\mathbb{D}_{\text {cris }}^{\text {ar }}(\mathbb{L})$ for the filtered locally free $\mathcal{O}_{X_{M_{0}}}$-module with integrable connection on $X_{M_{0}}^{\text {alg }}$ associated by rigid analytic GAGA to the isocrystal $\mathbb{D}_{\text {cris }}^{\text {ar }}\left(\mu_{M}^{*}(\mathbb{L})\right)$. We also identify, for $i \geq 0$ the de Rham cohomology groups $\mathrm{H}_{\mathrm{dR}}^{i}\left(X_{M_{0}}^{\text {alg,Zar }}, \mathbb{D}_{\text {cris }}^{\text {ar }}(\mathbb{L})\right)$ with the rigid cohomology of the $F$-isocrystal $\mathbb{D}_{\text {cris }}^{\text {ar }}\left(\mu_{M}^{*}(\mathbb{L})\right)$ to get a Frobenius structure. Then we have.

Theorem 3.23. There is an isomorphism of $\delta$-functors from $\operatorname{Sh}\left(X_{M}^{\text {alg,et }}\right)_{\mathbb{Q}_{p}}^{\text {cris }}$ to the category of filtered $B_{\text {cris }}-$ modules endowed with $G_{M}$-action and Frobenius:

$$
\mathrm{H}^{i}\left(X_{\bar{K}}^{\text {alg,et }}, \mathbb{L}\right) \otimes_{\mathbb{Z}_{p}} B_{\text {cris }} \cong \mathrm{H}_{\text {cris }}^{i}\left(X / M_{0}, \mathbb{D}_{\text {cris }}^{\text {ar }}(\mathbb{L})\right) \otimes_{M_{0}} B_{\text {cris }}
$$

Here $\mathrm{H}_{\text {cris }}^{i}\left(X / M_{0}, \mathbb{D}_{\text {cris }}^{\text {ar }}(\mathbb{L})\right)$ is the crystalline cohomology of the filtered $F$-isocrystal $\mathbb{D}_{\text {cris }}^{\text {ar }}(\mathbb{L})$ in the sense of [B3]. It is endowed with Frobenius. As it coincides with $\mathrm{H}_{\mathrm{dR}}^{i}\left(X_{M_{0}}^{\mathrm{rig}}, \mathbb{D}_{\text {cris }}^{\mathrm{ar}}(\mathbb{L})\right) \cong$ $\mathrm{H}_{\mathrm{dR}}^{i}\left(X_{M_{0}}^{\mathrm{alg}}, \mathbb{D}_{\mathrm{cris}}^{\mathrm{ar}}(\mathbb{L})\right)$, it is endowed also with the Hodge filtration. The theorem implies the following corollary: 
Corollary 3.24. Let $\mathbb{L}$ be a crystalline étale sheaf on $X_{M}^{\mathrm{alg}}$. Then $\mathrm{H}^{i}\left(X_{\bar{K}}^{\mathrm{alg}, \mathrm{et}}, \mathbb{L}\right)$ is a crystalline representation of $G_{M}$ and $\mathrm{H}_{\mathrm{dR}}^{i}\left(X_{M_{0}}^{\text {alg }}, \mathbb{D}_{\text {cris }}^{\text {ar }}(\mathbb{L})\right)$ is, as filtered $M_{0}$-vector space endowed with Frobenius, the classical $D_{\text {cris }}\left(\mathrm{H}^{i}\left(X_{\bar{K}}^{\text {alg,et }}, \mathbb{L}\right)\right)$ associated to the $G_{M}$-representation $\mathrm{H}^{i}\left(X_{\bar{K}}^{\text {alg,et }}, \mathbb{L}\right)$.

We start with the following:

Proposition 3.25. The natural map

$$
\mathrm{H}_{\text {cris }}^{i}\left(X / M_{0}, \mathbb{D}_{\text {cris }}^{\text {ar }}(\mathbb{L})\right) \otimes_{M_{0}} B_{\text {cris }} \longrightarrow \mathrm{H}^{i}\left(\mathfrak{X}_{\bar{K}}, \mathbb{L} \otimes \mathbb{B}_{\text {cris }, \bar{K}}^{\nabla}\right)
$$

deduced from the isomorphism $\mathbb{D}_{\text {cris }}^{\text {ar }}(\mathbb{L}) \widehat{\otimes}_{M_{0}} B_{\text {cris }} \cong \mathbb{D}_{\text {cris }}^{\text {geo }}(\mathbb{L})$ of 3.9 and from the isomorphism in 3.15, is an isomorphism of $\delta$-functors from $S h\left(X_{M}^{\text {et }}\right)_{\mathbb{Q}_{p}}^{\text {cris }}$ to the category of $B_{\text {cris }}-$ modules endowed with filtrations and Galois action of $G_{M}$.

Proof. Recall that we have an isomorphism $\mathbb{D}_{\text {cris }}^{\text {ar }}(\mathbb{L}) \widehat{\otimes}_{M_{0}} B_{\text {cris }} \cong \mathbb{D}_{\text {cris }}^{\text {geo }}(\mathbb{L})$ as filtered as $\mathcal{O}_{X} \widehat{\otimes}_{\mathcal{O}_{M_{0}}} B_{\text {cris }}-$ modules endowed with filtrations and Galois action of $G_{M}$. Due to 3.15 it suffices to show that the natural map

$$
\gamma_{\mathbb{L}}^{i}: H_{\text {cris }}^{i}\left(X / M_{0}, \mathbb{D}_{\text {cris }}^{\text {ar }}(\mathbb{L})\right) \otimes_{M_{0}} B_{\text {cris }} \longrightarrow \mathrm{H}_{\mathrm{dR}}^{i}\left(X^{\mathrm{et}}, \mathbb{D}_{\text {cris }}^{\text {geo }}(\mathbb{L})\right)
$$

is an isomorphism of $B_{\text {cris }}-$ modules endowed with filtrations and Galois action of $G_{M}$. It is clear that $\gamma_{\mathbb{L}}^{i}$ is $B_{\text {cris }}$-linear and that it is compatible with $G_{M}$-action. Let $\mathbb{D}(\mathbb{L})$ be a Frobenius crystal on $X$ whose generic fiber is $\mathbb{D}_{\text {cris }}^{\text {ar }}(\mathbb{L})$. Then $\mathrm{H}_{\text {cris }}^{i}\left(X / M_{0}, \mathbb{D}_{\text {cris }}^{\text {ar }}(\mathbb{L})\right) \cong \mathrm{H}_{\text {cris }}^{i}\left(X_{k} / \mathcal{O}_{M_{0}}, \mathbb{D}(\mathbb{L})\right)\left[p^{-1}\right]$ as $M_{0}$-vector spaces with filtration and Frobenius. In particular using the definition of the filtration and of Frobenius on $\mathrm{H}_{\mathrm{dR}}^{i}\left(X^{\mathrm{et}}, \mathbb{D}_{\text {cris }}^{\text {geo }}(\mathbb{L})\right)$ we deduce that $\gamma_{\mathbb{L}}^{i}$ is compatible also with the filtrations and with Frobenius.

Since $H_{\mathrm{dR}}^{i}\left(X, \mathbb{D}(\mathbb{L}) \otimes \Omega_{X / \mathcal{O}_{K}}^{\bullet} \cong \mathbb{H}^{i}\left(X^{\mathrm{et}}, \mathbb{D}(\mathbb{L}) \otimes \Omega_{X / \mathcal{O}_{K}}^{\bullet}\right)\right.$ is a finite $\mathcal{O}_{K}$-module and $A_{\text {cris }}$ is $p$-torsion free the natural map

$$
\rho_{\mathbb{L}}^{i}: \mathrm{H}_{\mathrm{dR}}^{i}\left(X, \mathbb{D}(\mathbb{L}) \otimes \Omega_{X / \mathcal{O}_{K}}^{\bullet}\right) \otimes A_{\text {cris }} \longrightarrow \mathbb{H}^{i}\left(X^{\mathrm{et}}, \mathbb{D}(\mathbb{L}) \otimes \Omega_{X / \mathcal{O}_{K}}^{\bullet} \widehat{\otimes} A_{\text {cris }}\right)
$$

is an isomorphism. Inverting $t$ and using the fact that $\mathbb{H}^{i}\left(X^{\mathrm{et}},{ }_{-}\right)$commutes with direct limits since $X$ is noetherian gives the morphism $\gamma_{\mathbb{L}}^{i}$ which is an isomorphism. We are left to prove that $\rho_{\mathbb{L}}^{i}$ is strict after inverting $p$ i.e., that it induces an isomorphism on the various steps of the filtrations.

We first treat the case that $i=2 d$ where $d$ is be the relative dimension of $X$ over $\mathcal{O}_{K}$ and $\mathbb{L}=\mathbb{Z}_{p}$ is the constant sheaf. Then the natural map

$$
\mathrm{H}^{d}\left(X_{K}, \Omega_{X_{K} / K}^{d}\right) \longrightarrow \mathrm{H}_{\mathrm{dR}}^{2 d}\left(X, \Omega_{X_{K} / K}^{\bullet}\right)
$$

is an isomorphism with filtration $\mathrm{Fil}^{n}=$ everything for $n \leq d$ and $\mathrm{Fil}^{n}=0$ for $n>d$. Via the trace map we have an identification $\mathrm{H}^{2 d}\left(X, \Omega_{X_{K} / K}^{d}\right) \cong K(-d)$, where $K(-d)$ is $K$ as a $K$-vector space with $\mathrm{Fil}^{n} K(-d)=K$ for $n \leq d$ and 0 for $n>d$.

Since $\rho_{\mathbb{Z}_{p}}^{2 d}$ is an isomorphism it follows that the map

$$
\mathrm{H}^{d}\left(X, \Omega_{X / \mathcal{O}_{K}}^{d}\right) \otimes A_{\text {cris }}\left[p^{-1}\right] \longrightarrow \mathbb{H}^{2 d}\left(X^{\mathrm{et}}, \Omega_{X / \mathcal{O}_{K}}^{\bullet} \widehat{\otimes} A_{\text {cris }}\right)\left[p^{-1}\right]
$$


is an isomorphism. The quotient of $\Omega_{X / \mathcal{O}_{K}}^{d} \widehat{\otimes} \mathrm{Fil}^{n-d} A_{\text {cris }}[-d] \rightarrow \Omega_{X / \mathcal{O}_{K}}^{\bullet} \widehat{\otimes} \mathrm{Fil}^{n-\bullet} A_{\text {cris }}$ is a complex of quasi-coherent sheaves with $\leq d-1$ terms so that it has trivial cohomology groups $\mathrm{H}^{i}$ for $i \geq 2 d$. In particular $\mathrm{Fil}^{n}$ on $\mathbb{H}^{d}\left(X^{\mathrm{et}}, \Omega_{X / \mathcal{O}_{K}} \widehat{\otimes} A_{\text {cris }}\right)$ is the image of $\mathbb{H}^{d}\left(X^{\mathrm{et}}, \Omega_{X / \mathcal{O}_{K}}^{d} \widehat{\otimes} \mathrm{Fil}^{n-d} A_{\text {cris }}\right)$. This coincides with $\mathrm{H}^{d}\left(X, \Omega_{X, \mathcal{O}_{K}}^{d}\right) \otimes \mathrm{Fil}^{n-d} A_{\text {cris }}$ showing that $\rho_{\mathbb{Z}_{p}}^{2 d}$ is an isomorphism of filtered $A_{\text {cris }^{-}}$ modules.

In the general case recall that $\mathbb{D}\left(\mathbb{L}^{\vee}\right) \cong \mathbb{D}(\mathbb{L})^{\vee}$ as filtered isocrystals by 3.12 i.e., the pairing provides on filtrations morphisms $\mathrm{Fil}^{n} \mathbb{D}(\mathbb{L}) \times \mathrm{Fil}^{h} \mathbb{D}\left(\mathbb{L}^{\vee}\right) \rightarrow \mathrm{Fil}^{n+h} \mathcal{O}_{X}$. This and the fact that $\rho_{\mathbb{Z}_{p}}^{2 d}$ is an isomorphism of filtered modules provides with pairings of filtered modules

$$
\begin{aligned}
& \begin{aligned}
\mathrm{H}_{\mathrm{dR}}^{i}\left(X, \mathbb{D}(\mathbb{L}) \otimes \Omega_{X / \mathcal{O}_{K}}^{\bullet}\right) \otimes \mathrm{H}_{\mathrm{dR}}^{2 d-i}\left(X, \mathbb{D}\left(\mathbb{L}^{\vee}\right) \otimes \Omega_{X / \mathcal{O}_{K}}^{\bullet}\right) \otimes A_{\text {cris }} & \longrightarrow \quad A_{\text {cris }}(-d) \\
\left.\rho_{\mathbb{L}}^{i} \otimes \rho_{\mathbb{L}^{\vee}}^{2 d-i}\right\rfloor & \|
\end{aligned} \\
& \mathbb{H}^{i}\left(X^{\mathrm{et}}, \mathbb{D}(\mathbb{L}) \otimes \Omega_{X / \mathcal{O}_{K}}^{\bullet} \widehat{\otimes} A_{\text {cris }}\right) \otimes \mathbb{H}^{2 d-i}\left(X^{\mathrm{et}}, \mathbb{D}\left(\mathbb{L}^{\vee}\right) \otimes \Omega_{X / \mathcal{O}_{K}} \widehat{\otimes} A_{\text {cris }}\right) \longrightarrow A_{\text {cris }}(-d) \text {. }
\end{aligned}
$$

By [S, Prop. 2.5.3] Poincaré duality induces an isomorphism of filtered $K$-vector spaces

$$
\mu_{\mathbb{L}}^{i}: \mathrm{H}_{\mathrm{dR}}^{i}\left(X, \mathbb{D}(\mathbb{L}) \otimes \Omega_{X / \mathcal{O}_{K}}^{\bullet}\right)\left[p^{-1}\right] \longrightarrow \operatorname{Hom}\left(\mathrm{H}_{\mathrm{dR}}^{2 d-i}\left(X, \mathbb{D}(\mathbb{L}) \otimes \Omega_{X / \mathcal{O}_{K}}^{\bullet}\right), K(-d)\right) .
$$

Recall that $\mathrm{Fil}^{n} \operatorname{Hom}\left(\mathrm{H}_{\mathrm{dR}}^{2 d-i}\left(X, \mathbb{D}(\mathbb{L}) \otimes \Omega_{X / \mathcal{O}_{K}}^{\bullet}\right), K(-d)\right)$ consists of the $f: \mathrm{H}_{\mathrm{dR}}^{2 d-i}(X, \mathbb{D}(\mathbb{L}) \otimes$ $\left.\Omega_{X / \mathcal{O}_{K}}\right) \rightarrow K(-d)$ such that $f\left(\mathrm{Fil}^{h}\right) \subset \mathrm{Fil}^{h+n}$ for every $h$. In particular the pairings displayed above give then morphisms of filtered $A_{\text {cris }}$-modules:

$$
\begin{gathered}
\mathrm{H}_{\mathrm{dR}}^{i}\left(X, \mathbb{D}(\mathbb{L}) \otimes \Omega_{X / \mathcal{O}_{K}}^{\bullet} \otimes A_{\text {cris }}\left[p^{-1}\right] \longrightarrow \mathbb{H}^{i}\left(X^{\mathrm{et}}, \mathbb{D}(\mathbb{L}) \otimes \Omega_{X / \mathcal{O}_{K}}^{\bullet} \widehat{\otimes} A_{\text {cris }}\right)\left[p^{-1}\right] \longrightarrow\right. \\
\longrightarrow \operatorname{Hom}_{A_{\text {cris }}}\left(\mathbb{H}^{2 d-i}\left(\left(X^{\mathrm{et}}, \mathbb{D}\left(\mathbb{L}^{\vee}\right) \otimes \Omega_{X / \mathcal{O}_{K}}^{\bullet} \widehat{\otimes} A_{\text {cris }}\right), A_{\text {cris }}(-d)\right)\left[p^{-1}\right] \longrightarrow\right. \\
\longrightarrow \operatorname{Hom}_{A_{\text {cris }}}\left(\mathrm{H}_{\mathrm{dR}}^{2 d-i}\left(X, \mathbb{D}\left(\mathbb{L}^{\vee}\right) \otimes \Omega_{X / \mathcal{O}_{K}}^{\bullet}\right) \otimes A_{\text {cris }}, A_{\text {cris }}(-d)\right)\left[p^{-1}\right]
\end{gathered}
$$

such that the composite is $\mu_{\mathbb{L}}^{i} \otimes A_{\text {cris }}$. In particular it is an isomorphism of filtered $A_{\text {cris }}$-modules. This implies that the first map, which is $\rho_{\mathbb{L}}^{i}\left[p^{-1}\right]$, is an isomorphism as filtered $A_{\text {cris }}\left[p^{-1}\right]$-modules as claimed.

Let us now assume that $\mathbb{L}$ is a crystalline étale sheaf on $X_{M}$. For $i \in \mathbb{Z}$ write $V_{i}:=$ $\mathrm{H}^{i}\left(X_{\bar{K}}^{\mathrm{et}}, \mathbb{L}\right) \otimes_{\mathbb{Z}_{p}} \mathbb{Q}_{p}$ and $D_{i}:=\mathrm{H}_{\mathrm{dR}}^{i}\left(X_{K}, \mathbb{D}_{\text {cris }}^{\text {ar }}(\mathbb{L})\right)$. Then $V_{i}$ is a finite dimensional $p$-adic representation of $G_{M}$ for every $i \in \mathbb{Z}$ and $V_{i}=0$ unless $0 \leq i \leq 2 d$, where $d$ is the dimension of $X_{K}$. Similarly, $D_{i}$ is a finite dimensional filtered $\varphi$-module over $M_{0}$ for all $i \in \mathbb{Z}$ and $D_{i}=0$ unless $0 \leq i \leq 2 d$.

Corollary 3.26. We have a canonical commutative diagram with exact rows, referred to as the diagram $\left(*_{\mathbb{L}}\right)$, of topological $\mathbb{Q}_{p}$-vector spaces with continuous $G_{M}$-action:

$$
\begin{aligned}
& \cdots \longrightarrow V_{i} \stackrel{\alpha_{i}}{\longrightarrow} \operatorname{Fil}^{0}\left(D_{i} \otimes_{M_{0}} B_{\text {cris }}\right) \stackrel{1-\varphi}{\longrightarrow} D_{i} \otimes_{M_{0}} B_{\text {cris }} \stackrel{\epsilon_{i}}{\longrightarrow} \quad V_{i+1} \\
& \downarrow \beta_{i} \quad \downarrow \gamma_{i} \quad \| \quad \downarrow \beta_{i+1} \\
& \cdots \longrightarrow T_{i} \stackrel{\omega_{i}}{\longrightarrow} \quad D_{i} \otimes_{M_{0}} B_{\text {cris }} \quad \stackrel{1-\varphi}{\longrightarrow} D_{i} \otimes_{M_{0}} B_{\text {cris }} \longrightarrow\left(D_{i+1} \otimes_{M_{0}} B_{\text {cris }}\right)^{\varphi=1}
\end{aligned}
$$


Proof. Consider the diagram attached to $\mathbb{L}$ tensoring (2) with $\mathbb{L}$ and taking the long exact sequence in cohomology. We get a commutative diagram of $\mathbb{Q}_{p}$-modules endowed with continuous action of $G_{M}$ whose rows are exact:

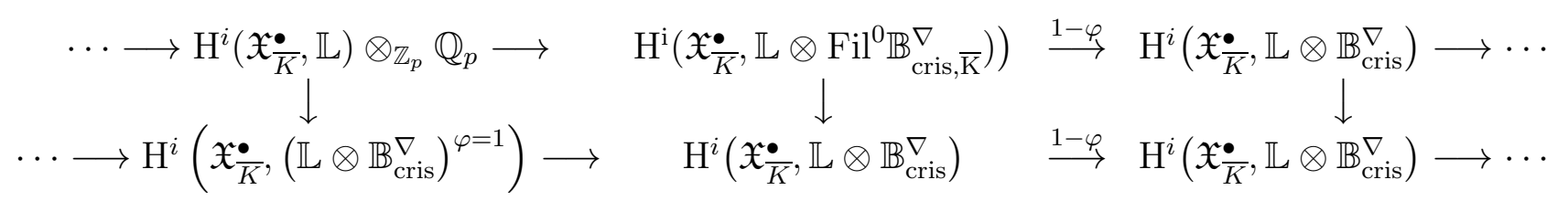

For every $i \in \mathbb{Z}$ we have canonical isomorphisms $\mathrm{H}^{i}\left(\mathfrak{X}_{\bar{K}}, \mathbb{L}\right)[1 / p] \cong V_{i}$ as $G_{M}$-modules by [AI, Prop. 4.9]. By theorem 3.15 and proposition 3.25 we have canonical isomorphisms as

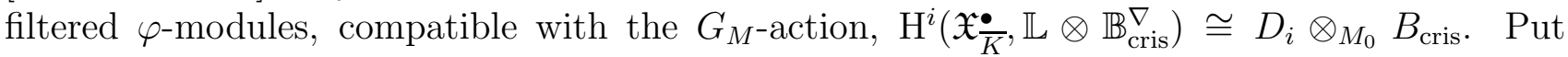
$T_{i}:=\mathrm{H}^{i}\left(\mathfrak{X}_{\bar{K}}^{\bullet},\left(\mathbb{L} \otimes \mathbb{B}_{\text {cris }}^{\nabla}\right)^{\varphi=1}\right)$. Furthermore the image of the map $g_{i}$ from $\left.\mathrm{H}^{\mathrm{i}}\left(\mathfrak{X}_{\bar{K}}^{\bullet}, \mathbb{L} \otimes \mathrm{Fil}^{0} \mathbb{B}_{\text {cris }, \overline{\mathrm{K}}}^{\nabla}\right)\right)$ to $\left.\mathrm{H}^{\mathrm{i}}\left(\mathfrak{X}_{\bar{K}}^{\bullet}, \mathbb{L} \otimes \mathbb{B}_{\text {cris }, \overline{\mathrm{K}}}^{\nabla}\right)\right)$ is $\mathrm{Fil}^{0}\left(\mathrm{H}^{\mathrm{i}}\left(\mathfrak{X}_{\bar{K}}^{\bullet}, \mathbb{L} \otimes \mathrm{Fil}^{0} \mathbb{B}_{\text {cris }, \overline{\mathrm{K}}}^{\nabla}\right)\right)$. To prove the claim it suffices to show that $g_{i}$ is injective. It follows from the above diagram of long exact sequences that the kernel of $g_{i}$ is in the image of $V_{i}$. In particular it is a finite dimensional $\mathbb{Q}_{p}$-vector space. Since $\operatorname{Ker}\left(g_{i}\right)$ is a $B_{\text {cris }}^{+}$-module and $B_{\text {cris }}^{+}$is an algebra over the maximal unramified extension $K^{\text {un }}$ of $K$, then $\operatorname{Ker}\left(g_{i}\right)$ is a $K^{\text {un }}$-vector space. Since $\operatorname{Ker}\left(g_{i}\right)$ is a finite dimensional $\mathbb{Q}_{p}$-vector space we conclude that it must be 0 which proves the claim.

As in corollary 3.26 take $\mathbb{L}$ to be a crystalline sheaf. Then its $\mathbb{Z}_{p}$-dual $\mathbb{L}^{\vee}$ is also a crystalline sheaf on $X_{K}$ and $\mathbb{D}_{\text {cris }}^{\text {ar }}\left(\mathbb{L}^{\vee}\right) \cong \mathbb{D}_{\text {cris }}^{\text {ar }}(\mathbb{L})^{\vee}:=\operatorname{Hom}_{\text {Isoc }(\mathrm{X})}\left(\mathbb{D}_{\text {cris }}^{\text {ar }}(\mathbb{L}), \mathcal{O}_{X_{K}}\right)$ where the isomorphism is as filtered $F$-isocrystals on $X$ (or $\left.X_{\mathbb{F}}\right)$. Let us denote for every $i \in \mathbb{Z}, V_{i}^{*}:=\mathrm{H}^{i}\left(X_{K}^{\mathrm{et}}, \mathbb{L}^{\vee}\right)[1 / p]$ and by $D_{i}^{*}:=\mathrm{H}_{\mathrm{dR}}^{i}\left(X_{K}, \mathbb{D}_{\text {cris }}^{\text {ar }}\left(\mathbb{L}^{\vee}\right)\right)$ the $G_{M}$-representations, respectively the filtered $\varphi$-modules attached to $\mathbb{L}^{\vee}$. By Poincaré duality for étale cohomology and de Rham cohomology respectively we have canonical isomorphisms as $G_{M^{-}}$-representations (respectively as filtered $\varphi$-modules) $V_{i}^{*} \cong$ $V_{2 d-i}^{\vee}$ (respectively $D_{i}^{*} \cong D_{2 d-i}^{\vee}$.) Let us remark that we have a canonical diagram with exact rows attached to $\mathbb{L}^{\vee}$, denoted $\left(*_{\mathbb{L}} \vee\right)$, which involves $V_{i}^{*}, D_{i}^{*}$ and in which the maps are denoted $\alpha_{i}^{*}, \beta_{i}^{*}, \gamma_{i}^{*}, \ldots$

Suppose $\mathbb{L}$ is a crystalline sheaf on $X_{M}^{\mathrm{et}}$ and assume all the notations above. The idea of the proof of Theorem 3.23 is very simple. We prove by induction on $j \geq 0$ that $D_{2 d-j}$ is an admissible filtered $\varphi$-module and that $V_{\text {cris }}\left(D_{2 d-j}\right)=V_{2 d-j}$. Granting this it follows that $V_{2 d-j}$ is a crystalline representation of $G_{M}$ and that $D_{\text {cris }}\left(V_{2 d-j}\right) \cong D_{2 d-j}$ and so we are done.

Let us first recall a criterion of admissibility from $[\mathrm{CF}]$. In our setting $M_{0}$ is a finite unramified extension of $\mathbb{Q}_{p}$ and $D$ is a finite dimensional filtered $\varphi$-module over $M_{0}$. Let

$$
\delta(D):\left(D \otimes_{M_{0}} B_{\text {cris }}\right)^{\varphi=1} \longrightarrow \frac{D \otimes_{M_{0}} B_{\text {cris }}}{\operatorname{Fil}^{0}\left(D \otimes_{M_{0}} B_{\text {cris }}\right)}
$$

be the natural map. Put $V_{\text {cris }}(D):=\operatorname{Ker}\left(\delta_{D}\right)$

Proposition 3.27 ([CF]). The filtered $\varphi$-module $D$ over $M_{0}$ is admissible if and only if (a) $V_{\text {cris }}(D)$ is a finite dimensional $\mathbb{Q}_{p}$-vector space and (b) $\delta(D)$ is surjective.

Moreover, if $V=V_{\text {cris }}(D)$ is finite dimensional then it is a crystalline representation of $G_{M}$ and $D_{\text {cris }}(V) \subseteq D$. This inclusion is an equality if and only if $D$ is admissible. 
Proof. Let us first remark that we are in the situation of [CF], i.e. the natural map

$$
\frac{D \otimes_{M_{0}} B_{\text {cris }}}{\operatorname{Fil}^{0}\left(D \otimes_{M_{0}} B_{\text {cris }}\right)} \longrightarrow \frac{D \otimes_{M_{0}} B_{\mathrm{dR}}}{\operatorname{Fil}^{0}\left(D \otimes_{M_{0}} B_{\mathrm{dR}}\right)}
$$

is an isomorphism for every finite dimensional filtered module $D$ over $M_{0}$ due to the fact that the natural inclusion $B_{\text {cris }} \longrightarrow B_{\mathrm{dR}}$ of filtered rings induces isomorphisms on the graded quotients.

It is proven in $\left[\mathrm{CF}\right.$, Prop. 4.5] that the $\mathbb{Q}_{p}$-vector space $V_{\text {cris }}(D)$ is finite dimensional if and only if for every sub-object $D^{\prime} \subset D$ we have $t_{H}\left(D^{\prime}\right) \leq t_{N}\left(D^{\prime}\right)$. Moreover, it also shown in loc. cit. that in this case $V_{\text {cris }}(D)$ is a crystalline representation of $G_{M}$ whose associated filtered $\varphi$-module is contained in $D$. It coincides with $D$ if and only if $\operatorname{dim}_{\mathbb{Q}_{p}} V_{\text {cris }}(D)=\operatorname{dim}_{M_{0}} D$.

It follows from the proof of of $\left[\mathrm{CF}\right.$, Prop. 5.7] that, if $V_{\text {cris }}(D)$ is finite dimensional, then $\operatorname{dim}_{\mathbb{Q}_{p}} V_{\text {cris }}(D)=\operatorname{dim}_{M_{0}} D$ if and only if $\delta(D)$ is surjective. The claim follows.

The proof of Theorem 3.23. Let $\mathbb{L}$ be a crystalline sheaf on $X_{K}$ and let us consider the part of the diagram $\left(*_{\mathbb{L}}\right)$ relevant for $j=0$ :

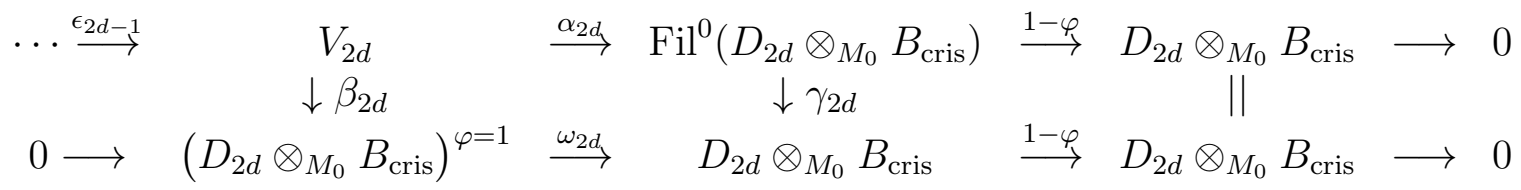

Let us remark that $\delta\left(D_{2 d}\right)$ can be seen as the composition

$$
\left(D_{2 d} \otimes_{M_{0}} B_{\text {cris }}\right)^{\varphi=1} \stackrel{\omega_{2 d}}{\longrightarrow} D_{2 d} \otimes_{M_{0}} B_{\text {cris }} \longrightarrow \operatorname{Coker}\left(\gamma_{2 d}\right)
$$

and also that $\operatorname{Ker}\left(\delta\left(D_{2 d}\right)\right)=\operatorname{Ker}\left((1-\varphi): \mathrm{Fil}^{0}\left(D_{2 d} \otimes_{M_{0}} B_{\text {cris }}\right) \longrightarrow D_{2 d} \otimes_{M_{0}} B_{\text {cris }}\right)$. It follows that $\alpha_{2 d}$ induces a surjective $\mathbb{Q}_{p}$-linear map $V_{2 d} \longrightarrow \operatorname{Ker}\left(\delta\left(D_{2 d}\right)\right)$ and that $\delta\left(D_{2 d}\right)$ is surjective. We deduce from proposition 3.27 that $D_{2 d}$ is admissible and that we have a $\mathbb{Q}_{p}$-linear, surjective homomorphism $V_{2 d} \longrightarrow V_{\text {cris }}\left(D_{2 d}\right)$ which is $G_{M}$-equivariant.

Now we look at the part near $i=0$ of the diagram $\left(*_{\mathbb{L}^{\vee}}\right)$, remarking that $D_{0}^{*} \cong D_{2 d}^{\vee}$ by $[\mathrm{S}$, Prop. 2.5.3] and therefore it is admissible.

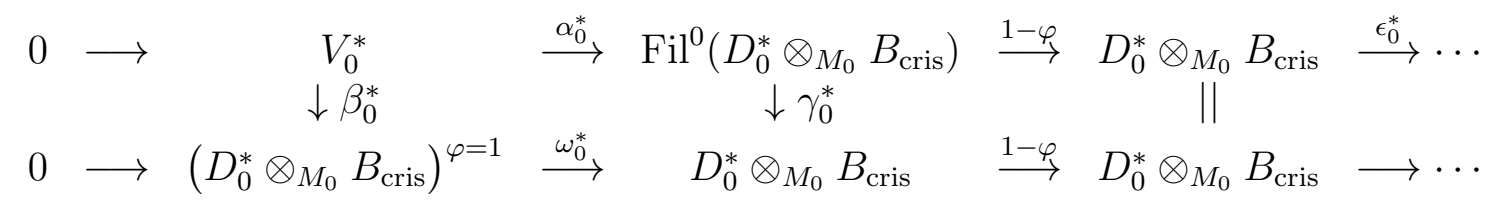

It follows that $V_{0}^{*} \cong \operatorname{Ker}\left(\delta\left(D_{0}^{*}\right)\right)=V_{\text {cris }}\left(D_{0}^{*}\right)$. Therefore we deduce that $\operatorname{dim}_{\mathbb{Q}_{p}}\left(V_{2 d}\right)=\operatorname{dim}_{\mathbb{Q}_{p}}\left(V_{0}^{*}\right)=$ $\operatorname{dim}_{K}\left(D_{0}^{*}\right)=\operatorname{dim}_{K}\left(D_{2 d}\right)=\operatorname{dim}_{\mathbb{Q}_{p}}\left(V_{\text {cris }}\left(D_{2 d}\right)\right)$ and hence $V_{2 d} \cong V_{\text {cris }}\left(D_{2 d}\right)$ and $V_{0}^{*} \cong V_{\text {cris }}\left(D_{0}^{*}\right)$. This proves our statement for $j=0$ for $\mathbb{L}$ and $j=2 d$ for $\mathbb{L}^{\vee}$.

Let us remark at the same time that as $\alpha_{2 d}$ is injective, $\epsilon_{2 d-1}=0$. Since $V_{0}^{*} \cong V_{\text {cris }}\left(D_{0}^{*}\right)$ an easy diagram chase shows that $\epsilon_{0}^{*}=0$ and therefore the map $\alpha_{1}^{*}$ is injective. Since $\gamma_{1}^{*}$ is injective, we can continue with $j=1$ along exactly the same lines as for $j=0$. By induction Theorem 3.23 follows. 


\section{References}

[AB] F. Andreatta, O. Brinon: Acyclicité géométrique de $B_{\text {cris }}$, Submitted. Available at http://www.math.univ-paris13.fr/ brinon.

[AI] F. Andreatta, A. Iovita: Global applications of relative $(\varphi, \Gamma)$-modules, Astérisque 319, Reprśentations $p$-adiques de groupes $p$-adiques I: Représentations galoisiennes et $(\varphi, \Gamma)$ modules (2008), 339-420.

[Err] F. Andreatta, A. Iovita: Erratum to the article "Global applications to relative $\Phi-\Gamma$-modules, I", Astérisque 330, Représentations $p$-adiques de groupes $p$-adiques II: Représentations de $\mathbf{G L}_{2}\left(\mathbb{Q}_{p}\right)$ et $(\varphi, \Gamma)$-modules (2010), 543-554.

[AIS] F. Andreatta, A. Iovita, G. Stevens: A cohomological construction of p-adic families of modular forms, in preparation.

[A] M. Artin: Grothendieck topologies, Notes on a seminar at Harvard University, Spring 1962.

[BO] P. Berthelot, A. Ogus: Notes on crystalline cohomology, Princeton University Press, 1978.

[B1] P. Berthelot: Cohomologie cristalline des schémas de caractéristique $p>0$ LNM 407, Springer, Berlin-Heidelberg-New York, 1974.

[B2] P. Berthelot: Géométrie rigide et cohomologie des variétés algébriques de caractéristique $p$, Introductions aux cohomologies p-adiques (Luminy, 1984). Mém. Soc. Math. France 23 (1986), 7-32.

[B3] P. Berthelot: Cohomologie rigide et cohomologie rigide à supports propres. Premiére partie, Preprint IRMAR 96-03 (1996), 89 pp. Available at http://perso.univ-rennes1.fr/pierre.berthelot.

[BK] S. Bloch, K. Kato: p-adic étale cohomology, Inst. Hautes Études Sci. Publ. Math. 63 (1986), 107-152.

[Bri] O. Brinon: Représentations p-adiques cristallines et de de Rham dans le cas relatif, Mém. Soc. Math. Fr. 112 (2008), 159 pp.

[Bri2] O. Brinon: Représentations cristallines dans le cas d'un corps résiduel imparfait, Ann. Inst. Fourier 56 (2006), 919-999.

[CC] M. Candilera, V. Cristante: Periods and duality of p-adic Barsotti-Tate groups, Ann. Scuola Norm. Sup. Pisa, Cl. Sci. 22 (1995), 545-593.

[Cz] P. Colmez: Périods p-adiques des variétés abélinnes, Math. Ann. 292 (1992), 629-644.

[CF] P. Colmez, J.-M. Fontaine: Construction des représentations p-adiques semi-stables, Invent. Math. 140 (2000), 1-43.

[dJvdP] J. de Jong, M. van der Put: Étale Cohomology of Rigid Analytic Spaces, Doc. Math. 1 (1996), 1-56. 
[F1] G. Faltings: p-Adic Hodge Theory, Journal AMS 1 (1988), 255-299.

[F2] G. Faltings: Crystalline cohomology and p-adic Galois representations, In "Algebraic Analysis, Geomtery and Number Theory" (J.I.Igusa ed.), John Hopkins University Press, Baltimore (1998), 25-80.

[F3] G. Faltings: Almost étale extensions, In "Cohomologies p-adiques et applications arithmétiques," vol. II. P. Berthelot, J.-M. Fontaine, L. Illusie, K. Kato, M. Rapoport eds. Astérisque 279 (2002), 185-270.

[Fo] J.-M. Fontaine: Sur certains types de représentations p-adiques du group de Galois d'un corps local; construction d'un anneau de Barsotti-Tate, Ann. of Math. 115 (1982), 529-577.

[FM] J.-M. Fontaine, W. Messing: p-adic periods and p-adic étale cohomology, In "Current trends in arithmetical algebraic geometry (Arcata, Calif., 1985)", Contemp. Math. 67 (1987), 179-207.

[Fo] J.-M. Fontaine: Le corps des périodes p-adiques. With an appendix by Pierre Colmez. In "Périodes p-adiques (Bures-sur-Yvette, 1988)". Astérisque 223 (1994), 59-111.

[EGAIV] A. Grothendieck, J. Dieudonné: Éléments de Géométrie Algébrique, Inst. Hautes Études Sci. Publ. Math. 24 (1964-1965).

[SGAIV] Théorie des topos et cohomologie étale des schémas. Tome 1: Théorie des topos. Séminaire de Géométrie Algébrique du Bois-Marie 1963-1964 (SGA 4). Dirigé par M. Artin, A. Grothendieck, et J. L. Verdier. Avec la collaboration de N. Bourbaki, P. Deligne et B. Saint-Donat. LNM 269 (1972).

[HK] O. Hyodo, K. Kato: Semi-stable reduction and crystalline cohomology with logarithmic poles, Astérisque 223 (1994), 321-347.

[I] L. Illusie: Cohomologie de de Rham et cohomologie étale p-adique, Astérisque 189-190 (1989).

[LS] B. Le Stum: PhD thesis, Rennes.

[Ka] K. Kato: Semi-stable reduction and p-adic étale cohomology, Asterisque 223 (1994), 269295.

[N] W. Niziol: Crystalline conjecture via K-theory, Ann. Sci. École Norm. Sup. 31, 659-681,

[O] A. Ogus: F-Isocrystals and de Rham cohomology II. Convergent isocrystals. Duke Math. J. 51 (1984), 765-850.

[S] M. Saito: Modules de Hodge polarisables, Publ. Res. Inst. Math. Sci. 24 (1988), 849-995.

[T] T. Tsuji: p-adic étale cohomology and crystalline cohomology in the semi-stable reduction case, Invent. Math. 137 (1999), 233-411. 
[T1] T. Tsuji: Crystalline Sheaves and Filtered Convergent F-Isocrystals on Log Schemes, preprint (2008).

[Va] P. Valabrega: A few theorems on completion of excellent rings, Nagoya Math. J. 61 (1976), $127-133$.

[Y] G. Yamashita: p-Adic Étale Cohomology and Crystalline Cohomology for Open Varieties with Semistable Reduction, preprint (2002).

Fabrizio Andreatta

DIPARTIMENTO DI MATEMATICA "FEDERIGO ENRIQUES",

UNIVERSITÀ STATALE DI MILANO, VIA C. SALDINI 50, 20133, ITALIA

Adrian Iovita

DIPARTIMENTO DI MATEMATICA PURA ED APPLICATA, UNIVERSITÀ DEGLI

STUDI DI PADOVA, VIA TRIESTE 63, PADOVA 35121, ITALIA

and

DEPARTMENT OF MATHEMATICS AND STATISTICS, CONCORDIA UNIVERSITY

1455 DE MAISONNEUVE BLVD., MONTREAL, H3G 1MB CANADA 